\title{
LOW-FLOW CHARACTERISTICS OF OKLAHOMA STREAMS
}

U. S. GEOLOGICAL SURVEY Open-File Report 78-166

\section{1}
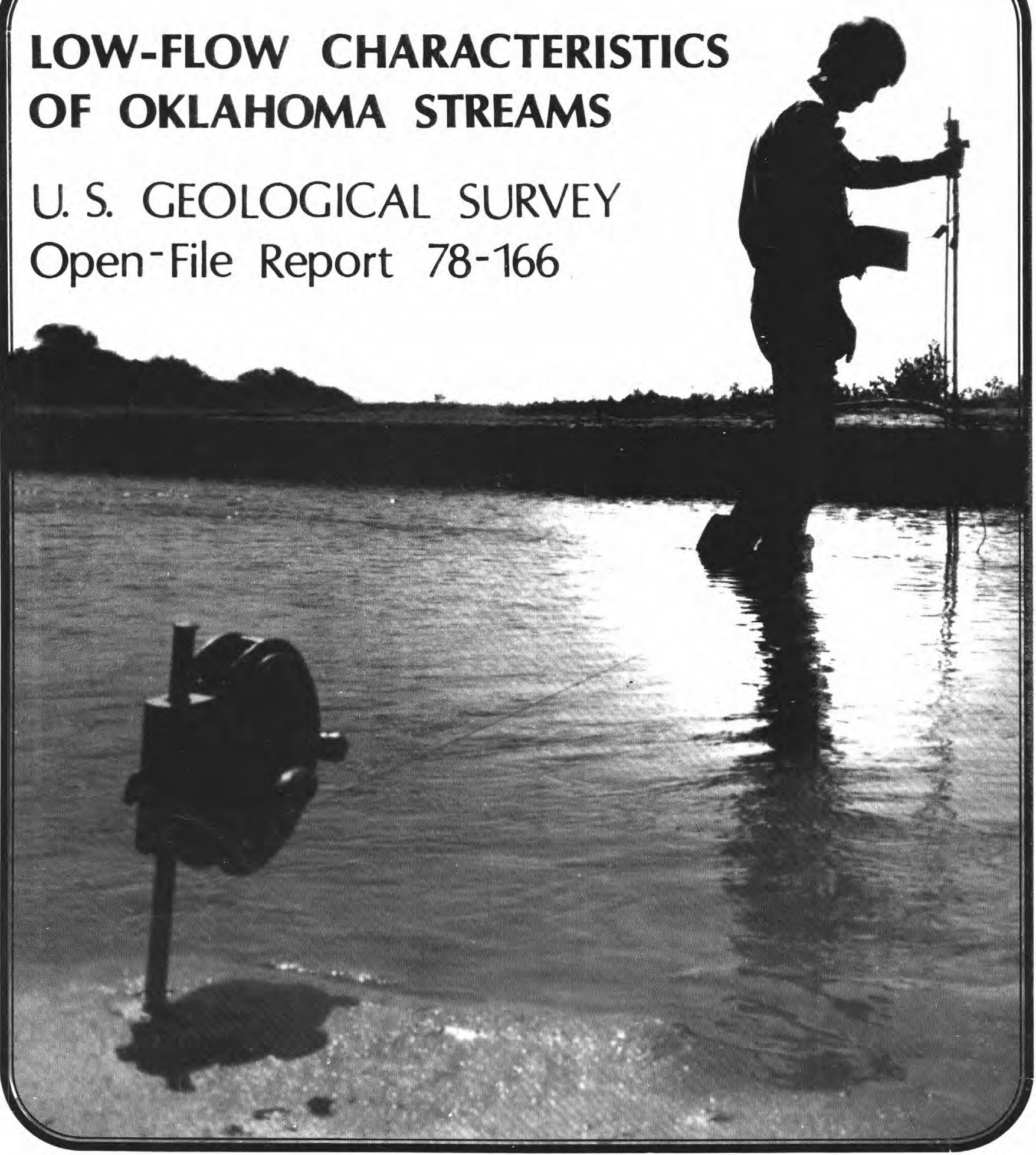

Prepared in cooperation with the OKLAHOMA WATER RESOURCES BOARD

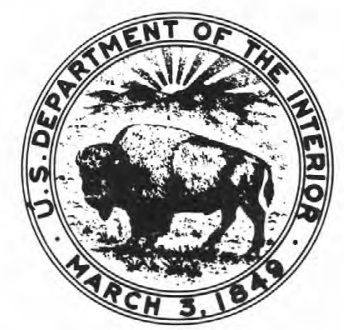





\section{UNITED STATES \\ DEPARTMENT OF THE INTERIOR \\ Geological Survey}

\section{LOW-FLOW CHARACTERISTICS \\ OF OKLAHOMA STREAMS}

By Thomas L. Huntzinger

Open-File Report 78-166

Prepared in cooperation with the

OKLAHOMA WATER RESOURCES BOARD

Oklahoma City, Oklahoma

March, 1978 



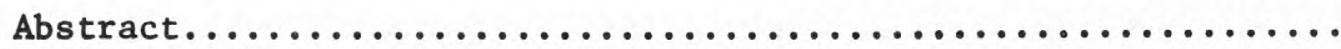

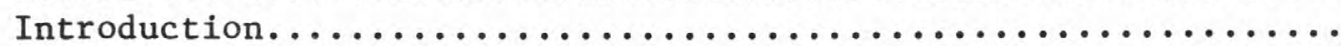

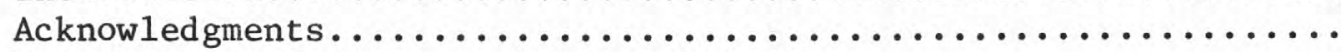

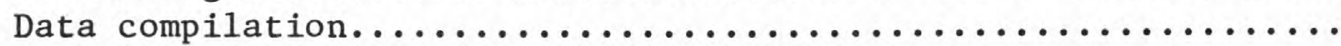

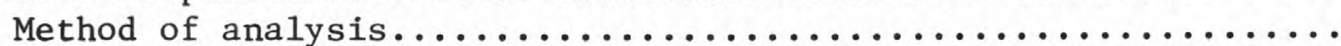

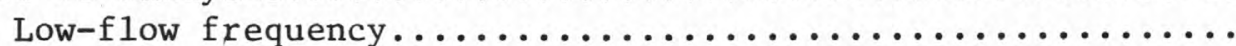

Computation of low-flow characteristics at continuous

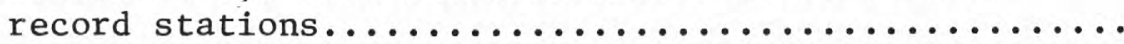

Computation of low-flow characteristics at partial-record

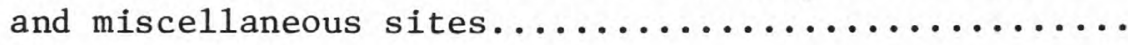

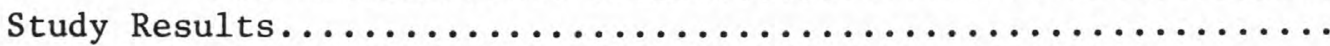

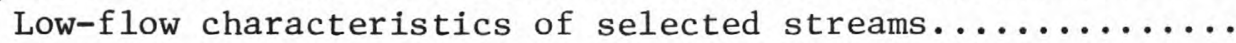

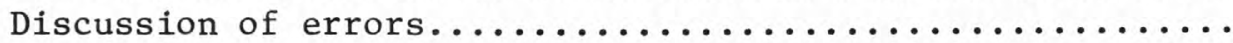

Conclusions and recommendations for further study.........

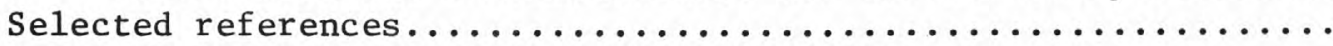
Appendix I, Miscellaneous and low-flow measurement data....... Appendix II, Alphabetical and station number indices...........

\section{ILLUSTRATIONS}

Figure 1.--Map of Oklahoma showing location of streamflow

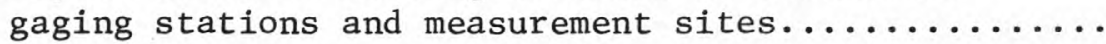

follows

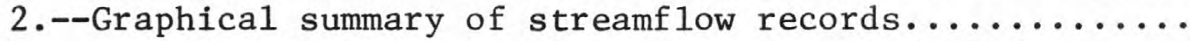

3.--Low-flow frequency curves for selected durations for Clear Boggy Creek near Caney, Oklahoma.........

4.--Map of Oklahoma showing location of index

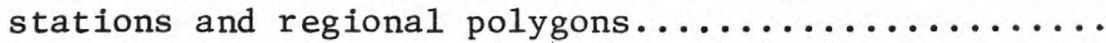

5.--Example of low-flow analysis to obtain low-flow characteristics at a partial record or miscellaneous site

TABLES

Table 1.--Lowest mean discharge and ranking for selected flow durations for year ending March 31 for Clear Boggy

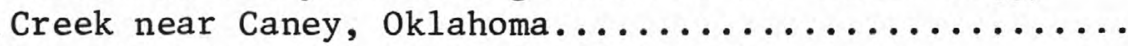

2.--Selected low-flow characteristics of streams

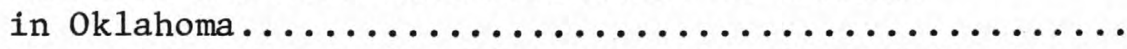

3.--Expected error in low-flow estimates for regional

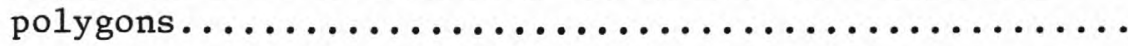


Readers preferring metric units to U.S. customary units are provided the following conversion table.

Multiply U.S. customary unit By To obtain metric unit

foot $(f t)$

0.3048 meter (m)

mile (mi)

1.609 kilometer (km)

square mile $\left(\mathrm{mi}^{2}\right)$

2.590

square kilometer $\left(\mathrm{km}^{2}\right)$

cubic foot per second $\left(\mathrm{ft}^{3} / \mathrm{s}\right)$

0.0283

cubic meter per second $\left(\mathrm{m}^{3} / \mathrm{s}\right)$ 


\title{
LOW-FLOW CHARACTERISTICS OF OKLAHOMA STREAMS
}

by Thomas L. Huntzinger

\begin{abstract}
Analysis of streamflow records in Oklahoma has provided information on the low-flow characteristics of selected streams in Oklahoma. The 2-, 10- and 20-year low-flow frequency for 1-, 7-, 14- and 30-day durations have been determined for 143 continuous record stations, 46 partial record, and 56 miscellaneous sites in Oklahoma. Low-flows of small uncontrolled streams are zero for the State except for northeastern and south-central Oklahoma.
\end{abstract}




\section{INTRODUCTION}

The water resource plays a vital role in the economic welfare of the State of Oklahoma and the personal lives of its people. The dependence upon water for daily functions has created an urgent need for investigating the availability and extent of water resources in 0klahoma. One of many particular water-resource investigations that has been needed is an analysis of low-flow characteristics of streams in the State. Design of storage and treatment facilities and planning for industrial, residential, and recreational development require knowledge about water supply and water quality of streams involved.

Low-flow characteristics have been developed for those streamflow sites with sufficient data for analysis. Many streams have only short-term records or instantaneous discharge measurements made during low-flow periods. The low-flow data for these sites with little or no continuous record has been compiled and related to nearby continuous-record sites to estimate low-flow characteristics.

\section{ACKNOWLEDGMENTS}

The Geological Survey and other organizations have had cooperative agreements for the systematic collection of surface-water records in Oklahoma since 1935. Organizations that have assisted in collecting data with the Geological Survey are: Oklahoma Water Resources Board; Oklahoma City Water Department; Corps of Engineers, U.S. Army; Soil Conservation Service, U.S. Department of Agriculture; Bureau of Reclamation, U.S. Department of the Interior; Grand River Dam Authority; Centra1 Oklahoma Master Conservancy District; Fort Cobb Reservoir Master Conservancy District; Lugert-Altus Irrigation District; Agricultural Research Service, U.S. Department of Agriculture; Cities of Ada, Altus, Lawton, Shawnee, and Tulsa.

\section{DATA COMPILATION}

Data from three types of streamflow sites were used in preparing this report: continuous-record sites, partial-record sites, and miscellaneous sites. A site where long-term daily discharge record is available is termed a continuous streamflow site. Records of this type were separated from the rest and analyzed on an individual basis. Sites where instantaneous low-flow discharge measurements were made on a regular basis specifically to obtain low-flow information are termed partial-record sites. Isolated instantaneous low-flow measurements which were not a part of the regular low-flow data program are designated as miscellaneous sites. The partial-record and miscellaneous low-flow data are included in Appendix I. 
The locations of the sites where low-flow data were collected are shown in figure 1. Stations included in a continuing data collection program are referenced by their downstream order number, omitting the 2-digit prefix, as all gaging stations in Oklahoma have the lower Mississippi River basin prefix 07. This numbering system is compatible with U.S. Geological Survey published data reports. Miscellaneous sites are not in the official downstream order number system but are listed sequentially from 1 to 56. Figure 2 is a bar graph showing the type and period of record of each data collection site. The data collection sites are indexed both alphabetically by name and numerically by station number in Appendix II.

\section{METHOD OF ANALYSIS}

Analysis of streamflow data in this report generally follows established techniques in hydrology. The study involved defining low-flow frequency curves for continuous-record stations. The curves were based upon the probability of not exceeding a given discharge averaged over a certain period of time. Measurements at partial-record and miscellaneous sites were correlated with the daily mean discharge for the same day at a nearby continuous-record site. The miscellaneous or partial-record station discharge was then assigned the same frequency as the corresponding continuous-record station discharge.

\section{Low-F1ow Frequency}

Low-flow frequency involves two principles, nonexceedance probability and flow duration. Nonexceedance probability is a measure of the likelihood that a given flow rate will not be exceeded. Flow duration is the period of time over which a flow rate is averaged.

Flow duration was computed in days. For example, if the daily average flow rate is used, the flow duration is one day; a weekly average is a 7-day duration. The lowest values obtained from a particular duration for each year is used as the data entry for that year. If the lowest average value is computed for each of a number of years, a probability of that value not being exceeded in any given year may be estimated.

Nonexceedance probability computation consists of first ranking the lowest annual data entries according to magnitude with the smallest as number one. Then the probability of nonexceedance of the magnitude of that data entry is equal to the rank $M$ over the total number of data entries $\mathrm{N}$ plus one $(\mathrm{M} / \mathrm{N}+1)$. The inverse of the computed probability is the recurrence interval in years. Recurrence interval is the average number of years between flow occurrances or flow frequency. A smooth curve through a plot of the relation between annual low flows of a given duration and their corresponding recurrence interval represents the lowflow frequency curve for that duration. A set of frequency curves for a number of durations describes the low-flow characteristics of the stream. 
Figure 2.--Summary of streamflow records.

Note: Solid bars represent unregulated record, patterned bars represent regulated record and dotted bars represent miscellaneous measurements.

Station Name

Arkansas River at Ralston, Ok1a.

Arkansas River at Tulsa, Okla.

Arkansas River near Muskogee, Okla.

Arkansas River near Sallisaw, Okla.

Barnitz Creek near Arapaho, Okla.

Baron Fork at Eldon, Okla.

Bear Creek near Fallis, Okla.

Beaver Creek (Sand) Creek near Texhoma, Okla. Beaver Creek near Waurika, Okla.

Beaver River at Beaver, Okla.

Beaver River at Guymon, Ok1a.

Bellcow Creek at Chandler, Okla.

Bent Creek near Richmond, Okla.

Bent Creek near Seiling, Okla.

Big Cabin Creek near Big Cabin, Okla.

Big Cabin Creek near Pyramid Corners, Okla.

Bird Creek at Avant, Okla.

Bird Creek near Sperry, Okla.

Eitter Creek near Chickasha, Okla.

Black Bear Creek at Pawnee, Ok la.

Blue Beaver Creek near Cache, Okla.

Blue Creek near Connerville, Okla.

Biue Creek at Milburn, Okla.

Blue kiver near Blue, Okla.

Bluff Creek above Lake Hefner near Oklahoma City, okla.

Bols d'Ara Creek near Ponca City, Okla.

Buck Creek near Meyers, Jkla.

Duffalo Cosk near jovedato, chia.

Byrd's Mill Spring near Fittstown, Ok1a.

Caddo Creek near Ardmore, Okla.

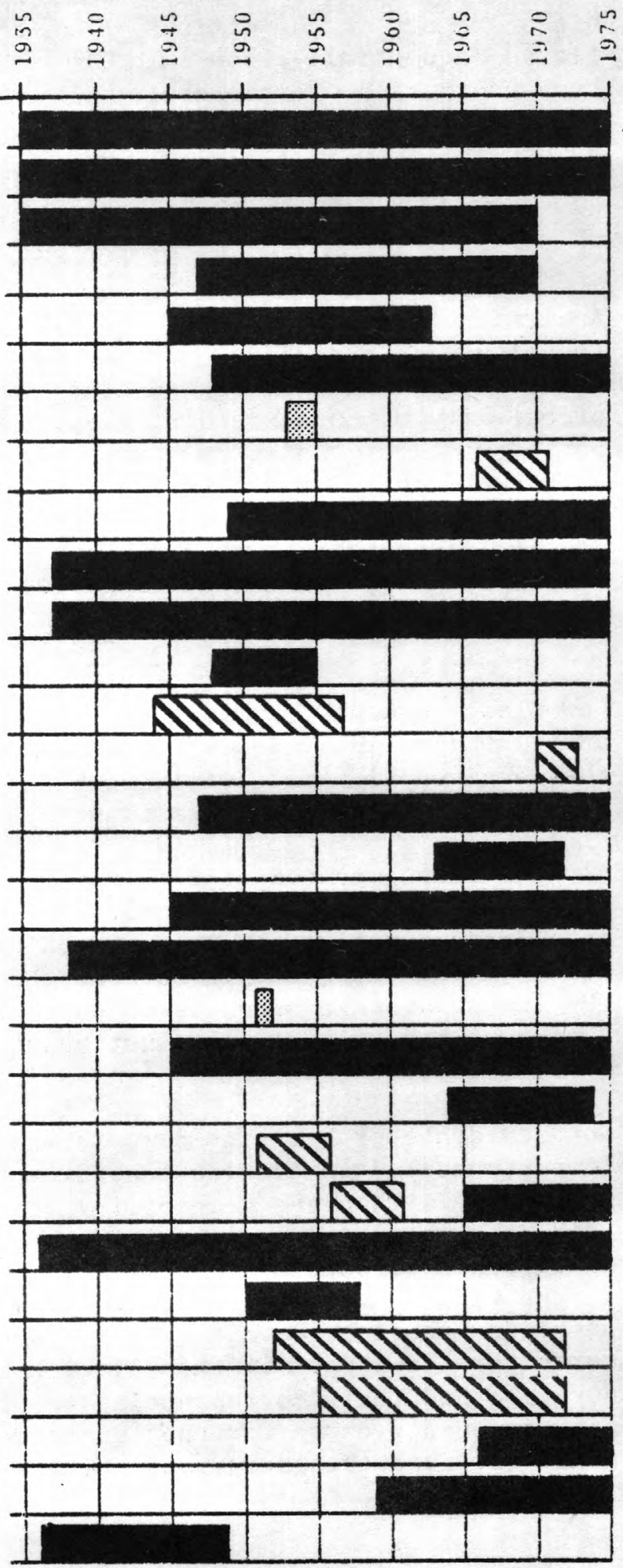


Figure 2.--Summary of streamflow records.--Continued

\section{Station Name}

Canadian River at Bridgeport, Okla.

Canadian River at Calvin, Okla.

Canadian River near Newcastle, Okla.

Canadian River near Nob1e, Ok1a.

Canadian River near Whitefield, Okla.

Caney Creek near Copan, Okla.

Caney River near Bartlesville, Okla.

Caney River near Hulah, Okla.

Caney River near Ochelata, Okla.

Caney River near Ramona, Okla.

Captain Creek near Wel1ston, Okla.

Cedar Creek near Carnegie, Okla.

Chickasaw Creek near Stringtown, Okla.

Chikaskia River near Blackwe11, Ok1a.

Cimarron River at Mannford, Okla.

Cimarron River at Oilton, Okla.

Cimarron River at Perkins, Okla.

Cimarron River near Buffalo, Okla.

Cinarron River near Guthrie, Ok1a.

Cimarron River near Kenton, Okla.

Cimarron River near Mocane, Okla.

Cimarron River near Waynoka, Okla.

Clear Boggy Creek near Caney, Okla.

Clear Boggy Creek near Frisco, Okla.

Clear Boggy Creek near Tupe1o, Okla.

Clear Creek near Elmwood, Okla.

Clear Creek near May, Okla.

Lobb ureek near fort loob, okla.

Co1d Springs Creek near Castaneda, Okla. Coldwater Creek near Hardesty, Okla.

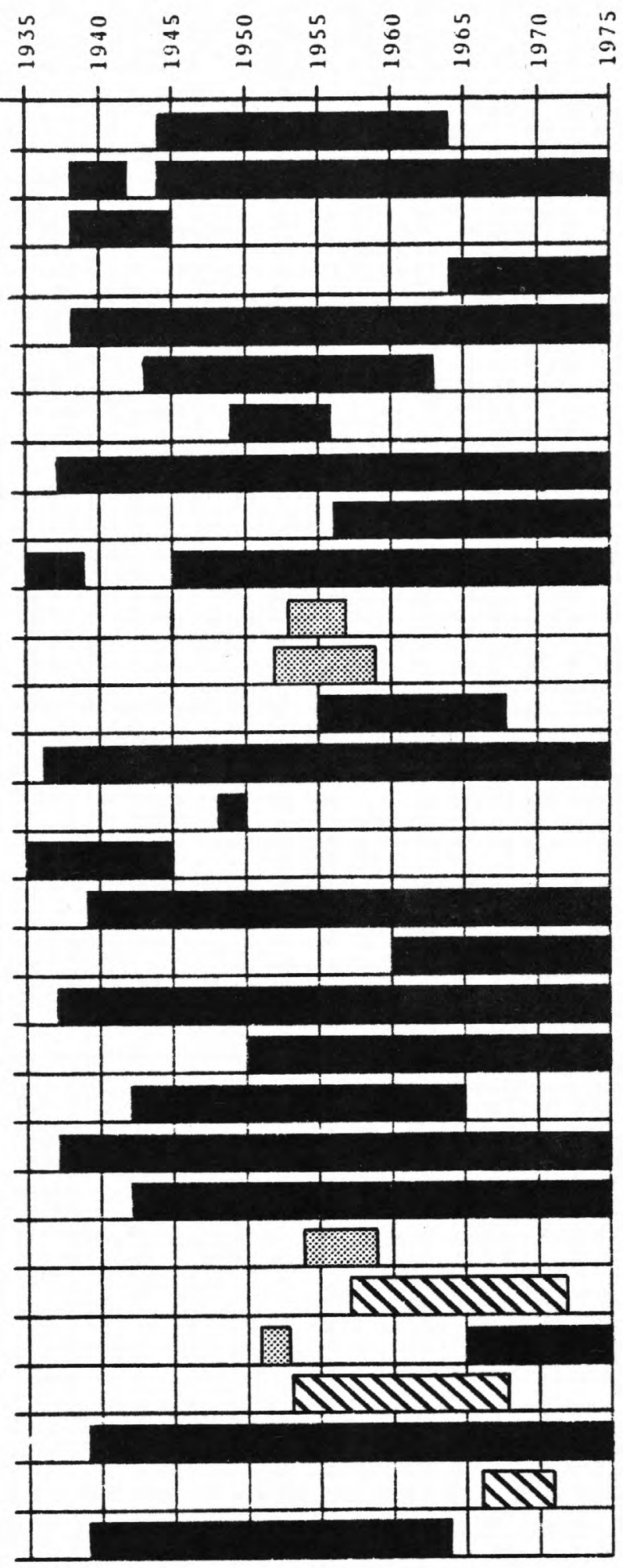


Figure 2.--Summary of streamflow records.--Continued

Station Name

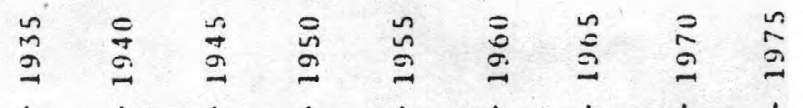

Commission Creek near Grand, Okla.

Cottonwood Creek near Guthrie, Okla.

Cottonwood Creek near Navina, Ok1a.

Cottonwood Creek near Seward, Okla.

Council Creek near Stillwater, Ok1a.

Cow Creek near Waurika, Okla.

Deep Fork near Beggs, Okla.

Deep Fork near Dewar, Okla.

Deep Fork near Welty, Okla.

Deep Red Run near Randlett, Okla.

Deer Creek near Edmond, Okla.

Deer Creek at Hydro, Okla.

Deer Creek near Tonkawa, Okla.

Delaware Creek near Wapunucka, Okla.

Dirty' Creek near Warner, Okla.

Dry Creek near Kendrick

Duck Pond Creek near Clear Lake, Okla.

Eagle Chief Creek near Aline, Okla.

Eagle Chief Creek near Carmen, Okla.

Eagle Chief Creek near Cleo, Okla.

East Branch Sandstone Creek near Elk

City, Okla.

East Cache Creek near Walters, Okla.

Elk Creek near Hobart, Okla.

Elm Fork of North Fork Red River near Carl, Okla.

Elm Fork of North Fork Red River near

Mangum, Okla.

Finn Creek near Story, okla.

Five Mile Creek near Eakley, Okla.

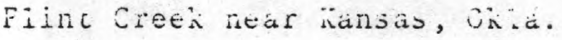

Fourche Maline near Red Oak, Okla.

Gaines Creek near Krebs, Okla.

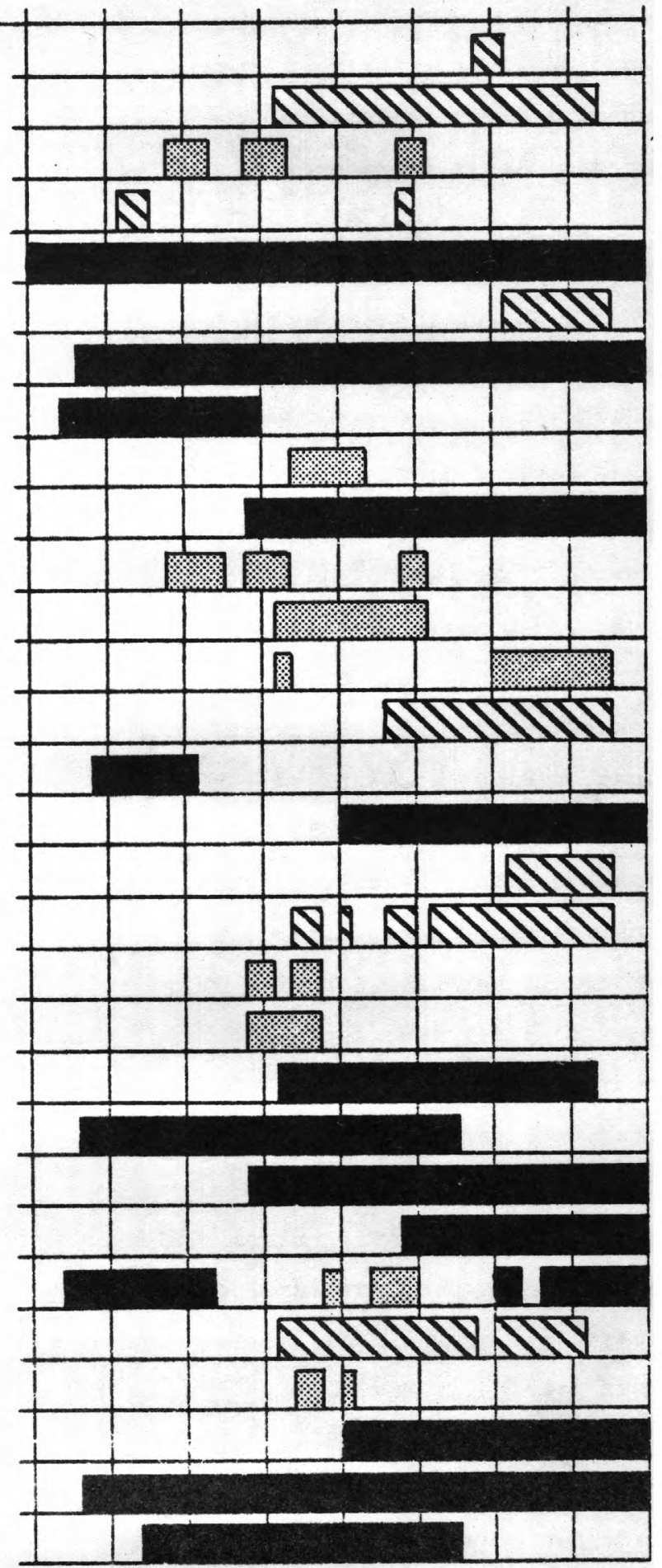


Figure 2.--Summary of streamflow records.--Continued

Station Name

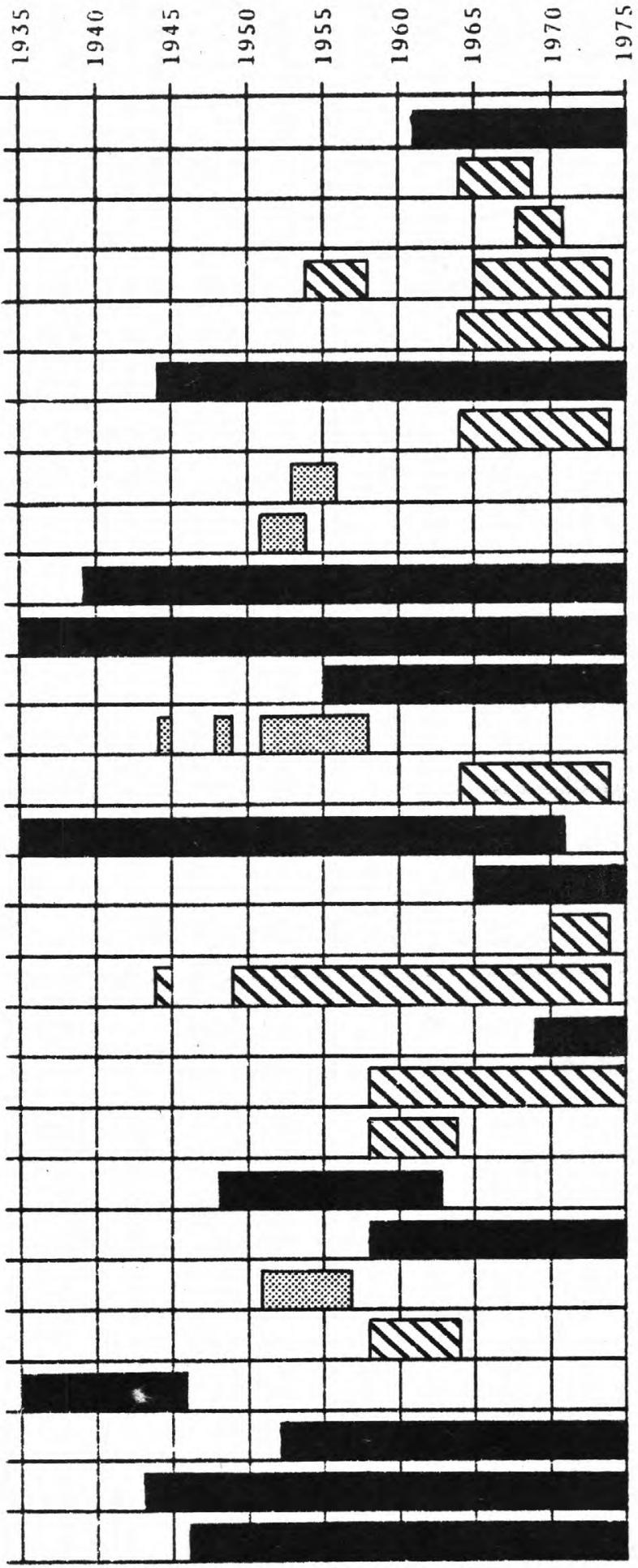

Little River below Lukfata Creek near Idabe 1, Okia. 
Figure 2.--Summary of streamflow records.--Continued

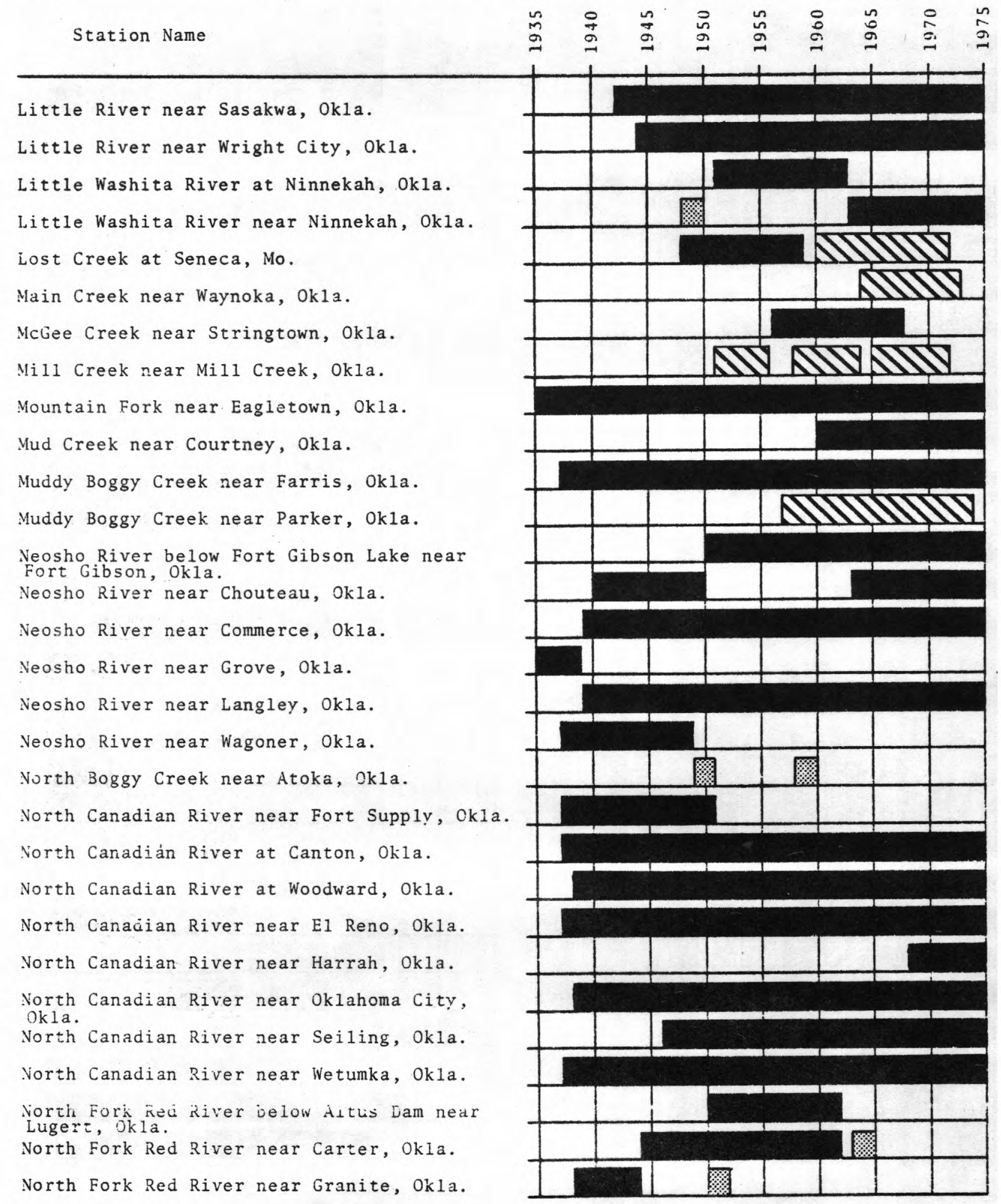


Figure 2.--Summary of streamflow records.--Continued

Station Name

North Fork Red River near Headrick, Okla.

North Fork Red River near Texola, Okla.

Otter Creek at Mountain Park, Okla.

Palo Duro Creek near Range, Okla.

Pennington Creek near Reagon, Okla.

Persimmon Creek (North) near Sharon, Okla.

Persimmon Creek (South) near Sharon, Okla.

Persimmon Creek near Mutual, Okla.

Polecat Creek below Heyburn Lake near Heyburn, Ok1a.

Pond Creek near Lamont, Okla.

Pony Creek near Optima, Okla.

Poteau River at Poteau, Okla.

Poteau River near Wister, Okla.

Preacher Creek near Dover, Okla.

Pryor Creek near Pryor, Okla.

Quapaw Creek near Meeker, Ok1a.

Rainy Mountain Creek near Mountain View, Okla.

Red River at Arthur Cicy, Tex.

Red River near Colbert, Okla.

Red River at Denison Dam near Denison, Tex.

Red River near Terral, Okla.

Red Rock Creek near Red Rock, Okla.

Rock Creek at Dougherty, Okla.

Roundup Creek near Woodward, Okla.

Rush Creek at Purdy, Okla.

Rush Creek near Maysville, Okla.

Salina Creek near Salina, Okla.

Sallisaw Creek near Sallisaw, Okla.

Salt Creek near Dewright, Okla.

Salt Creek near Okeene, Okla.

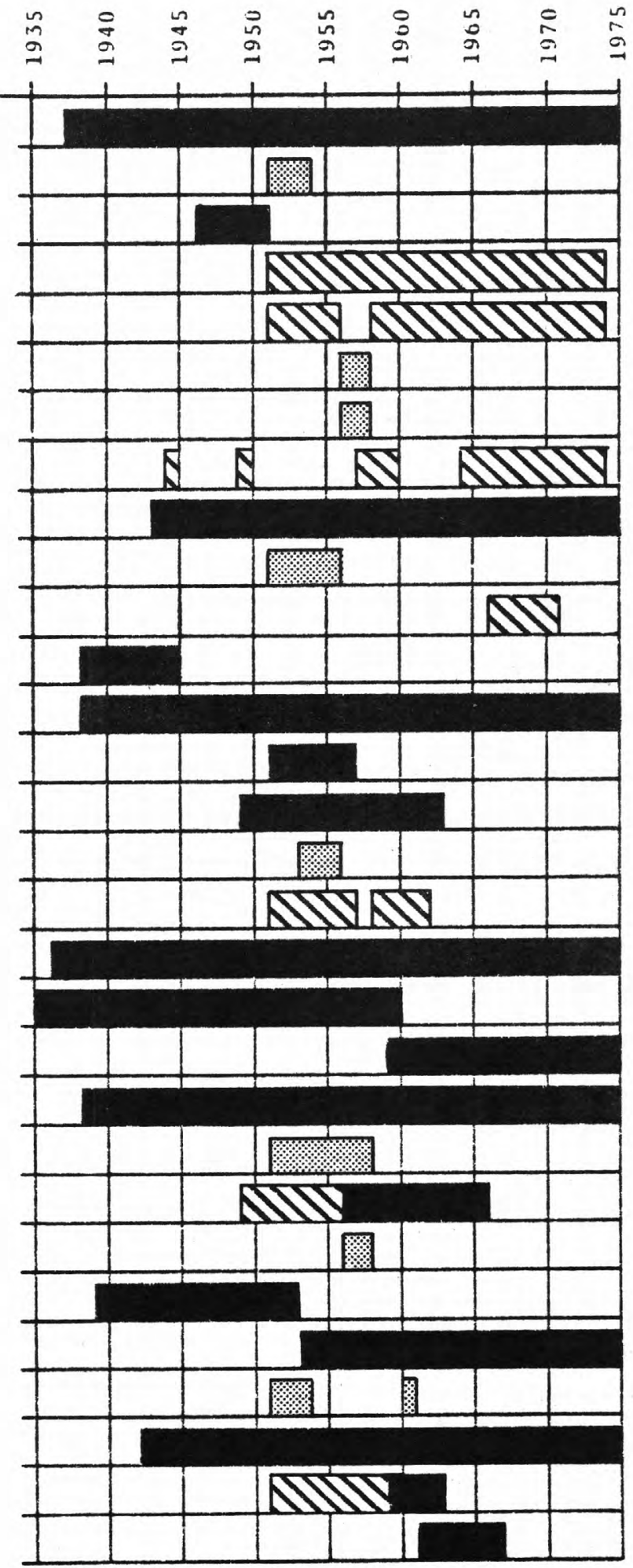


Figure 2.--Summary of streamflow records.--Continued

Station Name

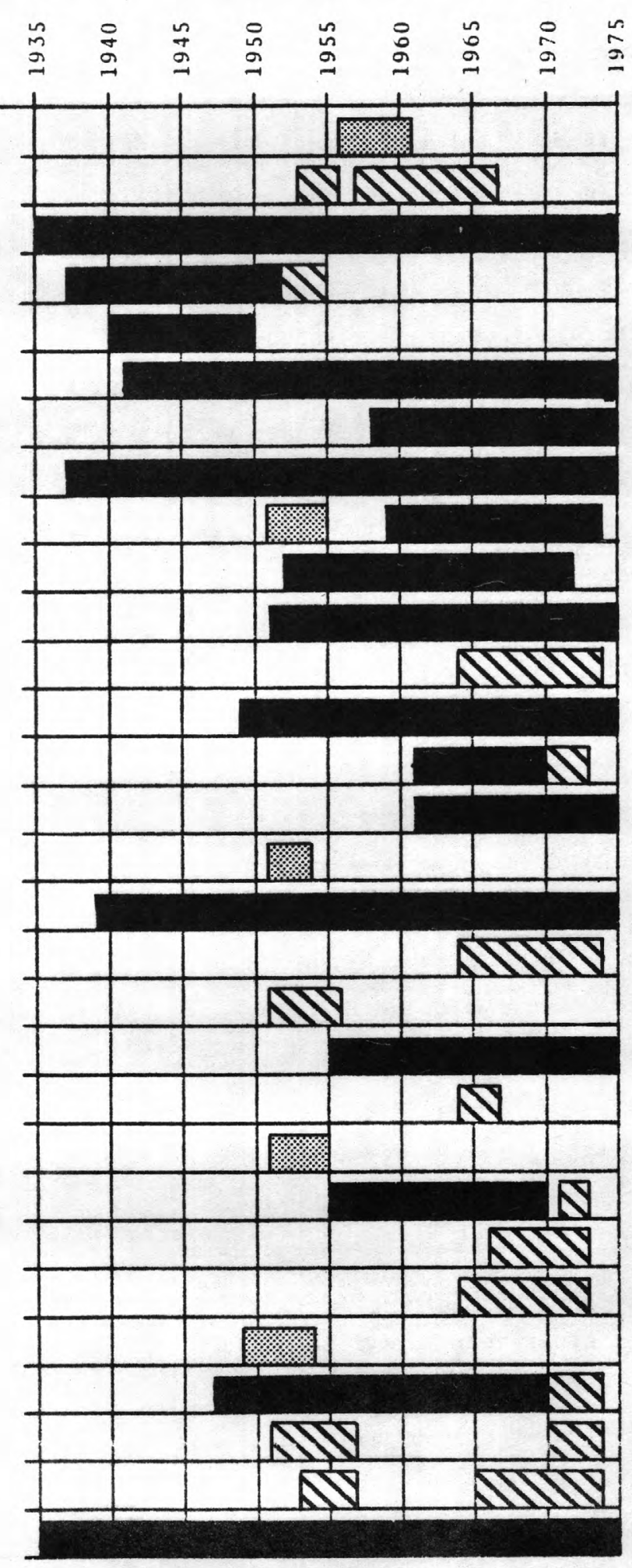

Salt Creek near Pearson, Okla.

Salt Creek near Shidler, Okla.

Salt Fork Arkansas River at Tonkawa, Okla.

Salt Fork Arkansas River near Alva, Okla.

Salt Fork Arkansas River near Cherokee, Okla.

Salt Fork Arkansas River near Jet, Okla.

Salt Fork Arkansas River near Winchester, Okla.

Salt Fork Red River at Mangum, Okla.

Sand Creek at Okesa, Okla.

Sandstone Creek near Berlin, Okla.

Sandstone Creek near Cheyenne, Okla.

Sandy Creek near Bryan, Okla.

Skeleton Creek near Love11, Okla.

Snake Creek near Bixby, Okla.

Spavinaw Creek near Sycamore, Ok1a.

Spring Creek near Ada, Okla.

Spring River near Quapaw, Okla.

Starvation Creek near Prentiss, Okla. Stinking Creek near Carnegie, Okla.

Sugar Creek near Gracemont, Okla.

Suttle Creek near Davidson, Okla.

Sweetwater Creek near Texola, Okla.

Tenmile Creek near Miller, Okla.

Tepee Creek near Eva, Okla.

Turkey Creek near Carmargo, Okla.

Turkey Creek near Dover, Okla.

Turkey. Creek near Drummond, Okla.

Turkey Creek near Olustee, Okla.

Turkey Creek near Sayre, Okla.

Verdigris River near Claremore, Okla. 
Figure 2.--Summary of streamflow records.--Continued

Station Name

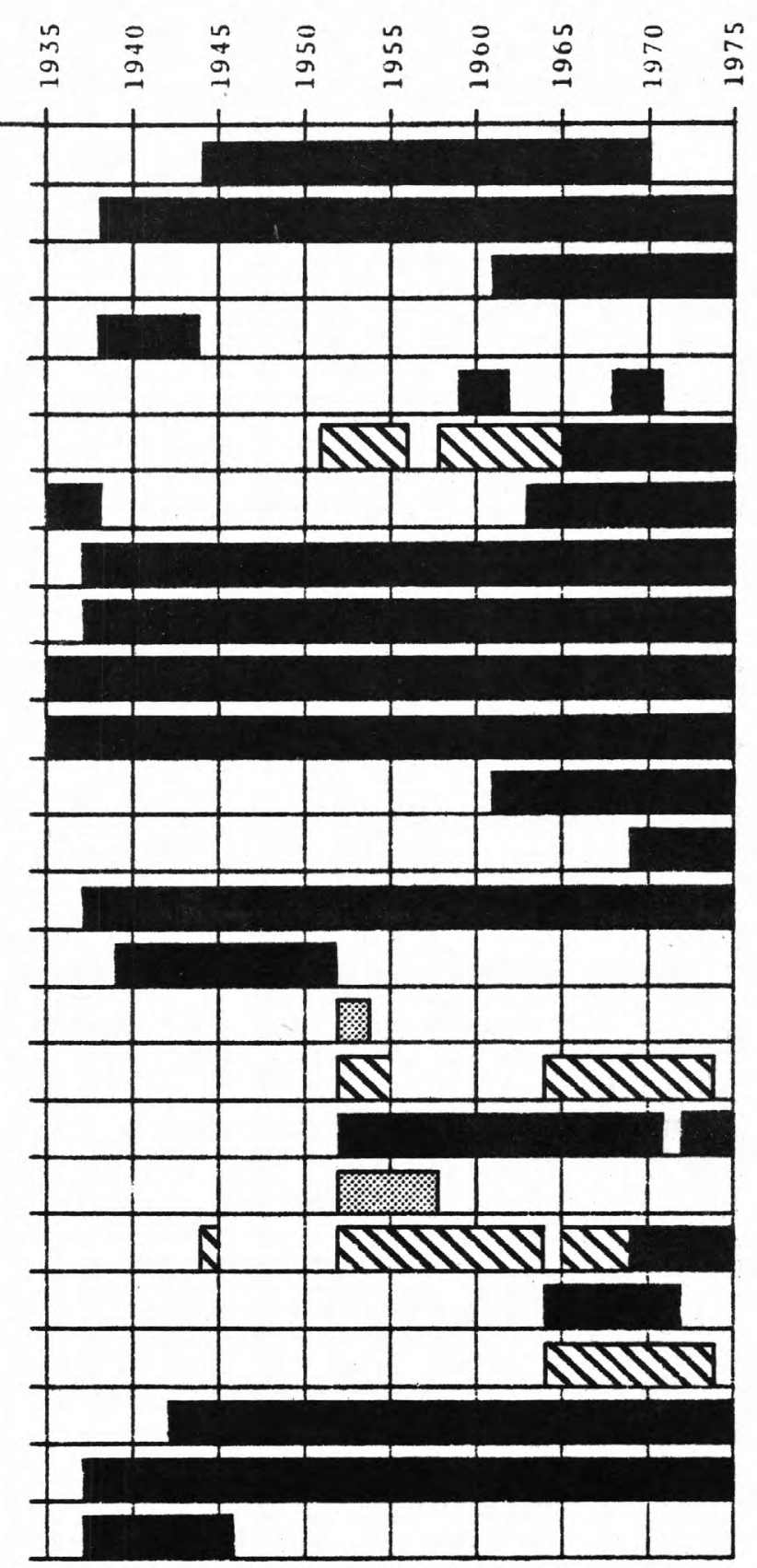

West Otter Creek at Snyder Lake near Mountain Park, Okla.

Wewoka Creek near Wetumka, Okla.

Wildhorse Creek near Hoover, OkIa.

Wildhorse (Sand) Creek near Pond Creek, Ok

Winter Creek near Alex, Okla.

Wolf Creek near Fargo, Okla.

Wolf Creek near Fort Supply, Okia.

Wolf Creek near Shattuck, Okla. 


\section{at Continuous-Record Stations}

Low-flow frequency curves were developed for sites where sufficient continuous streamflow record was available. Curves were developed for 1-day, 7-day, 14-day, and 30-day durations. The low-flow duration computation and annual ranking of the streamflow data was done for continuous records collected through 1974 in a previous study by Mize (1975). Ranked low-flow discharges from Mize's study were plotted against their recurrence intervals to obtain low-flow frequency curves. Discharges corresponding to the 2-year, 10-year and 20-year recurrence intervals were selected from the curves. These recurrence interval low flows at the 1-, 7-, 14-, and 30-day durations discussed above are published later in this report for all continuous record sites which have sufficient data to define the frequency curves adequately.

The frequency analysis for this study was done graphically. Theoretical methods of fitting data to predefined distributions are available and more convenient to use, but they may not fit low-flow data (Riggs, 1972). After several attempts at using theoretical distributions, it was apparent that graphical analysis was a more appropriate approach for Okalhoma streams.

Table 1 and figure 3 show an example of graphical frequency analysis for Clear Boggy Creek near Caney, Oklahoma.

Computation of Low-Flow Characteristics
at Partial-Record and Miscellaneous Sites

Sites with insufficient data to develop low-flow frequency curves were related to continuous-record sites. The partial-record or miscellaneous site must have enough discharge measurements to establish a correlation between it and a continuous-record site. Oklahoma has comparatively few suitable continuous-record sites, resulting in many miscellaneous and partial-record sites being correlated to the same continuous-record site. Therefore, a select few continuous-record sites over the State were used as index stations for correlation with nearby partial-record and miscellaneous sites.

The index stations selected in this study were required to meet several criteria with respect to their geographic location and streamflow record. An index station must represent a specific area of the State with respect to topographic and geologic conditions that may have an effect on low flow and have no major regulation or other manmade changes in the drainage basin. Also, the index stations must have the same periods of record as the partial-record and miscellaneous sites. An attempt was made to use streams of relatively small drainage area to avoid incorporating many varied topographic and geologic factors into one record. The index sites must be less ephemeral than any partial record or miscellaneous site. With all these criteria taken into account, many continuous-record sites were eliminated as index stations. Where index stations did not have periods of record compatible with all partial-record and miscellaneous sites, more than one index station was used in the same general location. 
Table 1.--Lowest mean discharge and ranking for Clear Boggy Creek near Caney, Okla

\begin{tabular}{|c|c|c|c|c|c|c|}
\hline \multirow[t]{2}{*}{ Rank } & \multirow{2}{*}{$\begin{array}{l}\text { Non- } \\
\text { exceedance } \\
\text { probability }\end{array}$} & \multirow{2}{*}{$\begin{array}{c}\text { Recurrence } \\
\text { interval }\end{array}$} & \multicolumn{4}{|c|}{$\begin{array}{l}\text { Lowest mean flow for } \\
\text { given duration in } \mathrm{ft}^{3} / \mathrm{s}\end{array}$} \\
\hline & & & 1 day & 7 day & 14 day & 30 day \\
\hline 1 & 0.031 & 32.00 & 0.00 & 0.00 & 0.00 & 0.00 \\
\hline 2 & .062 & 16.00 & .0 & .00 & .00 & .01 \\
\hline 3 & .093 & 10.67 & .0 & .00 & .04 & .56 \\
\hline 4 & .125 & 8.00 & .39 & .59 & .89 & 1.04 \\
\hline 5 & .156 & 6.40 & .40 & .66 & 1.02 & 2.45 \\
\hline 6 & .187 & 5.33 & .40 & .90 & 1.16 & 2.57 \\
\hline 7 & .218 & 4.57 & .80 & 1.00 & 1.31 & 4.73 \\
\hline 8 & .250 & 4.00 & 1.60 & 2.10 & 3.51 & 5.33 \\
\hline 9 & .281 & 3.56 & 3.80 & 4.89 & 7.39 & 8.28 \\
\hline 10 & .312 & 3.20 & 3.90 & 6.23 & 7.47 & 8.68 \\
\hline 11 & .343 & 2.91 & 5.70 & 6.43 & 7.6 & 10.10 \\
\hline 12 & .375 & 2.67 & 5.90 & 6.87 & 9.21 & 12.70 \\
\hline 13 & .406 & 2.46 & 6.60 & 8.24 & 9.54 & 12.80 \\
\hline 14 & .437 & 2.29 & 8.10 & 8.99 & 10.10 & 13.50 \\
\hline 15 & .468 & 2.13 & 8.80 & 9.34 & 10.90 & 13.80 \\
\hline 16 & .500 & 2.00 & 8.80 & 9.44 & 11.30 & 14.40 \\
\hline 17 & .531 & 1.88 & 9.00 & 9.93 & 12.10 & 16.10 \\
\hline 18 & .562 & 1.78 & 10.00 & 10.90 & 12.30 & 16.20 \\
\hline 19 & .593 & 1.68 & 10.00 & 11.70 & 13.10 & 16.40 \\
\hline 20 & .625 & 1.60 & 11.00 & 11.90 & 13.20 & 16.90 \\
\hline 21 & .656 & 1.52 & 11.00 & 12.00 & 13.40 & 22.30 \\
\hline 22 & .687 & 1.45 & 11.00 & 12.60 & 13.50 & 22.40 \\
\hline 23 & .718 & 1.39 & 12.00 & 12.70 & 14.20 & 23.80 \\
\hline
\end{tabular}


Table 1.--Lowest mean discharge and ranking for Clear Boggy Creek near Caney, Okla Continued

\begin{tabular}{|c|c|c|c|c|c|c|}
\hline \multirow[t]{2}{*}{ Rank } & \multirow{2}{*}{$\begin{array}{l}\text { Non- } \\
\text { exceedance } \\
\text { probability }\end{array}$} & \multirow{2}{*}{$\begin{array}{c}\text { Recurrence } \\
\text { interval }\end{array}$} & \multicolumn{4}{|c|}{$\begin{array}{l}\text { Lowest mean flow for } \\
\text { given duration in } \mathrm{ft}^{3} / \mathrm{s} \text {. }\end{array}$} \\
\hline & & & 1 day & 7 day & 14 day & 30 day \\
\hline 24 & 0.750 & 1.33 & 12.00 & 13.60 & 14.40 & 25.50 \\
\hline 25 & .781 & 1.28 & 13.00 & 13.60 & 14.90 & 28.10 \\
\hline 26 & .812 & 1.23 & 13.00 & 13.90 & 17.60 & 28.80 \\
\hline 27 & .843 & 1.19 & 21.00 & 21.00 & 21.40 & 30.40 \\
\hline 28 & .875 & 1.14 & 22.00 & 23.10 & 25.20 & 44.20 \\
\hline 29 & .906 & 1.10 & 25.00 & 31.30 & 28.10 & 45.70 \\
\hline 30 & .937 & 1.07 & 35.00 & 36.70 & 38.70 & 54.90 \\
\hline 31 & .968 & 1.03 & 36.00 & 42.00 & 44.50 & 118.00 \\
\hline
\end{tabular}




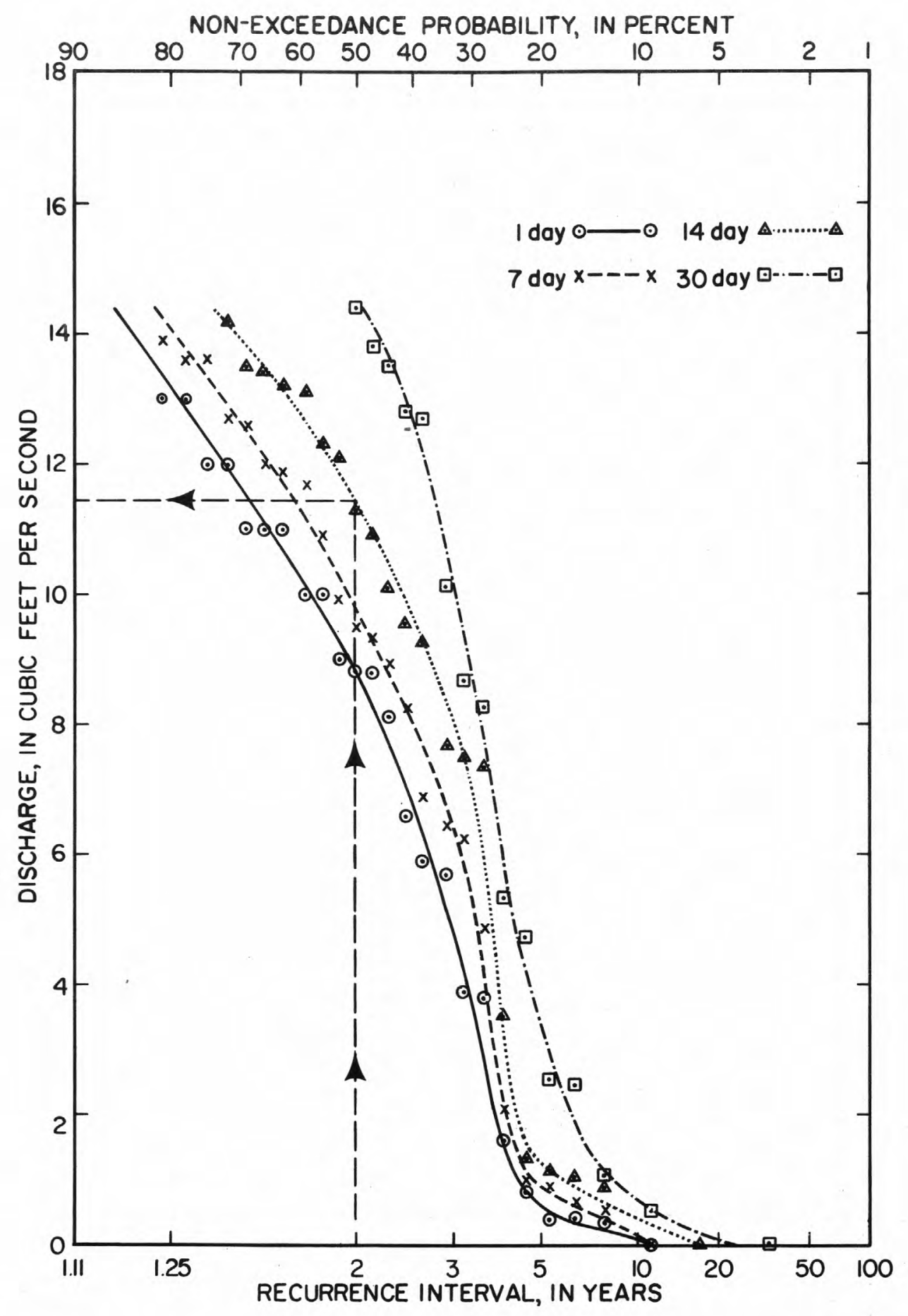

Figure 3.--Low-flow frequency curves for selected durations for Clear Boggy Creek near Caney, Okla. 
After the index stations were selected, the Thiessen polygon method was used to regionalize each index station. The regional polygons were distorted in some cases so the polygons would all be reasonably homogeneous in topography and geology. The location of the index station and their regional polygons are shown in figure 4.

The discharge measurements at all miscellaneous and partial-record sites were plotted aginst the corresponding daily discharge on the same date at the index stations in their regional polygon. A smooth regression line was drawn through the plotted points as shown on the graph in figure 5. At many sites the plotted points were scattered over the graph, giving no correlation; thus, no low-flow estimates could be made.

Using the dashed lines on the low-flow frequency curves of the index station as shown in the example graph in figure 3 for Clear Boggy Creek, the discharge for a given frequency is determined. Then the dashed line on the correlation analysis in figure 5 is used to determine the corresponding discharge frequency at the partial-record or miscellaneous site.

\section{STUDY RESULTS}

Tables of selected low-flow characteristics are included in this section. Also, the expected accuracy of the analysis is discussed.

\section{Low-Flow Characteristics of Selected Streams}

Table 2 includes selected low-flow characteristics for all streams analyzed in this study. Stream names and numbers have been compiled for the users convenience.

\section{Discussion of Errors}

Sources of error in statistical analysis related to hydrology are many and varied, because of the many interpretations and extraplations of the original data. For any hydrologic analysis to have meaningful application to practical problems, knowledge of the errors involved in its use are essential. Therefore, the types and magnitudes of significant errors that can be expected when using the results of this study are briefly discussed.

Many sources of error exist in a low-flow analysis. First, there is a time-sampling error in the original data which varies in magnitude depending upon the length of record and variability of data at a particular site being analyzed. Secondly, the low-flow frequency curves plotted for the continuous-record sites have errors involved in their development. And finally, errors involved in correlating the partial-record and miscellaneous sites with the index stations must be recognized. When all these errors are accounted for and compared to the magnitude of the low flows, error percentages may be high. 


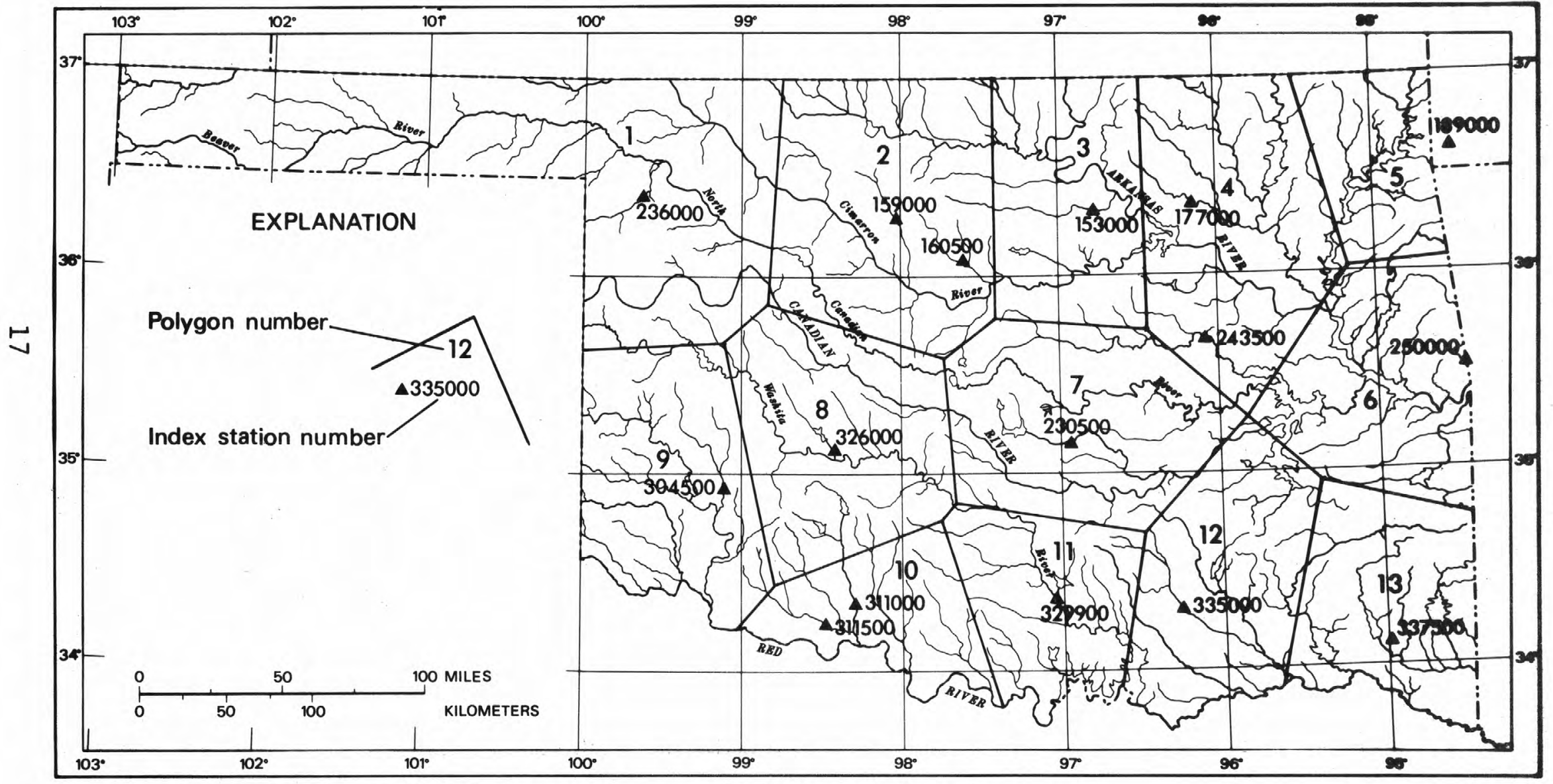

Figure 4.--Location of index stations and their regional polygon boundaries. 


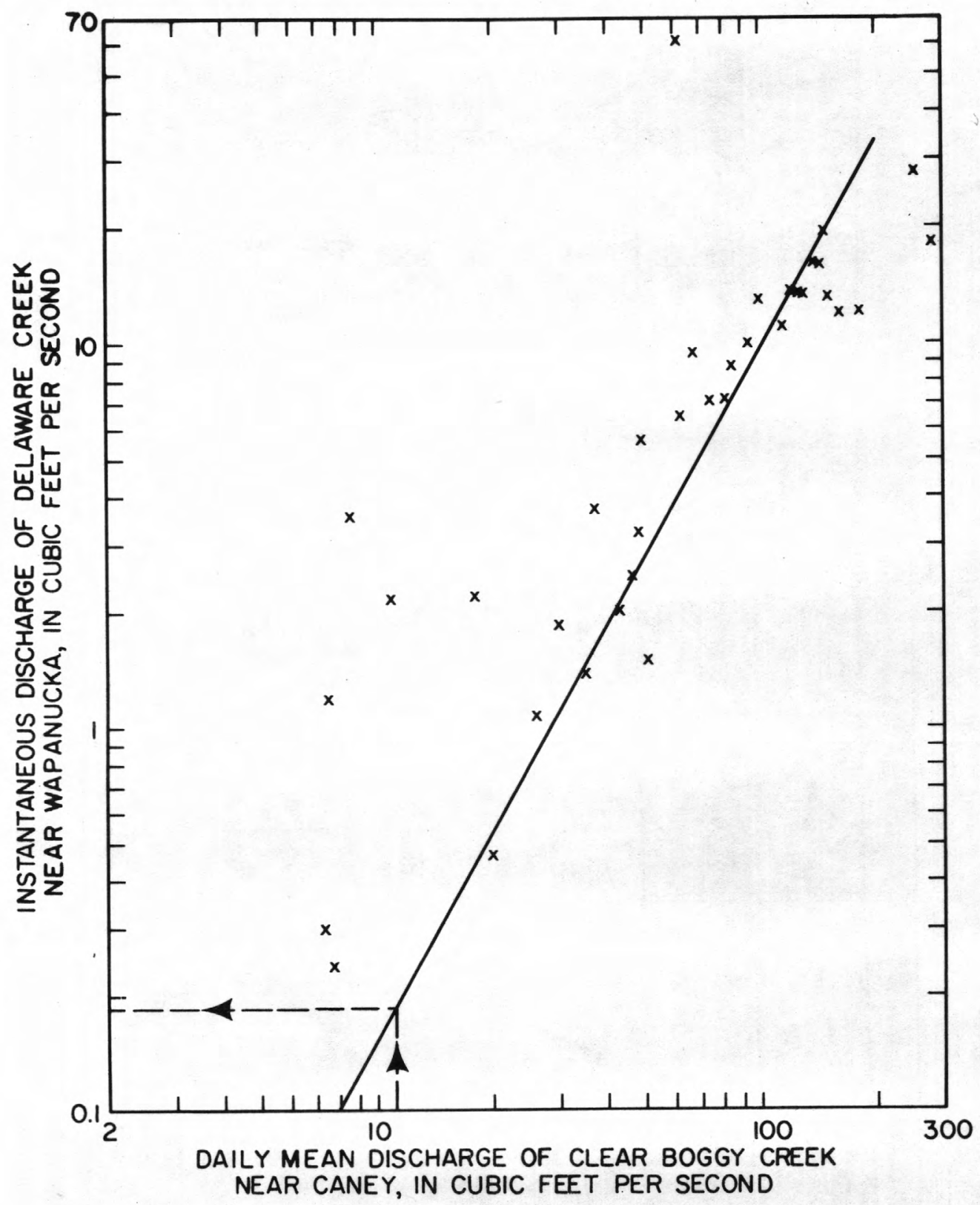

Figure 5.--Example of low-flow analysis to obtain low-flow characteristics at a partialrecord or miscellaneous site. 


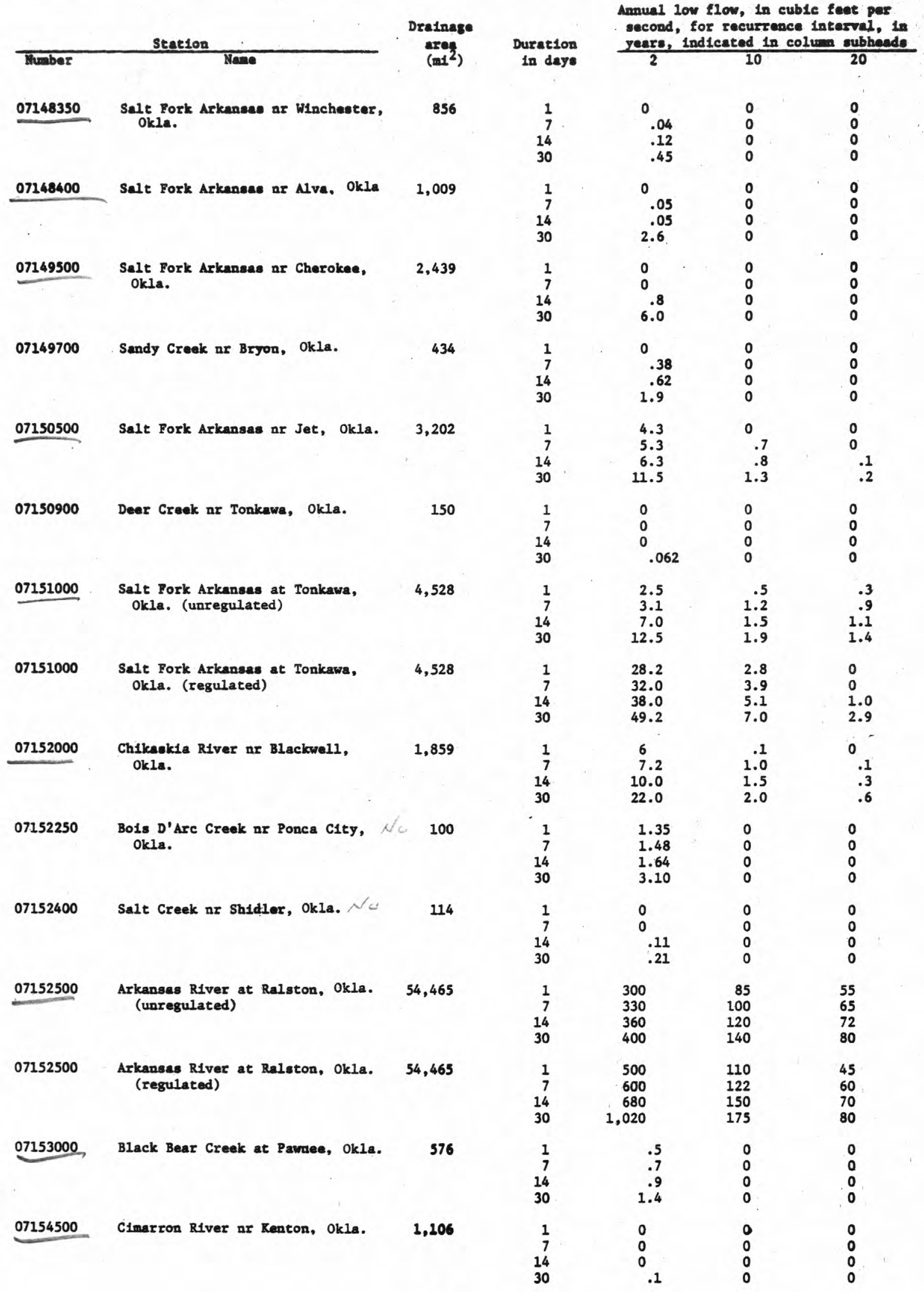


Table 2.--Selected low-flow characteristics of streams in Oklahoma--Continued

Annual lov flow, in cubic feet per second, for recurrence interval, in

\begin{tabular}{|c|c|c|}
\hline & Station & $\begin{array}{c}\text { Drainage } \\
\text { area }\end{array}$ \\
\hline Number & Name & $\left(m 1^{2}\right)$ \\
\hline 07155000 & $\begin{array}{l}\text { Cimarron River above Ute Creek } \\
\text { nr Boise C1ty, Okla. }\end{array}$ & 1,955 \\
\hline 07157000 & Cimarron River nr Mocane, Okla. & 8,670 \\
\hline 07157950 & Cimarron RIver nr Buffalo, Okla. & 11,930 \\
\hline 07157960 & Buffalo Creek nr Lovedale, Okla. & 408 \\
\hline 07158000 & Cimarron River nr Waynoka, Okla. & 13,334 \\
\hline 07158100 & Eagle Chief Creek nr Aline, Okla. & 4606 \\
\hline 07158400 & Salt Creek nr Okeene, Okla. & 196 \\
\hline
\end{tabular}
years, indicated in column subheads In days

1
7
14
30

1
7
14
30

30

$\begin{array}{lll}0 & 0 & 0 \\ 0 & 0 & 0 \\ 0 & 0 & 0 \\ 0 & 0 & 0 \\ 12 & 2.5 & .6 \\ 18.5 & 4.5 & 2.5 \\ 22.5 & 7.6 & 4.8 \\ 28.5 & 10.0 & 6.3\end{array}$

07158500 Preacher Creek nr Dover, Okla.

14.5

07159000 Turkey Creek nr Drummond, Okla.

$\begin{array}{ll}0 & 0 \\ 0 & 0 \\ 0 & 0 \\ 0 & 0\end{array}$

\section{0}

$\begin{array}{ll}0 & 0 \\ 0 & 0 \\ 0 & 0 \\ 0 & 0\end{array}$

$\begin{array}{ccc}0 & 0 & 0 \\ .1 & 0 & 0 \\ .6 & 0 & 0 \\ 7.5 & .4 & 0\end{array}$

$\begin{array}{lll}2.1 & 0 & 0 \\ 3.9 & 0 & 0 \\ 4.6 & .48 & 0 \\ 6.5 & 1.0 & .27\end{array}$

$\begin{array}{lll}0 & 0 & 0 \\ 0 & 0 & 0 \\ 0 & 0 & 0 \\ 0 & 0 & 0 \\ 0 & 0 & 0 \\ 0 & 0 & 0 \\ 0 & 0 & 0 \\ .2 & .1 & 0\end{array}$

07159500 Bluff Creek above Lake Hefner $\mathrm{nr} \quad 1.62$ Oklahoma City, Okla.

$07159800 \quad$ Cottonwood Creek nr Guthrie, Okla. N/6 366

07160000 Cimarron River nr Guthrie, Okla. 16,892

$07160500 \quad$ Skeleton Creek nr Lovell, Okla. 410

07161000 Cimarron River at Perkins, Okla. 17,852

07163000, Counc11 Creek nr Stillwater, 0k1a. 31

$\begin{array}{lll}0 & 0 & 0 \\ 0 & 0 & 0 \\ 0 & 0 & 0 \\ 0 & 0 & 0\end{array}$


Table 2.--Selected low-flow characteristics of streams in Oklahoma.--Continued

$\begin{array}{llcc}\text { Station } & \begin{array}{c}\text { Drainage } \\ \text { areg } \\ \text { Number }\end{array} & \begin{array}{c}\text { Duration } \\ \text { Name days }\end{array}\end{array}$

Annual low flow, in cublc feet per

second, for recurrence interval, in

years, indicated in column subheads

07163500 Cimarron RIver ar O1lton, Okla. 18,669

07164000 Cimarron River at Mannford, Okla. 18,849

07164500 Arkansas River at Tulsa, Okla. 74,615 (unregulated)

74,615

07164500 Arkansas River at Tulsa, Okla. 74,615

(regulated)
07165500 Polecat Creek below Heyburn Lake
nr Heyburn, Okla.
07165500 OPolecat Creek below Heyburn Lake
nr Heyburn, Okla.

4,615

50.0

1
7
14
30

42

51

74

10

$\begin{array}{ll}1 & 0 \\ 2 & 0 \\ 4 & 0 \\ 5 & 0\end{array}$

90

110

130
170

170

620
680

680
750

925

425

890

1,180

1,430

0

100

150

200

225

$\begin{array}{lr}150 & 60 \\ 310 & 300 \\ 380 & 300 \\ 460 & 300\end{array}$
07165550 Snake Creek nr B1xby, Okla.

0

0
0
0

$07171000 \quad$ Verd1gris River nr Lenapah, Okla. 3,639 '(unregulated)

18.9

22.4

28.7

33.6

$\begin{array}{ll}0 & 0 \\ 0 & 0 \\ 0 & 0 \\ 0 & 0\end{array}$

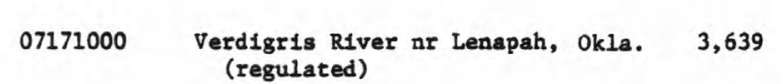

22.5

34.5

52.5

4,339

07171400

Verdigris RIver nr Oologah, Okla. (regulated)

1.5
16
34.5

0
0
0
0

0
0
0
0

(regulated)
$07171500 \quad \begin{gathered}\text { Verdigris River nr Sageeyah, Okla. } \\ \text { (regulated) }\end{gathered}$

07173000 Caney River nr Hulah, Okla.

34.5

0
0
0
0

0
0
0
0
(unregulated)
07173000 Caney RIver nr Hulah, Okla.
(regulated)
(unregulated)
$07173000 \quad \begin{gathered}\text { Caney River nr Hulah, Okla. } \\ \text { (regulated) }\end{gathered}$
(unregulated)
07173000 Caney River nr Hulah, Okla.
(regulated)

07174000 Caney Creek ar Copan, Okla.

424

$\begin{array}{lll}22 & 0 & 0 \\ 30 & 0 & 0 \\ 46 & 0 & 0 \\ 73 & 0 & 0\end{array}$

$\begin{array}{lll}1.53 & 0 & 0 \\ 2.2 & 0 & 0 \\ 2.7 & 0 & 0 \\ 4.25 & 0 & 0\end{array}$

$\begin{array}{lll}2.4 & .1 & 0 \\ 4.8 & .15 & .12 \\ 6.3 & .20 & .15 \\ 9.7 & 1.3 & .29\end{array}$

$\begin{array}{lll}.04 & 0 & 0 \\ .07 & 0 & 0 \\ .15 & 0 & 0 \\ .20 & 0 & 0\end{array}$


Table 2.--Selected low-flow characteristics of streams in Oklahoma.--Continued

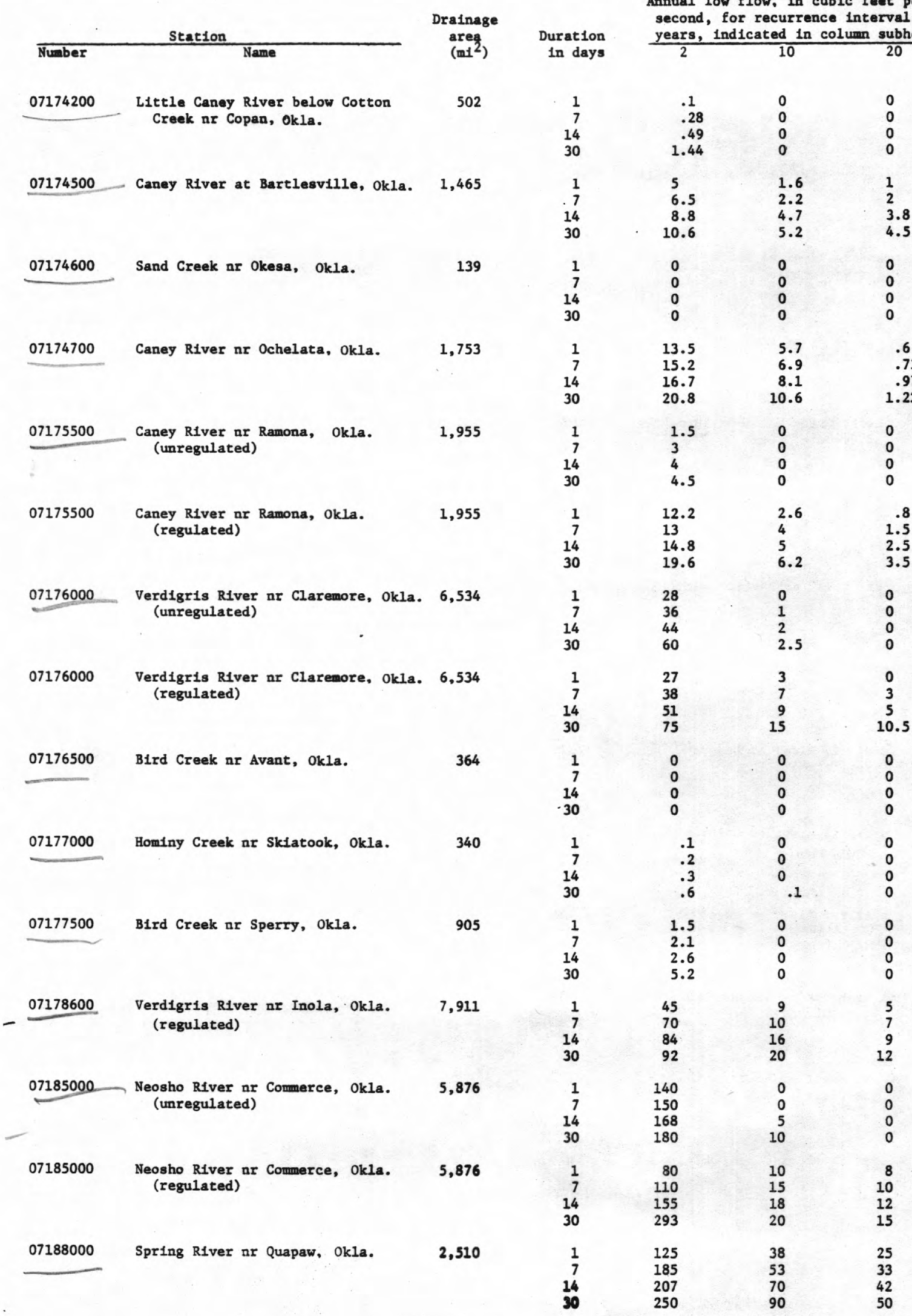


Table 2.--Selected 1ow-flow characteristics of streams in Oklahoma.--Continued

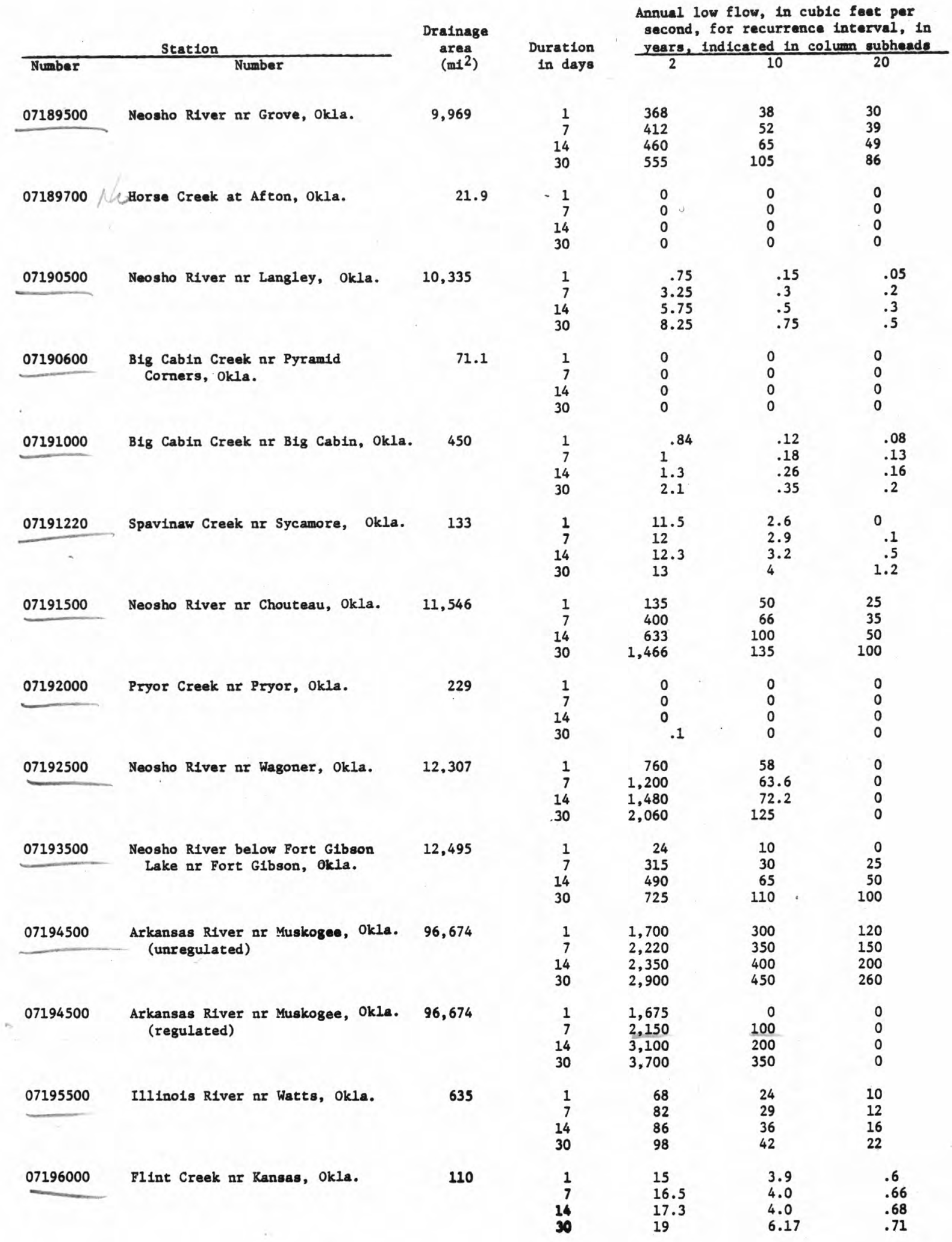


Table 2.--Selected low-flow characteristics of streams in 0klahoma.--Continued

$\frac{\text { Station }}{\text { Number }}$

07196500 , Illinois River nr Tahlequah, Okla.

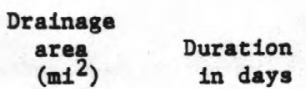

07197000 Baron Fork at Eldon, Okla.

307

07198000

Illinols River nr Gore, Okla. (unregulated)

1,626

07198000 Illinols River nr Gore, Okla. (regulated)

07198500 Dirty Creek nr Warner, Okla.

227

$07228150 / 0$ Commision Creek nr Grand, Okla.

07228400 Noer Creek at Hydro, Okla. $\underbrace{072800}_{\begin{array}{c}\text { Canadian River } \\ \text { (unregulated) }\end{array} \text { at Bridgeport, Okla. 25,229 }}$

07228500 Candian River at Bridgeport, Okla. 25,229 (regulated)

07229000 Canadian River nr Newcastle, Okla. 25,763

07229100 Canadian R1ver nr Noble, Okla. 25,911

07229300 Walnut Creek at Purce11, Okla. 202

07229300 Walnut Creek at Purcell, Okla. 202

07230000 Little River below Lake Thunder-

bird nr Norman, Okla. (urregulated)

257

07230000

Little River below Lake Thunderbird nr Norman, Okla. (regulated)

456

07230500 Little R1ver nr Tecumseh, Okla. (unregulated)
Annual low flow, in cubic feet par

second, for recurrence interval, in $\frac{\text { years, indicated in column subheade }}{2}$

88
96
105
117

291.

$1 \frac{1}{7}$

7
14
30

$\begin{array}{ll}29 & 1.1 \\ 32 & 1.44 \\ 34.6 & 2.08 \\ 44.5 & 3.1\end{array}$

18

22.

22.5
24.6

3.5

4.5

5.8

1.0

1.8

2.2

155

173

193

239

18
77

77
133

238

$\begin{array}{rr}68 & 25 \\ 82 & 30 \\ 96 & 35 \\ 130 & 40\end{array}$

$\begin{array}{rl}4 & 2.5 \\ 13 & 5 \\ 21 & 7\end{array}$

$43 \quad 16$

0
0
0

57.8

.45

.58

.80
1.45

6.2

6.2
6.8

7.5

16

25
30
5
0
2.5
5
7
6

.

1.25
2.80

5.5

9.5

.45

1.49

1.49
2.92

0
0
0

.6

3.5

3.81

4.44

8.61

.4
.6
1.47

1.47
2.8

08

$\begin{array}{r}4 \\ 6 \\ 8 \\ \hline\end{array}$

0

.05

12

.12

.4
.56
.8

.8
2.9

0
0
0
0

.3
.3
.3

.33

.1
.1
.1
.11

.04

.04

.05
.06

1.7

2.5

3.5
5.5

0
0
0
0

0
0
0
0 
Table 2.--Selected low-flow characteristics of streams in Oklahoma.--Continued

\begin{tabular}{|c|c|c|c|c|c|c|}
\hline \multicolumn{2}{|c|}{ Stat1on } & \multirow{2}{*}{$\begin{array}{l}\text { Drainage } \\
\text { area } \\
\left(m 1^{2}\right)\end{array}$} & \multirow{2}{*}{$\begin{array}{l}\text { Duration } \\
\text { In days }\end{array}$} & \multicolumn{3}{|c|}{$\begin{array}{l}\text { Annual low flow, in cubic feet par } \\
\text { second, for recurrence Interval, } \\
\text { years, indicated in column subhee }\end{array}$} \\
\hline Number & Name & & & 2 & 10 & 20 \\
\hline 07230500 & $\begin{array}{l}\text { Little River nr Tecumseh, Okla. } \\
\text { (regulated) }\end{array}$ & 456 & $\begin{array}{r}1 \\
7 \\
14 \\
30\end{array}$ & $\begin{array}{l}\begin{array}{l}0 \\
0 \\
0 \\
\quad .88\end{array}\end{array}$ & $\begin{array}{l}0 \\
0 \\
0 \\
0\end{array}$ & $\begin{array}{l}0 \\
0 \\
0 \\
0\end{array}$ \\
\hline 07230800 & Salt Creek nr Dewr1ght, Okla. & 210 & $\begin{array}{r}1 \\
7 \\
14 \\
30\end{array}$ & $\begin{array}{l}0 \\
1.4 \\
3 \\
5.1\end{array}$ & $\begin{array}{l}0 \\
0 \\
0 \\
0\end{array}$ & $\begin{array}{l}0 \\
0 \\
0 \\
0\end{array}$ \\
\hline 07231000 & $\begin{array}{l}\text { Little R1ver nr Sasakwa, Okla. } \\
\text { (unregulated) }\end{array}$ & 865 & $\begin{array}{r}1 \\
7 \\
14 \\
30\end{array}$ & $\begin{array}{l}3.6 \\
4.33 \\
5.7 \\
10\end{array}$ & $\begin{array}{l}0 \\
.3 \\
.35 \\
.5\end{array}$ & $\begin{array}{l}0 \\
0 \\
0 \\
0\end{array}$ \\
\hline 07231000 & $\begin{array}{l}\text { Little RIver nr Sasakwa, Okla. } \\
\text { (regulated) }\end{array}$ & 865 & $\begin{array}{r}1 \\
7 \\
14 \\
30\end{array}$ & $\begin{array}{l}0 \\
0 \\
.1 \\
.4\end{array}$ & $\begin{array}{l}0 \\
0 \\
0 \\
.02\end{array}$ & $\begin{array}{l}0 \\
0 \\
0 \\
\quad .01\end{array}$ \\
\hline 07231500 & $\begin{array}{l}\text { Canadian River at Calvin, Okla. } \\
\text { (unregulated) }\end{array}$ & 27,952 & $\begin{array}{r}1 \\
7 \\
14 \\
30\end{array}$ & $\begin{array}{l}13.3 \\
20 \\
27 \\
39.9\end{array}$ & $\begin{array}{l}0 \\
0 \\
0 \\
.05\end{array}$ & $\begin{array}{l}0 \\
0 \\
0 \\
0\end{array}$ \\
\hline 07231500 & $\begin{array}{l}\text { Canadian River at Calvin, Okla. } \\
\text { (regulated) }\end{array}$ & 27,952 & $\begin{array}{r}1 \\
7 \\
14 \\
30\end{array}$ & $\begin{array}{r}.9 \\
3.2 \\
9.8 \\
19\end{array}$ & $\begin{array}{l}0 \\
0 \\
.5 \\
2.2\end{array}$ & $\begin{array}{l}0 \\
0 \\
.1 \\
1.3\end{array}$ \\
\hline 07232000 & Gaines Creek nr Krebs, Okla. & 588 & $\begin{array}{r}1 \\
7 \\
14 \\
30\end{array}$ & $\begin{array}{c}0 \\
.1 \\
.4 \\
1.82\end{array}$ & $\begin{array}{l}0 \\
0 \\
0 \\
0\end{array}$ & $\begin{array}{l}0 \\
0 \\
0 \\
0\end{array}$ \\
\hline 07232450 & Tepee Creek nr Eva, Okla. & 95.0 & $\begin{array}{r}1 \\
7 \\
14 \\
30\end{array}$ & $\begin{array}{l}0 \\
0 \\
0 \\
0\end{array}$ & $\begin{array}{l}0 \\
0 \\
0 \\
0\end{array}$ & $\begin{array}{l}0 \\
0 \\
0 \\
0\end{array}$ \\
\hline 07232500 & Beaver River nr Guymon, Okcla. & 2,139 & $\begin{array}{r}1 \\
7 \\
14 \\
30\end{array}$ & $\begin{array}{l}0 \\
.08 \\
.22 \\
.57\end{array}$ & $\begin{array}{l}0 \\
0 \\
0 \\
0\end{array}$ & $\begin{array}{l}0 \\
0 \\
0 \\
0\end{array}$ \\
\hline 07233000 & Coldwater Creek nr Hardesty, Okla. & 1,967 & $\begin{array}{r}1 \\
7 \\
14 \\
30\end{array}$ & $\begin{array}{l}0 \\
0 \\
0 \\
0\end{array}$ & $\begin{array}{l}0 \\
0 \\
0 \\
0\end{array}$ & $\begin{array}{l}0 \\
0 \\
0 \\
0\end{array}$ \\
\hline 07234000 & Beaver River at Beaver, Okla. & 7,955 & $\begin{array}{r}1 \\
7 \\
14 \\
30\end{array}$ & $\begin{array}{l}0 \\
0 \\
0 \\
0\end{array}$ & $\begin{array}{l}0 \\
0 \\
0 \\
0\end{array}$ & $\begin{array}{l}0 \\
0 \\
0 \\
0\end{array}$ \\
\hline 07234100 & Clear Creek nr Blmwood, Okla. & 170 & $\begin{array}{r}1 \\
7 \\
14 \\
30\end{array}$ & $\begin{array}{l}.6 \\
.76 \\
.8 \\
.95\end{array}$ & $\begin{array}{l}.05 \\
.21 \\
.4 \\
.6\end{array}$ & $\begin{array}{l}0 \\
.15 \\
.32 \\
.5\end{array}$ \\
\hline 07234500 & $\begin{array}{l}\text { North Canadian River ar Fort } \\
\text { Supply, Okla. }\end{array}$ & 9,615 & $\begin{array}{r}1 \\
7 \\
14 \\
30\end{array}$ & $\begin{array}{l}0 \\
0 \\
0 \\
0\end{array}$ & $\begin{array}{l}0 \\
0 \\
0 \\
0\end{array}$ & $\begin{array}{l}0 \\
0 \\
0 \\
0\end{array}$ \\
\hline 07235500 & Wolf Creek nr Shattuck, Okla. & 1,183 & $\begin{array}{r}1 \\
7 \\
14 \\
30\end{array}$ & $\begin{array}{l}0 \\
0 \\
0 \\
.75\end{array}$ & $\begin{array}{l}0 \\
0 \\
0 \\
0\end{array}$ & $\begin{array}{l}0 \\
0 \\
0 \\
0\end{array}$ \\
\hline 07236000 & Wolf Creek ar Fargo, Okla. & 1,624 & $\begin{array}{r}1 \\
7 \\
14 \\
30\end{array}$ & $\begin{array}{r}.6 \\
.9 \\
1.5 \\
3.8\end{array}$ & $\begin{array}{l}0 \\
0 \\
0 \\
0\end{array}$ & $\begin{array}{l}0 \\
0 \\
0 \\
0\end{array}$ \\
\hline
\end{tabular}


Table 2.--Selected low-flow characteristics of streams in Oklahoma.--Continued

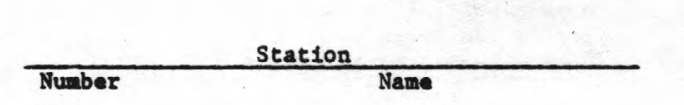

Drafnage
area

$\left(\mathrm{mi}^{2}\right)$

Duration in days

07237000 Wolf Creak nr Fort Supply, Okla. 1,739

07237500 North Canadian River at Woodward, 11,589 Okla. (unregulated)

07237500

North Canadian RIver at Woodward, Okla. (regulated)

07238000

North Canadian RIver nr Sealing,

12,261 Okla.

07239000

North Canadian River at Canton,

Okla. (unregulated)

12,484

07239000 North Canadian River at Canton, Okla. (regulated)

12,484

07239500

North Canadian River nr El Reno, Okla. (unregulated)

13,042

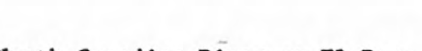

07239500 North Canadian River nr El Reno, Okla. (regulated)

13,042

Toreh changan River ar oklahoma

$07241500 \quad$ North Canadian River nr OkIahoma City, Okla.

13,354$$
\text { (a) }
$$
07241500 North Canadian River nr Harrah, Okla. (unregulated)

13,501

(1)

07242000 North Canadian River nr Wetumka, Okla.

14,290

$07242500 \quad$ Bellcow Creek at Chandler, Okla.

$07243000 \quad$ Dry Creek nr Kendrick. Okla.



07243500 Deep Fork nr Beggs, Okla.

2,018

07244000

Deep Fork nr Dewar, Okla.

1
7
14
30

1

7
14
30

Annual low flow, in cublc feet per

second, for recurrence interval, in

$\frac{\text { years, indicated in column subheads }}{2}$

$\begin{array}{lll}.21 & 0 & 0 \\ .34 & 0 & 0 \\ .42 & 0 & 0 \\ .62 & .03 & 0\end{array}$

0

2.5

2.5

0
0

.01

.3

0

.3
.6
.8

$\begin{array}{ll}0 & 0 \\ 0 & 0 \\ 0 & 0 \\ 0 & 0\end{array}$

rar

0

0

0
0
0


Table 2.--Selected low-flow characteristics of streams in Oklahoma.--Continued

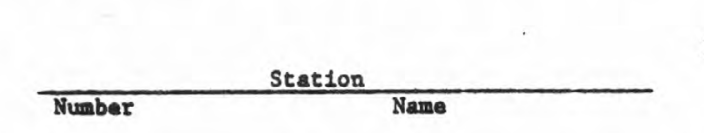
Drainage $\left(m i^{2}\right)$

07245000 Canadian River nr Whitefield, Okla. 47,576 (unregulated)

07245000 Canadian River nr Whitefield, Okla. 47,576 (regulated)

07245500

Sallisaw Creek nr Sallisaw, Okla.

182

$\begin{array}{r}07246500 \\ \hline\end{array}$

Arkansas River nr Sallisaw, Okla. 147,757 (unregulated)

07246500 Arkansas River nr Sallisaw, Okla. 147,757

07247500 Fourche Maline nr Red Oak, Okla.

07248500 Poteau River nr Wister, Okla. (unregulated) (regulated)

0724900 Poteau River nr Poteau, Okla.

07249800 Lee Creek ar Short, Okla.

236

$07249900 \times$ Little Lee Creek ar Short, Okla.

103

07250000 Lee Creek nr Van Buren, Ałk.

426

07300500

Salt Fork Red River at Mangum, Okla.

1,566

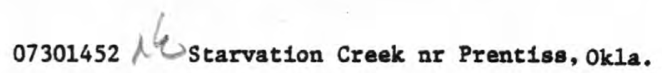

44.3

07301460 Turkey Creek nr Sayre, Okla.

49.5
Annual low flow, in cublc feet per second, for recurrence interval, in $\frac{\text { years, 1ndicated in column subheads }}{2}$ Durat1

$\begin{array}{lr}198 & 4 \\ 211 & 10 \\ 232 & 17 \\ 370 & 29\end{array}$

60
240

325

$\begin{array}{lr}1.1 & .5 \\ 1.3 & .6 \\ 2.2 & 1.1 \\ 2.5 & 1.3\end{array}$

.78

1.05

1.45

2.5

1
2
4
9
.5
.6
1.1
1.3

1
7
14
30

2.6

0

.1

0
0
0
0

$\begin{array}{lrr}3,150 & 400 & 130 \\ 4,500 & 500 & 200 \\ 5,280 & 580 & 280 \\ 6,100 & 700 & 390 \\ & & \\ 2,550 & 900 & 600 \\ 4,100 & 1,150 & 800 \\ 5,600 & 1,360 & 950 \\ 7,000 & 1,600 & 1,100\end{array}$

0

.01

.3800

0

0.03
.09

.03
.09
.55

1.5

4.4

6.6
7.8

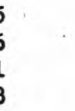

.5

1.3

2.6

.16

.29

1.0

0

.023

.15

$1_{3}^{.8}$

1
7
14
30

0
0
0
0

0

0

.12
.17

.60
.62
.71
.80
.05 
Table 2.--Selected low-flow characteristics of streams in Oklahoma.--Continued

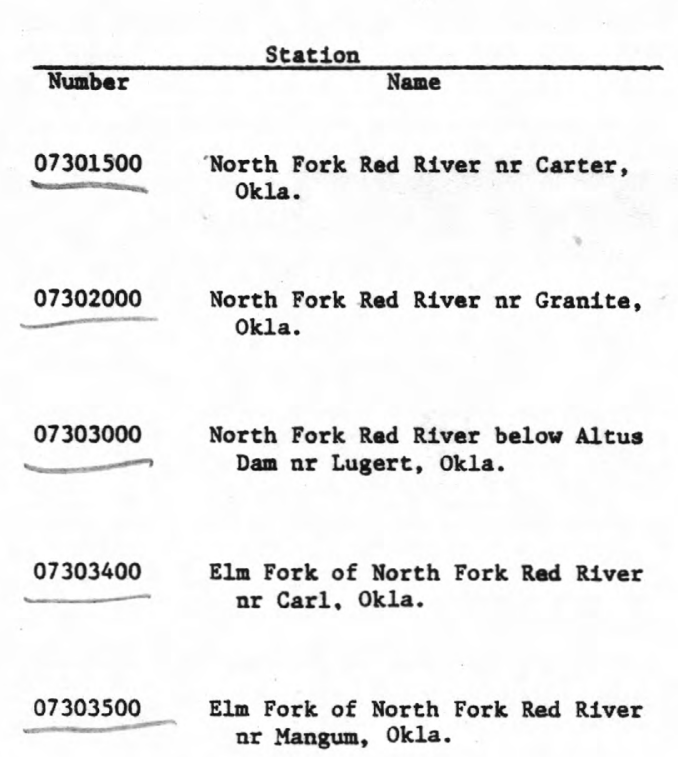

Drainage

area

2,337

2,494

1
7
14
30

2,515

416

838

nr Mangum, Okla.

07304500 Elk Creek nr Hobart, Okla.

549

07305000

North Fork Red River nr Headrick, Okla. (unregulated)

4,244

07305000

North Fork Red River nr Headrick, Okla. (regulated)

07305500 West Otter Creek at Snyder Lake nr Mountain Park, Okla.

132

4,244

244

Duration

Annual low flow, in cublc feet per

second, for recurrence interval, in years, indicated in column subheads

In days

$\begin{array}{lll}2 & 10 & 2 \\ 0 & 0 & 0 \\ 0 & 0 & 0 \\ 0 & 0 & 0 \\ 0 & 0 & 0 \\ 0 & 0 & 0 \\ 0 & 0 & 0 \\ 0 & 0 & 0 \\ 0 & 0 & 0 \\ 0 & 0 & 0 \\ 0 & 0 & 0 \\ 0 & 0 & 0 \\ 0 & 0 & 0\end{array}$

1.4

2.6

3.1

.04
.07
.1
.5

1.2

1.8

2.2

3.7

.3
.4
.6

13.3
16.7

25
57

$\begin{array}{rrr}.2 & 0 & 0 \\ .7 & 0 & 0 \\ 1.7 & 0 & 0 \\ 4.8 & 0 & 0\end{array}$

07306500 Otter Creek at Mountain Park, Okla.

164

07308310 N Suttle Creek nr Davidson, Okla.

55.0

$07310000 / / 6$ Little Medicine Bluff Creek nr Lawton, Okla.

7.0

07310500 Medicine Bluff Creek nr Lawton,

101

$$
\text { okla. }
$$

675

07311200 Blue Beaver Creek nr Cache, Okla.

$$
\begin{aligned}
& 0 \\
& 0 \\
& 0 \\
& 0
\end{aligned}
$$

$\begin{array}{ll}0 & 0 \\ 0 & 0 \\ 0 & 0 \\ 0 & 0\end{array}$

07311000 East Cache Creek nr Walters, Okla.

24.6 
Table 2.--Selected low-flow characteristics of streams in Oklahoma.--Continued

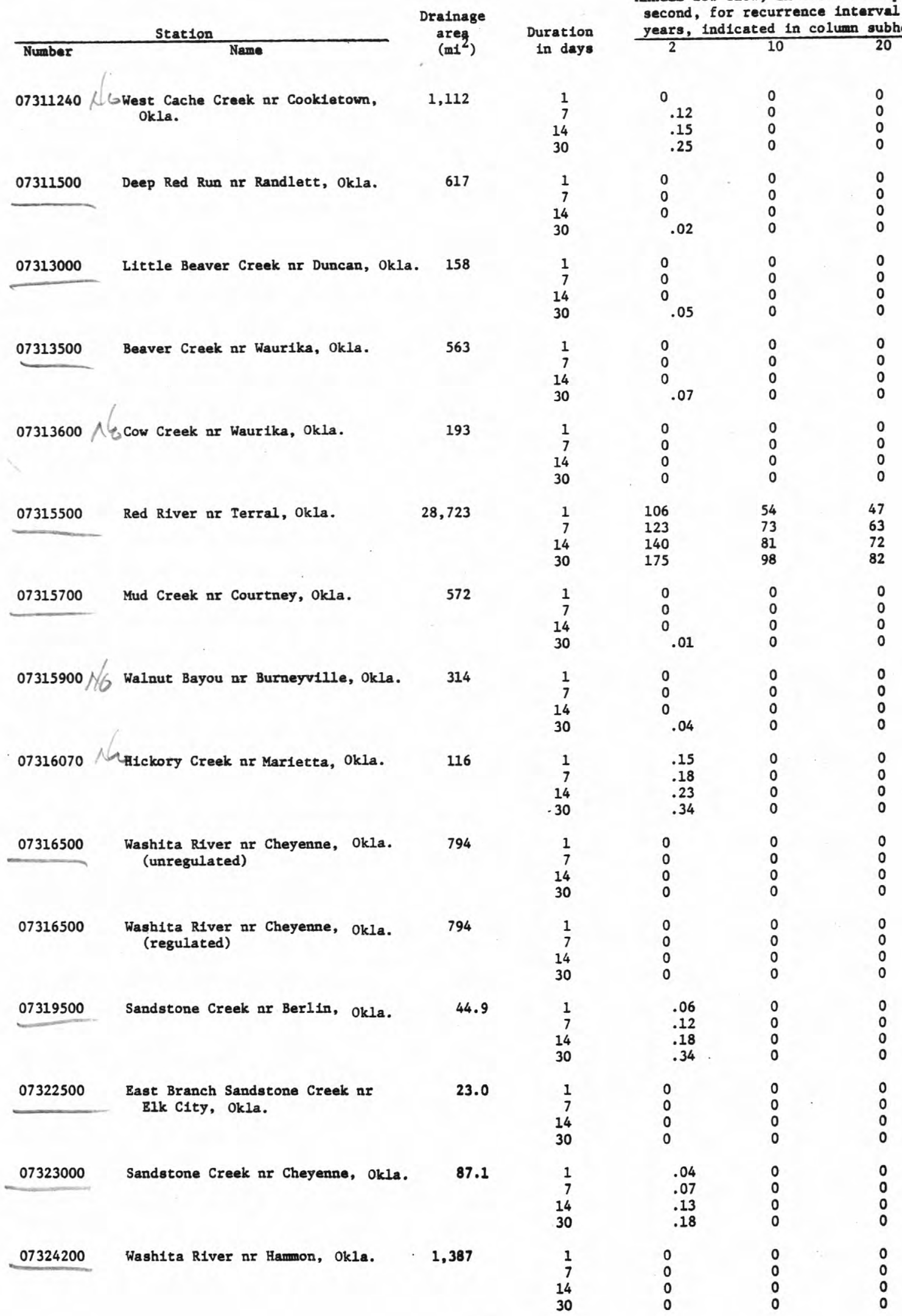


Table 2.--Selected low-flow characteristics of streams in Oklahoma.--Continued

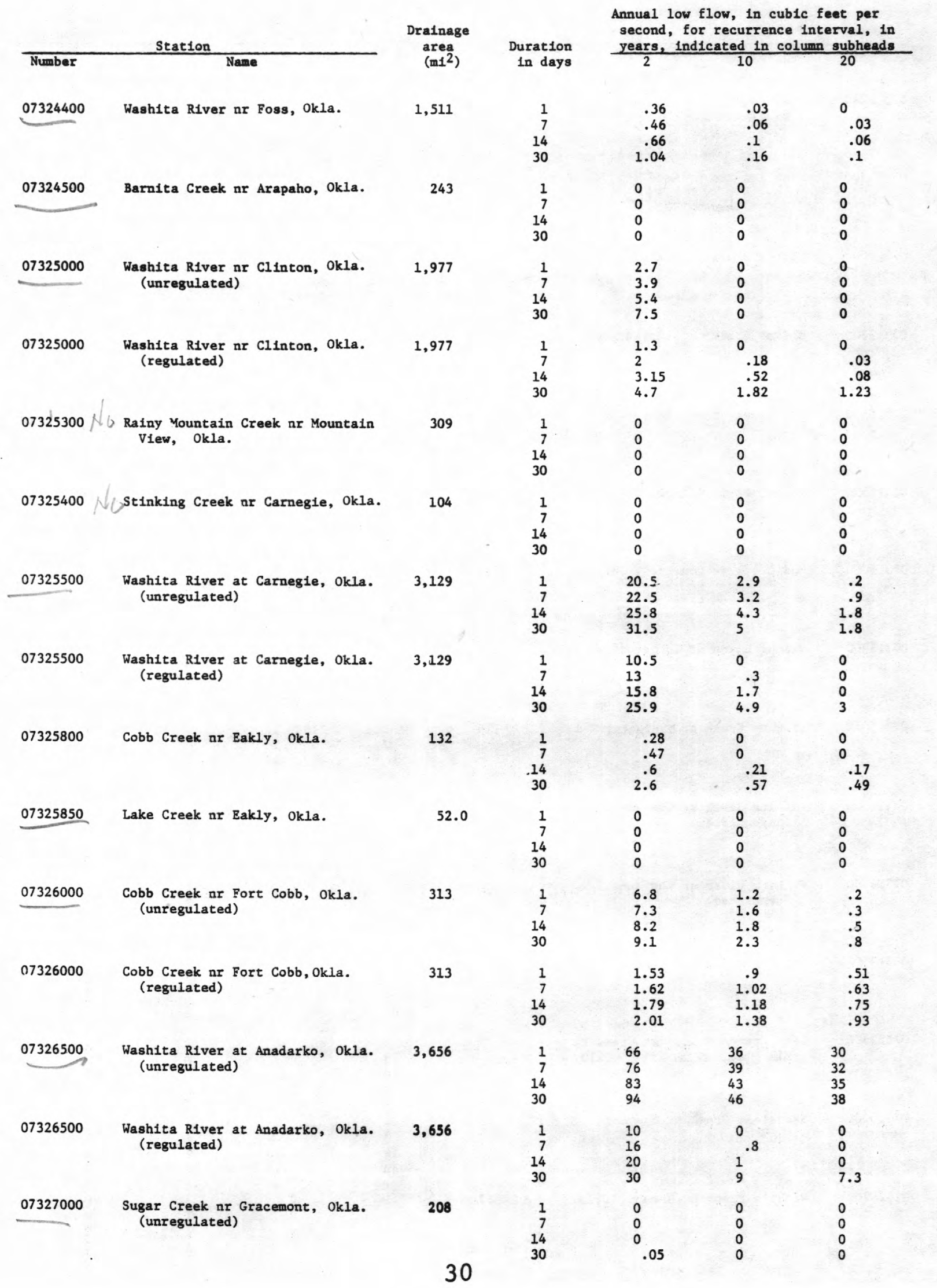


Table 2.--Selected low-flow characteristics of streams in Oklahoma.--Continued

\begin{tabular}{|c|c|c|c|c|c|c|}
\hline \multicolumn{2}{|r|}{ Station } & \multirow{2}{*}{$\begin{array}{c}\text { Drainage } \\
\text { area } \\
\left(m 1^{2}\right)\end{array}$} & \multirow{2}{*}{$\begin{array}{l}\text { Duration } \\
\text { in days }\end{array}$} & \multicolumn{3}{|c|}{$\begin{array}{l}\text { second, for recurrence interval, in } \\
\text { years, indicated in column subheade }\end{array}$} \\
\hline Number & Name & & & 2 & 10 & 20 \\
\hline 07327000 & $\begin{array}{l}\text { Sugar Creek nr Gracemont, Okla. } \\
\text { (regulated) }\end{array}$ & 208 & $\begin{array}{r}1 \\
7 \\
14 \\
30\end{array}$ & $\begin{array}{l}0 \\
.025 \\
.05 \\
.1\end{array}$ & $\begin{array}{l}0 \\
0 \\
0 \\
0\end{array}$ & $\begin{array}{l}0 \\
0 \\
0 \\
0\end{array}$ \\
\hline 07327490 & $\begin{array}{l}\text { Little Washita River nr Ninnekah, } \\
\text { Okla. }\end{array}$ & 208 & $\begin{array}{r}1 \\
7 \\
14 \\
30\end{array}$ & $\begin{array}{l}0 \\
0 \\
0 \\
.53\end{array}$ & $\begin{array}{l}0 \\
0 \\
0 \\
0\end{array}$ & $\begin{array}{l}0 \\
0 \\
0 \\
0\end{array}$ \\
\hline 07327500 & $\begin{array}{l}\text { Little Washita River at Ninnekah, } \\
\text { Okla. }\end{array}$ & 227 & $\begin{array}{r}1 \\
7 \\
14 \\
30\end{array}$ & $\begin{array}{l}2.5 \\
3.8 \\
4.3 \\
5.9\end{array}$ & $\begin{array}{l}0 \\
0 \\
0 \\
0\end{array}$ & $\begin{array}{l}0 \\
0 \\
0 \\
0\end{array}$ \\
\hline 07328000 & Washita River nr Tabler, Okla. & 4,706 & $\begin{array}{r}1 \\
7 \\
14 \\
30\end{array}$ & $\begin{array}{r}65 \\
92 \\
108 \\
133\end{array}$ & $\begin{array}{l}27 \\
31.5 \\
33.5 \\
38\end{array}$ & $\begin{array}{l}24 \\
26 \\
27 \\
31\end{array}$ \\
\hline 07328070 & Winter Creek nr Alex, Okla. & 33 & $\begin{array}{r}1 \\
7 \\
14 \\
30\end{array}$ & $\begin{array}{l}0 \\
.15 \\
.24 \\
.54\end{array}$ & $\begin{array}{l}0 \\
0 \\
0 \\
\quad .02\end{array}$ & $\begin{array}{l}0 \\
0 \\
0 \\
0\end{array}$ \\
\hline 07328300 & Finn Creek ar Story, Okla. & 67.2 & $\begin{array}{r}1 \\
7 \\
14 \\
30\end{array}$ & $\begin{array}{r}.09 \\
.40 \\
.60 \\
1.05\end{array}$ & $\begin{array}{r}0 \\
0 \\
-0 \\
0\end{array}$ & $\begin{array}{l}0 \\
0 \\
0 \\
0\end{array}$ \\
\hline 07328500 & $\begin{array}{l}\text { Washita River nr Pauls Valley, } \\
\text { Ok1a. }\end{array}$ & 5,330 & $\begin{array}{r}1 \\
7 \\
14 \\
30\end{array}$ & $\begin{array}{r}63 \\
75 \\
84 \\
102\end{array}$ & $\begin{array}{l}0 \\
0 \\
0 \\
3\end{array}$ & $\begin{array}{l}0 \\
0 \\
0 \\
0\end{array}$ \\
\hline 07329000 & Rush Creek at Purdy, Okla. & 145 & $\begin{array}{r}1 \\
7 \\
14 \\
30\end{array}$ & $\begin{array}{l}2.3 \\
3.1 \\
4 \\
6.6\end{array}$ & $\begin{array}{l}0 \\
0 \\
.8 \\
.35\end{array}$ & $\begin{array}{l}0 \\
0 \\
0 \\
0\end{array}$ \\
\hline 07329500 & $\begin{array}{l}\text { Rush Creek nr Maysville, Okla. } \\
\text { (unregulated) }\end{array}$ & 206 & $\begin{array}{r}1 \\
7 \\
\cdot 14 \\
30\end{array}$ & $\begin{array}{r}0 \\
.1 \\
.2 \\
1.4\end{array}$ & $\begin{array}{l}0 \\
0 \\
0 \\
0\end{array}$ & $\begin{array}{l}0 \\
0 \\
0 \\
0\end{array}$ \\
\hline 07329500 & $\begin{array}{l}\text { Rush Creek nr Maysville, Okla. } \\
\text { (regulated) }\end{array}$ & 206 & $\begin{array}{r}1 \\
7 \\
14 \\
30\end{array}$ & $\begin{array}{l}0 \\
0 \\
0 \\
0\end{array}$ & $\begin{array}{l}0 \\
0 \\
0 \\
0\end{array}$ & $\begin{array}{l}0 \\
0 \\
0 \\
0\end{array}$ \\
\hline 07329700 & Wildhorse Creek nr Hoover, Okla. & 604 & $\begin{array}{r}1 \\
7 \\
14 \\
30\end{array}$ & $\begin{array}{l}0 \\
0 \\
.05 \\
.6\end{array}$ & $\begin{array}{l}0 \\
0 \\
0 \\
0\end{array}$ & $\begin{array}{l}0 \\
0 \\
0 \\
0\end{array}$ \\
\hline 07329900 & Rock Creek at Dougherty & 138 & $\begin{array}{r}1 \\
7 \\
14 \\
30\end{array}$ & $\begin{array}{l}2.6 \\
4 \\
5.2 \\
6.3\end{array}$ & $\begin{array}{r}.4 \\
.8 \\
1.3\end{array}$ & $\begin{array}{l}.2 \\
.4 \\
.6 \\
.8\end{array}$ \\
\hline 07330500 & Caddo Creek nr Ardmore, Okla. & 298 & $\begin{array}{r}1 \\
7 \\
14 \\
30\end{array}$ & $\begin{array}{l}0 \\
0 \\
0 \\
.21\end{array}$ & $\begin{array}{l}0 \\
0 \\
0 \\
0\end{array}$ & $\begin{array}{l}0 \\
0 \\
0 \\
0\end{array}$ \\
\hline 07331000 & Washita River nr Durwood, Olkla. & 7,202 & $\begin{array}{r}1 \\
7 \\
14 \\
30\end{array}$ & $\begin{array}{l}100 \\
126 \\
138 \\
171\end{array}$ & $\begin{array}{r}6 \\
9 \\
12 \\
15\end{array}$ & $\begin{array}{l}4 \\
6 \\
7 \\
9\end{array}$ \\
\hline
\end{tabular}


Table 2.--Selected low-flow characteristics of streams in Oklahoma.--Continued

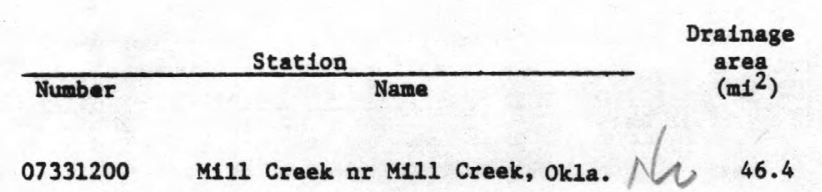

$07331300 \quad$ Pennington Creek nr Reagon, okla. $/ / 6$

65.7

$07331600 \times 6$ Red River at Denison Dam nr Denison, Tex. 07332000 Red River nr Colbert, Okla.

07332000

Red RIver nr Colbert, Okla. (regulated)

07332250 (CIsland Bayou nr Albany, Okla.

07332400 Blue Creek at M1lburn, Okla.

$07332500 \times(6$ Blue River nr Blue, Okla.

476

$07332700 \times$ CMuddy Boggy Creek nr Parker, Okla.

174

$07333500 \quad$ Chickasaw Creek nr Stringtown, Okla.

32.7

07333800

McGee Creek nr Stringtown, Okla.

86.6

0733400 Muddy Boggy Creek nr Farris, Okla. 1,087

$07334200 / 6$ Byrds Mill spring nI Fittstown,

$07334400 / 6$ Clear Boggy Creek nr Tupelo, Okla.

248

$07334420 \mathrm{~N}($ Leader Creek at Tupelo, Okla.

132

203

64.3

Annual low flow, in cublc feet per second, for recurrence interval, in $\frac{\text { years, Indicated in column subheads }}{20}$ Duration
in days

\section{2}

$$
2.9
$$

3.3

1.68
1.2
1.4

1.4

.44
.68
.86

7.0

9.6

9.6
13.0

13.5

2.4

3.3
3.9

4.8

60

220

220
520

900

$\begin{array}{rr}50 & 40 \\ 70 & 60 \\ 150 & 100 \\ 420 & 300 \\ 72 & 45 \\ 125 & 82 \\ 150 & 105 \\ 200 & 140\end{array}$

380

450

485

535

1.1

100
400

760

1,120

.03

.03
.05

.08

40

40
45
50

50
80

$\begin{array}{lll}33 & 18 & 16 \\ 35 & 19 & 17 \\ 39 & 22 & 18 \\ 44 & 25 & 20\end{array}$

23
25
27

27.8

2.5

7

13.9

1.5

2.3

1.0

1.3

40

100

5

105

140

20

28

45

0

.10
.17

.17
.40

0

0
0
0

0

0

0

0

$\begin{array}{rl}2 & 0 \\ 4 & 0 \\ 8 & 0 \\ 21 & 0\end{array}$

$\bar{z}$

7.0
7.8

8.8

11.0

$\begin{array}{lll}0 & 0 & 0 \\ 0 & 0 & 0 \\ 0 & 0 & 0 \\ 0 & 0 & 0\end{array}$


Table 2.--Selected low-flow characteristics of streams in Oklahoma.--Continued

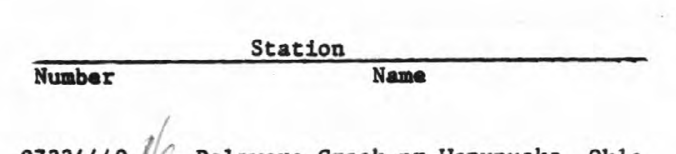

$07334440 /(C$ Delaware Creek nr Wapunucka, Okla.

Drainage $\underset{\left(m i^{2}\right)}{\operatorname{area}}$

45.8

07335000

Clear Boggy Creek nr Caney, Okla.

$07335500 \times /$ Red River at Arthur C1ty, Tex:

44,531

07335700

Kiamich1 River nr B1g Cedar, Okla.

40.1

$07335900 N$ Buck Creek nr Moyers, Okla.

100

68.0

07336000 Tenmile Creek nr Miller, Okla.

720

$1 \quad 670$

1,050

1,350

1,800

1

7
14

30
Annual low flow, in cublc feet per second, for recurrence interval, in years, indicated in column subheads 2010

.18
.24
.42
.75

9

11.6

11.6

$\begin{array}{ll}0 & 0 \\ 0 & 0 \\ 0 & 0 \\ 0 & 0\end{array}$

$\begin{array}{ll}.1 & 0 \\ .3 & 0 \\ .5 & .1 \\ .8 & .4\end{array}$

.1

$\begin{array}{ll}230 & 150 \\ 300 & 180 \\ 380 & 190 \\ 630 & 300\end{array}$

50

300
$07336500 \quad \begin{aligned} & \text { Kiamichi River nr Belzoni, Okla. } \\ & 07337500 \quad \begin{array}{c}\text { Little River nr Wright City, Okla. } \\ \text { (unregulated) }\end{array} \\ & \begin{array}{c}\text { Litele River nr Wright City, Okla. } \\ \text { (regulated) }\end{array}\end{aligned}$

07337900 Glover Creek nr Glover, Okla.

315

1,423

645

645

$07338000 / 0$ Little River nr Idabel, 0kla.

1,173

$07338500 /$ Little River below Lukfata Creek

1,226 nr Idabel, Okla. (unregulated)

07338500

Little RIver below Lukfata Creek nr Idabel, Okla. (regulated)

07339000

Mountain Fork nr Eagleton, Ok1s. (unregulated)

1,226

787
.06

.21

.42

0

.12

.45

0
0
0
0

1.7

3.3

4.4

5.3

1.5

1.8

3.5

0
0
0
0

$$
\begin{aligned}
& 0 \\
& 0 \\
& 0 \\
& 0
\end{aligned}
$$

2.1

3.5

6.8

13.1

$\begin{array}{ll}0 & 0 \\ 0 & 0 \\ 0 & 0 \\ 0 & 0\end{array}$

.1

.0
1.7

2
2.8
3.9

3.9
5.2

12

13.8

16
26

19

27.5

39

2.8
3.9
5

10 
Täble 2.--Selected low-flow characterističs of streams in Oklahoma.--Continued

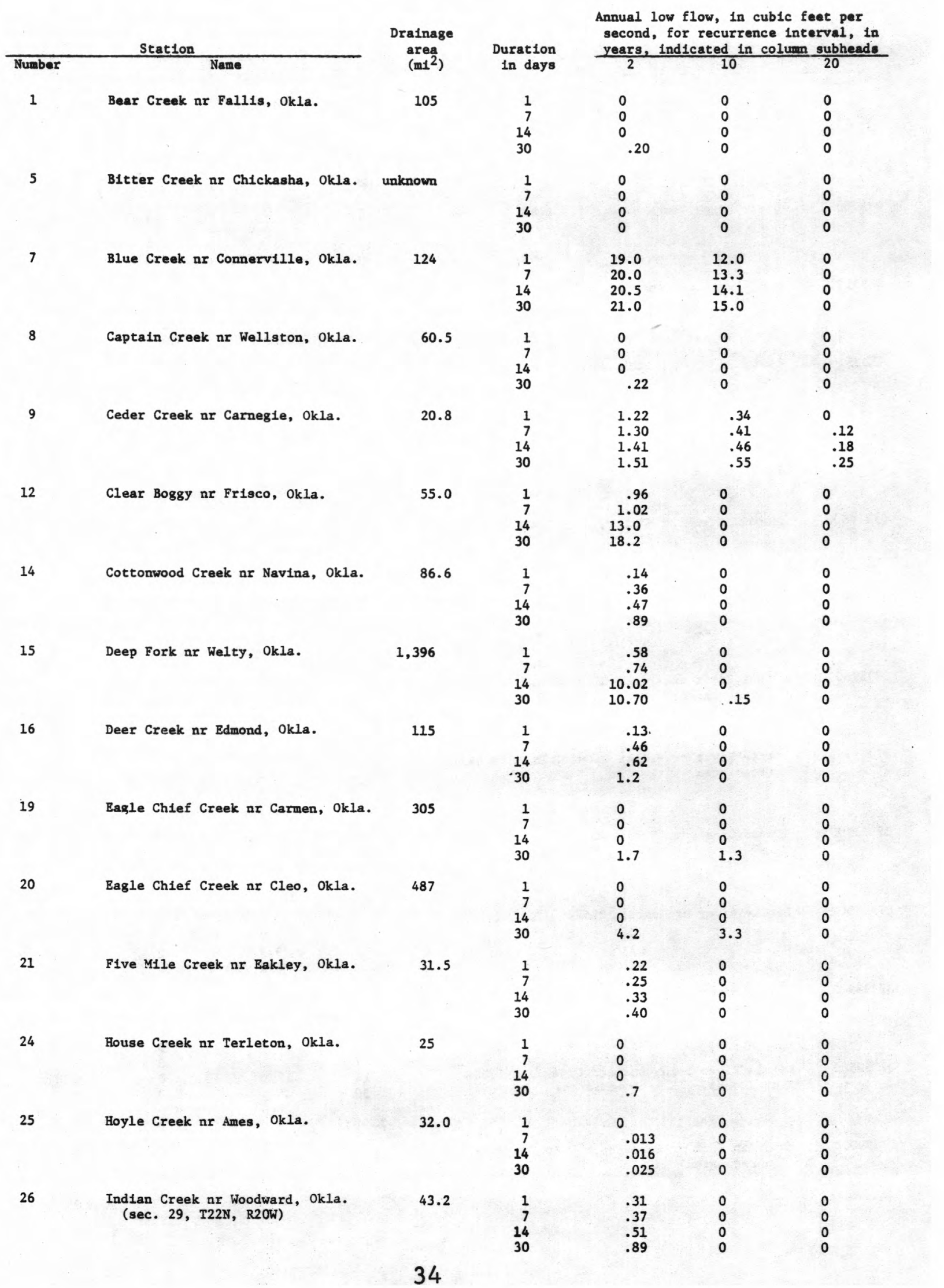


Table 2.--Selected low-flow characteristics of streams in Oklahoma.--Continued

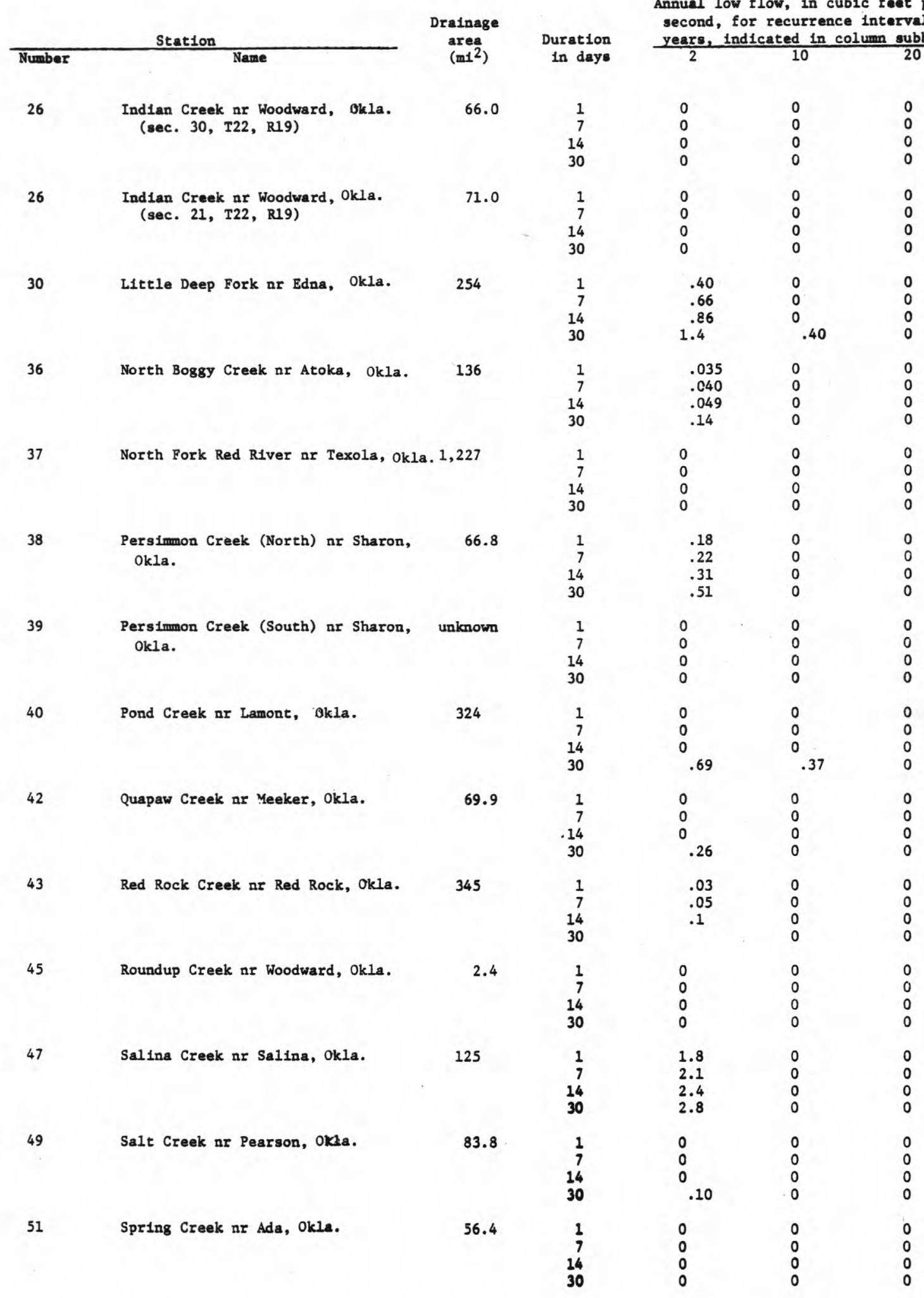


Table 2.--Selected low-flow characteristics of streams in Oklahoma.--Continued

$\frac{\text { Station }}{\text { Number }}$

52 Sweetwater Creek nr Texola, Okla.

54 Turkey Creek ar Dover, Okla.

55

West Bitter Creek nr Tabler, Okla.

56

Wewoka Creek nr Wetumka, Okla.

$\begin{array}{cc}\text { Drainage } \\ \text { area } \\ \left(m i^{2}\right) & \begin{array}{r}\text { Duration } \\ \text { In days }\end{array}\end{array}$

436

106

396

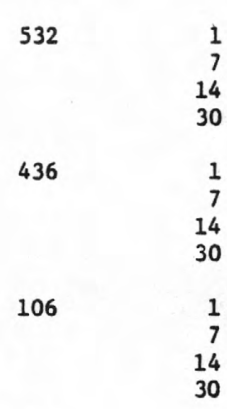

$$
\begin{array}{r}
1 \\
7 \\
14 \\
30 \\
\\
1 \\
7 \\
14 \\
30
\end{array}
$$

Annual low flow, in cublc feet per second, for recurrence interval, in years, indlcated in column subheads

$\begin{array}{lll}.98 & 0 & 0 \\ 1.48 & 0 & 0 \\ 2.2 & 0 & 0 \\ 4.0 & 0 & 0 \\ 0 & 0 & 0 \\ 0 & 0 & 0 \\ 0 & 0 & 0 \\ 2.0 & 1.5 & 0\end{array}$

0
0
0
0
0
0
0
0
0
0
0
0

.02

.03

.05

20

3.3

4.8

6.8

10.5

0
0
0
0
0
0
0
0


An analysis of error was done for each regional polygon to obtain general information and not specific errors at each site. Continuous-record sites other than the index station were correlated with the index station for randomly selected low-flow periods as if only partial record existed. The 7-day, 2-year and 30-day, 20-year low flows obtained from the correlation procedure were compared to the frequencies computed from the station record. Error estimates resulting from this procedure ranged from $0.97 \mathrm{log}$ units to $0.13 \mathrm{log}$ units. Polygons 1 and 3 had only one useable continuous record station, and low flows in polygon 10 were all zero and no error analysis was determined. The estimated errors in the 7-day, 2-year and the 30-day, 20-year lows were averaged and shown in table 3. The low-flow frequencies computed from station record are assumed to be the exact low flows for the station, which does not consider the time-sampling error of the station record. However, the time-sampling error is small in comparison to the correlation analysis error so the values in table 3 are good indicators of the accuracy that can be expected.

Table 3.--Expected error in low-flow estimates for regional polygon.

\begin{tabular}{lccc}
\hline $\begin{array}{l}\text { Regional } \\
\text { polygon }\end{array}$ & $\begin{array}{l}\text { Average error } \\
\text { in log units }\end{array}$ & $\begin{array}{l}\text { Regional } \\
\text { polygon }\end{array}$ & $\begin{array}{l}\text { Average error } \\
\text { in log units }\end{array}$ \\
\hline & No data & 7 & 0.91 \\
1 & 0.73 & 8 & .83 \\
2 & No data & 9 & .50 \\
3 & .49 & 10 & Zero low flows \\
4 & .19 & 11 & .97 \\
5 & .52 & 12 & .79 \\
6 & & 13 & .13 \\
\hline
\end{tabular}

Conclusions and Recommendations for Further Study

This study has generated some useful information for those who are concerned with low flows of streams in 0klahoma, and the study results show some areas where further investigation would be benericial. Study results indicate natural basin streams with drainage areas of less than $1,000 \mathrm{mi}^{2}$ are dry at least for a short time during the year. Exceptions are streams in the northeastern part of the State and areas in southcentral Oklahoma. Small rural uncontrolled basins in areas outside northeast and south-central Oklahoma flow only during storm runoff events and no further low-flow study is warranted.

Additional study of low flows in the northeast and south-central areas as well as studies of large rivers and controlled basins are necessary. More concentrated effort should be placed on analysis of existing data and on obtaining additional information on areas of appreciable low flow. The data collection should be more complete and the analysis should involve all streams of significant size.

Index stations should be as much a part of the low-flow data collection plan as the low-flow measurement sites. Continuous recording stations should be established specifically as index stations on streams which are similar in character to those in which correlations are to be made if errors of estimate are to be kept within reasonable limits. 
Additional low-flow information could be obtained if a study of the low-flow characteristics of short reaches of large rivers were undertaken. One continuous-record site analyzed individually may be of marginal use because it is a composite of so many factors within the large basin. However, if a portion of the river were analyzed between continuous-record sites, the results would reflect characteristics of only that portion of the basin between record sites. This analysis would be of particular benefit because there are over 50 gaging sites with extensive record on large rivers in the State which would add significantly to the low-flow information if analyzed in short reaches. Analysis of stream reaches also solves the problem of control structures on large rivers as uncontrolled reaches may be analyzed separately from controlled reaches.

The effect of flow retention and other manmade structures on low flows also should be investigated, and seepage and stored storm runoff released on a continuous basis through release structures may result in a pronounced change in low flows downstream. Sustained low flows resulting from urban, industrial and agricultural return flows may show either beneficial or harmful effects downstream.

Water quality at low flows also is an important aspect of streamflow. The quality of the sustained flow in a natural stream determines man's activities for the present and the future. Addition of chemical and biological constituents in a stream may create uninhabitable conditions downstream or elimination of constituents may cause undesirable erosion and degradation of the stream channel. A study of water quality should be the next step in analyzing low-flows.

\section{SELECTED REFERENCES}

Mize, L.D., 1975, Statistical summaries of streamflow records, Oklahoma through 1974: U.S. Geol. Survey open-file report, 399 p.

Riggs, H.C., 1968, Some statistical tools in hydrology: U.S. Geol. Survey Techniques Water-Resources Inv., Chap. Al, Book 4, 39 p.

Riggs, H.C., 1972, Low-flow investigations: U.S. Geol. Survey Techniques Water-Resources Inv., Chap. B1, Book 4, 18 p.

U.S. Geological Survey, 1948-1975, Water resources data for Oklahoma: U.S. Geol. Survey Water-Data Reports, 1948-1975.

U.S. Geologica1 Survey, 1972, Index for Surface-Water Records to September 30, 1970, Part 7: U.S. Geol. Survey Circ. 657, 67 p. 
APPENDIX I

MISCELLANEOUS AND PARTIAL-RECORD LOW-FLOW MEASUREMENT DATA 
Antelope Springs near Sulphur, Oklahoma, Miscellaneous site.

Lat. $34^{\circ} 00^{\circ} 00^{\prime \prime}$, long. $96^{\circ} 56^{\circ} 00^{\prime \prime}, \mathrm{NW} 1 / 4 \mathrm{NE} 1 / 4 \mathrm{sec} .1, \mathrm{~T} .1 \mathrm{~S}, \mathrm{R}^{3} \mathrm{E}$, in Platt National Park, $2 \mathrm{miles}$ east of Sulphur, Okla.

Date Discharge

$11-15 \cdot 55$

0

Aurora Creek near Beaver, Oklahoma, Miscellaneous site.

Lat. $36^{\circ} 50^{\prime} 00^{\prime \prime}$, long. $100^{\circ} 20^{\prime} 00^{\circ}, \mathrm{SW}^{\prime} 1 / 4 \mathrm{SE} 1 / 4 \mathrm{sec} .6, \mathrm{~T} .4 \mathrm{~N}$., R.24 E., at bridge on U.S. Highway 270 1 mile north of Beaver, Okla.

Date Discharge

$10-23-51 \quad 6.56$

Bear Creek near Fallis, Oklahoma, Miscellaneous site.

Lat. $35^{\circ} 46^{\prime} 0 \mathrm{C}^{\prime \prime}$, long $97^{\circ} 07^{\prime} 31^{\prime \prime}$, NE $1 / 4 \mathrm{sec} .31$, T.is N., R.2 E., at bridge on county road, $1 / 2 \mathrm{mile}$ southwest of Fállis, okla.

Date Discharge Date Discharge Date Discharge Date Discharge

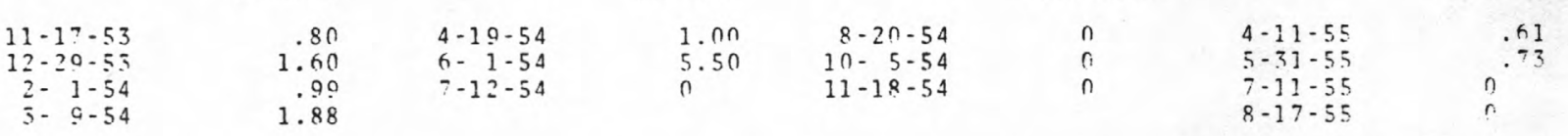

Beaver (Sand) Creek near Texhoma, Oklahoma, 07232400 , partial record site.

lat. $36^{\circ} 36^{\prime} 08^{\prime \prime}$, long. $101^{\circ} 47^{\prime} 50^{\prime \prime}$, on east line, séc.31, T.2 N., R.12 E., Texas County, at bridge on State Highwav $95,6.5$ miles north of Texhoma.

\begin{tabular}{|c|c|c|c|c|c|c|c|}
\hline Date & $\begin{array}{c}\text { Discharge } \\
\left(\mathrm{ft}^{3} / \mathrm{s}\right)\end{array}$ & Date & $\begin{array}{c}\text { Discharge } \\
\left(\mathrm{ft}^{3} / \mathrm{s}\right)\end{array}$ & Date & $\begin{array}{c}\text { Discharge } \\
\left(\mathrm{ft}^{3} / \mathrm{s}\right)\end{array}$ & Date & $\begin{array}{c}\text { Discharge } \\
\left(\mathrm{ft}^{3} / \mathrm{s}\right)\end{array}$ \\
\hline $\begin{array}{r}11-23-66 \\
1-17-67 \\
4-24-67 \\
8-7-67 \\
10-16-67\end{array}$ & $\begin{array}{l}0 \\
0 \\
0 \\
0 \\
0\end{array}$ & $\begin{array}{r}2-26-68 \\
5-20-68 \\
8-26-68 \\
12-24-68 \\
3-28-69\end{array}$ & $\begin{array}{l}0 \\
0 \\
0 \\
0 \\
0\end{array}$ & $\begin{array}{r}5-27-69 \\
8-21-69 \\
12-3-69 \\
3-10-70 \\
5-19-70\end{array}$ & $\begin{array}{l}0 \\
0 \\
0 \\
0 \\
0\end{array}$ & $\begin{array}{r}8-7-70 \\
12-2-71 \\
3-5-71 \\
6-15-71 \\
9-21-71\end{array}$ & $\begin{array}{l}\hat{0} \\
\hat{0} \\
\hat{0} \\
0\end{array}$ \\
\hline
\end{tabular}

Beaver Creek near Kak City, Oklahoma, Miscellaneous site.
Lat. $36^{\circ} 48^{\prime} 00^{\circ}$, long $96^{\circ} 49^{\circ} 00^{\prime \prime}$, SE $1 / 4 \mathrm{SH} 1 / 4 \mathrm{sec} .24$, T.27 N., R.4 E., at county highway bridge, 2 miles north of Kar City, Okla.

Date

$\begin{array}{cc}\begin{array}{c}\text { Discharge } \\ \left(\mathrm{ft}^{3} / \mathrm{s}\right)\end{array} & \text { Date } \\ 0 & 4-26-54 \\ 0 & 5-11-54 \\ 0 & 6-2-54 \\ 0 & 7-6-54 \\ 0.01 & 8-2-54 \\ 0 & 9-9-54\end{array}$

Discharge
$\left(\mathrm{ft}^{3} / \mathrm{s}\right)$
2.09
6.96
13.8
0
0
0

Date

Discharge
$\left(\mathrm{ft} \mathrm{t}^{3} / \mathrm{s}\right)$

Date

Discharge

$11-10-53$

$12-16-53$

$1-12-54$

$2-16-54$

$3-1-54$

4- 5-54

$9-9-54$

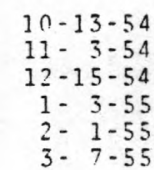

$4-5-55$

5- 3- 55

6- $-28-55$

$7-13-55$

8- $1-55$

$\mathrm{ft} / \mathrm{s}$

beaver Creek near Waurika, Oklahoma, Miscellaneous site

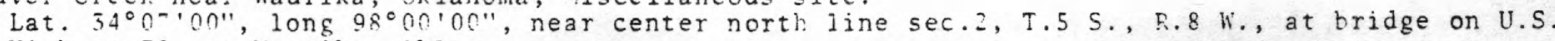

Highwar 70, at haurika, Okila.

$\begin{array}{ccccccc}\text { Date } & \begin{array}{c}\text { Discharge } \\ \left(\mathrm{ft}^{3} / \mathrm{s}\right)\end{array} & \text { Date } & \begin{array}{c}\text { Discharge } \\ \left(\mathrm{ft}^{3} / \mathrm{s}\right)\end{array} & \text { Date } & \begin{array}{c}\text { Discharge } \\ \left(\mathrm{ft}^{3} / \mathrm{s}^{2}\right.\end{array} & \begin{array}{c}\text { Date } \\ \left(\mathrm{ft} / \mathrm{s}^{2}\right)\end{array} \\ 8-20-49 & 0 & 8-16-49 & 0 & 10-31-50 & 40.7 & 10-29-59\end{array}$


Bent Creek near Richmond, Oklahoma, 0723780 , partial record site.

Lat. $36^{\circ} 11^{\prime}$, long. $99^{\circ} 02^{\prime}$, SE lit SE i/t sec.21, T.20 K., R.li in., imiles east of Richmond, Okla.

\begin{tabular}{|c|c|c|c|c|c|c|c|}
\hline Date & $\begin{array}{c}\text { Discharge } \\
\left(\mathrm{ft}^{3} / \mathrm{s}\right)\end{array}$ & Date & $\begin{array}{c}\text { Discharge } \\
\left(\mathrm{ft}^{3} / \mathrm{s}\right)\end{array}$ & Date & $\begin{array}{c}\text { Discharge } \\
\left(\mathrm{ft}^{3} / \mathrm{s}\right)\end{array}$ & Date & $\begin{array}{c}\text { Discharge } \\
\left(\mathrm{ft}^{3} / \mathrm{s}\right)\end{array}$ \\
\hline $\begin{array}{l}2-20-44 \\
9-28-48 \\
3-16-56 \\
4-18-56\end{array}$ & $\begin{array}{r}2.03 \\
1.51 \\
1.19 \\
.74\end{array}$ & $\begin{array}{l}5-22-56 \\
6-18-56 \\
2-26-57\end{array}$ & $\begin{array}{l}0 \\
0 \\
.94\end{array}$ & $\begin{array}{l}4-12-5 ? \\
7-11-5 ? \\
8-14-5 ?\end{array}$ & $\begin{array}{r}1.62 \\
3.64 \\
.13\end{array}$ & $\begin{array}{r}9-11-57 \\
10-17-57 \\
11-12-57 \\
12-20-57\end{array}$ & $\begin{array}{l}.11 \\
1.32 \\
1.45 \\
1.83\end{array}$ \\
\hline
\end{tabular}

Bent Creek near Seiling, Oklahomá, 07237800 , partial record site.

Lat $36^{\circ} 11^{\prime} 26^{\prime \prime}$, long. $90^{\circ} 00^{\prime} 36^{\prime \prime}$, in $\mathrm{NK} 1 / 4 \mathrm{SE} 1 / 4 \mathrm{sec} .21$, T.20 N., R.17 W., woodward County, at bridge on U.S. Highways 183 and 270,6 miles northwest of Seiling, and at mile 2.0 .

\begin{tabular}{|c|c|c|c|c|c|c|c|}
\hline Date & $\begin{array}{c}\text { Discharge } \\
\left(\mathrm{ft}^{3} / \mathrm{s}\right)\end{array}$ & Date & $\begin{array}{c}\text { Discharge } \\
\left(\mathrm{ft}^{3} / \mathrm{s}\right)\end{array}$ & Date & $\begin{array}{c}\text { Discharge } \\
\left(\mathrm{ft}^{3} / \mathrm{s}\right)\end{array}$ & Date & $\begin{array}{c}\text { Discharge } \\
\left(\mathrm{ft}^{3} / \mathrm{s}\right)\end{array}$ \\
\hline $\begin{array}{r}11-5-70 \\
3-4-71 \\
5-27-71\end{array}$ & $\begin{array}{l}.63 \\
1.5 \\
.77\end{array}$ & $\begin{array}{c}0-8-71 \\
12-16-71\end{array}$ & 0 & $\begin{array}{l}2-17-72 \\
5-25-72\end{array}$ & $\begin{array}{l}1.7 \\
1.1\end{array}$ & $\begin{array}{r}8-15-72 \\
12-21-72 \\
3-2-73\end{array}$ & $\begin{array}{l}1.3 \\
3.1 \\
5.5\end{array}$ \\
\hline
\end{tabular}

Big Canyon Springs near Fittstown, Oklahoma, Miscellaneous site.

Lat. $34^{\circ} 322^{\circ}$, long. $96^{\circ} 37^{\prime}$, SE $1 / 4 \mathrm{SE} 1 / 4 \mathrm{sec} .10$, T.1 N., R. 7 E., at site $5-3 / 4$ miles southeast of Fittstown, Dkla.

$\begin{array}{lrrr}\text { Date } & \begin{array}{r}\text { Discharge } \\ \left(\mathrm{ft}^{3} / \mathrm{s}\right)\end{array} & \text { Date } & \begin{array}{r}\text { Discharge } \\ \left(\mathrm{ft}^{3} / \mathrm{s}\right)\end{array} \\ 8-17-54 & 1.06 & 12-22-54 & 1.04\end{array}$

Bitter Creek near Chickasha, Ck1 ahoma, Miscellaneous site.

Lat. $35^{\circ} 00^{\prime}$, long. $97^{\circ} 51^{\prime}$, NW $1 / 4 \mathrm{sec} .16$, T. 6 . N., R. 6 W., at county highway bridge, $3 / 4 \mathrm{mile}$ south of Lucille and 6 miles southeast of Chickasha, okla.

\begin{tabular}{|c|c|c|c|c|c|c|c|}
\hline Date & $\begin{array}{c}\text { Discharge } \\
\left(\mathrm{ft}^{3} / \mathrm{s}\right)\end{array}$ & Date & $\begin{array}{c}\text { Discharge } \\
\left(\mathrm{ft}^{3} / \mathrm{s}\right)\end{array}$ & Date & $\begin{array}{c}\text { Discharge } \\
\left(\mathrm{ft}^{3} / \mathrm{s}\right)\end{array}$ & Date & $\begin{array}{c}\text { Discharge } \\
\left(\mathrm{ft}^{3} / \mathrm{s}\right)\end{array}$ \\
\hline $\begin{array}{r}11-50-51 \\
1-16-52\end{array}$ & $\begin{array}{l}8.74 \\
1.39\end{array}$ & $3-6-52$ & 4.22 & $4-25-52$ & 6.05 & $6-3-52$ & 15.3 \\
\hline
\end{tabular}

Bitter Creek near Hitchcock, Oklahoma, Miscellaneous site.

Lat. $36^{\circ} 02: 00^{\prime \prime}$, long. $96^{\circ} 32^{\prime} 46^{\prime \prime}$, NE $1 / 4 \mathrm{SW} 1 / 4$ sec.28, T.18 N., R.11 W., at county highway bridge, 3 miles northwest of Hitchcock, Okla.

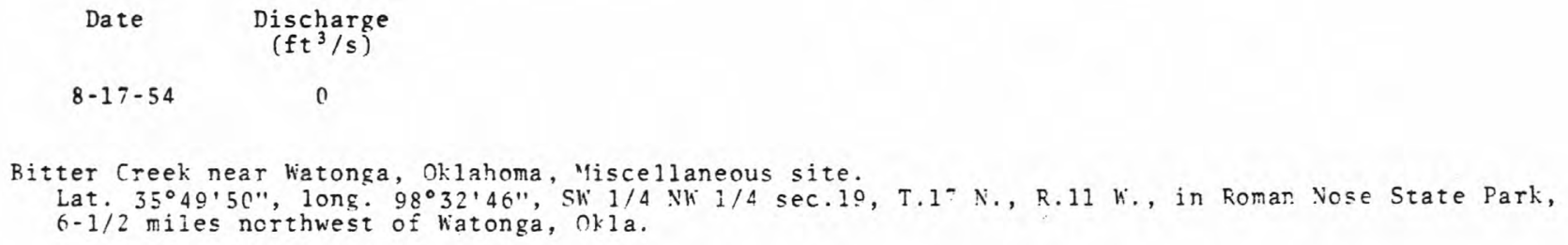


Black Bear Creek near Pawnee, Oklahoma, Miscellaneous site.

Lat. $63^{\circ} 20^{\prime} 18^{\prime \prime}$, long. $96^{\circ} 32^{\prime} 46^{\prime \prime}$, NE $1 / 4 \mathrm{SE} 1 / 4 \mathrm{sec} .31$, T.22 N., R.6. E., at county highway bridge, $1 / 2$ mile downstream from Camp Creek and 6 miles east of Pawnee, Okla.

$\begin{array}{cr}\text { Date } & \begin{array}{c}\text { Discharge } \\ \left(\mathrm{ft}^{3} / \mathrm{s}\right)\end{array} \\ 10-24.52 & \end{array}$

Black Fork near Page, Oklahoma, Miscellaneous site.

Lat. $34^{\circ} 52^{\prime} 30^{\prime \prime}$, long. $94^{\circ} 30^{\prime}$, in sec. 5 , T. $13 \mathrm{~N}$., R. $27 \mathrm{E}$. , at highway bridge, 2.0 miles west of Arkansas-Oklahoma State line, 5.0 miles northeast of Page, Okla.

$\begin{array}{rrrrrrr}\text { Date } & \begin{array}{c}\text { Discharge } \\ \left(\mathrm{ft}^{3} / \mathrm{s}\right)\end{array} & \text { Date } & \begin{array}{c}\text { Discharge } \\ \left(\mathrm{ft}^{3} / \mathrm{s}\right)\end{array} & \text { Date } & \begin{array}{c}\text { Discharge } \\ \left(\mathrm{ft}^{3} / \mathrm{s}\right)\end{array} & \begin{array}{c}\text { Date } \\ \left(\mathrm{ft}^{3} / \mathrm{s}^{2}\right)\end{array} \\ 8-7-58 & 1.87 & 11-6-58 & .05 & 8-18-59 & 0 & 8-11-60 \\ 10-8-58 & .11 & 7-2-59 & .35 & 7-20-60 & 14.9 & 0-14-60\end{array}$
Blue Creek near Connerville, Oklahoma, Miscellaneous site,
Lat. $34^{\circ} 24^{\prime} 30^{\prime \prime}$, long. $96^{\circ} 38^{\circ}$, SW $1 / 4 \mathrm{sec} .24, \mathrm{~T} .1 \mathrm{~S} ., \mathrm{R} .6 \mathrm{E}$, at bridge on brate Highway $99,1 / 4 \mathrm{mile}$
north of Connerville, Okla.

\begin{tabular}{|c|c|c|c|c|c|c|c|}
\hline Date & $\begin{array}{c}\text { Discharge } \\
\left(\mathrm{ft}^{3} / \mathrm{s}\right)\end{array}$ & Date & $\begin{array}{c}\text { Discharge } \\
\left(\mathrm{ft}^{3} / \mathrm{s}\right)\end{array}$ & Date & $\begin{array}{c}\text { Discharge } \\
\left(\mathrm{ft}^{3} / \mathrm{s}\right)\end{array}$ & Date & $\begin{array}{c}\text { Discharge } \\
\left(\mathrm{ft}^{3} / \mathrm{s}\right)\end{array}$ \\
\hline $\begin{array}{r}9-10-51 \\
11-27-51 \\
1-16-52 \\
3-5-52 \\
4-23-52 \\
6-11552 \\
7-22-52 \\
9-3-52 \\
10-9-52 \\
11-18-52 \\
12-23-52\end{array}$ & $\begin{array}{r}36.3 \\
25.9 \\
19.7 \\
23.8 \\
160 . \\
37.5 \\
21.6 \\
19.3 \\
18.9 \\
18.0 \\
16.4\end{array}$ & $\begin{array}{r}2-4-53 \\
3-10-53 \\
4-14-53 \\
6-2-53 \\
6-23-53 \\
8-19-53 \\
9-30-53 \\
11-17-53 \\
1-12-54 \\
2-25-54 \\
4-20-54\end{array}$ & $\begin{array}{l}15.0 \\
18.6 \\
25.6 \\
37.6 \\
29.7 \\
34.6 \\
24.4 \\
22.7 \\
23.2 \\
22.7 \\
16.0\end{array}$ & $\begin{array}{r}5-28-54 \\
7-14-54 \\
8-24-54 \\
9-16-54 \\
11-23-54 \\
12-21-54 \\
2-1-55 \\
3-2-55 \\
6-13-55 \\
7-27-55 \\
9-21-55\end{array}$ & $\begin{array}{l}67.9 \\
28.9 \\
23.4 \\
20.7 \\
17.9 \\
22.1 \\
28.0 \\
26.6 \\
25.6 \\
17.4 \\
15.1\end{array}$ & $\begin{array}{r}10-31-55 \\
1-4-56 \\
2-14-56 \\
3-29-56 \\
5-21-56 \\
6-19-56 \\
7-18-56 \\
8-21-56 \\
8-28-56 \\
9-24-56 \\
11-27-56\end{array}$ & $\begin{array}{l}20.2 \\
15.3 \\
18.0 \\
15.4 \\
14.2 \\
20.1 \\
14.2 \\
14.7 \\
13.2 \\
11.8 \\
11.2\end{array}$ \\
\hline
\end{tabular}

Blue Creek at Milburn, Oklahoma, 07332400 , partial record site.

Lat $34^{\circ} 15^{\prime}$, long. $97^{\circ} 30^{\prime}$, SW $1 / 4 \mathrm{SW}^{\prime} 1 / 4 \mathrm{sec} .35$, T. $3 \mathrm{~S}$., R. $7 \mathrm{E}$., on downstream side of left bank pier of bridge on State Highway $48 \mathrm{~A}, 1 / 2 \mathrm{mile}$ north of Milburn.

\begin{tabular}{|c|c|c|c|c|c|c|c|}
\hline Date & $\begin{array}{c}\text { Discharge } \\
\left(\mathrm{ft}^{3} / \mathrm{s}\right)\end{array}$ & Date & $\begin{array}{c}\text { Discharge } \\
\left(\mathrm{ft}^{3} / \mathrm{s}\right)\end{array}$ & Date & $\begin{array}{c}\text { Discharge } \\
\left(\mathrm{ft}^{3} / \mathrm{s}\right)\end{array}$ & Date & $\begin{array}{c}\text { Discharge } \\
\left(\mathrm{ft}^{3} / \mathrm{s}\right)\end{array}$ \\
\hline $\begin{array}{r}8-28-56 \\
9-24-56 \\
11-27-56 \\
1-21-57 \\
3-13-57 \\
7-31-57\end{array}$ & $\begin{array}{l}13.6 \\
14.9 \\
19.1 \\
25.6 \\
46.7 \\
92.0\end{array}$ & $\begin{array}{r}11-12-57 \\
1-17-58 \\
3-19-58 \\
6-11-58 \\
9-5-58 \\
11-5-58\end{array}$ & $\begin{array}{r}262.0 \\
170.0 \\
114.0 \\
120.0 \\
54.3 \\
33.8\end{array}$ & $\begin{array}{r}1-8-59 \\
6-9-59 \\
9-15-59 \\
10-27-59 \\
12-8-59 \\
3-23-60\end{array}$ & $\begin{array}{l}40.4 \\
15.7 \\
33.2 \\
51.4 \\
37.3 \\
82.8\end{array}$ & $\begin{array}{r}8-25-60 \\
12-1-60 \\
1-12-61 \\
3-1-61 \\
5-17-61 \\
7-19-61\end{array}$ & $\begin{array}{r}71.2 \\
67.1 \\
124.0 \\
74.0 \\
59.4 \\
50.0\end{array}$ \\
\hline
\end{tabular}

Boggy Creek near Bessie, Oklahoma, Miscellaneous site.

Lat. $35^{\circ} 22^{\prime} 09^{\prime \prime}$, long. $98^{\circ} 58^{\prime} 30^{\prime \prime}, \mathrm{SW} 1 / 4 \mathrm{sec} .2, \mathrm{~T} .10 \mathrm{~N} ., \mathrm{R} .17 \mathrm{~W}$., at bridge on U.S. Highway 183 , $1-1 / 2$ miles southeast of Bessie, Okla.

$\begin{array}{cc}\text { Date } & \begin{array}{c}\text { Discharge } \\ \left(\mathrm{ft}^{3} / \mathrm{s}\right)\end{array} \\ 8-27-52 & 0\end{array}$


Boggy Creek near Cordell, Oklahoma, Miscellaneous site.

Lat. $35^{\circ} 18^{\prime} 55^{\prime \prime}$, long. $98^{\circ} 54^{\prime} 51^{\prime \prime}$, S line sec.27, T.10 N., R.16 W., at county highway bridge 6 miles east of Cordell, Okla.

Date $\begin{gathered}\text { Discharge } \\ \left(\mathrm{ft}^{3} / \mathrm{s}\right)\end{gathered}$

Boggy Creek near Lamont, Oklahoma, Miscellaneous site.

Lat. $36^{\circ} 40^{\prime} 37^{\prime \prime}$, long. $97^{\circ} 26^{\prime} 17^{\prime \prime}$, north line sec.32, T.26 N., R.3 W., at bridge on U.S. Highway 60 , $3 / 4$ mile east of Lamont, Oklahoma.

Date

$$
\begin{aligned}
& \text { Discharge } \\
& \left(\mathrm{ft}^{3} / \mathrm{s}\right)
\end{aligned}
$$

$10-27-47$

$$
.04
$$

Bois d'Arc Creek near Ponca City, Oklahoma, 07152250 , partial record site.

Lat. $36^{\circ} 40^{\prime} 00^{\prime \prime}$, long. $97^{\circ} 07^{\prime} 30^{\prime \prime}$, on south line SW 1/4 SE $1 / 4$ sec.6, T.25 N., R.2 E., Kay County, at county road bridge 1 mile south and 0.5 mile east of junction of U.S. Highways $6 n$ and $77,5 \mathrm{miles}$

\begin{tabular}{|c|c|c|c|c|c|c|c|}
\hline Date & $\begin{array}{c}\text { Discharge } \\
\left(\mathrm{ft}^{3} / \mathrm{s}\right)\end{array}$ & Date & $\begin{array}{c}\text { Discharge } \\
\left(\mathrm{ft}^{3} / \mathrm{s}\right)\end{array}$ & Date & $\begin{array}{c}\text { Discharge } \\
\left(\mathrm{ft}^{3} / \mathrm{s}\right)\end{array}$ & Date & $\begin{array}{c}\text { Discharge } \\
\left(\mathrm{ft}^{3} / \mathrm{s}\right)\end{array}$ \\
\hline $\begin{array}{r}12-3-52 \\
1-13-65 \\
7-16-65 \\
11-3-65 \\
3-9-66 \\
6-1-66 \\
11-22-66 \\
3-\quad 1-67\end{array}$ & $\begin{array}{c}.95 \\
10.4 \\
3.08 \\
.57 \\
2.5 \\
.31 \\
.31 \\
3.0\end{array}$ & $\begin{array}{r}8-9-67 \\
9-29-67 \\
1-21-68 \\
4-16-68 \\
7-30-68 \\
10-9-68 \\
2-11-69\end{array}$ & $\begin{array}{l}3.9 \\
4.7 \\
4.5 \\
4.9 \\
1.6 \\
3.8 \\
4.4\end{array}$ & $\begin{array}{r}5-21-69 \\
8-1-69 \\
12-22-69 \\
2-17-70 \\
5-12-70 \\
6-29-70 \\
8-12-70\end{array}$ & $\begin{array}{l}5.5 \\
3.2 \\
6.8 \\
8.4 \\
6.5 \\
2.3 \\
.05\end{array}$ & $\begin{array}{r}11-12-70 \\
3-23-71 \\
6-24-71 \\
8-19-71 \\
11-11-71 \\
3-7-72 \\
4-18-72 \\
7-11-72\end{array}$ & $\begin{array}{l}.87 \\
2.8 \\
2.8 \\
.05 \\
3.3 \\
4.3 \\
5.6 \\
.20\end{array}$ \\
\hline
\end{tabular}
southwest of Ponca City and about 4 miles upstream from mouth.

Brush Creek near Wyandotte, Oklahoma, Miscellaneous site.

Lat. $36^{\circ} 47^{\prime} 04^{\prime \prime}$, long. $94^{\circ} 40^{\prime} 34^{\prime \prime}$, SE $1 / 4 \mathrm{sec} .35$, T.27 N., R. $24 \mathrm{E}$, at county highway bridge, 3 miles southeast of Wyandotte, Okla.

Date Discharge

$12-21-53 \quad 3.38$

Buck Creek near Moyers, Oklahoma, 07335900 , partial record site.

Lat $34^{\prime \prime 2} 20^{\prime} 22^{\prime \prime}$, long. $95^{\circ} 35^{\prime} 55^{\prime \prime}$, in NE $1 / 4$ sec.32, T.2 S., R.16 E., Pushmataha County, at bridge on

\begin{tabular}{|c|c|c|c|c|c|c|c|}
\hline Date & $\begin{array}{c}\text { Discharge } \\
\left(\mathrm{ft}^{3} / \mathrm{s}\right)\end{array}$ & Date & $\begin{array}{c}\text { Discharge } \\
\left(\mathrm{ft}^{3} / \mathrm{s}\right)\end{array}$ & Date & $\begin{array}{c}\text { Dis =harge } \\
\left(\mathrm{ft}^{3} / \mathrm{s}\right)\end{array}$ & Date & $\begin{array}{c}\text { Discharge } \\
\left(\mathrm{ft}^{3} / \mathrm{s}\right)\end{array}$ \\
\hline $\begin{array}{r}11-4-55 \\
12-1-55 \\
1-5-56 \\
2-14-56 \\
4-11-56 \\
5-22-56 \\
7-18-56 \\
8-21-56 \\
10-1-56 \\
12-4-56 \\
1-21-57 \\
7-30-57 \\
10-3-57 \\
2-19-59 \\
7-8-59 \\
7-15-59 \\
8-26-59\end{array}$ & $\begin{array}{c}.46 \\
1.39 \\
.69 \\
83.0 \\
10.3 \\
2.99 \\
.23 \\
2.70 \\
0 \\
.83 \\
5.56 \\
.84 \\
26.6 \\
8.84 \\
6.04 \\
1.03 \\
.53\end{array}$ & $\begin{array}{r}10-15-59 \\
13-27-59 \\
12-8-59 \\
1-26-60 \\
3-22-60 \\
11-30-60 \\
2-28-61 \\
5-16-61 \\
11-1-61 \\
2-13-62 \\
5-17-62 \\
7-10-62 \\
8-14-62 \\
12-19-62 \\
5-21-63 \\
8-20-63\end{array}$ & $\begin{array}{c}1.38 \\
7.72 \\
5.01 \\
62.5 \\
44.4 \\
2.16 \\
100 . \\
17.4 \\
6.76 \\
35.9 \\
7.56 \\
.79 \\
.06 \\
14.6 \\
2.92 \\
0\end{array}$ & $\begin{array}{r}10-11-63 \\
12-3-63 \\
2-18-64 \\
9-16-64 \\
12-15-64 \\
3-10-65 \\
6-17-65 \\
12-22-65 \\
3-15-66 \\
4-7-66 \\
6-8-66 \\
8-31-66 \\
2-16-67 \\
5-25-67 \\
8-30-67 \\
12-7-67\end{array}$ & $\begin{array}{l}0 \\
0 \\
6.85 \\
3.86 \\
51.6 \\
45.1 \\
18.2 \\
1.6 \\
36.0 \\
3.8 \\
.58 \\
4.8 \\
3.6 \\
25.0 \\
0 \\
5.2\end{array}$ & $\begin{array}{r}2-15-68 \\
9-13-68 \\
12-11-68 \\
5-13-69 \\
8-13-69 \\
12-9-69 \\
12-18-69 \\
4-15-70 \\
8-12-70 \\
3-4-71 \\
6-22-71 \\
7-28-71 \\
8-31-71 \\
3-28-72 \\
6-12-72 \\
8-23-72\end{array}$ & $\begin{array}{c}47.0 \\
.03 \\
36.0 \\
83.0 \\
.31 \\
5.7 \\
9.9 \\
26.0 \\
0 \\
64.0 \\
1.8 \\
5.9 \\
.18 \\
.63 \\
0 \\
0\end{array}$ \\
\hline
\end{tabular}
State Highway 144,1 mile northeast of Moyers. 
Buffalo Creek near Edith, Oklahoma, Miscellaneous site.

Lat. $36^{\circ} 46^{\prime 377^{\prime \prime}}$ long. $99^{\circ} 17^{\prime} 04^{\prime \prime}$, $\mathrm{SW} 1 / 4 \mathrm{sec} .36, \mathrm{~T} .27 \mathrm{~N} .$, R. $20 \mathrm{~W}$., at bridge on county road $5-1 / 2$ miles west of Edith, Okla.

Date

$$
\begin{aligned}
& \text { Discharge } \\
& \left(\mathrm{ft}^{3} / \mathrm{s}\right)
\end{aligned}
$$

$11-11-43$

3.02

Buffalo Springs near Sulfur, Oklahoma, Miscellaneous site.

Lat. 34. $30^{\circ} 00^{\prime \prime}$, long. $96^{\circ} 57^{\prime} 43^{\prime \prime}$, SE $1 / 4 \mathrm{NW} 1 / 4 \mathrm{NE} 1 / 4 \mathrm{sec} .1, \mathrm{~T} .1 \mathrm{~S} ., \mathrm{R} .3 \mathrm{E}$. , in Platt National Park, 2 miles east of Sulfur, Okla.

$$
\text { Date Discharge }
$$

$11-15-55$

$$
0
$$

Byrd's Mill Spring near Ada, Oklahoma, Miscellaneous site.

\begin{tabular}{|c|c|c|c|c|c|c|c|}
\hline Date & $\begin{array}{c}\text { Discharge } \\
\left(\mathrm{ft}^{3} / \mathrm{s}\right)\end{array}$ & Date & $\begin{array}{c}\text { Nischarge } \\
\left(\mathrm{ft}^{3} / \mathrm{s}\right)\end{array}$ & Date & $\begin{array}{c}\text { Discharge } \\
\left(\mathrm{ft}^{3} / \mathrm{s}\right)\end{array}$ & Date & $\begin{array}{c}\text { Discharge } \\
\left(\mathrm{ft}^{3} / \mathrm{s}\right)\end{array}$ \\
\hline $\begin{array}{l}2-5-52 \\
3-18-53 \\
9-30-53 \\
4-20-54\end{array}$ & $\begin{array}{l}11.9 \\
10.5 \\
16.0 \\
11.1\end{array}$ & $\begin{array}{r}8-17-54 \\
12-22-54 \\
11-29-55 \\
4-10-56\end{array}$ & $\begin{array}{c}17.1 \\
11.3 \\
10.3 \\
7.76\end{array}$ & $\begin{array}{r}12-17-56 \\
4-9-57 \\
7-9-57 \\
12-13-57\end{array}$ & $\begin{array}{l}5.60 \\
11.3 \\
33.1 \\
27.1\end{array}$ & $\begin{array}{l}1-28-59 \\
8-27-59 \\
9-14-59\end{array}$ & $\begin{array}{l}4.07 \\
2.42 \\
1.41\end{array}$ \\
\hline
\end{tabular}
Lat. $34^{\circ} 36^{\prime}$, long. $96^{\circ} 40^{\circ}, \mathrm{SW} 1 / 4 \mathrm{sec} .34, \mathrm{~T} .2 \mathrm{~N} ., \mathrm{R} .6 \mathrm{E} ., 2$ miles southwest of Fittstown and $12 \mathrm{miles}$ south of Ada, Ok1a.

Cache Creek near Medicine Park, Oklahoma, Miscellaneous site.

Lat. $34^{\circ} 43^{\prime} 23^{\prime \prime}$, long. $98^{\circ} 24^{\prime} 34^{\prime \prime}$, SE $1 / 4 \mathrm{sec} .18$, T.3 N., R.11 w., at bridge on county road, $2 \mathrm{miles}$ south of Richards Spur and 6 miles east of Medicine Park, Okla.

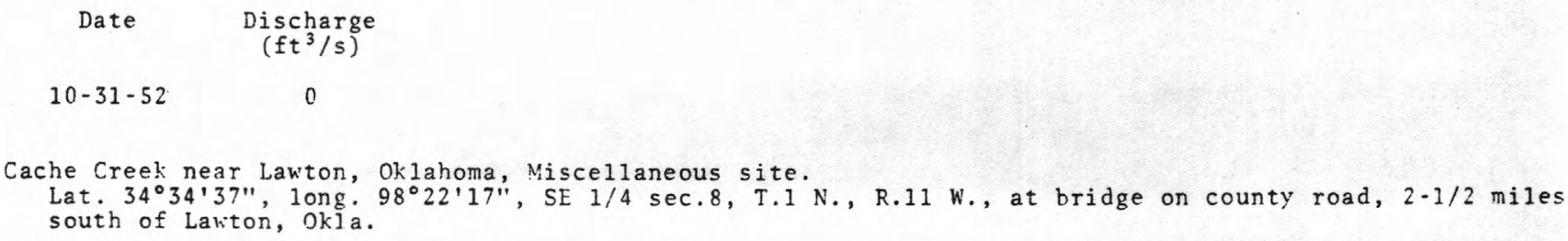

Caney Creek near Caney, Oklahoma, Miscellaneous site. Lat. $34^{\circ} 14^{\prime} 46^{\prime \prime}$, long. $96^{\circ} 12^{\prime} 34^{\prime \prime}$, in SW $1 / 4$ sec.36, T.3 S., R.10 E., at site 300 ft above mouth and 1 mile north of Caney.

$\begin{array}{lccccr}\text { Date } & \begin{array}{c}\text { Discharge } \\ \left(\mathrm{ft}^{3} / \mathrm{s}\right)\end{array} & \text { Date } & \begin{array}{c}\text { Discharge } \\ \left(\mathrm{ft}^{3} / \mathrm{s}\right)\end{array} & \text { Date } & \begin{array}{r}\text { Discharge } \\ \left(\mathrm{ft}^{3} / \mathrm{s}\right)\end{array} \\ 2-25-58 & 13.6 & 4-7-58 & 18.7 & 7-11-60 & 3.05\end{array}$


Captain Creek near Wellston, Oklahoma, Miscellaneous site.

Lat. $35^{\circ} 42^{\prime} 00^{\prime \prime}$, long. $97^{\circ} 04^{\prime} 00^{\prime \prime}, \mathrm{NE} 1 / 4 \mathrm{sec} .15, \mathrm{~T} .14 \mathrm{~N}$., R.2 E., at bridge on U.S. Highway $66,1 / 2$ mile west of wellston, Oklahoma.'

\begin{tabular}{|c|c|c|c|c|c|c|c|}
\hline Date & $\begin{array}{c}\text { Discharge } \\
\left(\mathrm{ft}^{3} / \mathrm{s}\right)\end{array}$ & Date & $\begin{array}{c}\text { Discharge } \\
\left(\mathrm{ft}^{3} / \mathrm{s}\right)\end{array}$ & Date & $\begin{array}{c}\text { Discharge } \\
\left(\mathrm{ft}^{3} / \mathrm{s}\right)\end{array}$ & Date & $\begin{array}{c}\text { Discharge } \\
\left(\mathrm{ft}^{3} / \mathrm{s}\right)\end{array}$ \\
\hline $\begin{array}{r}11-17-53 \\
12-19-53 \\
2-1-54 \\
3-9-54 \\
4-19-54 \\
6-1-54 \\
7-12-54 \\
8-20-54 \\
10-5-54\end{array}$ & $\begin{array}{l}2.82 \\
2.24 \\
2.85 \\
2.86 \\
1.27 \\
2.39 \\
0 \\
0 \\
0\end{array}$ & $\begin{array}{r}12-15-54 \\
2-15-55 \\
4-11-55 \\
5-31-55 \\
7-11-55 \\
8-17-55 \\
10-11-55 \\
10-26-55 \\
11-7-55\end{array}$ & $\begin{array}{l}0 \\
0 \\
1.88 \\
1.41 \\
0 \\
0 \\
0 \\
0 \\
0\end{array}$ & $\begin{array}{r}11-21-55 \\
1-6-56 \\
1-24-56 \\
2-29-56 \\
4-10-56 \\
5-14-56 \\
6-11-56 \\
7-3-56 \\
7-13-56\end{array}$ & $\begin{array}{l}0 \\
0 \\
1.16 \\
.33 \\
1.42 \\
0 \\
0 \\
0 \\
0\end{array}$ & $\begin{array}{r}8-13-56 \\
8-27-56 \\
9-29-56 \\
11-14-56 \\
2-6-57 \\
3-25-57 \\
6-25-57 \\
9-5-57\end{array}$ & $\begin{array}{l}0 \\
n \\
0 \\
0 \\
0 \\
24.15 \\
0\end{array}$ \\
\hline
\end{tabular}

Cavalry Creek near Cordell, Oklahoma, Miscellaneous site.

Lat. $35^{\circ} 17^{\prime} 18^{\prime \prime}$, long. $98^{\circ} 51^{\prime} 25^{\prime \prime}$, SW $1 / 4$ sec.1, T.9 N., R. 16 W., at bridge on State Highway 54,8 miles east of Cordeli, Okla.
Date Discharge
$\left(\mathrm{ft}^{3} / \mathrm{s}\right)$
$7-9-52 \quad .98$

Cedar Creek near Carnegie, Oklahoma, Miscellaneous site.

Lat. $35^{\circ} 09^{\prime} 00^{\prime \prime}$, long $98^{\circ} 35^{\prime} 00^{\prime \prime}$, SW $1 / 4$ sec. 29, T. $8 \mathrm{~N} .$, R. $13 \mathrm{~W}$, at bridge on State Highway $58,2 \mathrm{miles}$ north of Carnegie, Okla.

\begin{tabular}{|c|c|c|c|c|c|c|c|}
\hline Date & $\begin{array}{c}\text { Discharge } \\
\left(\mathrm{ft}^{3} / \mathrm{s}\right)\end{array}$ & Date & $\begin{array}{c}\text { Discharge } \\
\left(\mathrm{ft}^{3} / \mathrm{s}\right)\end{array}$ & Date & $\begin{array}{c}\text { Discharge } \\
\left(\mathrm{ft}^{3} / \mathrm{s}\right)\end{array}$ & Date & $\begin{array}{c}\text { Discharge } \\
\left(\mathrm{ft}^{3} / \mathrm{s}\right)\end{array}$ \\
\hline $\begin{array}{l}7-9-52 \\
9-15-53\end{array}$ & $\begin{array}{l}2.10 \\
1.12\end{array}$ & $3-16-55$ & 2.56 & $8-30-56$ & .80 & $2-19-59$ & 2.67 \\
\hline
\end{tabular}

Chisholm Creek near Seward, Oklahoma, Miscellaneous site.

Lat $35^{\circ} 46^{\prime} 00^{\prime \prime}$, long. $97^{\circ} 31^{\prime} 30^{\prime \prime}$, SE $1 / 4$ sec.9, T.15 N., R.3 S., 1-1/2 miles southwest of Seward, Okla.

\begin{tabular}{|c|c|c|c|c|c|c|c|}
\hline Date & $\begin{array}{c}\text { Discharge } \\
\left(\mathrm{ft}^{3} / \mathrm{s}\right)\end{array}$ & Date & $\begin{array}{c}\text { Dishcarge } \\
\left(\mathrm{ft}^{3} / \mathrm{s}\right)\end{array}$ & Date & $\begin{array}{c}\text { Discharge } \\
\left(\mathrm{ft}^{3} / \mathrm{s}\right)\end{array}$ & Date & $\begin{array}{c}\text { Discharge } \\
\left(\mathrm{ft}^{3} / \mathrm{s}\right)\end{array}$ \\
\hline $\begin{array}{r}5-16-41 \\
7-9-41 \\
9-23-41 \\
11-16-42 \\
12-14-42 \\
2-5-43 \\
3-9-43 \\
4-15-43 \\
6-30-43 \\
9-25-43\end{array}$ & $\begin{array}{l}5.10 \\
1.77 \\
2.79 \\
2.08 \\
2.41 \\
4.57 \\
1.07 \\
3.08 \\
1.06\end{array}$ & $\begin{array}{r}11-18-43 \\
3-9-44 \\
4-4-44 \\
5-18-44 \\
6-27-44 \\
7-19-44 \\
8-24-44 \\
10-16-44 \\
11-17-44\end{array}$ & $\begin{array}{r}.86 \\
1.58 \\
2.55 \\
3.40 \\
1.62 \\
2.47 \\
0 \\
1.92 \\
.31\end{array}$ & $\begin{array}{r}1-26-45 \\
2-24-45 \\
10-23-45 \\
11-20-45 \\
2-8-46 \\
3-20-46 \\
6-5-46 \\
7-24-46 \\
9-16-46\end{array}$ & $\begin{array}{l}1.26 \\
1.40 \\
1.90 \\
1.92 \\
.99 \\
6.52 \\
2.47 \\
.15\end{array}$ & $\begin{array}{r}11-29-46 \\
2-14-47 \\
3-27-47 \\
6-16-47 \\
8-27-47 \\
10-23-59 \\
10-29-59 \\
12-14-59 \\
5-16-60\end{array}$ & $\begin{array}{l}.95 \\
1.37 \\
1.31 \\
3.31 \\
0 \\
6.26 \\
5.12 \\
4.65 \\
6.20\end{array}$ \\
\hline
\end{tabular}

Chisholm Creek near Edmond, Oklahoma, Miscellaneous site.

Lat $34^{\circ} 41^{\prime} 00^{\prime \prime}$, long $97^{\circ} 30^{\prime} 00^{\prime \prime}, \mathrm{SW}^{\prime} 1 / 4 \mathrm{sec} .28, \mathrm{~T} .14 \mathrm{~N} ., \mathrm{R} .3 \mathrm{~W} ., 2$ miles west of Edmond, Oklahoma.

\begin{tabular}{|c|c|c|c|c|c|c|c|}
\hline Date & $\begin{array}{c}\text { Discharge } \\
\left(\mathrm{ft}^{3} / \mathrm{s}\right)\end{array}$ & Date & $\begin{array}{c}\text { Discharge } \\
\left(\mathrm{ft}^{3} / \mathrm{s}\right)\end{array}$ & Date & $\begin{array}{c}\text { Discharge } \\
\left(\mathrm{ft}^{3} / \mathrm{s}\right)\end{array}$ & Date & $\begin{array}{c}\text { Discharge } \\
\left(\mathrm{ft}^{3} / \mathrm{s}\right)\end{array}$ \\
\hline $\begin{array}{l}5-16-41 \\
7-9-41 \\
9-23-41\end{array}$ & $\begin{array}{l}.31 \\
.03 \\
.38\end{array}$ & $\begin{array}{r}11-16-42 \\
12-14-42 \\
2-5-43\end{array}$ & $\begin{array}{l}.12 \\
.50 \\
.52\end{array}$ & $\begin{array}{l}3-9-43 \\
4-15-43 \\
6-30-43\end{array}$ & $\begin{array}{l}.41 \\
.25 \\
.15\end{array}$ & $\begin{array}{r}9-25-43 \\
10-23-59\end{array}$ & .15 \\
\hline
\end{tabular}


Chuckwa Creek near Durant, Oklahoma, Miscellaneous site.

Lat. $34^{\circ} 00^{\prime} 00^{\prime \prime}$, long. $96^{\circ} 25^{\prime} 43^{\prime \prime}$, SE $1 / 4 \mathrm{sec} .24$, T.6 S., R. 8 E., at bridge on county road, $1-3 / 4 \mathrm{miles}$ northwest of Durant, okla.

Date Discharge
$\left(\mathrm{ft}^{3} / \mathrm{s}\right)$

$10-8-52$

Clear Boggy Creek near Frisco, Oklahoma, Miscellaneous site.

Lat $34^{\circ} 38^{\prime} 45^{\prime \prime}$, long $96^{\circ} 35^{\prime}, \mathrm{NW} 1 / 4 \mathrm{sec} .16, \mathrm{~T} .2 \mathrm{~N} ., \mathrm{R} .7 \mathrm{E}$. , at Frisco, Okla.

$\begin{array}{lrcccccc}\text { Date } & \begin{array}{c}\text { Discharge } \\ \left(\mathrm{ft}^{3} / \mathrm{s}\right)\end{array} & \text { Date } & \begin{array}{c}\text { Discharge } \\ \left(\mathrm{ft}^{3} / \mathrm{s}\right)\end{array} & \text { Date } & \begin{array}{c}\text { Discharge } \\ \left(\mathrm{ft}^{3} / \mathrm{s}\right)\end{array} & \begin{array}{c}\text { Date } \\ \text { Discharge } \\ \left(\mathrm{ft}^{3} / \mathrm{s}^{2}\right)\end{array} \\ 9-8-54 & 1.45 & 6-19-56 & .01 & 2-24-58 & 27.7 & 1-28-59 & 5.34 \\ 9-21-54 & .98 & 12-12-57 & 20.6 & 4-7-58 & 42.1 & \end{array}$

Clear Boggy Creek near Tupelo, Oklahoma, 07334400 , partial record site.

Lat. $34^{\circ} 32^{\prime} 45^{\prime \prime}$, long. $96^{\circ} 24^{\prime} 30^{\prime \prime}$, in NE $1 / 4 \mathrm{sec} .24$, T.1 N., R. 8 E., Coal County, at bridge on State Highway 48, 700 feet downstream from Goose Creek and 4 miles south of Tupelo.

\begin{tabular}{|c|c|c|c|c|c|c|c|}
\hline Date & $\begin{array}{c}\text { Discharge } \\
\left(\mathrm{ft}^{3} / \mathrm{s}\right)\end{array}$ & Date & $\begin{array}{c}\text { Nischarge } \\
\left(\mathrm{ft}^{3} / \mathrm{s}\right)\end{array}$ & Date & $\begin{array}{c}\text { Discharge } \\
\left(\mathrm{ft}^{3} / \mathrm{s}\right)\end{array}$ & Date & $\begin{array}{c}\text { Discharge } \\
\left(\mathrm{ft}^{3} / \mathrm{s}\right)\end{array}$ \\
\hline $\begin{array}{r}12-12-57 \\
2-24-58 \\
3-7-58 \\
11-4-58 \\
9-14-59 \\
10-26-59 \\
12-7-59 \\
1-25-60 \\
3-21-60 \\
9-13-60 \\
11-29-60 \\
1-10-61 \\
2-27-61 \\
5-15-61\end{array}$ & $\begin{array}{r}71.7 \\
80.1 \\
103 . \\
15.1 \\
10.5 \\
33.0 \\
37.0 \\
135.0 \\
95.0 \\
18.5 \\
77.8 \\
83.0 \\
85.0 \\
38.4\end{array}$ & $\begin{array}{r}9-11-61 \\
10-31-61 \\
5-16-62 \\
7-11-62 \\
8-15-62 \\
9-19-62 \\
12-19-62 \\
2-8-63 \\
4-22-63 \\
8-19-63 \\
10-11-63 \\
12-2-63 \\
2-17-64 \\
9-15-64\end{array}$ & $\begin{array}{c}59.0 \\
70.2 \\
58.4 \\
21.2 \\
14.7 \\
64.9 \\
74.6 \\
59.6 \\
42.5 \\
7.57 \\
5.77 \\
15.7 \\
5.75 \\
6.10\end{array}$ & $\begin{array}{r}3-9-65 \\
5-24-65 \\
11-22-65 \\
3-17-66 \\
6-28-66 \\
11-22-66 \\
2-13-67 \\
6-16-67 \\
8-29-67 \\
12-6-67 \\
2-19-68 \\
6-20-68 \\
9-11-68 \\
12-10-68\end{array}$ & $\begin{array}{r}43.6 \\
48.8 \\
11.0 \\
29.0 \\
4.8 \\
6.8 \\
7.6 \\
36.0 \\
7.0 \\
36.0 \\
100.0 \\
82.0 \\
29.0 \\
80.0\end{array}$ & $\begin{array}{r}6-10-60 \\
8-11-69 \\
12-11-69 \\
2-20-70 \\
5-20-70 \\
7-16-70 \\
12-1-70 \\
3-4-71 \\
6-28-71 \\
7-29-71 \\
1-26-72 \\
2-8-72 \\
4-18-72 \\
8-2-72\end{array}$ & $\begin{array}{l}87.0 \\
20.0 \\
50.0 \\
42.0 \\
60.0 \\
22.0 \\
64.0 \\
54.0 \\
22.0 \\
10.0 \\
50.0 \\
49.0 \\
27.0 \\
5.3\end{array}$ \\
\hline
\end{tabular}

Clear Creek near Beaver, Oklahoma, Miscellaneous site.

Lat. $36^{\circ} 38^{\prime 4} 9^{\prime \prime}$, long. $100^{\circ} 322^{\prime} 00^{\prime \prime}$, east line sec.13, T.2 N., R. 23 E., at bridge on U.S. Highway 270 , 12 miles south of Beaver, Oklahoma.

$\begin{array}{lrrr}\text { Date } & \begin{array}{c}\text { Discharge } \\ \left(\mathrm{ft}^{3} / \mathrm{s}\right)\end{array} & \text { Date } & \begin{array}{r}\text { Nischarge } \\ \left(\mathrm{ft}^{3} / \mathrm{s}\right)\end{array} \\ 7-7-49 & .81 & 4-11-50 & 1.19\end{array}$

Clear Creek near Elmwood, Oklahoma, Miscellaneous site.

Lat. $34^{\circ} 39^{\prime} 00^{\prime \prime}$, long. $100^{\circ} 30^{\prime} 00^{\prime \prime}$, east line sec.13, T.2 N., R.23 E., at bridge on U.S. Highway 270 , 3 miles southwest of Elmwood, Oklahoma.

\begin{tabular}{|c|c|c|c|c|c|c|c|}
\hline Date & $\begin{array}{c}\text { Discharge } \\
\left(\mathrm{ft}^{3} / \mathrm{s}\right)\end{array}$ & Date & $\begin{array}{c}\text { Discharge } \\
\left(\mathrm{ft}^{3} / \mathrm{s}\right)\end{array}$ & Date & $\begin{array}{c}\text { Discharge } \\
\left(\mathrm{ft}^{3} / \mathrm{s}\right)\end{array}$ & Date & $\begin{array}{c}\text { Discharge } \\
\left(\mathrm{ft}^{3} / \mathrm{s}\right)\end{array}$ \\
\hline $\begin{array}{r}10-8-51 \\
1-7-52\end{array}$ & $\begin{array}{l}1.11 \\
1.61\end{array}$ & $2-28-52$ & 1.63 & $4-17-52$ & 2.04 & $\begin{array}{l}6-19-52 \\
8-11-52\end{array}$ & .72 \\
\hline
\end{tabular}


Clear Creek near May, Oklahoma, 07234300 , partial record site.

Lat. $36^{\circ} 39^{\prime} 22^{\prime \prime}$, Jong. $99^{\circ} 51^{\prime} 00^{\prime \prime}$, in SW 1/4 sec. 24, T.25 N., R. 25 W., Harper County, at bridge on U.S. Highway $270,8.5$ miles west of May.

\begin{tabular}{|c|c|c|c|c|c|c|c|}
\hline Date & $\begin{array}{c}\text { Discharge } \\
\left(\mathrm{ft}^{3} / \mathrm{s}\right)\end{array}$ & Date & $\begin{array}{c}\text { Nischarge } \\
\left(\mathrm{ft}^{3} / \mathrm{s}\right)\end{array}$ & Date & $\begin{array}{c}\text { Discharge } \\
\left(\mathrm{ft}^{3} / \mathrm{s}\right)\end{array}$ & Date & $\begin{array}{c}\text { Discharge } \\
\left(\mathrm{ft}^{3} / \mathrm{s}\right)\end{array}$ \\
\hline $\begin{array}{r}12-31-53 \\
2-18-54 \\
9-8-54 \\
11-17-54 \\
1-24-55 \\
3-29-55 \\
7-28-55 \\
10-19-55 \\
3-21-56 \\
8-15-56 \\
9-25-56 \\
12-20-56 \\
9-26-57 \\
10-30-57 \\
12-2-57 \\
3-26-58 \\
7-25-58 \\
10-31-58 \\
2-25-59 \\
4-20-59 \\
7-14-59\end{array}$ & $\begin{array}{l}5.59 \\
9.23 \\
2.12 \\
6.54 \\
9.60 \\
8.70 \\
6.84 \\
7.02 \\
0.34 \\
3.11 \\
3.10 \\
7.75 \\
6.90 \\
11.7 \\
14.2 \\
10.5 \\
8.41 \\
8.28 \\
8.89 \\
7.01 \\
4.32\end{array}$ & $\begin{array}{r}8-2-59 \\
8-30-50 \\
10-29-59 \\
12-9-59 \\
1-6-60 \\
2-19-60 \\
3-25-60 \\
4-22-60 \\
5-27-60 \\
6-27-60 \\
8-22-60 \\
10-24-60 \\
1-6-61 \\
3-2-61 \\
4-20-61 \\
6-16-61 \\
7-21-61 \\
10-6-61 \\
1-16-52 \\
5-21-62 \\
8-13-62\end{array}$ & $\begin{array}{c}4.30 \\
8.05 \\
7.56 \\
6.77 \\
22.4 \\
14.3 \\
10.9 \\
8.00 \\
4.35 \\
1.88 \\
5.68 \\
6.53 \\
5.83 \\
9.16 \\
8.69 \\
8.16 \\
11.0 \\
8.45 \\
5.47 \\
4.18 \\
6.72\end{array}$ & $\begin{array}{r}3-15-63 \\
7-25-63 \\
10-17-63 \\
12-5-63 \\
2-13-64 \\
5-28-64 \\
9-1-64 \\
12-29-64 \\
3-2-65 \\
4-27-65 \\
7-20-65 \\
10-7-65 \\
3-3-66 \\
3-8-66 \\
4-26-66 \\
7-6-66 \\
10-6-66 \\
1-17-67 \\
5-11-67 \\
7-28-67 \\
11-1-67\end{array}$ & $\begin{array}{l}8.88 \\
2.74 \\
7.73 \\
6.90 \\
9.63 \\
8.39 \\
3.88 \\
8.10 \\
21.5 \\
8.20 \\
13.5 \\
9.2 \\
9.7 \\
6.5 \\
8.1 \\
3.4 \\
4.6 \\
9.0 \\
4.7 \\
3.8 \\
5.6\end{array}$ & $\begin{array}{r}2-7-68 \\
4-10-68 \\
8-9-68 \\
11-20-68 \\
3-19-69 \\
6-26-69 \\
8-6-69 \\
12-17-69 \\
2-26-70 \\
4-16-70 \\
7-24-70 \\
11-6-70 \\
3-11-71 \\
5-28-71 \\
9-9-71 \\
12-17-71 \\
3-16-72 \\
6-8-72 \\
12-21-72 \\
3-1-73 \\
5-22-73\end{array}$ & $\begin{array}{r}8.4 \\
6.8 \\
5.4 \\
7.5 \\
12.0 \\
7.0 \\
3.2 \\
6.4 \\
6.5 \\
7.2 \\
3.9 \\
7.0 \\
10.0 \\
7.3 \\
1.2 \\
7.9 \\
7.1 \\
3.6 \\
11.0 \\
9.6 \\
7.9\end{array}$ \\
\hline
\end{tabular}

Cobb Creek near Alfalfa, Oklahoma, Miscellaneous site.

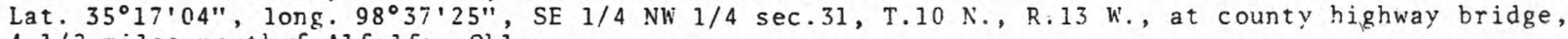
4-1/2 miles north of Alfalfa, Okla.
Date
Discharge
$\left(\mathrm{ft}^{3} / \mathrm{s}\right)$
Date
Discharge
$9-8-53$
2.01
$2-14-55$
6.25

Cobb Creek near Swan Lake, Oklahoma, Miscellaneous site.

Lat. $35^{\circ} 13^{\prime} 50^{\prime \prime}$, Iong. $98^{\circ} 32^{\prime} 7^{\prime \prime}$, SE $1 / 4$ SE $1 / 4 \mathrm{sec} .22$, T.9 N., R.13 K., at county highray bridge, 2 miles west of Swan Lake, Okla.

$\begin{array}{lrcc}\text { Date } & \begin{array}{c}\text { Discharge } \\ \left(\mathrm{ft}^{3} / \mathrm{s}\right)\end{array} & \text { Date } & \begin{array}{c}\text { Discharge } \\ \left(\mathrm{ft}^{3} / \mathrm{s}\right)\end{array} \\ 9-8-53 & 2.94 & 2-15-55 & 12.8\end{array}$

Cochran Creek near Marietta, Oklahoma, Miscellaneous site.

Lat. $33^{\circ} 54^{\prime} 41^{\prime \prime}$, long. $97^{\circ} 01^{\prime} 08^{\prime \prime}$, near center of south ine sec.29, T.7 S., R.3 E., 6-1/2 miles southeast of Marietta, oklahoma.

$\begin{array}{lr}\text { Date } & \begin{array}{r}\text { Discharge } \\ \left(\mathrm{ft}^{3} / \mathrm{s}\right)\end{array} \\ 3-23-49 & .16\end{array}$

Coffee Creek near Arcadia, Oklahoma, Miscellaneous site.

Lat. $35^{\circ} 39^{\prime} 41^{\prime \prime}, 1$ ang. $97^{\circ} 20^{\prime} 51^{\prime \prime}$, east line sec.30, T.14 N., R. I K., 2 miles southwest of Arcadia, oklahoma.

$\begin{array}{cr}\text { Date } & \begin{array}{r}\text { Discharge } \\ \left(\mathrm{ft}^{3} / \mathrm{s}\right)\end{array} \\ 8-15-50 & 4.89\end{array}$


Cold Springs Creek near Castaneda, Oklahoma, 07155200 , partial record site.

Lat. $36^{\circ} 52^{\prime} 30^{\prime \prime}$, long. $102^{\circ} 38^{\prime} 15^{\prime \prime}$, in SW $1 / 4 \mathrm{NW} 1 / 4 \mathrm{sec} .28,1.5 \mathrm{~N}$, , R. $4 \mathrm{E}$., Cimarron County, at county road bridge, 6 miles west of Castaneda and 9.7 miles northwest of Boise City, Okla.

\begin{tabular}{|c|c|c|c|c|c|c|c|}
\hline Date & $\begin{array}{c}\text { Discharge } \\
\left(\mathrm{ft}^{3} / \mathrm{s}\right)\end{array}$ & Date & $\begin{array}{c}\text { Discharge } \\
\left(\mathrm{ft}^{3} / \mathrm{s}\right)\end{array}$ & Date & $\begin{array}{c}\text { Discharge } \\
\left(\mathrm{ft}^{3} / \mathrm{s}\right)\end{array}$ & Date & $\begin{array}{c}\text { Discharge } \\
\left(\mathrm{ft}^{3} / \mathrm{s}\right)\end{array}$ \\
\hline $\begin{array}{r}11-23-66 \\
1-16-67 \\
4-24-67 \\
7-24-67 \\
2-26-68\end{array}$ & $\begin{array}{l}0.07 \\
0 \\
0 \\
.05\end{array}$ & $\begin{array}{r}5-20-68 \\
7-23-68 \\
12-11-68 \\
3-11-68 \\
5-27-68\end{array}$ & $\begin{array}{l}0 \\
0 \\
0 \\
0 \\
0\end{array}$ & $\begin{array}{r}8-11-68 \\
12-3-69 \\
3-9-70 \\
5-19-70 \\
7-30-70\end{array}$ & $\begin{array}{l}0 \\
0 \\
0 \\
0\end{array}$ & $\begin{array}{r}11-10-70 \\
3-1-71 \\
6-22-71 \\
9-14-71\end{array}$ & $\begin{array}{l}0 \\
0 \\
0 \\
0\end{array}$ \\
\hline
\end{tabular}

Commission Creek near Grand, Oklahoma, 07228150 , partial record site.

Lat. $36^{\circ} 02^{\prime} 00^{\prime \prime}$, long. $99^{\circ} 55^{\prime} 00^{\prime \prime}$, west line of SW $1 / 4 \mathrm{SE} 1 / 4 \mathrm{sec} .18, \mathrm{~T} .18 \mathrm{~N}$. , R.25 W., at bridge on State Highway 46 , half a mile upstream from Hog Creek and 8 miles northwest of Grand, Okla.

\begin{tabular}{|c|c|c|c|c|c|c|c|}
\hline Date & $\begin{array}{c}\text { Discharge } \\
\left(\mathrm{ft}^{3} / \mathrm{s}\right)\end{array}$ & Date & $\begin{array}{c}\text { Discharge } \\
\left(\mathrm{ft}^{3} / \mathrm{s}\right)\end{array}$ & Date & $\begin{array}{c}\text { Discharge } \\
\left(\mathrm{ft}^{3} / \mathrm{s}\right)\end{array}$ & Date & $\begin{array}{c}\text { Discharge } \\
\left(\mathrm{ft}^{3} / \mathrm{s}\right)\end{array}$ \\
\hline $\begin{array}{r}10-26-64 \\
3-26-65\end{array}$ & $\begin{array}{l}2.64 \\
5.47\end{array}$ & $11-9-65$ & 5.50 & $2-17-66$ & 6.0 & $5-12-66$ & 4.4 \\
\hline
\end{tabular}

Cottonwood Creek near Guthrie, Oklahoma, 07159800 , partial record site.

Lat. $35^{\circ} 52^{\prime} 45^{\prime \prime}$, long. $97^{\circ} 26^{\circ} 30^{\prime \prime}$, in SE $1 / 4$ sec.18, T.16 N., R.2 W., Logan County, at county road hridge, 2 miles southwest of Guthrie, Okla.

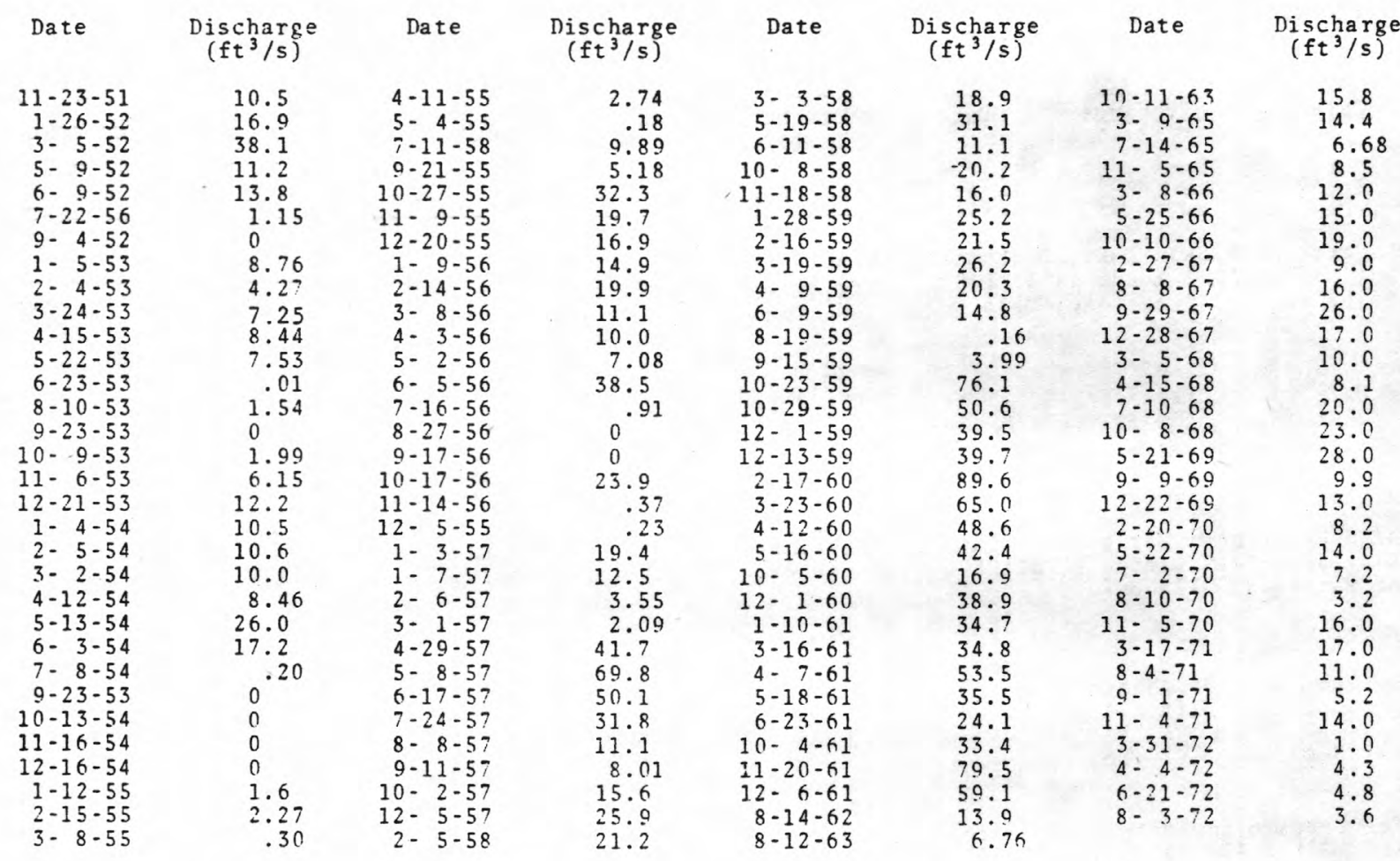

Cottonwood Creek near Seward, Oklahoma, 07159750, partial record site.

Lat $35^{\circ} 47^{\prime} 00^{\prime \prime}$, long. $97^{\circ} 32^{\prime} 00^{\prime \prime}, \mathrm{SW} i / 4 \mathrm{sec} .9, \mathrm{~T} .15 \mathrm{~N} ., \mathrm{R} .3 \mathrm{~W} ., 2$ miles west of Seward, Okla.

\begin{tabular}{|c|c|c|c|c|c|c|c|}
\hline Date & $\begin{array}{c}\text { Discharge } \\
\left(\mathrm{ft}^{3} / \mathrm{s}\right)\end{array}$ & Date & $\begin{array}{c}\text { Discharge } \\
\left(\mathrm{ft}^{3} / \mathrm{s}\right)\end{array}$ & Date & $\begin{array}{c}\text { Discharge } \\
\left(\mathrm{ft}^{3} / \mathrm{s}\right)\end{array}$ & Date & $\begin{array}{c}\text { Discharge } \\
\left(\mathrm{ft}^{3} / \mathrm{s}\right)\end{array}$ \\
\hline $\begin{array}{l}5-16-41 \\
7-9-41 \\
9-23-41\end{array}$ & $\begin{array}{l}42.4 \\
12.7 \\
14.0\end{array}$ & $\begin{array}{r}11-16-42 \\
12-14-42 \\
2-5-43\end{array}$ & $\begin{array}{l}9.47 \\
12.6 \\
20.4\end{array}$ & $\begin{array}{l}4-15-43 \\
6-30-43 \\
9-25-43\end{array}$ & $\begin{array}{c}15.0 \\
3.94 \\
0\end{array}$ & $\begin{array}{l}11-18-43 \\
12-13-59\end{array}$ & $\begin{array}{l}2.30 \\
34.6\end{array}$ \\
\hline
\end{tabular}


Cottonwood Creek near Navina, Oklahoma, Miscellaneous site.

Lat. $35^{\circ} 46^{\prime} 30^{\prime \prime}$, long. $97^{\circ} 35^{\prime} 00^{\prime \prime}$, NW'1/4 SW $1 / 4$ sec.13, T.15 N., R.4 W., 2 miles southwest of Navina, 0k1 a.

\begin{tabular}{|c|c|c|c|c|c|c|c|}
\hline Date & $\begin{array}{c}\text { Discharge } \\
\left(\mathrm{ft}^{3} / \mathrm{s}\right)\end{array}$ & Date & $\begin{array}{c}\text { Di scharge } \\
\left(\mathrm{ft}^{3} / \mathrm{s}\right)\end{array}$ & Date & $\begin{array}{c}\text { Discharge } \\
\left(\mathrm{ft}^{3} / \mathrm{s}\right)\end{array}$ & Date & $\begin{array}{c}\text { Discharge } \\
\left(\mathrm{ft}^{3} / \mathrm{s}\right)\end{array}$ \\
\hline $\begin{array}{r}3-9-44 \\
4-4-44 \\
6-27-44 \\
7-19-44 \\
8-24-44 \\
10-16-44 \\
11-15-44 \\
1-26-45 \\
2-23-45 \\
4-7-45\end{array}$ & $\begin{array}{l}3.68 \\
6.75 \\
1.90 \\
.78 \\
0 \\
5.33 \\
1.48 \\
3.22 \\
4.72 \\
4.24\end{array}$ & $\begin{array}{r}5-22-45 \\
6-20-45 \\
7-19-45 \\
8-24-45 \\
10-23-45 \\
11-20-45 \\
2-8-46 \\
3-20-46 \\
4-30-46\end{array}$ & $\begin{array}{l}7.77 \\
2.54 \\
15.8 \\
1.91 \\
4.75 \\
4.31 \\
2.23 \\
4.31 \\
3.64\end{array}$ & $\begin{array}{r}6-5-46 \\
7-24-46 \\
9-16-46 \\
11-29-46 \\
2-14-47 \\
3-27-47 \\
6-16-47 \\
8-27-47 \\
4-12-49\end{array}$ & $\begin{array}{l}1.70 \\
0 \\
0 \\
1.90 \\
1.84 \\
1.04 \\
3.68 \\
8.00 \\
4.05\end{array}$ & $\begin{array}{r}9-26-49 \\
3-9-50 \\
9-8-50 \\
8-20-51 \\
1-28-52 \\
7-28-52 \\
10-23-59 \\
10-29-59 \\
12-14-59 \\
5-15-60\end{array}$ & $\begin{array}{l}0.08 \\
3.07 \\
2.30 \\
3.22 \\
0 \\
15.8 \\
11.5 \\
7.60 \\
9.01\end{array}$ \\
\hline
\end{tabular}

Cow Creek at Waurika, Oklahoma, 07313600 , partial record site.

Lat $34^{\circ} 10^{\prime} 55^{\prime \prime}$, long. $98^{\circ} 00^{\prime} 05^{\prime \prime}$, in SE $1 / 4 \mathrm{NE} 1 / 4 \mathrm{sec} .26, \mathrm{~T} .4 \mathrm{~S}$. , R. 8 W., Jefferson County, at

Chicago, Rock Island and Pacific Railway Co. bridge, near north edge of Waurika, and at mile 1.7 . (Regular gaging station 1966-1970)

\begin{tabular}{|c|c|c|c|c|c|c|c|}
\hline Date & $\begin{array}{c}\text { Discharge } \\
\left(\mathrm{ft}^{3} / \mathrm{s}\right)\end{array}$ & Date & $\begin{array}{c}\text { Discharge } \\
\left(\mathrm{ft}^{3} / \mathrm{s}\right)\end{array}$ & Date & $\begin{array}{c}\text { Discharge } \\
\left(\mathrm{ft}^{3} / \mathrm{s}\right)\end{array}$ & Date & $\begin{array}{c}\text { Dishcarge } \\
\left(\mathrm{ft}^{3} / \mathrm{s}\right)\end{array}$ \\
\hline $\begin{array}{r}6-15-66 \\
7-16-66 \\
8-9-66 \\
11-1-66 \\
8-19-67 \\
9-26.67 \\
10-25-67\end{array}$ & $\begin{array}{l}1.1 \\
0 \\
.70 \\
3.5 \\
.87 \\
2.9 \\
1.6\end{array}$ & $\begin{array}{r}7-27-68 \\
8-27-68 \\
8-1-69 \\
8-21-69 \\
10-23-69 \\
8-11-69 \\
10-28-69\end{array}$ & $\begin{array}{l}1.4 \\
1.2 \\
1.8 \\
1.5 \\
2.4 \\
.08 \\
4.30\end{array}$ & $\begin{array}{r}7-20-69 \\
8-6-69 \\
8-16-69 \\
11-18-70 \\
1-20-71 \\
4-6-71 \\
7-21-71\end{array}$ & $\begin{array}{l}1.25 \\
.23 \\
.01 \\
7.5 \\
4.4 \\
4.2 \\
1.9\end{array}$ & $\begin{array}{r}10-19-71 \\
1-12-72 \\
4-4-72 \\
7-19-72 \\
10-11-72 \\
2-21-73 \\
5-15-73\end{array}$ & $\begin{array}{l}3.8 \\
5.4 \\
3.9 \\
3.6\end{array}$ \\
\hline
\end{tabular}

Crooked Creek near Gate, Oklahoma, Miscellaneous site.

Lat. $34^{\circ} 58^{\prime} 37^{\prime \prime}$, long. $94^{\circ} 26^{\prime} 54^{\prime \prime}$, SE $1 / 4$ sec.26, T.6 N., R. 27 E. at mouth, 7 miles northof Gate, Okla.

$\begin{array}{lcccccc}\text { Date } & \begin{array}{c}\text { Discharge } \\ \left(\mathrm{ft}^{3} / \mathrm{s}\right)\end{array} & \text { Date } & \begin{array}{c}\text { Discharge } \\ \left(\mathrm{ft}^{3} / \mathrm{s}\right)\end{array} & \text { Date } & \begin{array}{c}\text { Discharge } \\ \left(\mathrm{ft}^{3} / \mathrm{s}\right)\end{array} & \begin{array}{c}\text { Date } \\ 3-31-43\end{array} \\ 9.19 & 6-17-43 & 6.43 & 7-7-43 & 0 & 7-22-43\end{array}$

Cummings' Spring near Connerville, Oklahoma, Miscellaneous site.

Lat. $34^{\circ} 26^{\prime} 04^{\prime \prime}$, long. $96^{\circ} 39^{\prime} 25^{\prime \prime}$, NE $1 / 4 \mathrm{SH} 1 / 4 \mathrm{sec} .24, \mathrm{~T} .1 \mathrm{~S} ., \mathrm{R} .6 \mathrm{E} ., 150 \mathrm{ft}$ below spring, $1 / 2$ mile northeast of Connerville, Okla.

$\begin{array}{rr}\text { Date } & \begin{array}{r}\text { Discharge } \\ \left(\mathrm{ft}^{3} / \mathrm{s}\right)\end{array} \\ 6-19-56 & 2.83\end{array}$

Dead Indian Creek near Cheyenne, Oklahoma, Miscellaneous site.

Lat. $35^{\circ} 43^{\prime} 34^{\prime \prime}$, long. $98^{\circ} 38^{\prime}$ i $7^{\prime \prime}$, west line sec.6, T.14 N., R.13 W., at bridge on U.S. Highway 283 , 7 miles north of Cheyenne, oklahoma.

$\begin{array}{lr}\text { Date } & \begin{array}{r}\text { Discharge } \\ \left(\mathrm{ft}^{3} / \mathrm{s}\right)\end{array} \\ 9-14-47 & .59\end{array}$


Dead Indian Creek near Roll, Oklahoma, Miscellaneous site.

Lat. $35^{\circ} 46^{\prime} 23^{\prime \prime}$, long. $99^{\circ} 45^{\prime} 25^{\prime \prime}$, on north line of sec.22, T.15 N., R. $24 \mathrm{~h}^{\prime}$, at county highway bridge, 2 miles southwest of Roll, Okla.

$\begin{array}{crrr}\text { Date } & \begin{array}{c}\text { Discharge } \\ \left(\mathrm{ft}^{3} / \mathrm{s}\right)\end{array} & \text { Date } & \begin{array}{r}\text { Discharge } \\ \left(\mathrm{ft}^{3} / \mathrm{s}\right)\end{array} \\ 9-13-56 & .11 & 3-20-56 & .94\end{array}$

Deep Creek near Sayre, Oklahoma, Miscellaneous site.

Lat. " $35^{\circ} 17^{\prime} 18^{\prime \prime}$, long. $99^{\circ} 36^{\prime} 34^{\prime \prime}$, on north line, sec.2, T.9 N., R.23 W., at bridge on State Highway $152,1-1 / 2$ miles east of Sayre, Okla.
Date Discharge
$\left(\mathrm{ft}^{3} / \mathrm{s}\right)$
$2-14-57$
.06

Deep Creek near Seiling, Oklahoma, Miscellaneous site.

Lat. $36^{\circ} 11^{\prime} 32^{\prime \prime}$, long. $99^{\circ} 00^{\prime} 00^{\prime \prime}, \mathrm{SE} 1 / 4 \mathrm{sec} .26$, T. $20 \mathrm{~N}$., R.17 W., at bridge on U.S. Highway 270 , 3 miles northwest of Seiling, Okla.

$$
\text { Date Discharge }
$$

$9-28-48$

Deep Creek near Washita, Oklahoma, Miscellaneous site

Lat. $35^{\circ} 06^{\prime} 00^{\prime \prime}$, long. $98^{\circ} 22^{\prime} 51^{\prime \prime}$, NW $1 / 4 \mathrm{sec} .9$, T.7 N., R.11 W., at county highway bridge, $1-1 / 2 \mathrm{miles}$ west of Washita, Okia.

$\begin{array}{crcr}\text { Date } & \begin{array}{c}\text { Discharge } \\ \left(\mathrm{ft}^{3} / \mathrm{s}\right)\end{array} & \text { Date } & \begin{array}{r}\text { Discharge } \\ \left(\mathrm{ft}^{3} / \mathrm{s}\right)\end{array} \\ 7-9.52 & .57 & 10-1-52 & .20\end{array}$

Deep Fork near Arcadia, Oklahoma, Miscellaneous site.

Lat. $35^{\circ} 38^{\prime} 58^{\prime \prime}$, long. $97^{\circ} 21^{\prime} 41^{\prime \prime}, \mathrm{NW} 1 / 4 \mathrm{sec} .31$, T.14 N., R. $1 \mathrm{w}$. at county highway bridge $2 \mathrm{miles}$ southwest of Arcadia, Oklahoma.

$$
\begin{array}{cc}
\text { Date } & \begin{array}{c}
\text { Discharge } \\
\left(\mathrm{ft}^{3} / \mathrm{s}\right)
\end{array} \\
9-25-30 & 12.5
\end{array}
$$

Deep Fork near Stroud, Oklahoma, Miscellaneous site.

Lat. $35^{\circ} 41^{\prime} 32^{\prime \prime}$, long. $96^{\circ} 40^{\prime} 00^{\prime \prime}, \mathrm{NW} 1 / 4 \mathrm{SW}^{\circ} 1 / 4 \mathrm{sec} .15, \mathrm{~T} .14 . \mathrm{N} ., \mathrm{R} .6 \mathrm{E}$. at bridge on State Highway $99,4-1 / 2$ miles south of Stroud, Okla.

$$
\text { Date Discharge }
$$

$11-18-53 \quad 27.0$ 
Deep Fork near Welty, Oklahoma, Miscellaneous site.

Lat. $35^{\circ} 40^{\circ}$, long. $96^{\circ} 20^{\circ}$, NK $1 / 4 \mathrm{NE} 1 / 4$ sec. 24 , T. 14 N., R. 8 E., at county highway bridge, 4 miles north of Welty, Okla.

\begin{tabular}{|c|c|c|c|c|c|c|c|}
\hline Date & $\begin{array}{c}\text { Discharge } \\
\left(\mathrm{ft}^{3} / \mathrm{s}\right)\end{array}$ & Date & $\begin{array}{c}\text { Discharge } \\
\left(\mathrm{ft}^{3} / \mathrm{s}\right)\end{array}$ & Date & $\begin{array}{c}\text { Discharge } \\
\left(\mathrm{ft}^{3} / \mathrm{s}\right)\end{array}$ & Date & $\begin{array}{c}\text { Discharge } \\
\left(\mathrm{ft}^{3} / \mathrm{s}\right)\end{array}$ \\
\hline $\begin{array}{r}12-28-53 \\
12-29-53 \\
2-1-54 \\
3-9-54 \\
4-19-54 \\
6-1-54 \\
7-12-54\end{array}$ & $\begin{array}{r}39.0 \\
37.1 \\
40.3 \\
34.9 \\
36.8 \\
146.0 \\
2.45\end{array}$ & $\begin{array}{r}8-20-54 \\
10-5-54 \\
11-18-54 \\
1-17-55 \\
3-2-55 \\
4-11-55 \\
5-31-55\end{array}$ & $\begin{array}{l}2.40 \\
0 \\
0 \\
12.9 \\
17.2 \\
20.6 \\
385.0\end{array}$ & $\begin{array}{r}7-11-55 \\
8-17-55 \\
11-21-55 \\
1-16-56 \\
2-29-56 \\
4-10-56 \\
7-13-56\end{array}$ & $\begin{array}{l}18.1 \\
11.9 \\
15.2 \\
14.0 \\
21: 3 \\
45.4 \\
54.3\end{array}$ & $\begin{array}{r}8-27-56 \\
11-14-56 \\
1-3-57 \\
2-6-57 \\
3-25-57 \\
9-5-57\end{array}$ & $\begin{array}{c}0 \\
0 \\
18.0 \\
19.9 \\
17.8 \\
9.60\end{array}$ \\
\hline
\end{tabular}

Deep Red Run near Randlett, Oklahoma, Miscellaneous site.

Lat. $34^{\circ} 13^{\prime} 23^{\prime \prime}$, long. $98^{\circ} 26^{\prime} 34^{\prime \prime}$, SW $1 / 4$ sec.10, T. 4 S., R. 12 W., at bridge on U.S. Highway 277,3 miles north of Randlett, Okla.
Date Discharge
$8-17-49 \quad 1.98$

Deer Creek near Edmond, Oklahoma, Miscellaneous site.

Lat. $35^{\circ} 41^{\prime} 30^{\prime \prime}$, long. $97^{\circ} 35^{\prime} 30^{\prime \prime}, \mathrm{NW}^{\prime} 1 / 4 \mathrm{SW}^{\prime} 1 / 4 \mathrm{sec} .13$, T.14 N., R.4 K., 6 miles northwest of Edmond, oklahoma.

\begin{tabular}{|c|c|c|c|c|c|c|c|}
\hline Date & $\begin{array}{l}\text { Discharge } \\
\left(\mathrm{ft}^{3} / \mathrm{s}\right)\end{array}$ & Date & $\begin{array}{c}\text { Discharge } \\
\left(\mathrm{ft}^{3} / \mathrm{s}\right)\end{array}$ & Date & $\begin{array}{c}\text { Discharge } \\
\left(\mathrm{ft}^{3} / \mathrm{s}\right)\end{array}$ & Date & $\begin{array}{c}\text { Discharge } \\
\left(\mathrm{ft}^{3} / \mathrm{s}\right)\end{array}$ \\
\hline $\begin{array}{r}3-9-44 \\
4-4-44 \\
5-18-44 \\
6-27-44 \\
7-19-44 \\
8-24-44 \\
10-16-44 \\
11-15-44 \\
1-26-45 \\
2-23-45\end{array}$ & $\begin{array}{l}4.66 \\
9.04 \\
2.41 \\
0 \\
0 \\
0 \\
0 \\
0 \\
2.40 \\
4.21\end{array}$ & $\begin{array}{r}4-7-45 \\
5-22-45 \\
6-20-45 \\
7-19-45 \\
8-24-45 \\
10-23-45 \\
11-20-45 \\
2-8-46 \\
3-20-46 \\
4-30-46\end{array}$ & $\begin{array}{c}7.46 \\
22.2 \\
27.3 \\
47.7 \\
6.7 \\
19.5 \\
9.88 \\
10.2 \\
18.0 \\
9.29\end{array}$ & $\begin{array}{r}6-5-46 \\
7-24-46 \\
9-16-46 \\
11-29-46 \\
2-14-47 \\
3-27-47 \\
6-16-47 \\
8-27-47 \\
4-12-49 \\
9-26-49\end{array}$ & $\begin{array}{l}5.79 \\
.18 \\
0 \\
1.75 \\
3.03 \\
4.17 \\
9.14 \\
1.09 \\
8.37 \\
2.23\end{array}$ & $\begin{array}{r}3-9-50 \\
9-8-50 \\
8-20-51 \\
1-26-53 \\
7-23-53 \\
10-23-59 \\
10-29-59 \\
12-14-59 \\
5-16-60\end{array}$ & $\begin{array}{l}3.89 \\
4.27 \\
1.79 \\
2.33 \\
0 \\
26.4 \\
17.7 \\
14.9 \\
20.0\end{array}$ \\
\hline
\end{tabular}

Deer Creek near Hydro, Oklahoma, Miscellaneous site.

Lat. $35^{\circ} 33^{\prime} 00^{\prime \prime}$, long. $98^{\circ} 35^{\circ} 00^{\prime \prime}, \mathrm{NK} 1 / 4 \mathrm{SH} 1 / 4 \mathrm{sec} .4$, T.12 N., R.13 H. , at county highway bridge, $1 / 4$ mile south of Hydro, Okla.

\begin{tabular}{|c|c|c|c|c|c|c|c|}
\hline Date & $\begin{array}{c}\text { Discharge } \\
\left(\mathrm{ft}^{3} / \mathrm{s}\right)\end{array}$ & Date & $\begin{array}{c}\text { Discharge } \\
\left(\mathrm{ft}^{3} / \mathrm{s}\right)\end{array}$ & Date & $\begin{array}{c}\text { Discharge } \\
\left(\mathrm{ft}^{3} / \mathrm{s}\right)\end{array}$ & Date & $\begin{array}{c}\text { Discharge } \\
\left(\mathrm{ft}^{3} / \mathrm{s}\right)\end{array}$ \\
\hline $\begin{array}{r}8-6-51 \\
10-8-51 \\
11-28-51 \\
1-14-52 \\
3-4-52 \\
4-23-52 \\
6-6-52 \\
7-22-52 \\
8-19-52 \\
10-1-53 \\
11-2-53 \\
12-1-53 \\
1-18-54 \\
2-15-54 \\
3-15-54 \\
5-4-54 \\
6-2-54 \\
6-29-54 \\
7-27-54\end{array}$ & $\begin{array}{c}7.53 \\
14.4 \\
21.4 \\
22.6 \\
14.4 \\
74.3 \\
22.6 \\
1.81 \\
2.62 \\
3.48 \\
10.3 \\
14.2 \\
14.7 \\
14.8 \\
14.0 \\
13.7 \\
24.9 \\
8.25 \\
3.09\end{array}$ & $\begin{array}{r}8-26-54 \\
9-15-54 \\
10-18-54 \\
11-15-54 \\
12-15-54 \\
1-17-55 \\
2-14-55 \\
3-16-55 \\
4-19-55 \\
6-23-54 \\
7-21-55 \\
8-22-55 \\
10-18-55 \\
11-22-55 \\
12-15-55 \\
1-5-56 \\
1-31-56 \\
3-1-56\end{array}$ & $\begin{array}{c}3.01 \\
2.57 \\
2.83 \\
8.03 \\
9.60 \\
12.6 \\
13.3 \\
13.0 \\
12.2 \\
8.23 \\
21.3 \\
5.97 \\
26.0 \\
18.8 \\
17.7 \\
17.1 \\
4.02 \\
16.3\end{array}$ & $\begin{array}{r}5-7-56 \\
8-7-56 \\
9-10-56 \\
10-31-56 \\
11-27-56 \\
3-12-57 \\
4-2-57 \\
4-30-57 \\
5-8-57 \\
5-15-57 \\
7-1-57 \\
7-20-57 \\
8-16-57 \\
9-5-57 \\
11-14-57 \\
12-19-57 \\
1-28-58 \\
3-11-58\end{array}$ & $\begin{array}{c}10.7 \\
1.04 \\
1.53 \\
14.5 \\
9.80 \\
14.3 \\
16.5 \\
46.8 \\
35.4 \\
48.6 \\
20.4 \\
9.69 \\
7.78 \\
6.60 \\
17.7 \\
17.3 \\
17.3 \\
20.2\end{array}$ & $\begin{array}{r}3-30-58 \\
4-29-58 \\
5-21-58 \\
7-15-58 \\
8-27-58 \\
10-21-58 \\
12-2-58 \\
1-13-59 \\
2-17-59 \\
4-27-59 \\
6-0-59 \\
7-30-59 \\
8-27-59 \\
0-22-59 \\
10-20-59 \\
12-1-59 \\
7-8-60 \\
7-25-60 \\
9-1-60\end{array}$ & $\begin{array}{c}20.1 \\
20.9 \\
16.0 \\
11.8 \\
6.23 \\
7.44 \\
15.2 \\
16.8 \\
16.4 \\
17.2 \\
34.9 \\
49.1 \\
13.8 \\
13.5 \\
43.6 \\
33.8 \\
27.5 \\
27.2 \\
19.0\end{array}$ \\
\hline
\end{tabular}


Deer Creek near Tonkawa, Oklahoma, 07150900 partial record site.

Lat. $36^{\circ} 42^{\prime} 33^{\prime \prime}$, long. $97^{\circ} 22^{\prime} 22^{\prime \prime}$, on south line SW $1 / 4$ SE $1 / 4 \mathrm{sec} .23$, T. 26 N., R. 2 W., Kay County, at county road bridge, 4.5 miles northwest of Tonkawa and about 3.5 miles upstream from mouth.

\begin{tabular}{|c|c|c|c|c|c|c|c|}
\hline Date & $\begin{array}{c}\text { Discharge } \\
\left(f t^{3} / \mathrm{s}\right)\end{array}$ & Date & $\begin{array}{c}\text { Discharge } \\
\left(\mathrm{ft}^{3} / \mathrm{s}\right)\end{array}$ & Date & $\begin{array}{c}\text { Discharge } \\
\left(\mathrm{ft}^{3} / \mathrm{s}\right)\end{array}$ & Date & $\begin{array}{c}\text { Discharge } \\
\left(\mathrm{ft}^{3} / \mathrm{s}\right)\end{array}$ \\
\hline $\begin{array}{r}10-21-52 \\
1-12-65 \\
7-16-65 \\
11-3-65 \\
3-9-66 \\
5-31-66 \\
11-22-66 \\
2-28-67 \\
5-24-67\end{array}$ & $\begin{array}{l}.12 \\
4.80 \\
.11 \\
.06 \\
.07 \\
0 \\
0 \\
.01\end{array}$ & $\begin{array}{r}8-8-67 \\
9-29-67 \\
11-15-67 \\
1-21-68 \\
4-16-68 \\
7-10-68 \\
10-9-68 \\
1-14-69\end{array}$ & $\begin{array}{r}0 \\
.10 \\
.12 \\
.17 \\
.10 \\
.65 \\
1.40 \\
2.80\end{array}$ & $\begin{array}{r}5-21-69 \\
8-\quad 1-69 \\
12-22-69 \\
2-17-70 \\
5-12-70 \\
8-11-70 \\
11-6-70 \\
3-23-71\end{array}$ & $\begin{array}{l}4.50 \\
.21 \\
1.10 \\
1.20 \\
3.50 \\
0 \\
0 \\
.09\end{array}$ & $\begin{array}{r}6-24-71 \\
8-20-71 \\
11-11-71 \\
1-25-72 \\
4-17-72 \\
7-10-72 \\
12-6-72 \\
5-22-73 \\
6-12-73\end{array}$ & $\begin{array}{l}0.01 \\
.05 \\
.14 \\
.05 \\
0 \\
0 \\
1.0 \\
.81\end{array}$ \\
\hline
\end{tabular}

Deer Creek near Thomas, Oklahoma, Miscellaneous site.

Lat. $35^{\circ} 38^{\prime} 32^{\prime \prime}$, long. $98^{\circ} 44^{\prime} 17^{\prime \prime}$, SE $1 / 4 \mathrm{SW} 1 / 4 \mathrm{sec} .31, \mathrm{~T} .14 \mathrm{~N} ., \mathrm{R} .14 \mathrm{~W}$, at county highway bridge, 7 miles southeast of Thomas, Okia.

$\begin{array}{lr}\text { Date } & \begin{array}{r}\text { Discharge } \\ \left(\mathrm{ft}^{3} / \mathrm{s}\right)\end{array} \\ 9-15-54 & .29\end{array}$

Delaware Creek near Anadrako, Oklahoma, Miscellaneous site.

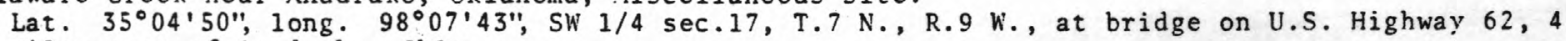
miles east of Anadrako, Okla.

$$
\begin{array}{cr}
\text { Date } & \begin{array}{r}
\text { Discharge } \\
\left(\mathrm{ft}^{3} / \mathrm{s}\right)
\end{array} \\
7-10-52 & .05
\end{array}
$$

Delaware Creek near Wapanucka, Oklahoma, 07334440 , partial record site. Lat. $34^{\circ} 24^{\prime} 30^{\prime \prime}$, long. $96^{\circ} 25^{\prime} 15^{\prime \prime}$, in SW 1/4 sec.1, T.2 S., R. 8 E., Johnston County, at bridge on State Highway 48,2 miles north of Wapanucka, Okla.

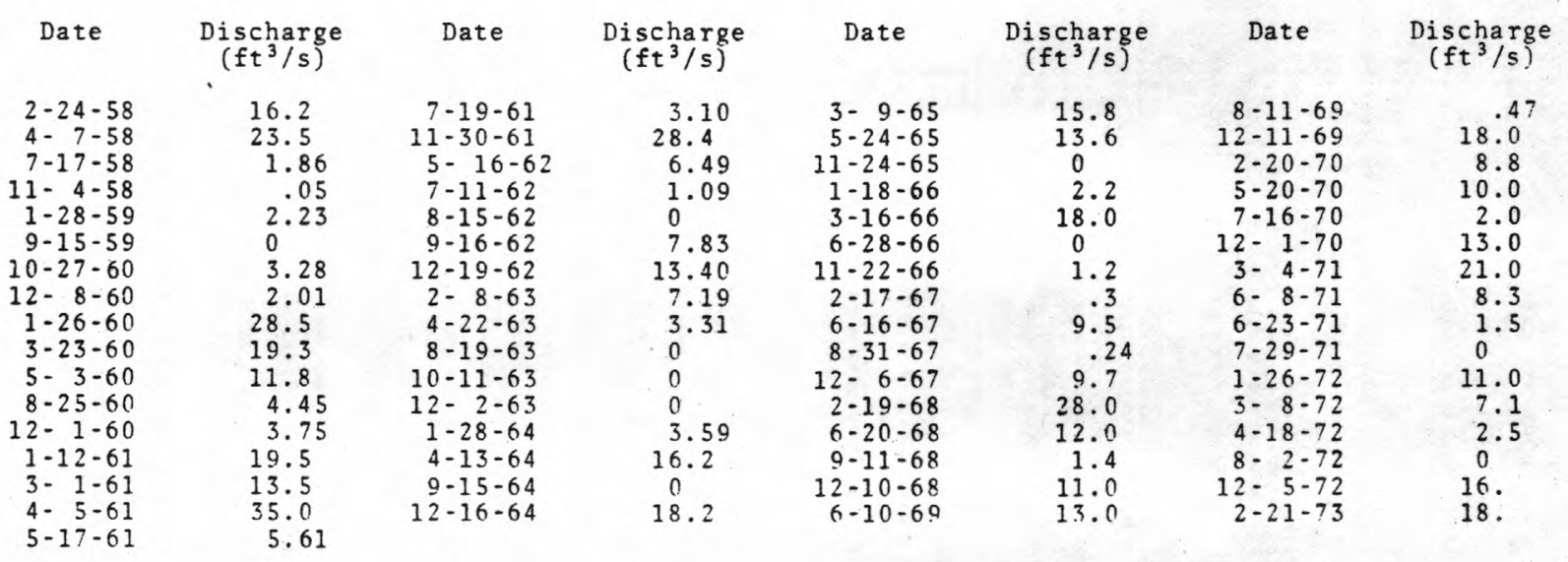


Double Creek near Ramona, Oklahoma, Miscellaneous site.

Lat. $36^{\circ} 32^{\prime} 00^{\prime \prime}$, long. $95^{\circ} 53^{\prime} 00^{\prime \prime}$, SW $1 / 4 \mathrm{SW} 1 / 4 \mathrm{sec} .22, \mathrm{~T} .24 \mathrm{~N}$, , R.13 E., at bridge on U.S. Highway $75,1 / 2$ mile north of Ramona, Okla.

\begin{tabular}{|c|c|c|c|c|c|c|c|}
\hline Date & $\begin{array}{c}\text { Discharge } \\
\left(\mathrm{ft}^{3} / \mathrm{s}\right)\end{array}$ & Date & $\begin{array}{c}\text { Discharge } \\
\left(\mathrm{ft}^{3} / \mathrm{s}\right)\end{array}$ & Date & $\begin{array}{c}\text { Discharge } \\
\left(\mathrm{ft}^{3} / \mathrm{s}\right)\end{array}$ & Date & $\begin{array}{c}\text { Di scharge } \\
\left(\mathrm{ft}^{3} / \mathrm{s}\right)\end{array}$ \\
\hline $\begin{array}{r}11-10-53 \\
12-16-53 \\
1-11-54\end{array}$ & $\begin{array}{l}0 \\
0 \\
0\end{array}$ & $\begin{array}{l}2-15-54 \\
4-5-54 \\
4-26-54\end{array}$ & $\begin{array}{l}0 \\
0 \\
0\end{array}$ & $\begin{array}{l}5-10-54 \\
6-2-54 \\
7-6-54\end{array}$ & $\begin{array}{c}0 \\
25.9 \\
0\end{array}$ & $\begin{array}{l}8-2-54 \\
9-9-54\end{array}$ & $\begin{array}{l}0 \\
0\end{array}$ \\
\hline
\end{tabular}

Duck Pond Creek near Clear Lake, Oklahoma, 07234130 , partial record site. Lat. $36^{\circ} 44^{\prime} 50^{\prime \prime}$, long. $100^{\circ} 17^{\circ} 05^{\prime \prime}$, in NW $1 / 4$ sec.8, T.3 N., R.26 E., Beaver County, at county road bridge, 4 miles north of Clear Lake.

\begin{tabular}{|c|c|c|c|c|c|c|c|}
\hline Date & $\begin{array}{c}\text { Discharge } \\
\left(\mathrm{ft}^{3} / \mathrm{s}\right)\end{array}$ & Date & $\begin{array}{c}\text { Discharge } \\
\left(\mathrm{ft}^{3} / \mathrm{s}\right)\end{array}$ & Date & $\begin{array}{c}\text { Discharge } \\
\left(\mathrm{ft}^{3} / \mathrm{s}\right)\end{array}$ & Date & $\begin{array}{c}\text { Discharge } \\
\left(\mathrm{ft}^{3} / \mathrm{s}\right)\end{array}$ \\
\hline $\begin{array}{r}11-23-66 \\
3-1-67 \\
5-12-67 \\
8-28-67 \\
11-28-67 \\
2-20-68 \\
4-10-68\end{array}$ & $\begin{array}{l}0 \\
.63 \\
0 \\
0 \\
0 \\
.35 \\
.40\end{array}$ & $\begin{array}{r}8-14-68 \\
11-20-68 \\
2-6-69 \\
6-26-69 \\
8-6-69 \\
12-17-69 \\
2-\quad 26-70\end{array}$ & $\begin{array}{l}0 \\
2.80 \\
2.40 \\
5.7 \\
0 \\
2.2 \\
2.5\end{array}$ & $\begin{array}{r}5-14-70 \\
7-24-70 \\
11-6-70 \\
3-11-71 \\
5-28-71 \\
7-27-71 \\
9-23-71\end{array}$ & $\begin{array}{l}. .59 \\
0.16 \\
2.10 \\
0 \\
0 \\
0\end{array}$ & $\begin{array}{r}12-15-71 \\
3-8-72 \\
6-2-72 \\
8-18-72 \\
12-20-72 \\
3-20-73 \\
5-24-73\end{array}$ & $\begin{array}{l}3.1 \\
1.4 \\
.12 \\
0 \\
2.4 \\
3.4 \\
2.1\end{array}$ \\
\hline
\end{tabular}

Eagle Chief Creek near Aline, Oklahoma, 07158100.

Lat. $36^{\circ} 30^{\prime} 23^{\prime \prime}$, long. $98^{\circ} 26^{\prime} 24^{\prime \prime}$, in $\mathrm{NW} 1 / 4 \mathrm{sec} .1, \mathrm{~T} .23 \mathrm{~N} ., \mathrm{R} .12$ W., Alfalfa County, at bridge on State Highway $8 \mathrm{~B}$ at Aline.

\begin{tabular}{|c|c|c|c|c|c|c|c|}
\hline Date & $\begin{array}{c}\text { Discharge } \\
\left(\mathrm{ft}^{3} / \mathrm{s}\right)\end{array}$ & Date & $\begin{array}{c}\text { Discharge } \\
\left(\mathrm{ft}^{3} / \mathrm{s}\right)\end{array}$ & Date & $\begin{array}{c}\text { Discharge } \\
\left(\mathrm{ft}^{3} / \mathrm{s}\right)\end{array}$ & Date & $\begin{array}{c}\text { Discharge } \\
\left(\mathrm{ft}^{3} / \mathrm{s}\right)\end{array}$ \\
\hline $\begin{array}{r}10-14-52 \\
11-4-52 \\
12-3-52 \\
1-5-53 \\
2-3-53 \\
3-3-53 \\
4-21-53 \\
5-15-53 \\
7-7-53 \\
9-1-53 \\
10-14-53 \\
11-3-53 \\
4-5-55 \\
1-7-58 \\
2-4-58 \\
5-20-58 \\
9-29-58 \\
10-21-58\end{array}$ & $\begin{array}{r}2.46 \\
2.58 \\
5.31 \\
5.53 \\
3.96 \\
8.85 \\
3.28 \\
24.80 \\
1.71 \\
1.36 \\
1.06 \\
2.40 \\
2.68 \\
5.83 \\
11.80 \\
10.90 \\
5.98 \\
4.47\end{array}$ & $\begin{array}{r}2-5-59 \\
3-24-59 \\
5-20-59 \\
9-14-59 \\
2-2-61 \\
3-1-61 \\
4-19-61 \\
7-18-61 \\
9-6-61 \\
10-18-61 \\
11-30-61 \\
3-7-61 \\
5-10-62 \\
7-12-62 \\
9-19-62 \\
12-18-62 \\
3-13-63\end{array}$ & $\begin{array}{l}9.81 \\
9.31 \\
7.96 \\
2.24 \\
24.3 \\
19.7 \\
35.5 \\
22.1 \\
16.6 \\
13.5 \\
20.2 \\
22.2 \\
11.2 \\
11.1 \\
10.1 \\
12.7 \\
10.6\end{array}$ & $\begin{array}{r}7-23-63 \\
10-15-63 \\
12-3-63 \\
2-11-64 \\
5-26-64 \\
9-3-64 \\
1-19-65 \\
12-7-65 \\
3-10-66 \\
6-28-66 \\
9-9-66 \\
11-29-66 \\
2-1-67 \\
8-9-67 \\
1-30-68 \\
4-23-68 \\
7-17-68\end{array}$ & $\begin{array}{c}18.3 \\
12.1 \\
12.0 \\
11.0 \\
2.24 \\
8.47 \\
12.4 \\
7.9 \\
8.7 \\
1.4 \\
4.5 \\
2.8 \\
3.9 \\
3.2 \\
4.1 \\
5.1 \\
2.1\end{array}$ & $\begin{array}{r}2-13-69 \\
4-16-69 \\
7-9-69 \\
10-22-69 \\
1-13-70 \\
5-19-70 \\
7-27-70 \\
11-11-70 \\
3-24-71 \\
5-5-71 \\
8-18-71 \\
11-17-71 \\
3-3-72 \\
4-19-72 \\
8-1-72 \\
10-25-72 \\
2-28-73 \\
5-23-73\end{array}$ & $\begin{array}{r}3.0 \\
4.0 \\
4.0 \\
6.2 \\
5.7 \\
5.1 \\
2.7 \\
2.0 \\
3.0 \\
2.1 \\
3.2 \\
4.4 \\
3.5 \\
3.7 \\
2.3 \\
25.0 \\
8.0 \\
34.0\end{array}$ \\
\hline
\end{tabular}

Eagle Chief Creek near Carmen, Oklahoma, Miscellaneous site.

Late. $36^{\circ} 35^{\circ} 00^{\circ}$, long. $98^{\circ} 31^{\prime} 00^{\prime \prime}, S^{\prime} 1 / 4$ sec. 5, T. 24 N., R. $12 \mathrm{~W}^{\prime}$, at pridge on State Highway 45 , $2-3 / 4$ miles west of Carmen, Okla.

\begin{tabular}{|c|c|c|c|c|c|c|c|}
\hline Date & $\begin{array}{c}\text { Discharge } \\
\left(\mathrm{ft}^{3} / \mathrm{s}\right)\end{array}$ & Date & $\begin{array}{c}\text { Discharge } \\
\left(\mathrm{ft}^{3} / \mathrm{s}\right)\end{array}$ & Date & $\begin{array}{c}\text { Discharge } \\
\left(\mathrm{ft}^{3} / \mathrm{s}\right)\end{array}$ & Date & $\begin{array}{c}\text { Discharge } \\
\left(\mathrm{ft}^{3} / \mathrm{s}\right)\end{array}$ \\
\hline $\begin{array}{r}9-28-49 \\
11-14-50 \\
10-4-52 \\
12-3-52 \\
1-5-53\end{array}$ & $\begin{array}{l}6.86 \\
3.32 \\
1.95 \\
3.66 \\
3.02\end{array}$ & $\begin{array}{l}2-3-53 \\
3-3-53 \\
4-21-53 \\
5-15-53 \\
7-7-53\end{array}$ & $\begin{array}{r}2.05 \\
4.39 \\
1.93 \\
9.71 \\
.43\end{array}$ & $\begin{array}{r}9-1-53 \\
10-14-53 \\
11-3-53 \\
1-5-54 \\
2-2-54\end{array}$ & $\begin{array}{r}.68 \\
.17 \\
1.34 \\
1.72 \\
1.95\end{array}$ & $\begin{array}{l}3-2-54 \\
4-5-54 \\
4-27-54 \\
9-20-54\end{array}$ & $\begin{array}{r}2.48 \\
1.26 \\
5.80 \\
.08\end{array}$ \\
\hline
\end{tabular}


Eagle Chief Creek near Cleo, Oklahoma, Miscellaneous site.

Lat. $36^{\circ} 24^{\prime} 30^{\prime \prime}$, long. $98^{\circ} 27^{\prime} 00^{\prime \prime}$, NE $1 / 4$ sec.11, T.22 N., R.12 W., at county highway bridge, $1 / 4 \mathrm{mile}$ west of Cleo, Okla.

\begin{tabular}{|c|c|c|c|c|c|c|c|}
\hline Date & $\begin{array}{c}\text { Discharge } \\
\left(\mathrm{ft}^{3} / \mathrm{s}\right)\end{array}$ & Date & $\begin{array}{c}\text { Discharge } \\
\left(\mathrm{ft}^{3} / \mathrm{s}\right)\end{array}$ & Date & $\begin{array}{c}\text { Discharge } \\
\left(\mathrm{ft}^{3} / \mathrm{s}\right)\end{array}$ & Date & $\begin{array}{c}\text { Discharge } \\
\left(\mathrm{ft}^{3} / \mathrm{s}\right)\end{array}$ \\
\hline $\begin{array}{r}4-29-49 \\
7-27-49 \\
9-28-49 \\
3-27-50 \\
11-14-50 \\
10-5-50 \\
11-14-50 \\
9-6-51 \\
10-16-51\end{array}$ & $\begin{array}{l}71.0 \\
29.2 \\
20.9 \\
10.9 \\
15.7 \\
19.1 \\
17.3 \\
22.7 \\
19.9\end{array}$ & $\begin{array}{r}1-9-52 \\
2-5-52 \\
3-5-52 \\
4-14-52 \\
4-23-52 \\
6-5-52 \\
7-21-52 \\
9-3-52 \\
10-14-52\end{array}$ & $\begin{array}{c}25.5 \\
15.3 \\
21.7 \\
5.64 \\
236.0 \\
16.4 \\
8.36 \\
4.64 \\
3.94\end{array}$ & $\begin{array}{r}11-4-52 \\
12-3-52 \\
1-5-53 \\
2-3-53 \\
3-3-53 \\
4-21-53 \\
5-15-53 \\
7-7-53 \\
9-1-53\end{array}$ & $\begin{array}{c}4.84 \\
7.85 \\
7.68 \\
5.90 \\
19.5 \\
5.39 \\
76.0 \\
2.57 \\
2.58\end{array}$ & $\begin{array}{r}10-14-53 \\
11-3-53 \\
1-5-54 \\
2-2-54 \\
3-1-54 \\
4-5-54 \\
4-27-54 \\
9-20-54\end{array}$ & $\begin{array}{r}2.38 \\
4.68 \\
4.99 \\
5.10 \\
3.98 \\
4.38 \\
5.61 \\
.57\end{array}$ \\
\hline
\end{tabular}

Elk Creek near Roosevelt, Oklahoma, Miscellaneous site.

Lat. $34^{\circ} 51^{\prime} 55^{\prime \prime}$, long. $99^{\circ} 07^{\prime} 08^{\prime \prime}$, south line sec.32, T.5 N., R.18 W., at county highway bridge, $5-1 / 2$ miles west of Roosevelt, Okla.

$\begin{array}{lrrr}\text { Date } & \begin{array}{c}\text { Discharge } \\ \left(\mathrm{ft}^{3} / \mathrm{s}\right)\end{array} & \text { Date } & \begin{array}{c}\text { Discharge } \\ \left(\mathrm{ft}^{3} / \mathrm{s}\right)\end{array} \\ 9-14-49 & 7.59 & 8-12-58 & 2.48\end{array}$

Elk Creek near Sentinel, Oklahoma, Miscellaneous site.

Lat. $34^{\circ} 09^{\prime} 00^{\prime \prime}$, long. $99^{\circ} 12^{\prime} 00^{\prime \prime}, \mathrm{NW} 1 / 4 \mathrm{sec} .27, \mathrm{~T} .8 \mathrm{~N} ., \mathrm{R} .19 \mathrm{~W} .$, at county highway bridge, $1 \mathrm{mile}$ southwest of Sentinel, Oklahoma.

\begin{tabular}{|c|c|c|c|}
\hline Date & $\begin{array}{c}\text { Discharge } \\
\left(\mathrm{ft}^{3} / \mathrm{s}\right)\end{array}$ & Date & $\begin{array}{c}\text { Discharge } \\
\left(\mathrm{ft}^{3} / \mathrm{s}\right)\end{array}$ \\
\hline $2-12-50$ & 7.28 & $8-12-58$ & 1.80 \\
\hline
\end{tabular}

Elm Fork Red River near Mangum, Oklahoma, Miscellaneous site.

Lat. $34^{\circ} 56^{\prime} 04^{\prime \prime}$, long. $99^{\circ} 31^{\prime} 58^{\prime \prime}$, NW $1 / 4 \mathrm{sec} .10, \mathrm{~T} .5 \mathrm{~N} .$, R. $22 \mathrm{~K}$., at bridge on U. S. Highway 283 , $2-3 / 4$ miles north of Mangum, Okla.

\begin{tabular}{|c|c|c|c|c|c|c|c|}
\hline Date & $\begin{array}{c}\text { Discharge } \\
\left(\mathrm{ft}^{3} / \mathrm{s}\right)\end{array}$ & Date & $\begin{array}{c}\text { Discharge } \\
\left(\mathrm{ft}^{3} / \mathrm{s}\right)\end{array}$ & Date & $\begin{array}{c}\text { Discharge } \\
\left(\mathrm{ft}^{3} / \mathrm{s}\right)\end{array}$ & Date & $\begin{array}{c}\text { Discharge } \\
\left(\mathrm{ft}^{3} / \mathrm{s}\right)\end{array}$ \\
\hline $\begin{array}{l}2-26-54 \\
3-26-54\end{array}$ & $\begin{array}{l}2.97 \\
3.49\end{array}$ & $11-6-57$ & 28.2 & $10-7-58$ & 3.24 & $2-25-59$ & 6.30 \\
\hline
\end{tabular}

Farm Pond near Miami, Oklahoma, Miscellaneous site.

Lat. $36^{\circ} 51^{\prime} 41^{\prime \prime}$, long. $94^{\circ} 54^{\prime} 00^{\prime \prime}$, NE 1/4, SW $1 / 4 \mathrm{sec} .1, \mathrm{~T} .27 \mathrm{~N} ., \mathrm{R} .22 \mathrm{E} ., 2$ miles southwest of Miami, Okla.

Date Discharge
$\left(\mathrm{ft}^{3} / \mathrm{s}\right)$

$5-18-43 \quad 41$. 
Finn Creek near Story, Oklahoma, 07328300 , partial record site.

Lat $35^{\circ} 51^{\prime} 15^{\prime \prime}$, long. $97^{\circ} 25^{\prime} 30^{\prime \prime}$, in NE 1/4 sec.5, T.4 N., R.2 W., Garvin County, at bridge on State Highway 24,2 miles east of Story.

\begin{tabular}{|c|c|c|c|c|c|c|c|}
\hline Date & $\begin{array}{c}\text { Discharge } \\
\left(\mathrm{ft}^{3} / \mathrm{s}\right)\end{array}$ & Date & $\begin{array}{c}\text { Discharge } \\
\left(\mathrm{ft}^{3} / \mathrm{s}\right)\end{array}$ & Date & $\begin{array}{c}\text { Discharge } \\
\left(\mathrm{ft}^{3} / \mathrm{s}\right)\end{array}$ & Date & $\begin{array}{c}\text { Discharge } \\
\left(\mathrm{ft}^{3} / \mathrm{s}\right)\end{array}$ \\
\hline $\begin{array}{r}9-5-51 \\
10-9-51 \\
11-26-51 \\
1-14-52 \\
3-14-52 \\
4-22-52 \\
5-13-52 \\
7-23-52 \\
9-14-52 \\
10-10-52 \\
11-19-52 \\
12-24-52 \\
2-5-53 \\
3-11-53 \\
4-15-53 \\
6-3-53 \\
6-24-53 \\
8-20-53 \\
10-11-53 \\
11-18-53 \\
1-29-54 \\
2-26-54 \\
4-19-54 \\
5-28-54\end{array}$ & $\begin{array}{c}.06 \\
.08 \\
.70 \\
.50 \\
1.60 \\
32.2 \\
1.00 \\
.68 \\
.05 \\
0.27 \\
.27 \\
.66 \\
.66 \\
1.38 \\
17.4 \\
.27 \\
0.83 \\
0 \\
1.83 \\
2.06 \\
.81 \\
2.04 \\
0\end{array}$ & $\begin{array}{r}7-14-54 \\
8-23-54 \\
9-15-54 \\
11-2-54 \\
12-8-54 \\
2-1-55 \\
2-28-55 \\
6-14-55 \\
7-28-55 \\
9-20-55 \\
11-1-55 \\
1-4-56 \\
2-15-56 \\
3-30-56 \\
5-3-56 \\
7-19-56 \\
8-22-56 \\
10-12-56 \\
11-14-56 \\
11-20-56 \\
2-11-57 \\
3-14-57 \\
8-1-57\end{array}$ & $\begin{array}{r}.43 \\
.04 \\
.07 \\
.18 \\
.45 \\
.51 \\
.47 \\
.82 \\
.27 \\
2.46 \\
.84 \\
1.22 \\
1.63 \\
.82 \\
.63 \\
.13 \\
0 \\
.04 \\
.12 \\
3.12 \\
.39 \\
.06 \\
2.26\end{array}$ & $\begin{array}{r}10-2-57 \\
1-16-58 \\
3-20-58 \\
6-12-58 \\
9-2-58 \\
10-31-58 \\
1-9-59 \\
7-16-59 \\
9-16-59 \\
10-28-59 \\
12-9-59 \\
9-15-60 \\
12-2-60 \\
1-13-61 \\
3-2-61 \\
4-6-61 \\
12-20-61 \\
8-21-62 \\
7-1-63 \\
7-23-63 \\
8-20-63 \\
9-19-63 \\
10-8-63\end{array}$ & $\begin{array}{c}3.42 \\
3.05 \\
4.41 \\
2.42 \\
1.03 \\
1.32 \\
5.13 \\
2.98 \\
.15 \\
5.47 \\
3.77 \\
1.73 \\
6.93 \\
6.54 \\
4.25 \\
19.4 \\
23.7 \\
.91 \\
1.03 \\
.06 \\
.14 \\
1.01 \\
.29\end{array}$ & $\begin{array}{r}10-30-63 \\
3-8-65 \\
7-13-65 \\
12-1-65 \\
3-22-66 \\
6-15-66 \\
1-30-67 \\
5-19-67 \\
8-9-67 \\
1-26-68 \\
4-9-68 \\
9-18-68 \\
12-13-68 \\
3-20-69 \\
6-13-69 \\
11-7-59 \\
3-25-70 \\
5-21-70 \\
8-4-70 \\
8-24-70 \\
11-16-70 \\
1-18-71 \\
4-5-71 \\
7-20-71\end{array}$ & $\begin{array}{r}.01 \\
2.78 \\
.49 \\
.42 \\
.39 \\
.01 \\
.46 \\
.26 \\
.05 \\
1.50 \\
4.80 \\
3.00 \\
15.00 \\
25.00 \\
12.00 \\
3.60 \\
3.40 \\
2.30 \\
0 \\
.54 \\
13.5 \\
5.20 \\
4.60 \\
1.80\end{array}$ \\
\hline
\end{tabular}

Fish Creek near Reed, Oklahoma, Miscellaneous site.

Lat. $34^{\circ} 53^{\prime} 18^{\prime \prime}$, long. $99^{\circ} 42^{\prime} 17^{\prime \prime}, \mathrm{SE} 1 / 4 \mathrm{SE} 1 / 4 \mathrm{sec} .35, \mathrm{~T} .5 \mathrm{~N} .$, R.24 W., at county highway bridge, $2-3 / 4$ miles south of Reed, Okla.

$\begin{array}{lr}\text { Date } & \begin{array}{c}\text { Discharge } \\ \left(\mathrm{ft}^{3} / \mathrm{s}\right)\end{array} \\ 2-10-54 & .16\end{array}$

Fivemile Creek near Eakley, Oklahoma, Misceallaneous site.

Lat. $35^{\circ} 18^{\prime} 00^{\prime \prime}$, long. $98^{\circ} 36^{\prime} 30^{\prime \prime}$, NE $1 / 4 \mathrm{NE} 1 / 4$ sec.31, T.10 N., R.13 W., at county highway bridge 3 miles west of Eakly, Okla.

\begin{tabular}{|c|c|c|c|c|c|c|c|}
\hline Date & $\begin{array}{c}\text { Discharge } \\
\left(\mathrm{ft}^{3} / \mathrm{s}\right)\end{array}$ & Date & $\begin{array}{c}\text { Discharge } \\
\left(\mathrm{ft}^{3} / \mathrm{s}\right)\end{array}$ & Date & $\begin{array}{c}\text { Discharge } \\
\left(\mathrm{ft}^{3} / \mathrm{s}\right)\end{array}$ & Date & $\begin{array}{c}\text { Discharge } \\
\left(\mathrm{ft}^{3} / \mathrm{s}\right)\end{array}$ \\
\hline $\begin{array}{r}11-5-52 \\
4-30-53\end{array}$ & $\begin{array}{r}.70 \\
2.57\end{array}$ & $6-22-53$ & .39 & $9-8-53$ & .11 & $2-15-55$ & 2.74 \\
\hline
\end{tabular}

Gillespie Spring near Connersville, Oklahoma, Miscellaneous site.

Lat. $34^{\circ} 28^{\prime} 2^{\prime \prime}$, long. $96^{\circ} 41^{\prime} 08^{\prime \prime}$, SW $1 / 4 \mathrm{NW} 1 / 4 \mathrm{sec} .22$, T.1 S., R. 6 E., at site 2 miles northwest of Connersville, Okla.

$\begin{array}{cr}\text { Date } & \begin{array}{r}\text { Discharge } \\ \left(\mathrm{ft}^{3} / \mathrm{s}\right)\end{array} \\ 4-20-54 & 2.09\end{array}$


Goff Creek near Guymon, Oklahoma, 07232590 , partial record site.

Lat. $36^{\circ} 45^{\prime}$, long. $101^{\circ} 30^{\prime}$, east line of $\mathrm{NE} 1 / 4 \mathrm{sW} 1 / 4 \mathrm{sec} .1, \mathrm{~T} .3 \mathrm{~N} ., \mathrm{R} .14 \mathrm{E}$. , at bridge on State Highway 64 , about $2-1 / 4$ miles upstream from mouth and 5 miles north of Guymon.

\begin{tabular}{|c|c|c|c|c|c|c|c|}
\hline Date & $\begin{array}{c}\text { Discharge } \\
\left(\mathrm{ft}^{3} / \mathrm{s}\right)\end{array}$ & Date & $\begin{array}{l}\text { Discharge } \\
\left(\mathrm{ft}^{3} / \mathrm{s}\right)\end{array}$ & Date & $\begin{array}{c}\text { Discharge } \\
\left(\mathrm{ft}^{3} / \mathrm{s}\right)\end{array}$ & Date & $\begin{array}{c}\text { Discharge } \\
\left(\mathrm{ft}^{3} / \mathrm{s}\right)\end{array}$ \\
\hline $\begin{array}{r}10-21-64 \\
3-2-65 \\
4-26-65 \\
7-19-65\end{array}$ & $\begin{array}{l}0 \\
0 \\
0 \\
0\end{array}$ & $\begin{array}{r}10-12-65 \\
1-17-66 \\
3-8-66\end{array}$ & $\begin{array}{l}0 \\
0 \\
0\end{array}$ & $\begin{array}{l}4-11-66 \\
7-6-66 \\
1-16-67\end{array}$ & $\begin{array}{l}0 \\
0 \\
0\end{array}$ & $\begin{array}{r}5-2-67 \\
7-24-67 \\
11-27-67 \\
2-19-68\end{array}$ & $\begin{array}{r}0 \\
0 \\
0 \\
0\end{array}$ \\
\hline
\end{tabular}

Goff Creek near Hough, Oklahoma, 07232580 , partial record site.

Lat. $35^{\circ} 45^{\prime} 00^{\prime \prime}$, long. $101^{\circ} 29^{\prime} 00^{\prime \prime}$, in $\mathrm{NW}^{\prime} 1 / \mathrm{r}$ SE $1 / 4 \mathrm{sec} .29$, T.4 N., R.14 E., Texas County, at low water crossing on oil field service road, 9 miles northeast of Hough, Okla.

\begin{tabular}{|c|c|c|c|c|c|c|c|}
\hline Date & $\begin{array}{c}\text { Discharge } \\
\left(\mathrm{ft}^{3} / \mathrm{s}\right)\end{array}$ & Date & $\begin{array}{c}\text { Discharge } \\
\left(\mathrm{ft}^{3} / \mathrm{s}\right)\end{array}$ & Date & $\begin{array}{c}\text { Discharge } \\
\left(\mathrm{ft}^{3} / \mathrm{s}\right)\end{array}$ & Date & $\begin{array}{c}\text { Discharge } \\
\left(\mathrm{ft}^{3} / \mathrm{s}\right)\end{array}$ \\
\hline $\begin{array}{r}2-19-68 \\
5-27-68 \\
8-12-68 \\
12-24-68\end{array}$ & $\begin{array}{r}.52 \\
.07 \\
.03\end{array}$ & $\begin{array}{r}2-25-69 \\
6-20-69 \\
8-4-69 \\
12-3-69\end{array}$ & $\begin{array}{l}.31 \\
0 \\
0\end{array}$ & $\begin{array}{r}3-9-70 \\
5-19-70 \\
7-30-70 \\
11-9-70\end{array}$ & $0_{0}^{.01}$ & $\begin{array}{l}3-1-71 \\
6-15-71 \\
9-3-71\end{array}$ & $0^{.18}$ \\
\hline
\end{tabular}

Golden Creek near Perkins, Oklahoma, Miscellaneous site.

Lat. $35^{\circ} 59^{\prime} 26^{\prime \prime}$, long. $97^{\circ} 02^{\prime} 51^{\prime \prime}$, SE $1 / 4$ sec.35, T. 18 N., R. 2 E., at bridge on State Highway 33 , 1 mile west of Perkins, Okla.

$\begin{array}{cr}\text { Date } & \begin{array}{c}\text { Discharge } \\ \left(\mathrm{ft}^{3} / \mathrm{s}\right)\end{array} \\ 10-23-52 & .32\end{array}$

Greenleaf Creek near Braggs, Oklahoma, Miscellaneous site.

Lat. $35^{\circ} 37^{\prime} 00^{\prime \prime}$, long. $95^{\circ} 10^{\prime} 00^{\prime \prime}$, near center sec.10, T.13 N., R.20 E., just below Greenleaf Lake and $3-1 / 2$ miles southeast of Braggs, Okla.

$\begin{array}{cccccccc}\text { Date } & \begin{array}{c}\text { Discharge } \\ \left(\mathrm{ft}^{3} / \mathrm{s}\right)\end{array} & \text { Date } & \begin{array}{c}\text { Discharge } \\ \left(\mathrm{ft}^{3} / \mathrm{s}\right)\end{array} & \text { Date } & \begin{array}{c}\text { Discharge } \\ \left(\mathrm{ft}^{3} / \mathrm{s}\right)\end{array} & \text { Date } & \begin{array}{c}\text { Discharge } \\ \left(\mathrm{ft}^{3} / \mathrm{s}^{3}\right)\end{array} \\ 11-15-51 & 30.7 & 5-9-52 & 28.7 & 2-16-53 & .28 & 6-29-53 & .52 \\ 12-6-51 & 25.5 & 6-20-52 & 6.94 & 4-9-53 & 176.0 & 1-25-54 & .55 \\ 2-14-52 & 83.8 & 9-5-52 & .13 & 5-25-53 & 20.3 & 6-7-54 & .52\end{array}$

Gypsum Creek near Olustee, Oklahoma, 07299780, partial record site.

Lat. $34^{\circ} 28^{\prime} 45^{\prime \prime}$, long. $99^{\circ} 26^{\prime} 15^{\prime \prime}$, in SW $1 / 4 \mathrm{sec} .21, \mathrm{~T} .1 \mathrm{~S}$., R. 21 W. , Jackson County, at county bridge on FAS road, 6.5 miles south of olustee and about 2.5 miles upstream from mouth.

\begin{tabular}{|c|c|c|c|c|c|c|c|}
\hline Date & $\begin{array}{c}\text { Discharge } \\
\left(\mathrm{ft}^{3} / \mathrm{s}\right)\end{array}$ & Date & $\begin{array}{c}\text { Discharge } \\
\left(\mathrm{ft}^{3} / \mathrm{s}\right)\end{array}$ & Date & $\begin{array}{c}\text { Discharge } \\
\left(\mathrm{ft}^{3} / \mathrm{s}\right)\end{array}$ & Date & $\begin{array}{c}\text { Discharge } \\
\left(\mathrm{ft}^{3} / \mathrm{s}\right)\end{array}$ \\
\hline $\begin{array}{r}2-26-54 \\
3-25-54 \\
8-20-54 \\
9-22-54 \\
10-21-54 \\
12-14-54 \\
1-14-55 \\
2-16-55 \\
4-10-55 \\
7-14-55 \\
9-6-55 \\
1-5-56 \\
2-15-56 \\
3-15-56 \\
4-18-56\end{array}$ & $\begin{array}{l}.81 \\
1.04 \\
.65 \\
1.18 \\
2.27 \\
3.09 \\
3.48 \\
3.94 \\
2.96 \\
4.12 \\
3.52 \\
5.16 \\
5.51 \\
5.09 \\
2.91\end{array}$ & $\begin{array}{r}6-18-56 \\
7-19-56 \\
9-6-56 \\
10-1-56 \\
10-30-56 \\
12-4-57 \\
1-4-57 \\
2-20-57 \\
5-19-57 \\
4-16-57 \\
6-12-57 \\
7-18-57 \\
3-5-65 \\
6-10-65\end{array}$ & $\begin{array}{r}4.95 \\
1.89 \\
.49 \\
1.99 \\
10.1 \\
3.16 \\
3.49 \\
4.00 \\
2.25 \\
4.00 \\
10.70 \\
7.15 \\
2.15 \\
1.01\end{array}$ & $\begin{array}{r}11-8-65 \\
2-8-66 \\
5-10-66 \\
11-22-66 \\
2-14-67 \\
5-23-67 \\
8-29-67 \\
12-2 S-67 \\
3-26-68 \\
6-24-68 \\
9-13-68 \\
3-26-69 \\
6-11-69 \\
9-9-69\end{array}$ & $\begin{array}{l}5.20 \\
4.7 \\
3.0 \\
1.8 \\
1.5 \\
1.0 \\
1.9 \\
2.0 \\
4.2 \\
2.8 \\
1.3 \\
1.6 \\
3.4 \\
3.6\end{array}$ & $\begin{array}{r}12-8-69 \\
2-16-70 \\
6-10-70 \\
8-6-70 \\
10-7-70 \\
1-20-71 \\
6-3-71 \\
8-4-71 \\
12-21-71 \\
2-17-72 \\
4-12-72 \\
7-19-72 \\
11-6-72 \\
2-8-73\end{array}$ & $\begin{array}{l}2.5 \\
4.3 \\
3.8 \\
.79 \\
1.0 \\
1.3 \\
.02 \\
.24 \\
3.7 \\
2.7 \\
1.2 \\
0 \\
.30 \\
3.30\end{array}$ \\
\hline
\end{tabular}


Hackberry Creek near Sharon, Oklahoma, Miscellaneous site.

Lat. $36^{\circ} 12^{\prime} 00^{\prime \prime}$, long. $99^{\circ} 16^{\prime} 30^{\prime \prime}$, NE $1 / 4$ sec. $24, T .20 \mathrm{~N} ., R^{2} 20$ W., at county highway bridge, 6 miles east of Sharon: also on west line of sec. $34, T .21$ N., R.19 W., at county highway bridge, 6 miles east of Sharon, Oklahoma. Lat. $36^{\circ} 15^{\prime} 00^{\prime \prime}$, long. $99^{\circ} 13^{\prime} 30^{\prime \prime}$.

\begin{tabular}{|c|c|c|c|c|c|c|c|}
\hline Date & $\begin{array}{c}\text { Discharge } \\
\left(\mathrm{ft}^{3} / \mathrm{s}\right)\end{array}$ & Date & $\begin{array}{c}\text { Discharge } \\
\left(\mathrm{ft}^{3} / \mathrm{s}\right)\end{array}$ & Date & $\begin{array}{c}\text { Discharge } \\
\left(\mathrm{ft}^{3} / \mathrm{s}\right)\end{array}$ & Date & $\begin{array}{c}\text { Discharge } \\
\left(\mathrm{ft}^{3} / \mathrm{s}\right)\end{array}$ \\
\hline $\begin{array}{l}4-17-56 \\
5-23-56 \\
6-19-56 \\
4-17-56 \\
5-23-56 \\
6-18-56\end{array}$ & $\begin{array}{l}.04 \\
.03 \\
.004 \\
0 \\
0 \\
0\end{array}$ & $\begin{array}{l}2-27-57 \\
4-11-57 \\
7-10-57 \\
8-14-57 \\
9-12-57\end{array}$ & $\begin{array}{l}.02 \\
.06 \\
.05 \\
.02 \\
.01\end{array}$ & $\begin{array}{l}2-27-57 \\
4-11-57 \\
7-10-57 \\
8-14-57 \\
9-11-57\end{array}$ & $\begin{array}{l}0 \\
0 \\
0 \\
0 \\
0\end{array}$ & $\begin{array}{r}10-18-57 \\
11-13-57 \\
12-20-57 \\
10-17-57 \\
11-13-57 \\
12-19-57\end{array}$ & $\begin{array}{l}.03 \\
.08 \\
.08 \\
0 \\
0 \\
0\end{array}$ \\
\hline
\end{tabular}

Headquarter Creek near Perkins, Oklahoma, Miscellaneous site.

Lat. $35^{\circ} 58^{\prime} 23^{\prime \prime}$, long. $96^{\circ} 57^{\prime} 88^{\prime \prime}$, center of south line of sec.11, T.17 N., R. 3 E., at bridge on county road, 4-1/2 miles east of Vinco and 5-1/4 miles southeast of Perkins, Okia.

Date Discharge

$10-23-52$

Hickory Creek near Marietta, Oklahoma, 07316070 , partial record site.

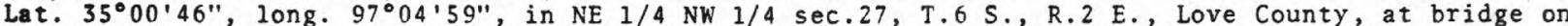
State Highway $77 \mathrm{~S}, 5.5$ miles northeast of Marietta, and 5.5 miles upstream from Lake Texoma.

\begin{tabular}{|c|c|c|c|c|c|c|c|}
\hline Date & $\begin{array}{c}\text { Discharge } \\
\left(\mathrm{ft}^{3} / \mathrm{s}\right)\end{array}$ & Date & $\begin{array}{c}\text { Discharge } \\
\left(\mathrm{ft}^{3} / \mathrm{s}\right)\end{array}$ & Date & $\begin{array}{c}\text { Di scharge } \\
\left(\mathrm{ft}^{3} / \mathrm{s}\right)\end{array}$ & Date & $\begin{array}{c}\text { Discharge } \\
\left(\mathrm{ft}^{3} / \mathrm{s}\right)\end{array}$ \\
\hline $\begin{array}{r}10-29-64 \\
3-10-65 \\
7-14-65 \\
11-29-65 \\
3-22-66 \\
6-14-66 \\
11-2-66 \\
2-21-67 \\
6-7-67\end{array}$ & $\begin{array}{l}.71 \\
4.97 \\
1.41 \\
.68 \\
2.70 \\
.59 \\
1.0 \\
1.2\end{array}$ & $\begin{array}{r}8-8-67 \\
11-16-67 \\
2-20-68 \\
4-25-68 \\
9-18-68 \\
12-18-68 \\
4-3-69 \\
6-5-69\end{array}$ & $\begin{array}{r}.13 \\
1.7 \\
18.0 \\
21.0 \\
0 \\
3.6 \\
20.0 \\
9.7\end{array}$ & $\begin{array}{r}9-16-69 \\
11-20-69 \\
2-19-70 \\
5-13-70 \\
7-17-70 \\
11-19-70 \\
3-2-71 \\
5-5-71\end{array}$ & $\begin{array}{c}0 \\
1.1 \\
4.9 \\
14.0 \\
.06 \\
6.2 \\
5.0 \\
3.8\end{array}$ & $\begin{array}{r}7-8-71 \\
11-24-71 \\
3-27-72 \\
4-17-72 \\
7-31-72 \\
10-2-72 \\
2-20-73 \\
5-14-73\end{array}$ & $\begin{array}{c}0 \\
2.7 \\
7.5 \\
3.2 \\
0 \\
0 \\
4.8 \\
12.0\end{array}$ \\
\hline
\end{tabular}

Hog Creek near Norman, Oklahoma, Miscellaneous site.

Lat. $35^{\circ} 13^{\prime} 37^{\prime \prime}$, long. $97^{\circ} 13^{\prime} 43^{\prime \prime}$, NW $1 / 4 \mathrm{sec} .29, \mathrm{~T} .9 \mathrm{~N} ., \mathrm{R} .1 \mathrm{E}$, at bridge on State Highway 9,12 miles east of Norman, Okla.

Date
$\left(\mathrm{ft}^{3} / \mathrm{s}\right)$

$10-6-53 \quad 0$

Honey Creek near Davis, Oklahoma, Miscellaneous site.

Lat. $34^{\circ} 26^{\prime} 30^{\prime \prime}$, long. $97^{\circ} 09^{\circ} 00^{\prime \prime}$, SE $1 / 4$ sec.25, T.1 S., R.1 E., at pool below Turner Falls 4 miles south of Davis, Oklahoma.

Date

$11-30-49$

$10-10-52$

$\begin{array}{rl}\begin{array}{c}\text { Discharge } \\ \left(\mathrm{ft}^{3} / \mathrm{s}\right)\end{array} & \text { Date } \\ 5.10 & 9-23-53 \\ 1.13 & 9-15-53\end{array}$

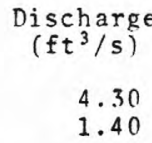

2. 15

11- $1-55$

$5-23-56$
Nate

$8 \cdot 22-56$
Discharge

$\left(\mathrm{ft}^{3} / \mathrm{s}\right)$

1.40
.53 
LOW-FLOW MEASUREMENTS --Continued.

Horse Creek at Afton, Oklahoma, 07189700, partial record site.

Lat. $36^{\circ} 49^{\prime} 50^{\prime \prime}$, long. $94^{\circ} 57^{\prime} 20^{\prime \prime}$, in $\mathrm{NE} 1 / 4 \mathrm{NW} 1 / 4 \mathrm{sec} .33$, T. $26 \mathrm{~N}$, , R.22 E., Ottawa Xounty, at bridge on U.S. Highway 66, at northeast edge of Afton, and about 3 miles upstream from Lake $0^{\prime}$ the Cherokees

\begin{tabular}{|c|c|c|c|c|c|c|c|}
\hline Date & $\begin{array}{c}\text { Discharge } \\
\left(\mathrm{ft}^{3} / \mathrm{s}\right)\end{array}$ & Date & $\begin{array}{c}\text { Discharge } \\
\left(\mathrm{ft}^{3} / \mathrm{s}\right)\end{array}$ & Date & $\begin{array}{c}\text { Discharge } \\
\left(\mathrm{ft}^{3} / \mathrm{s}\right)\end{array}$ & Date & $\begin{array}{c}\text { Discharge } \\
\left(\mathrm{ft}^{3} / \mathrm{s}\right)\end{array}$ \\
\hline $\begin{array}{r}12-21-64 \\
3-22-65 \\
7-13-65 \\
9-16-65 \\
11-23-65 \\
11-30-66 \\
1-19-67 \\
1-31-67\end{array}$ & $\begin{array}{l}.43 \\
.30 \\
.17 \\
.83 \\
.10 \\
0 \\
0 \\
.21\end{array}$ & $\begin{array}{r}5-17-67 \\
8-28-67 \\
11-16-67 \\
3-6-68 \\
6-24-68 \\
8-19-68 \\
10-21-68 \\
1-8-69\end{array}$ & $\begin{array}{r}1.30 \\
0 \\
.48 \\
.36 \\
.10 \\
3.60 \\
.05 \\
1.30\end{array}$ & $\begin{array}{r}5-14-69 \\
9-10-69 \\
1-28-69 \\
4-14-70 \\
8-18-70 \\
11-24-70 \\
1-21-71 \\
6-21-71\end{array}$ & $\begin{array}{l}0.12 \\
4.9 \\
.34 \\
0 \\
1.50 \\
.47 \\
.04\end{array}$ & $\begin{array}{r}8-17-71 \\
11-16-71 \\
2-9-72 \\
5-23-72 \\
8-9-72 \\
6-25-73\end{array}$ & $\begin{array}{r}0 \\
.25 \\
.67 \\
.18 \\
0.16 \\
.16\end{array}$ \\
\hline
\end{tabular}

House Creek near Terleton, Oklahoma, Miscellaneous site.

Lat. $36^{\circ} 16^{\prime} 30^{\prime \prime}$, long. $96^{\circ} 30^{\prime} 00^{\prime \prime}$, NE $1 / 4 \mathrm{NE} 1 / 4$ sec. 25, T. $20 \mathrm{~N} .$, R. 7 E., $1 / 4$ mile downstream from county highway bridge, $1 / 2$ mile west of Terleton, Okla.

$\begin{array}{cccccccc}\text { Date } & \begin{array}{c}\text { Discharge } \\ \left(\mathrm{ft}^{3} / \mathrm{s}\right)\end{array} & \text { Date } & \begin{array}{c}\text { Discharge } \\ \left(\mathrm{ft}^{3} / \mathrm{s}\right)\end{array} & \text { Date } & \begin{array}{c}\text { Discharge } \\ \left(\mathrm{ft}^{3} / \mathrm{s}\right)\end{array} & \begin{array}{c}\text { Date } \\ \left(\mathrm{ft}^{3} / \mathrm{s}\right)\end{array} \\ 11-24-53 & .01 & 5-18-54 & .05 & 10-13-54 & 0 & 3-7-55 & 0 \\ 12-28-53 & .01 & 6-3-54 & .47 & 11-3-54 & 0 & 4-4-55 & 0 \\ 1-11-54 & .01 & 7-6-54 & 0 & 12-14-54 & 0 & 5-2-55 & 0 \\ 2-15-54 & .01 & 8-2-54 & 0 & 1-3-55 & 0 & 7-12-55 & 0 \\ 3-1-54 & .02 & 9-9-54 & 0 & 1-31-55 & 0 & 9-55 & 0 \\ 4-19-54 & .01 & & & & & 0 & 9-55\end{array}$

Hoyle Creek near Ames, Oklahoma, Miscellaneous site.

Lat. $36^{\circ} 15^{\circ} 00^{\prime \prime}$, long. $98^{\circ} 10^{\circ} 00^{\circ}, \mathrm{SE} 1 / 4$ sec.11, T.21 N., R.9 W., at county highway bridge $1 / 2 \mathrm{mile}$ west of Ames, Okla.

\begin{tabular}{|c|c|c|c|c|c|c|c|}
\hline Date & $\begin{array}{c}\text { Discharge } \\
\left(\mathrm{ft}^{3} / \mathrm{s}\right)\end{array}$ & Date & $\begin{array}{c}\text { Discharge } \\
\left(\mathrm{ft}^{3} / \mathrm{s}\right)\end{array}$ & Date & $\begin{array}{c}\text { Discharge } \\
\left(\mathrm{ft}^{3} / \mathrm{s}\right)\end{array}$ & Date & $\begin{array}{c}\text { Discharge } \\
\left(\mathrm{ft}^{3} / \mathrm{s}\right)\end{array}$ \\
\hline $\begin{array}{r}10-2-51 \\
10-30-51 \\
12-10-51 \\
1-8-52 \\
2-4-52 \\
3-3-52 \\
4-14-52\end{array}$ & $\begin{array}{l}.08 \\
.67 \\
.27 \\
.39 \\
.29 \\
.39 \\
.12\end{array}$ & $\begin{array}{r}4-24-52 \\
6-4-52 \\
7-21-52 \\
9-3-52 \\
10-14-52 \\
11-5-52\end{array}$ & $\begin{array}{l}.44 \\
.10 \\
0 \\
0 \\
0 \\
0\end{array}$ & $\begin{array}{r}12-3-52 \\
1-5-53 \\
2-2-53 \\
3-3-53 \\
4-21-53 \\
5-14-53\end{array}$ & $\begin{array}{l}0 \\
0 \\
.01 \\
.37 \\
.09 \\
.08\end{array}$ & $\begin{array}{l}7-22-53 \\
8-31-53 \\
1-4-54 \\
2-1-54 \\
3-2-54 \\
4-5-54\end{array}$ & $\begin{array}{l}.08 \\
.01 \\
.05 \\
.10 \\
.02\end{array}$ \\
\hline
\end{tabular}

Indian Creek near Sayre, Oklahoma, Miscellaneous site.

Lat. $35^{\circ} 17^{\prime} 04^{\prime \prime}$, long. $99^{\circ} 30^{\prime} 17^{\prime \prime}$, on north line, sec.3, T.9 N., R.22 W., at hridge on State Hig.away $152,6-1 / 2$ miles east of Sayre, Okla.

$\begin{array}{cr}\text { Date } & \begin{array}{r}\text { Discharge } \\ \left(\mathrm{ft}^{3} / \mathrm{s}\right)\end{array} \\ 2-14-57 & .06\end{array}$


Indian Creek near Woodward, Oklahoma, Miscellaneous site.

Lat. $36^{\circ} 21^{\prime} 30^{\prime \prime}$, long. $99^{\circ} 17^{\prime} 45^{\prime \prime}$, NW $1 / 4 \mathrm{NW} 1 / 4$ sec. $25, \mathrm{~T} .22 \mathrm{~N}$. , R. 20 W., $6 \mathrm{miles}$ southeast of Woodward, Okla.

\begin{tabular}{|c|c|c|c|c|c|c|c|}
\hline Date & $\begin{array}{c}\text { Discharge } \\
\left(\mathrm{ft}^{3} / \mathrm{s}\right)\end{array}$ & Date & $\begin{array}{c}\text { Discharge } \\
\left(f t^{3} / s\right)\end{array}$ & Date & $\begin{array}{c}\text { Discharge } \\
\left(f t^{3} / \mathrm{s}\right)\end{array}$ & Date & $\begin{array}{c}\text { Discharge } \\
\left(f t^{3} / s\right)\end{array}$ \\
\hline $\begin{array}{r}2-20-44 \\
11-25-44 \\
9-28-48 \\
9-25-51 \\
10-16-51 \\
12-11-51 \\
2-12-52 \\
4-1-52 \\
4-22-52 \\
6-20-52 \\
7-10-52 \\
8-15-52 \\
12-31-53\end{array}$ & $\begin{array}{l}4.74 \\
4.76 \\
2.86 \\
.67 \\
1.23 \\
3.53 \\
7.46 \\
5.76 \\
5.06 \\
2.16 \\
0 \\
0 \\
3.53\end{array}$ & $\begin{array}{r}4-6-54 \\
9-8-54 \\
12-13-54 \\
2-7-55 \\
3-29-55 \\
12-15-55 \\
3-28-56 \\
3-15-56 \\
4-17-56 \\
5-22-56 \\
6-18-56 \\
3-15-56 \\
4-17-56\end{array}$ & $\begin{array}{l}3.13 \\
0 \\
3.24 \\
3.40 \\
2.82 \\
.81 \\
2.57 \\
2.76 \\
.15 \\
0 \\
0 \\
1.62 \\
0\end{array}$ & $\begin{array}{r}5-22-56 \\
6-18-56 \\
3-15-56 \\
4-17-56 \\
5-23-56 \\
6-19-56 \\
12-12-56 \\
2-26-56 \\
4-12-57 \\
7-10-57 \\
8-14-57 \\
9-11-57 \\
3-13-57\end{array}$ & $\begin{array}{l}0 \\
0 \\
2.84 \\
2.31 \\
.85 \\
.44 \\
1.36 \\
0 \\
3.92 \\
4.63 \\
1.44 \\
2.42 \\
3.00\end{array}$ & $\begin{array}{r}2-26-57 \\
4-12-57 \\
7-11-57 \\
8-14-57 \\
9-11-57 \\
2-26-57 \\
4-12-57 \\
7-11-57 \\
8-14-57 \\
9-11-57 \\
10-18-57 \\
11-13-57 \\
12-19-57\end{array}$ & $\begin{array}{l}2.23 \\
6.48 \\
5.60 \\
.02 \\
0 \\
.41 \\
3.91 \\
4.48 \\
0 \\
0 \\
4.17 \\
4.39 \\
4.54\end{array}$ \\
\hline
\end{tabular}

Irwin Spring near Nebo, Oklahoma, Miscellaneous site.

Lat. $34^{\circ} 23^{\prime} 32^{\prime \prime}$, long. $96^{\circ} 56^{\prime} 51^{\prime \prime}$, SE $1 / 4$ sec.19, T.2 S., R.4 E., 1-3/4 miles east of Nebo, Okla.

Date Discharge

$\left(\mathrm{ft}^{3} / \mathrm{s}\right)$

$12-20-54$

2.35

Is land Bayou near Albany, Oklahoma, 07332250 , partial record site.

Lat. $33^{\circ} 51^{\prime} 25^{\prime \prime}$, long. $96^{\circ} 09^{\prime} 55^{\prime \prime}$, in southeast corner sec.17, T.8 S., R.11 E., Bryan County, at county road bridge, 2 miles south of Aibany, and about 3.5 miles upstream from mouth.

\begin{tabular}{|c|c|c|c|c|c|c|c|}
\hline Date & $\begin{array}{c}\text { Discharge } \\
\left(\mathrm{ft}^{3} / \mathrm{s}\right)\end{array}$ & Date & $\begin{array}{c}\text { Discharge } \\
\left(\mathrm{ft}^{3} / \mathrm{s}\right)\end{array}$ & Date & $\begin{array}{c}\text { Discharge } \\
\left(\mathrm{ft}^{3} / \mathrm{s}\right)\end{array}$ & Date & $\begin{array}{c}\text { Discharge } \\
\left(\mathrm{ft}^{3} / \mathrm{s}\right)\end{array}$ \\
\hline $\begin{array}{r}10-29-64 \\
3-4-65 \\
6-18-65 \\
12-1-65 \\
3-16-66 \\
6-9-66 \\
11-2-66 \\
2-17-67\end{array}$ & $\begin{array}{r}.03 \\
14.7 \\
14.5 \\
.02 \\
.09 \\
.17 \\
.10 \\
.02\end{array}$ & $\begin{array}{r}5-26-67 \\
8-31-67 \\
12-8-67 \\
2-20-68 \\
4-25-68 \\
9-19-68 \\
12-18-68 \\
6-10-69\end{array}$ & $\begin{array}{r}4.1 \\
.07 \\
.07 \\
13.0 \\
40.0 \\
7.8 \\
2.0 \\
4.9\end{array}$ & $\begin{array}{r}8-12-69 \\
12-18-69 \\
3-10-70 \\
5-13-70 \\
7-23-70 \\
12-2-70 \\
3-9-71 \\
6-23-71\end{array}$ & $\begin{array}{r}.42 \\
.14 \\
11.0 \\
5.8 \\
.37 \\
.05 \\
3.0 \\
.02\end{array}$ & $\begin{array}{r}8-31-71 \\
1-26-72 \\
4-18-72 \\
8-2-72 \\
10-4-72 \\
2-21-73 \\
6-25-73\end{array}$ & $\begin{array}{l}.05 \\
2.8 \\
.42 \\
0 \\
0 \\
4.6 \\
8.0\end{array}$ \\
\hline
\end{tabular}

Jackfork Creek near Clayton, Nklahoma, Miscellaneous site.

Lat. $34^{\circ} 36^{\circ} 28^{\prime \prime}$, long. $95^{\circ} 20^{\circ} 17^{\prime \prime}$, NW $1 / 4 \mathrm{sec} .32$, T.2 N., R.19 E., at bridge crossing of U.S. Highway

$2 ? 1,1-1 / 2$ miles northeast of ciayton, Okja.

$\begin{array}{cr}\text { Date } & \begin{array}{r}\text { Discharge } \\ \left(\mathrm{ft}^{3} / \mathrm{s}\right)\end{array} \\ 7-10-60 & 3.94\end{array}$

Kingfisher Creek near Kingfisher, Oklahoma, 07159200 , partial record site.

Lat. $35^{\circ} 50^{\prime} 03^{\prime \prime}$, long. $98^{\circ} 03^{\prime} 5^{\prime \prime}$, in NW $1 / 4 \mathrm{SW} 1 / 4 \mathrm{sec} .28, \mathrm{~T} .16 \mathrm{~N} ., \mathrm{R} .8 \mathrm{~W}$., Kingfisher County, at county road bridge, 7.6 miles west of Kingfisher, and at mile 17.6 .

\begin{tabular}{|c|c|c|c|c|c|c|c|}
\hline Date & $\begin{array}{c}\text { Discharge } \\
\left(\mathrm{ft}^{3} / \mathrm{s}\right)\end{array}$ & Date & $\begin{array}{c}\text { Discharge } \\
\left(\mathrm{ft}^{3} / \mathrm{s}\right)\end{array}$ & Date & $\begin{array}{c}\text { Discharge } \\
\left(\mathrm{ft}^{3} / \mathrm{s}\right)\end{array}$ & Date & $\begin{array}{c}\text { Discharge } \\
\left(\mathrm{ft}^{3} / \mathrm{s}\right)\end{array}$ \\
\hline $\begin{array}{r}11-13-70 \\
1-15-71 \\
4-9-71\end{array}$ & $\begin{array}{l}.25 \\
1.6 \\
1.2\end{array}$ & $\begin{array}{r}7-29-71 \\
9-9-71 \\
11-17-71\end{array}$ & $\begin{array}{r}.28 \\
.33\end{array}$ & $\begin{array}{l}3-20-72 \\
4-18-72 \\
8-1-72\end{array}$ & 1.07 & $\begin{array}{r}10-26-72 \\
3-1-73 \\
5-22-73\end{array}$ & $\begin{array}{l}0 \\
2.2 \\
5.3\end{array}$ \\
\hline
\end{tabular}


Kiowa Creek near Slapout, Oklahoma, 07234200 , partial record site.

Lat. $36^{\circ} 36^{\prime} 55^{\prime \prime}$, long. $100^{\circ} 10^{\prime} 00^{\prime \prime}$, on south line sec.20, T.2 N., R.27 E., Beaver County, at bridge on U.S. Highway 270,3 miles west of Slapout.

\begin{tabular}{|c|c|c|c|c|c|c|c|}
\hline Date & $\begin{array}{c}\text { Discharge } \\
\left(\mathrm{ft}^{3} / \mathrm{s}\right)\end{array}$ & Date & $\begin{array}{c}\text { Discharge } \\
\left(\mathrm{ft}^{3} / \mathrm{s}\right)\end{array}$ & Date & $\begin{array}{c}\text { Discharge } \\
\left(\mathrm{ft}^{3} / \mathrm{s}\right)\end{array}$ & Date & $\begin{array}{c}\text { Discharge } \\
\left(\mathrm{ft}^{3} / \mathrm{s}\right)\end{array}$ \\
\hline $\begin{array}{r}11-24-44 \\
7-7-49 \\
4-11-50 \\
10-8-51 \\
1-7-52 \\
1-28-52 \\
4-17-52 \\
6-19-52 \\
8-11-52 \\
11-24-53 \\
2-18-54 \\
6-11-54 \\
9-8-54 \\
11-17-54 \\
1-24-55 \\
3-23-55 \\
8-3-55 \\
10-19-55 \\
3-28-56 \\
6-11-56 \\
12-20-56\end{array}$ & $\begin{array}{c}4.4 \\
11.5 \\
9.21 \\
7.33 \\
13.6 \\
12.9 \\
11.4 \\
3.31 \\
1.96 \\
9.02 \\
9.16 \\
3.93 \\
.05 \\
4.63 \\
10.1 \\
7.82 \\
5.32 \\
5.92 \\
8.45 \\
8.77 \\
7.10\end{array}$ & $\begin{array}{r}2-11-57 \\
9-24-57 \\
10-30-57 \\
12-2-57 \\
3-27-58 \\
7-25-58 \\
10-31-58 \\
2-20-59 \\
4-20-59 \\
7-14-59 \\
9-2-59 \\
9-30-59 \\
10-29-59 \\
12-9-59 \\
1-6-60 \\
2-19-60 \\
5-27-60 \\
6-27-60 \\
8-22-60 \\
10-24-60 \\
1-6-61\end{array}$ & $\begin{array}{r}7.66 \\
6.46 \\
7.38 \\
8.97 \\
9.47 \\
6.59 \\
5.62 \\
9.56 \\
7.90 \\
3.28 \\
1.13 \\
2.43 \\
5.19 \\
5.59 \\
10.4 \\
8.38 \\
3.46 \\
2.35 \\
6.37 \\
6.62 \\
6.37\end{array}$ & $\begin{array}{r}4-20-61 \\
8-3-61 \\
10-19-62 \\
7-25-63 \\
10-17-63 \\
12-5-63 \\
9-1-64 \\
3-2-65 \\
4-27-65 \\
7-7-65 \\
10-8-65 \\
2-15-66 \\
3-8-66 \\
4-26-66 \\
7-6-66 \\
10-7-66 \\
1-17-67 \\
5-12-67 \\
8-28-67 \\
11-28-67 \\
2-6-68\end{array}$ & $\begin{array}{r}8.85 \\
5.23 \\
2.05 \\
4.04 \\
21.1 \\
6.79 \\
.79 \\
6.76 \\
8.20 \\
4.35 \\
4.00 \\
12.00 \\
8.7 \\
7.1 \\
2.1 \\
5.4 \\
7.3 \\
4.0 \\
2.8 \\
3.1 \\
5.2\end{array}$ & $\begin{array}{r}4-10-68 \\
8-9-68 \\
11-20-68 \\
2-6-69 \\
6-26-69 \\
8-6-69 \\
12-17-69 \\
2-26-70 \\
4-16-70 \\
7-24-70 \\
11-6-70 \\
3-11-71 \\
5-28-71 \\
9-9-71 \\
11-11-71 \\
3-3-72 \\
6-2-72 \\
9-7-72 \\
12-21-72 \\
3-20-73 \\
5-24-73\end{array}$ & $\begin{array}{l}4.7 \\
2.0 \\
7.4 \\
7.0 \\
2.3 \\
.44 \\
7.0 \\
6.4 \\
7.7 \\
1.2 \\
4.4 \\
6.6 \\
1.7 \\
.11 \\
2.7 \\
5.9 \\
3.6 \\
9.5 \\
6.8 \\
10 . \\
10 .\end{array}$ \\
\hline
\end{tabular}

Kizer Creek near Mutual, Oklahoma, Miscellaneous site.

Lat. $36^{\circ} 10^{\prime} 00^{\prime \prime}$, long. $99^{\circ} 07^{\prime} 30^{\prime \prime}$, SW $1 / 4 \mathrm{sec} .33$, T.20 N., R.18 w., at county highway bridge, 5-1/2 miles southeast of Mutual, okla.

\begin{tabular}{|c|c|c|c|c|c|c|c|}
\hline Date & $\begin{array}{c}\text { Discharge } \\
\left(\mathrm{ft}^{3} / \mathrm{s}\right)\end{array}$ & Date & $\begin{array}{c}\text { Discharge } \\
\left(\mathrm{ft}^{3} / \mathrm{s}\right)\end{array}$ & Date & $\begin{array}{c}\text { Discharge } \\
\left(\mathrm{ft}^{3} / \mathrm{s}\right)\end{array}$ & Date & $\begin{array}{c}\text { Discharge } \\
\left(\mathrm{ft}^{3} / \mathrm{s}\right)\end{array}$ \\
\hline $\begin{array}{l}3-16-56 \\
4-17-56 \\
5-22-56\end{array}$ & $0^{.34}$ & $\begin{array}{l}6-19-56 \\
2-17-57 \\
4-11-57\end{array}$ & $\begin{array}{l}0 \\
.24 \\
.49\end{array}$ & $\begin{array}{l}7-10-57 \\
8-14-57 \\
9-12-57\end{array}$ & $0^{.47}$ & $\begin{array}{l}10-18-57 \\
11-13-57 \\
12-19-57\end{array}$ & $\begin{array}{l}.10 \\
.29 \\
.44\end{array}$ \\
\hline
\end{tabular}

Lake Creek near Swan Lake, Oklahoma, Miscellaneous site.

Lat. $35^{\circ} 15^{\prime} 00^{\prime \prime}$, long. $98^{\circ} 32^{\prime} 00^{\prime \prime}$, center of south line of sec.13, T.9 N., R.13 W., at bridge on county road, 1 mile northwest of Swan Lake, Okla.

\begin{tabular}{|c|c|c|c|c|c|c|c|}
\hline Date & $\begin{array}{c}\text { Discharge } \\
\left(\mathrm{ft}^{3} / \mathrm{s}\right)\end{array}$ & Date & $\begin{array}{c}\text { Discharge } \\
\left(\mathrm{ft}^{3} / \mathrm{s}\right)\end{array}$ & Date & $\begin{array}{c}\text { Discharge } \\
\left(\mathrm{ft}^{3} / \mathrm{s}\right)\end{array}$ & Date & $\begin{array}{c}\text { Discharge } \\
\left(\mathrm{ft}^{3} / \mathrm{s}\right)\end{array}$ \\
\hline $\begin{array}{r}10-5-52 \\
4-3-53\end{array}$ & $\begin{array}{l}0 \\
3.20\end{array}$ & $6-22-53$ & 0 & $9-8-53$ & 0 & $2-15-55$ & 1.85 \\
\hline
\end{tabular}

Lawrence Spring near Sulfur, Oklahoma, Miscellaneous site.
Lat. $34^{\circ} 27^{\prime} 30^{\prime \prime}$, long. $96^{\circ} 2^{\prime} 00^{\prime \prime}, \mathrm{N} 1 / 4$ sec. $24, \mathrm{~T}, 1$ S., R. 3 E., 4 miles southeast of Sulphur, Ok1a.

\begin{tabular}{|c|c|c|c|c|c|c|c|}
\hline Date & $\begin{array}{c}\text { Discharge } \\
\left(\mathrm{ft}^{3} / \mathrm{s}\right)\end{array}$ & Date & $\begin{array}{c}\text { Discharge } \\
\left(\mathrm{ft}^{3} / \mathrm{s}\right)\end{array}$ & Date & $\begin{array}{c}\text { Discharge } \\
\left(\mathrm{ft}^{3} / \mathrm{s}\right)\end{array}$ & Date & $\begin{array}{l}\text { Discharge } \\
\left(\mathrm{ft}^{3} / \mathrm{s}\right)\end{array}$ \\
\hline $\begin{array}{l}3-23-49 \\
7-19-49 \\
3-5-52\end{array}$ & $\begin{array}{l}4.85 \\
3.91 \\
4.27\end{array}$ & $\begin{array}{r}10-9-52 \\
3-18-53 \\
6-23-53\end{array}$ & $\begin{array}{l}3.53 \\
3.28 \\
4.74\end{array}$ & $\begin{array}{r}9-24-53 \\
9-15-53 \\
12-20-54\end{array}$ & $\begin{array}{l}2.74 \\
1.67 \\
2.40\end{array}$ & $\begin{array}{l}5-22-56 \\
9-\quad 6-56\end{array}$ & $\begin{array}{r}.22 \\
1.40\end{array}$ \\
\hline
\end{tabular}


Leader Creek at Tupelo, Oklahoma, 07334420 , partial record site.

Lat. $34^{\circ} 35^{\prime} 55^{\prime \prime}$, long. $96^{\circ} 23^{\prime} 45^{\prime \prime}$, in NW $1 / 4 \mathrm{sec} .31, \mathrm{~T} .2 \mathrm{~N} .$, R.9 E., Coal County, at bridge on State Highway 3, 1 mile east of Tupelo.

\begin{tabular}{|c|c|c|c|c|c|c|c|}
\hline Date & $\begin{array}{c}\text { Discharge } \\
\left(\mathrm{ft}^{3} / \mathrm{s}\right)\end{array}$ & Date & $\begin{array}{c}\text { Discharge } \\
\left(\mathrm{ft}^{3} / \mathrm{s}\right)\end{array}$ & Date & $\begin{array}{c}\text { Di scharge } \\
\left(\mathrm{ft}^{3} / \mathrm{s}\right)\end{array}$ & Date & $\begin{array}{c}\text { Discharge } \\
\left(\mathrm{ft}^{3} / \mathrm{s}\right)\end{array}$ \\
\hline $\begin{array}{r}2-24-58 \\
4-7-58 \\
7-17-58 \\
11-4-58 \\
1-28-59 \\
8-27-59 \\
9-14-59 \\
10-26-59 \\
12-7-59 \\
1-25-60 \\
3-21-60 \\
8-23-60 \\
11-29-60 \\
1-10-61 \\
2-27-61 \\
4-4-61\end{array}$ & $\begin{array}{c}8.41 \\
7.58 \\
.30 \\
.44 \\
.29 \\
.09 \\
0 \\
.41 \\
2.85 \\
18.6 \\
13.4 \\
1.32 \\
0 \\
6.84 \\
4.16 \\
6.70\end{array}$ & $\begin{array}{r}5-15-61 \\
9-11-61 \\
10-31-61 \\
5-16-62 \\
7-11-62 \\
8-15-62 \\
9-19-62 \\
12-12-62 \\
2-8-63 \\
4-22-63 \\
8-19-63 \\
10-11-63 \\
12-12-63 \\
2-17-64 \\
4-13-64 \\
9-15-64\end{array}$ & $\begin{array}{l}1.00 \\
0 \\
2.85 \\
3.52 \\
.03 \\
0 \\
4.70 \\
2.83 \\
2.37 \\
2.75 \\
0 \\
0 \\
0 \\
0 \\
.23\end{array}$ & $\begin{array}{r}12-16-64 \\
3-9-65 \\
5-24-65 \\
12-1-65 \\
3-17-66 \\
6-7-66 \\
11-22-66 \\
2-13-67 \\
6-13-67 \\
8-29-67 \\
12-6-67 \\
2-19-68 \\
6-20-68 \\
9-11-68 \\
12-10-68 \\
6-10-69\end{array}$ & $\begin{array}{c}2.02 \\
4.61 \\
3.16 \\
0 \\
.83 \\
.08 \\
0 \\
0 \\
0.59 \\
0.28 \\
11.0 \\
7.8 \\
.24 \\
8.0 \\
10.0\end{array}$ & $\begin{array}{r}8-11-69 \\
12-11-69 \\
2-20-70 \\
5-20-70 \\
7-16-70 \\
12-1-70 \\
3-4-71 \\
6-23-71 \\
7-29-71 \\
1-26-72 \\
3-8-72 \\
4-18-72 \\
8-2-72 \\
12-5-72 \\
2-21-73 \\
5-15-73\end{array}$ & $\begin{array}{c}.15 \\
3.6 \\
.40 \\
.38 \\
.24 \\
11.0 \\
12.0 \\
.05 \\
0 \\
.27 \\
2.0 \\
2.5 \\
0 \\
.08 \\
3.0 \\
16.0\end{array}$ \\
\hline
\end{tabular}

Leaper Creek near Washita, Oklahoma, Miscellaneous site.

Lat. $35^{\circ} 07^{\prime} 23^{\prime \prime}$, long. $98^{\circ} 19^{\prime} 25^{\prime \prime}$, SW $1 / 4 \mathrm{sec} 35$, T.8 N., R.11 W., at small bridge, $1-1 / 4$ miles northeast of Washita, Okla.

$\begin{array}{lrrr}\text { Date } & \begin{array}{c}\text { Discharge } \\ \left(\mathrm{ft}^{3} / \mathrm{s}\right)\end{array} & \text { Date } & \begin{array}{r}\text { Discharge } \\ \left(\mathrm{ft}^{3} / \mathrm{s}\right)\end{array} \\ 8-1-56 & .62 & 8-30-56 & .28\end{array}$

Lebos Creek near Eldorado, Oklahoma, Miscellaneous site.

Lat. $34^{\circ} 28^{\prime} 30^{\prime \prime}$, long. $99^{\circ} 40^{\prime} 00^{\prime \prime}, \mathrm{SE} 1 / 4 \mathrm{sec} .11$, T.1 S., R. $24 \mathrm{~W}$., at bridge on State Highway $90,1-1 / 2$ miles west of Eldorado, Okla.

\begin{tabular}{|c|c|c|c|c|c|c|c|}
\hline Date & $\begin{array}{c}\text { Discharge } \\
\left(\mathrm{ft}^{3} / \mathrm{s}\right)\end{array}$ & Date & $\begin{array}{c}\text { Discharge } \\
\left(\mathrm{ft}^{3} / \mathrm{s}\right)\end{array}$ & Date & $\begin{array}{c}\text { Discharge } \\
\left(\mathrm{ft}^{3} / \mathrm{s}\right)\end{array}$ & Date & $\begin{array}{c}\text { Discharge } \\
\left(\mathrm{ft}^{3} / \mathrm{s}\right)\end{array}$ \\
\hline $\begin{array}{r}9-24-51 \\
1-15-52 \\
3-17-52 \\
6-10-52 \\
7-24-52 \\
8-26-52\end{array}$ & $\begin{array}{l}8.24 \\
7.35 \\
7.11 \\
5.66 \\
5.04 \\
6.13\end{array}$ & $\begin{array}{r}9-29-52 \\
11-13-52 \\
12-18-52 \\
1-19-53 \\
5-15-53 \\
9-29-53\end{array}$ & $\begin{array}{l}6.70 \\
7.68 \\
8.62 \\
9.16 \\
6.78 \\
4.95\end{array}$ & $\begin{array}{r}11-16-53 \\
12-18-53 \\
3-25-54 \\
8-20-54 \\
9-22-54 \\
10-21-54\end{array}$ & $\begin{array}{r}5.79 \\
9.78 \\
4.04 \\
.98 \\
1.25 \\
3.90\end{array}$ & $\begin{array}{r}12-14-54 \\
1-14-55 \\
2-16-55 \\
4-19-55 \\
7-14-55 \\
9-6-55\end{array}$ & $\begin{array}{l}3.21 \\
3.90 \\
5.63 \\
4.78 \\
5.22 \\
2.82\end{array}$ \\
\hline
\end{tabular}

Lebos Creek near Vinson, Oklahoma, Miscellaneous site.
Lat. $34^{\circ} 51^{\prime} 00^{\circ}$, long. $99^{\circ} 55^{\prime} 00^{\prime \prime}, \mathrm{SE} 1 / 4$ sec.3, T. 4 ., R. 26 W., at bridge on State Highway $30,5 \mathrm{miles}$ southwest of Vinson, okla.

\begin{tabular}{|c|c|c|c|c|c|c|c|}
\hline Date & $\begin{array}{c}\text { Discharge } \\
\left(\mathrm{ft}^{3} / \mathrm{s}\right)\end{array}$ & Date & $\begin{array}{c}\text { Discharge } \\
\left(\mathrm{ft}^{3} / \mathrm{s}\right)\end{array}$ & Date & $\begin{array}{c}\text { Discharge } \\
\left(\mathrm{ft}^{3} / \mathrm{s}\right)\end{array}$ & Date & $\begin{array}{c}\text { Discharge } \\
\left(\mathrm{ft}^{3} / \mathrm{s}\right)\end{array}$ \\
\hline $\begin{array}{l}4-20-51 \\
9-24-51\end{array}$ & $\begin{array}{r}7.26 \\
.04\end{array}$ & $\begin{array}{l}1-15-52 \\
3-17-52\end{array}$ & $\begin{array}{l}31.4 \\
12.0\end{array}$ & $\begin{array}{l}7-24-52 \\
8-20-52\end{array}$ & $\begin{array}{l}0 \\
0\end{array}$ & $9-29-52$ & 0 \\
\hline
\end{tabular}

Lee Creek near Short, Oklahoma, 07249800 , partial record site.

Lat. $35^{\circ} 30^{\prime} 00^{\prime \prime}$, long. $94^{\circ} 30^{\prime} 00^{\prime \prime}$, on line between secs.27 and 34, T.13 N., R.26 E., at highway bridge, 2.0 miles west os Short, Okla.

\begin{tabular}{|c|c|c|c|c|c|c|c|}
\hline Date & $\begin{array}{c}\text { Discharge } \\
\left(\mathrm{ft}^{3} / \mathrm{s}\right)\end{array}$ & Date & $\begin{array}{c}\text { Discharge } \\
\left(\mathrm{ft}^{3} / \mathrm{s}\right)\end{array}$ & Date & $\begin{array}{c}\text { Nischarge } \\
\left(\mathrm{ft}^{3} / \mathrm{s}\right)\end{array}$ & Date & $\begin{array}{c}\text { Discharge } \\
\left(\mathrm{ft}^{3} / \mathrm{s}\right)\end{array}$ \\
\hline $\begin{array}{r}6-18-58 \\
8-27-58 \\
10-8-58 \\
11-5-58 \\
7-1-59\end{array}$ & $\begin{array}{l}35.1 \\
42.9 \\
51.7 \\
19.9 \\
22.7\end{array}$ & $\begin{array}{l}8-17-59 \\
7-14-60 \\
8-17-60 \\
9-27-60\end{array}$ & $\begin{array}{r}7.6 \\
8.72 \\
18.40 \\
3.30\end{array}$ & $\begin{array}{l}7-11-60 \\
7-12-61 \\
9-26-61 \\
5-24-62\end{array}$ & $\begin{array}{r}11.50 \\
7.22 \\
47.9 \\
27.3\end{array}$ & $\begin{array}{l}6-20-62 \\
7-18-62 \\
8-15-62 \\
4-18-63 \\
7-29-63\end{array}$ & $\begin{array}{c}16.1 \\
9.60 \\
7.58 \\
30.8 \\
1.37\end{array}$ \\
\hline
\end{tabular}


Lick Creek near Unger, Oklahoma, Miscellaneous site.

Lat. $33^{\circ} 57^{\prime} 55^{\prime \prime}$, long. $95^{\circ} 48^{\prime} 17^{\prime \prime}$, in SW $1 / 4 \mathrm{SW} 1 / 4 \mathrm{sec} .7, \mathrm{~T} .7 \mathrm{~S} ., \mathrm{R} .15 \mathrm{E}$. , at county highway bridge

5 miles south of Unger, okla.

Date Discharge

$$
\left(\mathrm{ft}^{3} / \mathrm{s}\right)
$$

$12-12-57$

8.80

Little Deep Fork near Edna, Ok lahoma, Miscellaneous site.

Lat. $35^{\circ} 40^{\circ} 00^{\prime \prime}$, long. $96^{\circ} 10^{\prime} 00^{\prime \prime}$, SW $1 / 4 \mathrm{sec} .12, \mathrm{~T} .14 \mathrm{~N} ., \mathrm{R} .10 \mathrm{E}$. , at county highway bridge, $1 / 4 \mathrm{mile}$ south of Edna, Okla.

\begin{tabular}{|c|c|c|c|c|c|c|c|}
\hline Date & $\begin{array}{c}\text { Di scharge } \\
\left(\mathrm{ft}^{3} / \mathrm{s}\right)\end{array}$ & Date & $\begin{array}{c}\text { Discharge } \\
\left(\mathrm{ft}^{3} / \mathrm{s}\right)\end{array}$ & Date & $\begin{array}{c}\text { nischarge } \\
\left(\mathrm{ft}^{3} / \mathrm{s}\right)\end{array}$ & Date & $\begin{array}{c}\text { Discharge } \\
\left(\mathrm{ft}^{3} / \mathrm{s}\right)\end{array}$ \\
\hline $\begin{array}{r}9-18-51 \\
10-20-51 \\
12-12-51 \\
1-15-52 \\
2-27-52 \\
4-24-52 \\
6-3-52 \\
7-14-52 \\
9-3-52 \\
10-7-53 \\
11-17-53\end{array}$ & $\begin{array}{r}16.8 \\
209.0 \\
13.4 \\
19.8 \\
64.3 \\
271.0 \\
37.5 \\
3.93 \\
.20 \\
3.25 \\
.67\end{array}$ & $\begin{array}{r}12-28-53 \\
12-29-53 \\
2-1-54 \\
3-9-54 \\
4-19-54 \\
6-1-54 \\
7-12-54 \\
8-20-54 \\
10-5-54 \\
11-18-54\end{array}$ & $\begin{array}{c}2.74 \\
2.17 \\
3.87 \\
3.65 \\
2.02 \\
11.6 \\
.04 \\
.05 \\
0 \\
0\end{array}$ & $\begin{array}{r}1-17-55 \\
3-2-55 \\
4-11-55 \\
5-31-55 \\
7-11-55 \\
10-11-55 \\
11-21-55 \\
1-6-56 \\
1-21-56 \\
2-29-56\end{array}$ & $\begin{array}{l}.99 \\
.95 \\
59.9 \\
12.7 \\
0 \\
1.78 \\
0 \\
0 \\
0 \\
.10\end{array}$ & $\begin{array}{r}4-10-56 \\
5-30-56 \\
7-13-56 \\
8-27-56 \\
11-14-56 \\
1-3-57 \\
2-6-57 \\
3-25-57 \\
7-9-57 \\
9-5-57\end{array}$ & $\begin{array}{l}.20 \\
7.64 \\
0 \\
0 \\
.02 \\
.05 \\
1.16 \\
7.42 \\
33.9 \\
1.51\end{array}$ \\
\hline
\end{tabular}

Little Deer Creek near Thomas, Oklahoma, Miscellaneous site.

Lat. $35^{\circ} 43^{\prime} 09^{\prime \prime}$, long. $98^{\circ} 46^{\prime} 17^{\prime \prime}$, SW $1 / 4 \mathrm{SE} 1 / 4$ sec.2, T.i4 N., R.15 w., at county highway bridge,

2 miles southwest of Thomas, Okia.

$\begin{array}{lr}\text { Date } & \begin{array}{r}\text { Discharge } \\ \left(\mathrm{ft}^{3} / \mathrm{s}\right)\end{array} \\ 9-15-54 & .10\end{array}$

Little East Blue near Pontotoc, Oklahoma, Miscellaneous site.

Lat. $34^{\circ} 29^{\prime} 32^{\prime \prime}$, long. $96^{\circ} 38^{\circ} 00^{\prime \prime}$, NW $1 / 4 \mathrm{sec} 12$, T.I S., R.6 E., just west of State Highway 99 in Pontotoc, Okla.

$\begin{array}{cr}\text { Date } & \begin{array}{r}\text { Discharge } \\ \left(\mathrm{ft}^{3} / \mathrm{s}\right)\end{array} \\ 4-20-54 & .30\end{array}$

Little Lee Creek near Short, Oklahoma, 07249900 , partial record site.

Lat. $35^{\circ} 30^{\prime} 00^{\prime \prime}$, long. $94^{\circ} 30^{\circ} 00^{\prime \prime}$, in $\mathrm{NW} 1 / \mathrm{r}$ sec. 28 , T. $13 \mathrm{~N}$., R.26 E., at highway bridge $2-1 / 4 \mathrm{miles}$ upstream from mouth and 3.0 miles west of Short, Okla.

\begin{tabular}{|c|c|c|c|c|c|c|c|}
\hline Date & $\begin{array}{c}\text { Discharge } \\
\left(\mathrm{ft}^{3} / \mathrm{s}\right)\end{array}$ & Date & $\begin{array}{c}\text { Discharge } \\
\left(\mathrm{ft}^{3} / \mathrm{s}\right)\end{array}$ & Date & $\begin{array}{c}\text { Discharge } \\
\left(\mathrm{ft}^{3} / \mathrm{s}\right)\end{array}$ & Date & $\begin{array}{c}\text { Discharge } \\
\left(\mathrm{ft}^{3} / \mathrm{s}\right)\end{array}$ \\
\hline $\begin{array}{r}6-18-58 \\
8-27-58 \\
10-8-58 \\
11-5-58 \\
7-1-59 \\
8-17-59\end{array}$ & $\begin{array}{l}10.6 \\
10.4 \\
12.3 \\
7.84 \\
8.30 \\
2.21\end{array}$ & $\begin{array}{l}7-14-60 \\
8-17-60 \\
9-27-60 \\
7-11-60 \\
7-12-61\end{array}$ & $\begin{array}{r}2.31 \\
15.40 \\
1.10 \\
6.02 \\
1.84\end{array}$ & $\begin{array}{l}9-26-61 \\
5-24-62 \\
6-20-62 \\
7-18-62 \\
8-15-62\end{array}$ & $\begin{array}{c}15.6 \\
16.0 \\
8.97 \\
8.92 \\
1.12\end{array}$ & $\begin{array}{r}4-18-63 \\
7-29-63 \\
11-26-63 \\
7-6-64 \\
11-16-64 \\
8-9-65\end{array}$ & $\begin{array}{l}9.95 \\
0 \\
0 \\
1.09 \\
1.11 \\
0\end{array}$ \\
\hline
\end{tabular}


Little Monument Creek near Clinton, Oklahoma, Miscellaneous site.

Lat. $35^{\circ} 24^{\prime} 00^{\prime \prime}$, long. $99^{\circ} 13^{\prime} 00^{\prime \prime}$, SW $1 / 4 \mathrm{sec} .21$, T.11 N., R.19 W., at county highway bridge, 4 miles southwest of Foss and 16 miles southwest of $\mathrm{Cl}$ inton, Okla.

\begin{tabular}{|c|c|c|c|c|c|c|c|}
\hline Date & $\begin{array}{c}\text { Discharge } \\
\left(\mathrm{ft}^{3} / \mathrm{s}\right)\end{array}$ & Date & $\begin{array}{c}\text { Discharge } \\
\left(\mathrm{ft}^{3} / \mathrm{s}\right)\end{array}$ & Date & $\begin{array}{c}\text { Discharge } \\
\left(\mathrm{ft}^{3} / \mathrm{s}\right)\end{array}$ & Date & $\begin{array}{c}\text { Discharge } \\
\left(\mathrm{ft}^{3} / \mathrm{s}\right)\end{array}$ \\
\hline $\begin{array}{r}4-27-54 \\
6-29-54 \\
12-1-54\end{array}$ & $\begin{array}{l}.62 \\
.07 \\
.51\end{array}$ & $\begin{array}{l}1-17-55 \\
1-24-55\end{array}$ & $\begin{array}{l}.74 \\
.79\end{array}$ & $\begin{array}{l}3-1-55 \\
4-19-55\end{array}$ & $\begin{array}{l}.73 \\
.64\end{array}$ & $\begin{array}{l}3-20-56 \\
9-10-56 \\
9-17-56\end{array}$ & $0^{.81}$ \\
\hline
\end{tabular}

Little Spring Creek near Locust Grove, Oklahoma, Miscellaneous site.

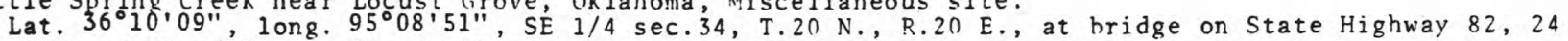

miles south of Locust Grove, Okia.

$\begin{array}{cr}\text { Date } & \begin{array}{r}\text { Discharge } \\ \left(\mathrm{ft}^{3} / \mathrm{s}\right)\end{array} \\ 7-12-60 & 3.36\end{array}$

Little Turkey Creek near Erick, Oklahoma, Miscellaneous site.

Lat. $35^{\circ} 17^{\prime} 30^{\prime \prime}$, long. $99^{\circ} 52^{\circ} 00^{\prime \prime}, \mathrm{SW}^{\prime} \mathrm{I}^{\prime} 4 \mathrm{sec} .32$, T.10 N., R.25 W., at bridge on county road $5 \mathrm{miles}$ north of Erick, Okla.

\begin{tabular}{|c|c|c|c|c|c|c|c|}
\hline Date & $\begin{array}{c}\text { Discharge } \\
\left(\mathrm{ft}^{3} / \mathrm{s}\right)\end{array}$ & Date & $\begin{array}{c}\text { Discharge } \\
\left(\mathrm{ft}^{3} / \mathrm{s}\right)\end{array}$ & Date & $\begin{array}{c}\text { Discharge } \\
\left(\mathrm{ft}^{3} / \mathrm{s}\right)\end{array}$ & Date & $\begin{array}{c}\text { Discharge } \\
\left(\mathrm{ft}^{3} / \mathrm{s}\right)\end{array}$ \\
\hline $\begin{array}{r}12-9-52 \\
11-6-53 \\
3-9-54 \\
4-20-54\end{array}$ & $\begin{array}{l}.84 \\
.69 \\
.69 \\
.50\end{array}$ & $\begin{array}{r}7-2-54 \\
8-30-54 \\
11-16-54\end{array}$ & $\begin{array}{l}.70 \\
.24 \\
.16\end{array}$ & $\begin{array}{r}12-16-54 \\
2-28-55 \\
3-30-55\end{array}$ & $\begin{array}{l}.15 \\
.23 \\
.18\end{array}$ & $\begin{array}{l}4-28-55 \\
7-12-55 \\
8-30-55\end{array}$ & $\begin{array}{l}.13 \\
.11 \\
.07\end{array}$ \\
\hline
\end{tabular}

Little Washita near Ninnekah, Oklahoma, Miscellaneous site.

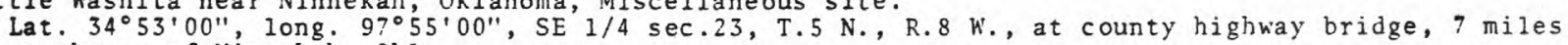
southwest of Ninnekah, Okla.

\begin{tabular}{|c|c|c|c|c|c|c|c|}
\hline Date & $\begin{array}{c}\text { Discharge } \\
\left(\mathrm{ft}^{3} / \mathrm{s}\right)\end{array}$ & Date & $\begin{array}{c}\text { Discharge } \\
\left(\mathrm{ft}^{3} / \mathrm{s}\right)\end{array}$ & Date & $\begin{array}{c}\text { Discharge } \\
\left(\mathrm{ft}^{3} / \mathrm{s}\right)\end{array}$ & Date & $\begin{array}{c}\text { Discharge } \\
\left(\mathrm{ft}^{3} / \mathrm{s}\right)\end{array}$ \\
\hline $\begin{array}{l}3-22-49 \\
4-5-49\end{array}$ & $\begin{array}{l}39.4 \\
30.4\end{array}$ & $4-13-49$ & 31.9 & $4-29-49$ & 34.0 & $12-30-48$ & 10.0 \\
\hline
\end{tabular}

Lost Creek at Seneca, Missouri, 07188500 , partial record site.

Lat. $36^{\circ} 50^{\prime} 28^{\prime \prime}$, long. $94^{\circ} 36^{\prime} 30^{\prime \prime}$, in SE $1 / 4$ SE $1 / 4 \mathrm{sec} .35, \mathrm{~T} .25 \mathrm{~N} ., \mathrm{R} .34 \mathrm{~W}^{\prime}$, Newton County, at Seneca Street bridge in Seneca, 0.5 mile upstream from Little Lost Creek and 9.5 miles upstream from mouth.

\begin{tabular}{|c|c|c|c|c|c|c|c|}
\hline Date & $\begin{array}{c}\text { Discharge } \\
\left(\mathrm{ft}^{3} / \mathrm{s}\right)\end{array}$ & Date & $\begin{array}{c}\text { Discharge } \\
\left(\mathrm{ft}^{3} / \mathrm{s}\right)\end{array}$ & Date & $\begin{array}{c}\text { Discharge } \\
\left(\mathrm{ft}^{3} / \mathrm{s}\right)\end{array}$ & Date & $\begin{array}{c}\text { Discharge } \\
\left(\mathrm{ft}^{3} / \mathrm{s}\right)\end{array}$ \\
\hline $\begin{array}{r}12-27-60 \\
2-13-61 \\
3-7-61 \\
4-19-61 \\
7-11-61 \\
8-3-61 \\
11-9-61 \\
2-14-62 \\
3-26-62 \\
6-25-62\end{array}$ & $\begin{array}{l}11.6 \\
6.55 \\
54.2 \\
26.9 \\
15.4 \\
29.3 \\
37.7 \\
21.9 \\
55.2 \\
20.7\end{array}$ & $\begin{array}{r}10-8-62 \\
12-27-62 \\
4-8-63 \\
10-14-63 \\
10-22-64 \\
2-4-65 \\
6-7-65 \\
9-15-65 \\
2-24-66 \\
1-18-67\end{array}$ & $\begin{array}{c}40.8 \\
27.7 \\
14.0 \\
2.54 \\
4.27 \\
7.58 \\
12.6 \\
9.0 \\
19.9 \\
7.38\end{array}$ & $\begin{array}{r}5-17-67 \\
9-29-67 \\
11-17-67 \\
5-21-68 \\
9-17-68 \\
10-21-68 \\
1-8-69 \\
5-14-69 \\
9-11-69 \\
1-28-70\end{array}$ & $\begin{array}{r}21.8 \\
5.51 \\
21.0 \\
21.8 \\
17.2 \\
8.2 \\
43.0 \\
26.0 \\
8.0 \\
17.0\end{array}$ & $\begin{array}{r}4-15-70 \\
8-18-70 \\
11-30-70 \\
1-21-71 \\
6-23-71 \\
8-17-71 \\
11-16-71 \\
2-9-72 \\
5-23-72 \\
8-9-72\end{array}$ & $\begin{array}{r}28.0 \\
7.2 \\
26.0 \\
24.0 \\
9.6 \\
6.2 \\
5.1 \\
8.9 \\
12.0 \\
2.8\end{array}$ \\
\hline
\end{tabular}


Lost Creek near Washita, Oklahoma, Miscellaneous site.

Lat. $35^{\circ} 06^{\prime} 55^{\prime \prime}$, Iong. $98^{\circ} 18^{\prime} 43^{\prime \prime}$, on west line of sec.1, T.7 N., R.11 W., at county highway bridge, $1-1 / 2$ miles northeast of Washita, Okla.

$\begin{array}{cr}\text { Date } & \begin{array}{r}\text { Discharge } \\ \left(\mathrm{ft}^{3} / \mathrm{s}\right)\end{array} \\ 8-30-56 & .067\end{array}$

Main Creek near Waynoka, Oklahoma, 07158010 , partial record site.

Lat. $36^{\circ} 29^{\prime} 30^{\prime \prime}$, long. $98^{\circ} 53^{\prime} 30^{\prime \prime}$, on south line SE $1 / 4$ SE 1/4 sec.3, T.23 N., R.16 W., Major County, at county road' bridge on FAS road, $0.5 \mathrm{mile}$ west of U.S. Highway 281 , $6 \mathrm{miles}$ south of Waynoka and about 2 miles upstream from mouth.

\begin{tabular}{|c|c|c|c|c|c|c|c|}
\hline Date & $\begin{array}{c}\text { Discharge } \\
\left(f t^{3} / \mathrm{s}\right)\end{array}$ & Date & $\begin{array}{c}\text { Discharge } \\
\left(\mathrm{ft}^{3} / \mathrm{s}\right)\end{array}$ & Date & $\begin{array}{c}\text { Discharge } \\
\left(\mathrm{ft}^{3} / \mathrm{s}\right)\end{array}$ & Date & $\begin{array}{c}\text { Discharge } \\
\left(f t^{3} / \mathrm{s}\right)\end{array}$ \\
\hline $\begin{array}{r}10-28-64 \\
3-4-65 \\
4-28-65 \\
7-21-65 \\
10-6-65 \\
2-16-66 \\
4-13-66 \\
7-8-66 \\
11-22-66\end{array}$ & $\begin{array}{l}3.92 \\
2.48 \\
6.03 \\
0 \\
8.7 \\
12.0 \\
7.0 \\
0 \\
0\end{array}$ & $\begin{array}{r}2-2-67 \\
4-27-67 \\
7-27-67 \\
10-19-67 \\
2-8-68 \\
4-18-68 \\
7-25-68 \\
11-21-68 \\
3-13-69\end{array}$ & $\begin{array}{l}5.4 \\
6.0 \\
2.6 \\
2.2 \\
5.0 \\
1.9 \\
0 \\
6.4 \\
8.6\end{array}$ & $\begin{array}{r}6-19-69 \\
7-30-69 \\
12-12-69 \\
3-3-70 \\
4-8-70 \\
8-13-70 \\
11-18-70 \\
3-11-71 \\
6-8-71\end{array}$ & $\begin{array}{l}8.6 \\
.59 \\
6.2 \\
6.0 \\
8.5 \\
2.1 \\
5.7 \\
8.8 \\
1.7\end{array}$ & $\begin{array}{r}9-16-71 \\
9-29-71 \\
12-8-71 \\
2-18-72 \\
5-24-72 \\
8-4-72 \\
10-26-72 \\
3-14-73 \\
5-18-73\end{array}$ & $\begin{array}{l}0 \\
3.1 \\
8.4 \\
6.0 \\
3.7 \\
.29 \\
4.30 \\
7.30 \\
16.0\end{array}$ \\
\hline
\end{tabular}

Main Spring near Woodward, Oklahoma, Miscellaneous site.

Lat. $36^{\circ} 25^{\prime} 09^{\prime \prime}$, long. $99^{\circ} 15^{\prime} 25^{\prime \prime}$, SE $1 / 4$ sec.23, T. 23 N., R. 20 W., at Boiling Springs State Park, 5 miles east of Woodward, okla.
Date
Discharge
$\left(\mathrm{ft}^{3} / \mathrm{s}\right)$
$6-6-56$
.04

Mill Creek near Mill Creek, Oklahoma, 07331200 , partial record site.

Lat. $34^{\circ} 24^{\prime} 18^{\prime \prime}$, long. $96^{\circ} 51^{\prime} 47^{\prime \prime}$, in NW $1 / 4 \mathrm{~s}$ ec.11, T.2 S., R.4 E., Johnston County, at county road bridge, 2 miles west of Mill Creek, Okla.

\begin{tabular}{|c|c|c|c|c|c|c|c|}
\hline Date & $\begin{array}{c}\text { Discharge } \\
\left(\mathrm{ft}^{3} / \mathrm{s}\right)\end{array}$ & Date & $\begin{array}{c}\text { Discharge } \\
\left(\mathrm{ft}^{3} / \mathrm{s}\right)\end{array}$ & Date & $\begin{array}{c}\text { Discharge } \\
\left(\mathrm{ft}^{3} / \mathrm{s}\right)\end{array}$ & Date & $\begin{array}{c}\text { Discharge } \\
\left(\mathrm{ft}^{3} / \mathrm{s}\right)\end{array}$ \\
\hline $\begin{array}{r}9-3-51 \\
10-9-52 \\
11-18-52 \\
12-23-52 \\
2-4-53 \\
3-10-53 \\
4-14-53 \\
6-2-53 \\
6-23-53 \\
8-19-53 \\
9-30-53 \\
11-17-53 \\
1-12-54 \\
2-25-54 \\
4-20-54 \\
5-28-54 \\
7-14-54 \\
8-24-54 \\
9-16-54\end{array}$ & $\begin{array}{l}2.15 \\
1.22 \\
2.01 \\
2.06 \\
1.28 \\
2.92 \\
5.19 \\
7.05 \\
3.66 \\
6.28 \\
2.01 \\
3.74 \\
2.67 \\
3.51 \\
1.68 \\
14.3 \\
4.82 \\
3.03 \\
1.25\end{array}$ & $\begin{array}{r}11-23-54 \\
12-21-54 \\
2-1-55 \\
3-1-55 \\
6-13-55 \\
7-27-55 \\
9-21-55 \\
1-17-58 \\
3-19-58 \\
6-11-58 \\
9-5-58 \\
11-5-58 \\
1-8-59 \\
6-9-59 \\
9-15-59 \\
10-27-59 \\
11-23-59 \\
12-8-59 \\
1-27-60\end{array}$ & $\begin{array}{c}1.96 \\
2.00 \\
2.55 \\
3.07 \\
1.68 \\
1.42 \\
.95 \\
7.84 \\
19.4 \\
7.01 \\
4.81 \\
2.54 \\
2.73 \\
2.32 \\
3.72 \\
5.11 \\
9.85 \\
3.60 \\
16.5\end{array}$ & $\begin{array}{r}5-3-60 \\
8-25-60 \\
12-1-60 \\
1-12-61 \\
3-1-61 \\
4-5-61 \\
5-17-61 \\
11-30-61 \\
8-22-62 \\
7-22-63 \\
10-11-63 \\
3-10-65 \\
6-9-65 \\
7-14-65 \\
11-30-65 \\
3-23-66 \\
6-13-66 \\
11-22-66 \\
2-15-67\end{array}$ & $\begin{array}{c}12.0 \\
3.91 \\
5.31 \\
11.2 \\
11.0 \\
18.6 \\
5.47 \\
10.8 \\
2.94 \\
3.44 \\
1.04 \\
6.44 \\
7.90 \\
3.47 \\
1.4 \\
1.5 \\
2.0 \\
2.6 \\
1.4\end{array}$ & $\begin{array}{r}6-7-67 \\
8-8-67 \\
12-11-67 \\
2-20-68 \\
4-24-68 \\
9-19-68 \\
12-17-68 \\
3-12-69 \\
6-5-69 \\
9-17-69 \\
12-12-69 \\
2-19-70 \\
5-12-70 \\
8-4-70 \\
11-19-70 \\
3-10-71 \\
6-9-71 \\
7-8-71\end{array}$ & $\begin{array}{r}8.8 \\
5.1 \\
5.8 \\
17.0 \\
17.0 \\
4.3 \\
11.0 \\
24.0 \\
19.0 \\
3.2 \\
4.5 \\
4.3 \\
14.0 \\
4.2 \\
14.0 \\
4.5 \\
3.6 \\
2.6\end{array}$ \\
\hline
\end{tabular}


Mill Creek near Ravia, Oklahoma, Miscellaneous site.

Lat. $34^{\circ} 13^{\prime} 15^{\prime \prime}$, long. $96^{\circ} 49^{\prime} 00^{\prime \prime}$, SE $1 / 4$ sec.6, T.4 S., R.5 E., at county highway bridge $3.1 / 2 \mathrm{miles}$ southwest of Ravia, Oklahoma.

\begin{tabular}{|c|c|c|c|c|c|c|c|}
\hline Date & $\begin{array}{c}\text { Discharge } \\
\left(\mathrm{ft}^{3} / \mathrm{s}\right)\end{array}$ & Date & $\begin{array}{c}\text { Discharge } \\
\left(\mathrm{ft}^{3} / \mathrm{s}\right)\end{array}$ & Date & $\begin{array}{c}\text { Discharge } \\
\left(\mathrm{ft}^{3} / \mathrm{s}\right)\end{array}$ & Date & $\begin{array}{c}\text { Discharge } \\
\left(\mathrm{ft}^{3} / \mathrm{s}\right)\end{array}$ \\
\hline $\begin{array}{l}7-19-49 \\
8-30-49\end{array}$ & $\begin{array}{l}5.36 \\
1.49\end{array}$ & $6-30-50$ & 10.80 & $11 \cdot 1 \cdot 50$ & 4.80 & $3 \cdot 1 \cdot 55$ & 20.8 \\
\hline
\end{tabular}

Montezuma Creek near Shulter, Oklahoma, Miscellaneous site.

Lat. $35^{\circ} 32^{\prime} 15^{\prime \prime}$, long. $95^{\circ} 57^{\prime} 30^{\prime \prime}$, on south line of sec.5, T.12 N., R.13 E., at bridge on U.S. Highway 75,1 mile north of Schulter, Okla.

\begin{tabular}{|c|c|c|c|c|c|c|c|}
\hline Date & $\begin{array}{c}\text { Discharge } \\
\left(\mathrm{ft}^{3} / \mathrm{s}\right)\end{array}$ & Date & $\begin{array}{c}\text { Discharge } \\
\left(\mathrm{ft}^{3} / \mathrm{s}\right)\end{array}$ & Date & $\begin{array}{c}\text { Discharge } \\
\left(\mathrm{ft}^{3} / \mathrm{s}\right)\end{array}$ & Date & $\begin{array}{c}\text { Discharge } \\
\left(\mathrm{ft}^{3} / \mathrm{s}\right)\end{array}$ \\
\hline $\begin{array}{l}3-15-56 \\
4-10-56 \\
5-8-56\end{array}$ & $\begin{array}{l}0 \\
0 \\
0\end{array}$ & $\begin{array}{l}5-30-56 \\
7-8-56 \\
8-14-56\end{array}$ & $\begin{array}{l}0^{.47} \\
0\end{array}$ & $\begin{array}{r}11-14-56 \\
1-3-57 \\
2-6-57\end{array}$ & $\begin{array}{l}n \\
0 \\
.40\end{array}$ & $\begin{array}{l}3-25-57 \\
7-9-57 \\
9-5-57\end{array}$ & $\begin{array}{l}1.77 \\
.20 \\
0\end{array}$ \\
\hline
\end{tabular}

Mountain Fork River near Eagleton, Oklahoma, Miscellaneous site.

Lat. $34^{\circ} 08^{\prime} 30^{\prime \prime}$, long. $94^{\circ} 42^{\prime} 30^{\prime \prime}$, NW $1 / 4$ sec.8, T.5 S., R.25 E., at Beaver Bend State Park, $9-1 / 2 \mathrm{miles}$ northwest of Eagleton, Okla.

\begin{tabular}{|c|c|c|c|c|c|c|c|}
\hline Date & $\begin{array}{c}\text { Discharge } \\
\left(\mathrm{ft}^{3} / \mathrm{s}\right)\end{array}$ & Date & $\begin{array}{c}\text { Discharge } \\
\left(\mathrm{ft}^{3} / \mathrm{s}\right)\end{array}$ & Date & $\begin{array}{c}\text { Discharge } \\
\left(\mathrm{ft}^{3} / \mathrm{s}\right)\end{array}$ & Date & $\begin{array}{c}\text { Discharge } \\
\left(\mathrm{ft}^{3} / \mathrm{s}\right)\end{array}$ \\
\hline $\begin{array}{l}7-22-54 \\
8-3-54\end{array}$ & $\begin{array}{l}.64 \\
.07\end{array}$ & $\begin{array}{l}7-22-54 \\
8-\quad 3-54\end{array}$ & $\begin{array}{r}3.53 \\
.22\end{array}$ & $\begin{array}{l}7-22-54 \\
8-\quad 3-54\end{array}$ & $\begin{array}{l}5.26 \\
2.87\end{array}$ & $7-10-60$ & 0 \\
\hline
\end{tabular}

Mud Creek near Grady, Oklahoma, Miscellaneous site.

Lat. $34^{\circ} 00^{\prime} 00^{\prime \prime}$, long. $35^{\prime} 00^{\prime \prime}$, SE $1 / 4 \mathrm{sec} .25$, T.6 S., R. 4 W., at bridge on State Highway 89 , 6 miles east of Grady, Okla.

$\begin{array}{rrrrrrrc}\text { Date } & \begin{array}{c}\text { Discharge } \\ \left(\mathrm{ft}^{3} / \mathrm{s}\right)\end{array} & \text { Date } & \begin{array}{c}\text { Discharge } \\ \left(\mathrm{ft}^{3} / \mathrm{s}\right)\end{array} & \text { Date } & \begin{array}{c}\text { Discharge } \\ \left(\mathrm{ft}^{3} / \mathrm{s}\right)\end{array} & \begin{array}{c}\text { Date } \\ \text { Discharge } \\ \left(\mathrm{ft}^{3} / \mathrm{s}^{2}\right)\end{array} \\ 9-6-51 & .44 & 7-23-52 & 0 & 12-22-52 & 0 & 6-1-53 & 0 \\ 11-26-51 & .27 & 9-3-52 & 0 & 2-3-53 & 0 & 6-22-53 & 0 \\ 1-15-52 & .28 & 10-9-52 & 0 & 3-9-53 & 0 & 8-18-53 & 5.66 \\ 3-4-52 & 9.20 & 11-17-52 & 0 & 4-13-53 & 1.28 & 9-23-53 & 0 \\ 6-12-52 & 2.64 & & & & & \end{array}$

Muddy Boggy Creek near Parker, Oklahoma, 07332700 , partial record site.

Lat. $34^{\circ} 44^{\prime} 28^{\prime \prime}$, long. $96^{\circ} 15^{\prime} 51^{\prime \prime}$, in SW $1 / 4$ sec.9, T.3 N., R.10 E., Coal County, at bridge on U.S. Highway 75,5 miles west of Parker.

\begin{tabular}{|c|c|c|c|c|c|c|c|}
\hline Date & $\begin{array}{c}\text { Discharge } \\
\left(\mathrm{ft}^{3} / \mathrm{s}\right)\end{array}$ & Date & $\begin{array}{c}\text { Discharge } \\
\left(\mathrm{ft}^{3} / \mathrm{s}\right)\end{array}$ & Date & $\begin{array}{c}\text { Discharge } \\
\left(\mathrm{ft}^{3} / \mathrm{s}\right)\end{array}$ & Date & $\begin{array}{c}\text { Discharge } \\
\left(\mathrm{ft}^{3} / \mathrm{s}\right)\end{array}$ \\
\hline $\begin{array}{r}12-12-57 \\
2-24-58 \\
4-7-58 \\
8-27-59 \\
9-14-59 \\
10-26-59 \\
12-7-59 \\
3-21-60 \\
9-13-60 \\
11-29-60 \\
1-10-61 \\
2-27-61 \\
3-21-61 \\
4-4-61 \\
5-15-61\end{array}$ & $\begin{array}{r}12.4 \\
22.8 \\
32.3 \\
1.7 \\
.9 \\
17.6 \\
16.0 \\
32.9 \\
.7 \\
11.5 \\
20.8 \\
18.3 \\
20.9 \\
22.4 \\
12.9\end{array}$ & $\begin{array}{r}10-31-61 \\
2-12-62 \\
5-18-62 \\
7-11-62 \\
8-15-62 \\
9-20-62 \\
2-7-63 \\
4-23-63 \\
8-20-63 \\
10-11-63 \\
12-3-63 \\
2-18-64 \\
4-11-64 \\
12-14-64 \\
3-9-65\end{array}$ & $\begin{array}{c}12.2 \\
18.5 \\
8.68 \\
.15 \\
0 \\
5.68 \\
17.2 \\
10.9 \\
0 \\
0 \\
0 \\
3.03 \\
7.72 \\
11.3 \\
6.21\end{array}$ & $\begin{array}{r}5-24-65 \\
12-1-65 \\
3-17-66 \\
6-9-66 \\
8-30-66 \\
11-22-66 \\
2-14-67 \\
5-25-67 \\
8-29-68 \\
12-5-68 \\
2-14-68 \\
5-7-68 \\
9-10-68 \\
12-11-68 \\
6-19-69\end{array}$ & $\begin{array}{c}4.34 \\
.07 \\
4.0 \\
.54 \\
.22 \\
.08 \\
.38 \\
7.3 \\
.19 \\
3.5 \\
22.0 \\
11.0 \\
2.6 \\
19.0 \\
38.0\end{array}$ & $\begin{array}{r}7-8-69 \\
12-17-69 \\
2-18-70 \\
4-9-70 \\
8-11-70 \\
11-30-70 \\
3-10-71 \\
6-23-71 \\
8-31-71 \\
3-8-71 \\
6-22-72 \\
8-2-72 \\
12-5-72 \\
2-21-73 \\
5-15-73\end{array}$ & $\begin{array}{c}1.8 \\
8.3 \\
11.0 \\
22.0 \\
0 \\
15.0 \\
18.0 \\
13.0 \\
.63 \\
11.0 \\
. .78 \\
0 \\
25 . \\
23 . \\
45 .\end{array}$ \\
\hline
\end{tabular}


Mulberry Creek near Mangum, Oklahoma, Miscellaneous site.

Lat. $34^{\circ} 31^{\prime} 28^{\prime \prime}$, long. $98^{\circ} 38^{\circ} 00^{\prime \prime}$, SW $1 / 4$ sec.4, T.4 N., R. $23 \mathrm{~W}$., at bridge on county road $7.1 / 2 \mathrm{miles}$ southwest of Mangum, okla.

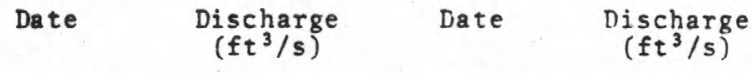

$\begin{array}{rlll}2-16-52 & .76 & 4-22-54 & .98\end{array}$

McGee Creek near Farris, Oklahoma, Miscellaneous site.

Lat. $34^{\circ} 18^{\prime} 45^{\prime \prime}$, long. $95^{\circ} 52^{\prime} 30^{\prime \prime}, \mathrm{NW} 1 / 4 \mathrm{sec} .7, \mathrm{~T} .3 \mathrm{~S} ., \mathrm{R} .14 \mathrm{E}$. , at site $3-1 / 2 \mathrm{miles}$ north of Farris, Ok1a.

\begin{tabular}{|c|c|c|c|c|c|c|c|}
\hline Date & $\begin{array}{c}\text { Discharge } \\
\left(\mathrm{ft}^{3} / \mathrm{s}\right)\end{array}$ & Date & $\begin{array}{c}\text { Discharge } \\
\left(\mathrm{ft}^{3} / \mathrm{s}\right)\end{array}$ & Date & $\begin{array}{c}\text { Discharge } \\
\left(\mathrm{ft}^{3} / \mathrm{s}\right)\end{array}$ & Date & $\begin{array}{c}\text { Discharge } \\
\left(\mathrm{ft}^{3} / \mathrm{s}\right)\end{array}$ \\
\hline $\begin{array}{l}3-1-56 \\
2-25-58\end{array}$ & $\begin{array}{l}11.7 \\
17.6\end{array}$ & $\begin{array}{r}4-9-58 \\
11-4-58\end{array}$ & $\begin{array}{l}48.8 \\
.24\end{array}$ & $\begin{array}{l}1-29-59 \\
8-26-59\end{array}$ & $\begin{array}{r}1.65 \\
.40\end{array}$ & $9-14-59$ & .17 \\
\hline
\end{tabular}

Noble Springs near Texola, Oklahoma, Miscellaneous site.

Lat. $34^{\circ} 47^{\prime} 46^{\prime \prime}$, long. $99^{\circ} 32^{\prime} 34^{\prime \prime}$, SW $1 / 4 \mathrm{sec} .28$, T. 4 N., R. 22 W., 6 miles north of Texola, Okla.

Date Discharge

North Boggy Creek near Atoka, Oklahoma, Miscellaneous site.

Lat. $34^{\circ} 31^{\prime} 00^{\prime \prime}$, long. $96^{\circ} 05^{\prime} 30^{\prime \prime}, \mathrm{SW} 1 / 4 \mathrm{sec} 7, \mathrm{~T} .1 \mathrm{~S} ., \mathrm{R} .12 \mathrm{E} ., 6$ miles northeast of A.toka, Okla.

\begin{tabular}{|c|c|c|c|c|c|c|c|}
\hline Date & $\begin{array}{c}\text { Discharge } \\
\left(\mathrm{ft}^{3} / \mathrm{s}\right)\end{array}$ & Date & $\begin{array}{c}\text { Discharge } \\
\left(\mathrm{ft}^{3} / \mathrm{s}\right)\end{array}$ & Date & $\begin{array}{c}\text { Discharge } \\
\left(\mathrm{ft}^{3} / \mathrm{s}\right)\end{array}$ & Date & $\begin{array}{c}\text { Discharge } \\
\left(\mathrm{ft}^{3} / \mathrm{s}\right)\end{array}$ \\
\hline $\begin{array}{l}7-20-49 \\
8-30-49\end{array}$ & $\begin{array}{l}.48 \\
.08\end{array}$ & $\begin{array}{r}11-1-50 \\
4-8-58\end{array}$ & $\begin{array}{l}58.90 \\
42.3\end{array}$ & $\begin{array}{l}6-10-58 \\
7-16-58\end{array}$ & $\begin{array}{l}2.58 \\
21.2\end{array}$ & $\begin{array}{r}11-4-58 \\
1-29-59\end{array}$ & $\begin{array}{l}.60 \\
.36\end{array}$ \\
\hline
\end{tabular}

North Fork Red River near Carter, Oklahoma, Miscellaneous site.

Lat. $35^{\circ} 10^{\prime} 05^{\prime \prime}$, long. $99^{\circ} 30^{\prime} 25^{\prime \prime}$, in NW $1 / 4 \mathrm{SE} 1 / 4 \mathrm{sec} .15, \mathrm{~T} .8 \mathrm{~N}$, , R.22 W., Beckham County, near left

bank on downstream side of pier of bridge on State Highway $34,3.0 \mathrm{mi}$ south of Carter, $10.8 \mathrm{mi}$ downstream from Tinker Creek and at mile 110.5 .

$\begin{array}{lccccccc}\text { Date } & \begin{array}{c}\text { Discharge } \\ \left(\mathrm{ft}^{3} / \mathrm{s}\right)\end{array} & \text { Date } & \begin{array}{c}\text { Discharge } \\ \left(\mathrm{ft}^{3} / \mathrm{s}\right)\end{array} & \text { Date } & \begin{array}{c}\text { Discharge } \\ \left(\mathrm{ft}^{3} / \mathrm{s}\right)\end{array} & \begin{array}{c}\text { Date } \\ \text { Discharge } \\ \left(\mathrm{ft}^{3} / \mathrm{s}\right)\end{array} \\ 3-8-63 & 104.5 & 8-5-63 & 0 & 10-10-63 & 0 & 10-20-63 & 0 \\ 5-6-63 & 11.3 & & & & 0-30-64 & 4.33\end{array}$

North Fork Red River near Frederick, Oklahoma, Miscellaneous site.

Lat. $34^{\circ} 20^{\prime} 32^{\prime \prime}$, long. $99^{\circ} 11^{\prime} 15^{\prime \prime}$, NW $1 / 4 \mathrm{sec} .33$, T.2 S., R.19 W., half a mile upstream from mouth and 11 miles southwest of Frederick, Okla.

$\begin{array}{lccc}\text { Date } & \begin{array}{c}\text { Discharge } \\ \left(\mathrm{ft}^{3} / \mathrm{s}\right)\end{array} & \text { Date } & \begin{array}{c}\text { Discharge } \\ \left(\mathrm{ft}^{3} / \mathrm{s}\right)\end{array} \\ 2-13-53 & 0 & 4-22-53 & 15.2\end{array}$

North Fork of Red River near Granite, Oklahoma, Miscellaneous site.

Lat. $35^{\circ} 00^{\prime} 00^{\prime \prime}$, long. $99^{\circ} 21^{\prime} 43^{\prime \prime}$, NE $1 / 4 \mathrm{sec} .19$, T.6 N., R. $20 \mathrm{~W} ., 1 \mathrm{mile}$ above Lake Altus and 2 miles northeast of Granite, Okla.

$\begin{array}{cccccc}\text { Date } & \begin{array}{c}\text { Discharge } \\ \left(\mathrm{ft}^{3} / \mathrm{s}\right)\end{array} & \text { Date } & \begin{array}{c}\text { Discharge } \\ \left(\mathrm{ft}^{3} / \mathrm{s}\right)\end{array} & \text { Date } & \begin{array}{c}\text { Discharge } \\ \left(\mathrm{ft}^{3} / \mathrm{s}\right)\end{array} \\ 10-30-50 & 33.0 & 11-15-50 & 46.0 & 12-11-51 & 30.2\end{array}$


North Fork Red River near Humphreys, Oklahoma, Miscelianeous site.

Lat. $34^{\circ} 31^{\prime} 37^{\prime \prime}$, long. $99^{\circ} 14^{\circ} 00^{\prime \prime}, \mathrm{NE} 1 / 4 \mathrm{NE} 1 / 4 \mathrm{sec} .28, \mathrm{~T} .1 \mathrm{~N}, \mathrm{~N}^{\prime}$.19 W., at site 3 miles southeast of Humphreys, Okla.

$\begin{array}{crrr}\text { Date. } & \begin{array}{r}\text { Discharge } \\ \left(\mathrm{ft}^{3} / \mathrm{s}\right)\end{array} & \text { Date } & \begin{array}{r}\text { Discharge } \\ \left(\mathrm{ft}^{3} / \mathrm{s}\right)\end{array} \\ 2-13-53 & 1.69 & 4-22-53 & 11.70\end{array}$

North Fork Red River near Mayfield, Oklahoma, Miscellaneous site.

Lat. $35^{\circ} 18^{\prime} 14^{\prime \prime}$, long. $99^{\circ} 57^{\prime} 08^{\prime \prime}$, NW $1 / 4 \mathrm{sec} .22$, T.10 N., R. $26 \mathrm{~W}$., at county highway bridge, $3-1 / 2$ miles west of Mayfield, Okla.

$\begin{array}{cc}\text { Date } & \begin{array}{c}\text { Discharge } \\ \left(\mathrm{ft}^{3} / \mathrm{s}\right)\end{array} \\ 4-18-51 & 22.4\end{array}$

North Fork Red River near Texola, Oklahoma, Miscellaneous site.

Lat. $35^{\circ} 17^{\prime} 00^{\prime \prime}$, long. $99^{\circ} 59^{\circ} 00^{\prime \prime}$, SW $1 / 4 \mathrm{sec} 31$, T.10 N., R. $26 \mathrm{~W}$, at county highway bridge, $5 \mathrm{miles}$ north of Texola, Okla.

\begin{tabular}{|c|c|c|c|c|c|c|c|}
\hline Date & $\begin{array}{c}\text { Discharge } \\
\left(\mathrm{ft}^{3} / \mathrm{s}\right)\end{array}$ & Date & $\begin{array}{c}\text { Discharge } \\
\left(\mathrm{ft}^{3} / \mathrm{s}\right)\end{array}$ & Date & $\begin{array}{c}\text { Discharge } \\
\left(\mathrm{ft}^{3} / \mathrm{s}\right)\end{array}$ & Date & $\begin{array}{c}\text { Discharge } \\
\left(\mathrm{ft}^{3} / \mathrm{s}\right)\end{array}$ \\
\hline $\begin{array}{r}4-18-51 \\
12-11-51 \\
2-6-52\end{array}$ & $\begin{array}{r}.47 \\
5.50 \\
7.21\end{array}$ & $\begin{array}{r}3-24-52 \\
8-20-52 \\
10-28-52\end{array}$ & $\begin{array}{l}0 \\
0 \\
0\end{array}$ & $\begin{array}{l}1-6-53 \\
3-11-53 \\
9-29-53\end{array}$ & $\begin{array}{l}0 \\
0 \\
0\end{array}$ & $\begin{array}{l}11-16-53 \\
12-23-53\end{array}$ & $0^{.05}$ \\
\hline
\end{tabular}

North Fork Red River near Tipton, Oklahoma, Miscellaneous site.

Lat. $34^{\circ} 32^{\prime} 00^{\prime \prime}$, long. $99^{\circ} 12^{\circ} 0^{\prime \prime}$, NE $1 / 4$ sec.28, T.1 N., R.19 w., 2 miles below mouth of otter Creek and 4 miles northwest of Tipton, Oklahoma

\begin{tabular}{|c|c|c|c|c|c|c|c|}
\hline Date & $\begin{array}{c}\text { Discharge } \\
\left(\mathrm{ft}^{3} / \mathrm{s}\right)\end{array}$ & Date & $\begin{array}{c}\text { Discharge } \\
\left(\mathrm{ft}^{3} / \mathrm{s}\right)\end{array}$ & Date & $\begin{array}{c}\text { Discharge } \\
\left(\mathrm{ft}^{3} / \mathrm{s}\right)\end{array}$ & Date & $\begin{array}{c}\text { Discharge } \\
\left(\mathrm{ft}^{3} / \mathrm{s}\right)\end{array}$ \\
\hline $\begin{array}{l}11-29-45 \\
11-29-45\end{array}$ & $\begin{array}{l}15.5 \\
14.4\end{array}$ & $11-30-45$ & 52.6 & $2-13-53$ & .89 & $4-22-53$ & 12.8 \\
\hline
\end{tabular}

North Fork Red Riyer near Nillow, Oklahoma, Miscellaneous site.

Lat. $35^{\circ} 03^{\prime} 00^{\circ}$, long. $99^{\circ} 22^{\prime} 51^{\prime \prime}$, NE $1 / 4 \mathrm{sec} .24$, T.7 N., R. $21 \mathrm{w}$, at county highway bridge, $8 \mathrm{miles}$ east of Willow, Okla.

$\begin{array}{cccccc}\text { Date } & \begin{array}{c}\text { Discharge } \\ \left(\mathrm{ft}^{3} / \mathrm{s}\right)\end{array} & \text { Date } & \begin{array}{c}\text { Discharge } \\ \left(\mathrm{ft}^{3} / \mathrm{s}\right)\end{array} & \text { Date } & \begin{array}{c}\text { Discharge } \\ \left(\mathrm{ft}^{3} / \mathrm{s}\right)\end{array} \\ 10-30-50 & 30.0 & 11-15-50 & 48.9 & 12-11-51 & 24.8\end{array}$

Oil Creek near Baum, Oklahoma, Miscellaneous site.

Lat. $34^{\circ} 15^{\prime} 14^{\prime \prime}$, long. $96^{\circ} 55^{\prime} 43^{\prime \prime}$, NE $1 / 4$ sec. 30 , T.3 S., R.4 E., 2-1/2 miles northeast of Baum, Ok1a.

$\begin{array}{crrr}\text { Date } & \begin{array}{c}\text { Discharge } \\ \left(\mathrm{ft}^{3} / \mathrm{s}\right)\end{array} & \text { Date } & \begin{array}{r}\text { Discharge } \\ \left(\mathrm{ft}^{3} / \mathrm{s}\right)\end{array} \\ 8-10-58 & 1.58 & 11-15-55 & 1.57\end{array}$


Omaha Creek near Ponca City, nklahoma, Miscellaneous site.

Lat. $36^{\circ} 39^{\prime} 28^{\prime \prime}$, long. $97^{\circ} 06^{\prime} 34^{\prime \prime}$, NW $1 / 4$ sec.16, T. 25 N., R. 2 E., at county highway bridge, $1-1 / 4$ miles west of White Eagle station, Okla.

Date

$10-22-52$

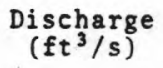

3.39
Date

$12-3 \cdot 52$

\section{Discharge
$\left(\mathrm{ft}^{3} / \mathrm{s}\right)$}

3.97

Palo Duro Creek near Range, Oklahoma, 07233700 , partial record site.

Lat. $36^{\circ} 37^{\prime} 00^{\prime \prime}$, 1ong. $101^{\circ} 01^{\prime} 24^{\prime \prime}$, in SW $1 / 4 \mathrm{sec} .21, \mathrm{~T} .2 \mathrm{~N} ., \mathrm{R} .19 \mathrm{E}$., Texas County, at bridge on State Highway 3, 6 miles northeast of Range.

\begin{tabular}{|c|c|c|c|c|c|c|c|}
\hline Date & $\begin{array}{c}\text { Discharge } \\
\left(\mathrm{ft}^{3} / \mathrm{s}\right)\end{array}$ & Date & $\begin{array}{c}\text { Discharge } \\
\left(\mathrm{ft}^{3} / \mathrm{s}\right)\end{array}$ & Date & $\begin{array}{c}\text { Discharge } \\
\left(\mathrm{ft}^{3} / \mathrm{s}\right)\end{array}$ & Date & $\begin{array}{c}\text { Discharge } \\
\left(\mathrm{ft}^{3} / \mathrm{s}\right)\end{array}$ \\
\hline $\begin{array}{r}12-21-51 \\
2-29-52 \\
5-6-52 \\
6-13-52 \\
7-24-52 \\
8-14-52 \\
11-10-53 \\
12-29-53 \\
2-26-54 \\
4-27-54 \\
10-25-54 \\
2-9-55 \\
4-4-55 \\
7-7-55 \\
12-12-55 \\
3-23-56 \\
4-30-56 \\
8-28-56 \\
12-5-56 \\
11-29-57 \\
5-15-57 \\
2-21-58\end{array}$ & $\begin{array}{l}2.42 \\
8.74 \\
6.43 \\
0 \\
1.16 \\
0 \\
6.14 \\
5.13 \\
4.52 \\
1.96 \\
.73 \\
7.33 \\
2.34 \\
.76 \\
2.71 \\
3.63 \\
1.76 \\
1.84 \\
2.29 \\
1.58 \\
8.37 \\
6.12\end{array}$ & $\begin{array}{r}5-14-58 \\
10-21-58 \\
12-19-58 \\
2-16-59 \\
4-1-59 \\
9-2-59 \\
9-30-59 \\
11-24-59 \\
1-6-60 \\
2-16-60 \\
4-18-60 \\
10-12-60 \\
1-17-61 \\
3-2-61 \\
4-20-61 \\
11-7-61 \\
2-13-62 \\
9-25-62 \\
1-22-63 \\
3-15-63 \\
7-26-63\end{array}$ & $\begin{array}{c}6.57 \\
2.15 \\
13.6 \\
7.99 \\
6.10 \\
0 \\
0 \\
4.78 \\
5.44 \\
12.4 \\
3.15 \\
13.4 \\
11.3 \\
5.77 \\
7.31 \\
6.64 \\
6.38 \\
3.30 \\
2.85 \\
6.36 \\
0\end{array}$ & $\begin{array}{r}10-17-63 \\
12-6-63 \\
2-13-64 \\
6-3-64 \\
7-21-64 \\
8-31-64 \\
12-28-64 \\
3-2-65 \\
4-13-65 \\
8-10-65 \\
10-8-65 \\
2-15-66 \\
4-12-66 \\
7-6-66 \\
10-11-66 \\
1-23-67 \\
5-12-67 \\
8-28-67 \\
11-27-67 \\
2-5-68 \\
6-24-68\end{array}$ & $\begin{array}{l}0 \\
2.07 \\
8.42 \\
3.12 \\
0 \\
0 \\
7.69 \\
9.11 \\
3.57 \\
6.77 \\
1.8 \\
5.1 \\
4.8 \\
0 \\
2.6 \\
5.4 \\
0 \\
.33 \\
1.2 \\
4.9 \\
5.3\end{array}$ & $\begin{array}{r}8-13-68 \\
12-24-68 \\
2-28-69 \\
6-23-69 \\
8-12-69 \\
12-24-69 \\
3-24-70 \\
4-14-70 \\
8-3-70 \\
12-2-70 \\
2-16-71 \\
6-25-71 \\
7-26-71 \\
9-30-71 \\
12-9-71 \\
3-11-72 \\
6-6-72 \\
8-11-72 \\
12-18-72 \\
3-8-73 \\
5-30-73\end{array}$ & $\begin{array}{c}0 \\
7.0 \\
6.8 \\
3.1 \\
0 \\
7.2 \\
4.2 \\
3.5 \\
.64 \\
4.4 \\
5.2 \\
.68 \\
3.2 \\
1.6 \\
13.0 \\
5.2 \\
2.6 \\
0 \\
3.4 \\
6.9 \\
12.0\end{array}$ \\
\hline
\end{tabular}

Pennington Creek near Reagan, Oklahoma, 07331300 ,partial record site.

Lat. $34^{\circ} 21^{\prime} 51^{\prime \prime}$, long. $96^{\circ} 43^{\prime} 01^{\prime \prime}$, in SE $1 / 4$ sec. 30 , T.2 S., R. 6 E., Johnston County, at 1 ow-water dam, $0.8 \mathrm{mile}$ above bridge on State Highway $7,0.8$ mile northeast of Reagan, Okla.

\begin{tabular}{|c|c|c|c|c|c|c|c|}
\hline Date & $\begin{array}{c}\text { Discharge } \\
\left(\mathrm{ft}^{3} / \mathrm{s}\right)\end{array}$ & Date & $\begin{array}{c}\text { Discharge } \\
\left(\mathrm{ft}^{3} / \mathrm{s}\right)\end{array}$ & Date & $\begin{array}{c}\text { Discharge } \\
\left(\mathrm{ft}^{3} / \mathrm{s}\right)\end{array}$ & Date & $\begin{array}{c}\text { Discharge } \\
\left(\mathrm{ft}^{3} / \mathrm{s}\right)\end{array}$ \\
\hline $\begin{array}{r}9-10-51 \\
11-27-51 \\
1-16-52 \\
3-5-52 \\
4-23-52 \\
6-11-52 \\
7-22-52 \\
9-3-52 \\
10-9-52 \\
11-18-52 \\
12-28-52 \\
2-4-53 \\
3-10-53 \\
4-14-53 \\
6-2-53 \\
6-23-53 \\
8-19-53 \\
9-30-53 \\
11-17-53 \\
1-12-54 \\
2-25-54 \\
4-20-54 \\
5-28-54\end{array}$ & $\begin{array}{c}28.6 \\
16.1 \\
13.6 \\
15.1 \\
124 . \\
25.7 \\
10.1 \\
9.93 \\
8.20 \\
7.41 \\
8.95 \\
7.48 \\
8.99 \\
13.4 \\
23.1 \\
15.4 \\
20.4 \\
13.9 \\
10.8 \\
10.2 \\
1.3 .8 \\
7.74 \\
52.3\end{array}$ & $\begin{array}{r}7-14-54 \\
8-24-54 \\
9-16-54 \\
11-23-54 \\
12-21-54 \\
2-1-55 \\
3-2-55 \\
6-13-55 \\
7-27-55 \\
9-21-55 \\
1-17-58 \\
3-19-58 \\
6-11-58 \\
9-5-58 \\
11-5-58 \\
1-8-59 \\
7-15-59 \\
9-15-59 \\
10-27-59 \\
12-8-59 \\
1-27-60 \\
3-23-60\end{array}$ & $\begin{array}{l}17.0 \\
10.6 \\
6.73 \\
9.65 \\
10.3 \\
18.8 \\
23.4 \\
20.4 \\
21.6 \\
12.0 \\
38.8 \\
56.5 \\
37.6 \\
17.8 \\
9.94 \\
10.8 \\
21.2 \\
7.07 \\
25.7 \\
29.8 \\
51.6 \\
48.9\end{array}$ & $\begin{array}{r}5-3-60 \\
8-25-60 \\
12-1-60 \\
1-12-61 \\
3-1-61 \\
4-5-61 \\
5-17-61 \\
7-19-61 \\
11-30-61 \\
8-22-62 \\
7-22-63 \\
10-11-63 \\
12-16-64 \\
3-4-65 \\
6-18-65 \\
11-30-65 \\
3-17-66 \\
6-30-66 \\
11-8-66 \\
2-20-67 \\
6-6-67 \\
8-7-67\end{array}$ & $\begin{array}{l}41.6 \\
18.8 \\
17.4 \\
27.1 \\
22.1 \\
36.0 \\
29.5 \\
10.6 \\
44.2 \\
13.0 \\
7.71 \\
8.61 \\
41.8 \\
36.0 \\
41.5 \\
10.0 \\
21.0 \\
18.0 \\
11.0 \\
6.3 \\
46.0 \\
25.0\end{array}$ & $\begin{array}{r}11-24-67 \\
4-17-68 \\
4-25-68 \\
9-19-68 \\
12-18-68 \\
3-13-69 \\
6-14-69 \\
9-17-69 \\
12-17-69 \\
2-19-70 \\
5-19-70 \\
8-4-70 \\
8-11-70 \\
12-8-70 \\
3-10-71 \\
4-8-71 \\
8-3-71 \\
8-11-71 \\
11-24-71 \\
12-11-71 \\
9-25-72 \\
5-15-73\end{array}$ & $\begin{array}{r}33.0 \\
47.0 \\
69.0 \\
22.0 \\
41.0 \\
64.0 \\
116.0 \\
23.0 \\
22.0 \\
22.0 \\
47.0 \\
21.0 \\
18.0 \\
35.0 \\
24.0 \\
17.0 \\
11.0 \\
10.0 \\
20.0 \\
161.0 \\
9.2 \\
83 .\end{array}$ \\
\hline
\end{tabular}


Pennington Creek near Tishomingo, Oklahoma, Miscellaneous site.

Lat. $34^{\circ} 19^{\prime} 50^{\prime \prime}$, long. $96^{\circ} 43^{\prime} 25^{\prime \prime}$, SW $1 / 4$ sec.5, T.3 S., R. 6 E., at ford, 6 miles northwest of Tishomingo, Oklahoma.

\begin{tabular}{|c|c|c|c|c|c|c|c|}
\hline Date & $\begin{array}{c}\text { Discharge } \\
\left(\mathrm{ft}^{3} / \mathrm{s}\right)\end{array}$ & Date & $\begin{array}{c}\text { Discharge } \\
\left(\mathrm{ft}^{3} / \mathrm{s}\right)\end{array}$ & Date & $\begin{array}{c}\text { Discharge } \\
\left(\mathrm{ft}^{3} / \mathrm{s}\right)\end{array}$ & Date & $\begin{array}{c}\text { Discharge } \\
\left(\mathrm{ft}^{3} / \mathrm{s}\right)\end{array}$ \\
\hline $7-19-49$ & 25.0 & $8-30-49$ & 17.0 & $6-30-50$ & 45.6 & $11-1-50$ & 40.4 \\
\hline
\end{tabular}

Persimmon Creek near Mutual, Oklahoma, 07237700 , partial record site.

Lat. $36^{\circ} 15^{\prime} 45^{\prime \prime}$, long. $99^{\circ} 10^{\prime} 30^{\prime \prime}$, in $\mathrm{NW} 1 / 4 \mathrm{NW} 1 / 4 \mathrm{sec} .31, \mathrm{~T} .21 \mathrm{~N} ., \mathrm{R} .18 \mathrm{~W}$., Woodward County, at bridge on U.S. Highway 270,2 miles north of Mutual, and 5 miles upstream from mouth.

\begin{tabular}{|c|c|c|c|c|c|c|c|}
\hline Date & $\begin{array}{c}\text { Discharge } \\
\left(\mathrm{ft}^{3} / \mathrm{s}\right)\end{array}$ & Date & $\begin{array}{c}\text { Discharge } \\
\left(\mathrm{ft}^{3} / \mathrm{s}\right)\end{array}$ & Date & $\begin{array}{c}\text { Discharge } \\
\left(\mathrm{ft}^{3} / \mathrm{s}\right)\end{array}$ & Date & $\begin{array}{c}\text { Discharge } \\
\left(\mathrm{ft}^{3} / \mathrm{s}\right)\end{array}$ \\
\hline $2-20-44$ & 7.75 & $2-17-65$ & 7.88 & $10-19-67$ & 0 & $7-24-70$ & $\begin{array}{l}0 \\
0\end{array}$ \\
\hline $\begin{array}{r}11-25-44 \\
9-28-49\end{array}$ & $\begin{array}{l}5.80 \\
3.58\end{array}$ & $\begin{array}{l}4-28-65 \\
7-21-65\end{array}$ & $\begin{array}{l}4.41 \\
0\end{array}$ & $\begin{array}{l}2-8-68 \\
6-20-68\end{array}$ & $\begin{array}{l}4.7 \\
2.1\end{array}$ & $\begin{array}{r}11-5-70 \\
3-4-71\end{array}$ & $\begin{array}{l}0 \\
13.0\end{array}$ \\
\hline $10-29-57$ & $\begin{array}{l}3.58 \\
3.54\end{array}$ & $10-7-65$ & $\begin{array}{l}0 \\
0\end{array}$ & $\begin{array}{l}0-20-08 \\
8-9-68\end{array}$ & 0 & $5-27-71$ & 13.0 \\
\hline $1-8-58$ & 3.01 & $2-16-66$ & 11.0 & $11-21-68$ & 0 & $9-8-71$ & 0 \\
\hline $2-5-58$ & 5.81 & $3-8-66$ & 8.5 & $3-13-69$ & 15. & $12-16-71$ & 6.4 \\
\hline $3-31-58$ & 15.6 & $4-13-66$ & 4.7 & $6-25-69$ & 4.7 & $2-17-72$ & 4.7 \\
\hline $5-19-58$ & 4.33 & $7-7-66$ & 0 & $8-5-69$ & 0 & $5-25-72$ & 0 \\
\hline $7-24-58$ & 16.1 & $12-1-66$ & 1.1 & $9-26-60$ & 1.0 & $8-15-72$ & .4 \\
\hline $10-21-58$ & 0 & $2-2-67$ & 6.4 & $12-18-69$ & 4.9 & $12-21-72$ & 11. \\
\hline $6-15-59$ & 0 & $4-27-67$ & 5.8 & $2-26-70$ & 4.6 & $3-1-73$ & 0.6 \\
\hline $8-13-59$ & 0 & $7-27-67$ & 0 & $5-13-70$ & 2.9 & $5-22-73$ & 7.9 \\
\hline $10-29-64$ & 0 & & & & & & \\
\hline
\end{tabular}

Persimmon Creek near Sharon, Oklahoma, Miscellaneous site.

Lat. $36^{\circ} 15^{\prime} 28^{\prime \prime}$, long. $99^{\circ} 12^{\prime} 51^{\prime \prime}, \sec 33$, T.21 N., R.19 w., 6 miles east of Sharon, Okla.

\begin{tabular}{|c|c|c|c|c|c|c|c|}
\hline Date & $\begin{array}{c}\text { Discharge } \\
\left(\mathrm{ft}^{3} / \mathrm{s}\right)\end{array}$ & Date & $\begin{array}{c}\text { Discharge } \\
\left(\mathrm{ft}^{3} / \mathrm{s}\right)\end{array}$ & Date & $\begin{array}{c}\text { Discharge } \\
\left(\mathrm{ft}^{3} / \mathrm{s}\right)\end{array}$ & Date & $\begin{array}{c}\text { Discharge } \\
\left(\mathrm{ft}^{3} / \mathrm{s}\right)\end{array}$ \\
\hline $\begin{array}{l}3-15-56 \\
4-17-56 \\
5-25-56\end{array}$ & $\begin{array}{r}1.46 \\
1.37 \\
.11\end{array}$ & $\begin{array}{l}6-18-56 \\
2-27-57\end{array}$ & 0.78 & $\begin{array}{l}4-11-57 \\
7-10-57\end{array}$ & $\begin{array}{l}5.50 \\
3.41\end{array}$ & $\begin{array}{l}8-14-57 \\
9-11-57\end{array}$ & $\begin{array}{l}.08 \\
.42\end{array}$ \\
\hline
\end{tabular}

Persimmon Creek (North), near Sharon, Oklahoma, Miscellaneous site.

Lat. $36^{\circ} 20^{\prime} 00^{\prime \prime}$, long. $99^{\circ} 20^{\prime} 00^{\prime \prime}$ on west line of sec.27, T.21 N., R.20 W., at bridge on State Highway $34,1 / 2$ mile south of Sharon, Okla.

\begin{tabular}{|c|c|c|c|c|c|c|c|}
\hline Date & $\begin{array}{c}\text { Discharge } \\
\left(\mathrm{ft}^{3} / \mathrm{s}\right)\end{array}$ & Date & $\begin{array}{c}\text { Discharge } \\
\left(\mathrm{ft}^{3} / \mathrm{s}\right)\end{array}$ & Date & $\begin{array}{c}\text { Discharge } \\
\left(\mathrm{ft}^{3} / \mathrm{s}\right)\end{array}$ & Date & $\begin{array}{c}\text { Discharge } \\
\left(\mathrm{ft}^{3} / \mathrm{s}\right)\end{array}$ \\
\hline $\begin{array}{l}3-15-56 \\
4-17-56 \\
5-23-56 \\
6-19-56\end{array}$ & $\begin{array}{r}1.49 \\
1.15 \\
.64 \\
.27\end{array}$ & $\begin{array}{r}12-12-56 \\
2-27-57 \\
4-11-57\end{array}$ & $\begin{array}{r}.77 \\
2.90 \\
2.82\end{array}$ & $\begin{array}{l}7-10-57 \\
8-14-57 \\
9-11-57\end{array}$ & $\begin{array}{r}1.57 \\
.38 \\
1.51\end{array}$ & $\begin{array}{l}10-18-57 \\
11-12-57 \\
12-20-57\end{array}$ & $\begin{array}{l}2.29 \\
2.85 \\
2.81\end{array}$ \\
\hline
\end{tabular}

Persimmon Creek (South), near Sharon, Oklahoma, Miscellaneous site.

Lat. $36^{\circ} 13^{\prime} 30^{\prime \prime}$, long. $99^{\circ} 19^{\prime} 00^{\prime \prime}$, on north line of sec.10, T.20 N., R.20 K., at county highway bridge, 3 miles south of Sharon, Okla.

\begin{tabular}{|c|c|c|c|c|c|c|c|}
\hline Date & $\begin{array}{c}\text { Discharge } \\
\left(\mathrm{ft}^{3} / \mathrm{s}\right)\end{array}$ & Date & $\begin{array}{c}\text { Discharge } \\
\left(\mathrm{ft}^{3} / \mathrm{s}\right)\end{array}$ & Date & $\begin{array}{c}\text { Discharge } \\
\left(\mathrm{ft}^{3} / \mathrm{s}\right)\end{array}$ & Date & $\begin{array}{c}\text { Discharge } \\
\left(\mathrm{ft}^{3} / \mathrm{s}\right)\end{array}$ \\
\hline $\begin{array}{l}3-15-56 \\
4-17-56 \\
5-23-56 \\
6-19-56\end{array}$ & $\begin{array}{r}1.61 \\
.94 \\
.19 \\
0\end{array}$ & $\begin{array}{r}12-12-56 \\
2-27-57 \\
4-11-57\end{array}$ & $\begin{array}{r}.13 \\
1.48 \\
2.34\end{array}$ & $\begin{array}{l}7-10-57 \\
8-14-57 \\
9-11-57\end{array}$ & $\begin{array}{l}0^{.23} \\
0\end{array}$ & $\begin{array}{l}10-18-57 \\
11-13-57 \\
12-20-57\end{array}$ & $\begin{array}{l}1.52 \\
2.56 \\
2.46\end{array}$ \\
\hline
\end{tabular}


Pigeon Creek near Page, Oklahoma, Miscellaneous site.

Lat. $34^{\circ} 39^{\prime} 36^{\prime \prime}$, long. $94^{\circ} 31^{\prime} 25^{\prime \prime}$, near center sec.14, T.2 N., R.26 E., at bridge crossing of State Highway 63, 4-1/2 miles south of Page, Okla.

$\begin{array}{rr}\text { Date } & \begin{array}{r}\text { Discharge } \\ \left(\mathrm{ft}^{3} / \mathrm{s}\right)\end{array} \\ 7-10-60 & 1.08\end{array}$

Pond Creek near Eakly, Oklahoma, Miscellaneous site.

Lat. $35^{\circ} 17^{\prime} 46^{\prime \prime}$, long. $98^{\circ} 37^{\prime} 25^{\prime \prime}$, center E line sec.31, T.10 N., R.13 W., at county highway bridge, 3 miles west of Eakly, Okla.

\begin{tabular}{|c|c|c|c|c|c|c|c|}
\hline Date & $\begin{array}{c}\text { Di scharge } \\
\left(\mathrm{ft}^{3} / \mathrm{s}\right)\end{array}$ & Date & $\begin{array}{c}\text { Discharge } \\
\left(\mathrm{ft}^{3} / \mathrm{s}\right)\end{array}$ & Date & $\begin{array}{c}\text { Discharge } \\
\left(\mathrm{ft}^{3} / \mathrm{s}\right)\end{array}$ & Date & $\begin{array}{c}\text { Discharge } \\
\left(\mathrm{ft}^{3} / \mathrm{s}\right)\end{array}$ \\
\hline 52 & 2.98 & $11-5-52$ & 5.20 & $4-30-53$ & 6.14 & $6-22-53$ & 2.22 \\
\hline
\end{tabular}

Pond Creek near Fort Cobb, Oklahoma, Miscellaneous site.

Lat. $35^{\circ} 21^{\prime} 55^{\prime \prime}$, long. $98^{\circ} 30^{\prime} 17^{\prime \prime}$, NW $1 / 4$ sec. 30 , T. 9 N., R. 12 w., at county highway bridge $1 / 4 \mathrm{mile}$ south of Swan Lake and 10 miles northwest of Fort Cobb, Oklahoma.

$\begin{array}{cccccc}\text { Date } & \begin{array}{c}\text { Discharge } \\ \left(\mathrm{ft}^{3} / \mathrm{s}\right)\end{array} & \text { Date } & \begin{array}{c}\text { Discharge } \\ \left(\mathrm{ft}^{3} / \mathrm{s}\right)\end{array} & \text { Date } & \begin{array}{c}\text { Discharge } \\ \left(\mathrm{ft}^{3} / \mathrm{s}\right)\end{array} \\ 10-23-46 & 17.8 & 10-23-46 & 21.3 & 10-23-46 & 22.5\end{array}$

Pond Creek near Swan Lake, Oklahoma, Miscellaneous site.

Lat. $35^{\circ} 15^{\prime} 55^{\prime \prime}$, long. $98^{\circ} 37^{\prime} 36^{\prime \prime}$, SE $1 / 4$ sec.22, T.9 N., R.13 w., at county highway bridge, 2 miles west of Swan Lake, Okla.

$\begin{array}{lrcrcrrr}\text { Date } & \begin{array}{c}\text { Discharge } \\ \left(\mathrm{ft}^{3} / \mathrm{s}\right)\end{array} & \text { Date } & \begin{array}{c}\text { Discharge } \\ \left(\mathrm{ft}^{3} / \mathrm{s}\right)\end{array} & \text { Date } & \begin{array}{c}\text { Discharge } \\ \left(\mathrm{ft}^{3} / \mathrm{s}\right)\end{array} & \text { Date } & \begin{array}{c}\text { Discharge } \\ \left(\mathrm{ft}^{3} / \mathrm{s}^{3}\right)\end{array} \\ 9-15-52 & 4.56 & 11-5-52 & 8.93 & 4-30-53 & 12.3 & 6-22-53 & 3.67\end{array}$

Pond Creek near Lamont, Oklahoma, Miscellaneous site.

Lat. $36^{\circ} 41^{\prime} 00^{\prime \prime}$, long. $97^{\circ} 35^{\prime} 30^{\prime \prime}$, SE $1 / 4 \mathrm{sec} .35$, T. $26 \mathrm{~N}$, R. R w., at ford $1-3 / 4$ miles above St. Louis 8

San Francisco Railway bridge and 2 miles southwest of Lamont, Oklahoma.

\begin{tabular}{|c|c|c|c|c|c|c|c|}
\hline Date & $\begin{array}{c}\text { - Discharge } \\
\left(\mathrm{ft}^{3} / \mathrm{s}\right)\end{array}$ & Date & $\begin{array}{c}\text { Discharge } \\
\left(\mathrm{ft}^{3} / \mathrm{s}\right)\end{array}$ & Date & $\begin{array}{c}\text { Discharge } \\
\left(\mathrm{ft}^{3} / \mathrm{s}\right)\end{array}$ & Date & $\begin{array}{c}\text { Discharge } \\
\left(\mathrm{ft}^{3} / \mathrm{s}\right)\end{array}$ \\
\hline $\begin{array}{r}9-19-51 \\
10-24-51 \\
10-11-51 \\
1-23-52 \\
3-4-52 \\
5-8-52 \\
6-2-52 \\
7-24-52 \\
9-3-52 \\
11-5-52\end{array}$ & $\begin{array}{r}31.50 \\
4.97 \\
11.00 \\
9.97 \\
37.40 \\
15.40 \\
3.99 \\
.51 \\
.53 \\
.32\end{array}$ & $\begin{array}{r}12-23-52 \\
2-17-53 \\
3-24-53 \\
5-5-53 \\
6-2-53 \\
6-23-53 \\
9-22-53 \\
10-22-53 \\
11-25-53\end{array}$ & $\begin{array}{r}1.17 \\
.96 \\
.49 \\
.43 \\
.14 \\
.90 \\
.10 \\
.10 \\
2.21\end{array}$ & $\begin{array}{r}12-28-53 \\
1-12-54 \\
2-16-54 \\
3-16-54 \\
4-6-54 \\
6-7-54 \\
7-7-54 \\
8-3-54 \\
9-29-54\end{array}$ & $\begin{array}{r}.60 \\
.24 \\
.18 \\
.19 \\
.59 \\
2.13 \\
.05 \\
.05 \\
.02\end{array}$ & $\begin{array}{r}10-25-54 \\
11-4-54 \\
1-4-55 \\
2-1-55 \\
3-22-55 \\
4-5-55 \\
5-3-55 \\
7-13-55 \\
8-2-55 \\
9-21-55\end{array}$ & $\begin{array}{l}.06 \\
.05 \\
.09 \\
.09 \\
.13 \\
.36 \\
.05 \\
.05 \\
.01 \\
.01\end{array}$ \\
\hline
\end{tabular}

Pony Creek near Optima, Oklahoma, 07232620 , partial record site.

Lat. $36^{\circ} 45^{\prime} 39^{\prime \prime}$, long. 101 $1^{\circ} 7^{\prime} 26^{\prime \prime}$, on south line sec. 36 , T.4 N., R. 16 E., Texas County, at county road bridge, 4 miles east of Optima, Okla.

\begin{tabular}{|c|c|c|c|c|c|c|c|}
\hline Date & $\begin{array}{c}\text { Discharge } \\
\left(\mathrm{ft}^{3} / \mathrm{s}\right)\end{array}$ & Date & $\begin{array}{c}\text { Discharge } \\
\left(\mathrm{ft}^{3} / \mathrm{s}\right)\end{array}$ & Date & $\begin{array}{c}\text { Discharge } \\
\left(\mathrm{ft}^{3} / \mathrm{s}\right)\end{array}$ & Date & $\begin{array}{c}\text { Dischargle } \\
\left(\mathrm{ft}^{3} / \mathrm{s}\right)\end{array}$ \\
\hline $\begin{array}{r}11-23-66 \\
1-23-67 \\
5-8-67 \\
8-31-67 \\
11-27-67\end{array}$ & $\begin{array}{l}0 \\
0 \\
0 \\
0 \\
0\end{array}$ & $\begin{array}{r}3-22-68 \\
4-18-68 \\
8-9-68 \\
11-22-68 \\
2-28-69\end{array}$ & $\begin{array}{l}0 \\
0 \\
0 \\
0 \\
0\end{array}$ & $\begin{array}{r}5-26-69 \\
8-12-69 \\
12-24-69 \\
3-9-70 \\
5-5-70\end{array}$ & $\begin{array}{l}0 \\
0 \\
0 \\
0 \\
0\end{array}$ & $\begin{array}{r}8-7-70 \\
12-2-70 \\
3-5-71 \\
6-15-71 \\
9-3-71\end{array}$ & $\begin{array}{l}0 \\
0 \\
0 \\
0 \\
0\end{array}$ \\
\hline
\end{tabular}


Pryor Creek near Pryor, Oklahoma, Miscellaneous site.

Lat. $36^{\circ} 15^{\prime} 00^{\prime \prime}$, long. $95^{\circ} 15^{\prime} 30^{\prime \prime}$, NE $1 / 4$ sec.3, T.20 N., R. 19 E., at site below paper mill, 5 miles southeast of Pryor, Okla.

\begin{tabular}{|c|c|c|c|c|c|c|c|}
\hline Date & $\begin{array}{c}\text { Discharge } \\
\left(\mathrm{ft}^{3} / \mathrm{s}\right)\end{array}$ & Date & $\begin{array}{c}\text { Discharge } \\
\left(\mathrm{ft}^{3} / \mathrm{s}\right)\end{array}$ & Date & $\begin{array}{c}\text { Discharge } \\
\left(\mathrm{ft}^{3} / \mathrm{s}\right)\end{array}$ & Date & $\begin{array}{c}\text { Discharge } \\
\left(\mathrm{ft}^{3} / \mathrm{s}\right)\end{array}$ \\
\hline $\begin{array}{r}4-11-52 \\
6-2-52 \\
8-7-52 \\
8-13-52 \\
10-23-52 \\
12-5-52 \\
2-13-53\end{array}$ & $\begin{array}{c}52.8 \\
10.2 \\
13.2 \\
13.2 \\
6.02 \\
7.94 \\
12.4\end{array}$ & $\begin{array}{l}5-15-53 \\
6-18-53 \\
7-23-53 \\
8-18-53 \\
9-30-53 \\
8-19-54\end{array}$ & $\begin{array}{r}138.0 \\
12.0 \\
10.6 \\
10.8 \\
8.42 \\
.40\end{array}$ & $\begin{array}{r}4-12-54 \\
10-28-53 \\
11-25-53 \\
12-17-53 \\
1-29-54 \\
2-10-54\end{array}$ & $\begin{array}{l}3.52 \\
13.6 \\
14.6 \\
8.98 \\
9.62 \\
8.04\end{array}$ & $\begin{array}{l}3-11-54 \\
4-12-54 \\
5-18-54 \\
6-16-54 \\
8-19-54 \\
9-29-54\end{array}$ & $\begin{array}{c}7.37 \\
14.2 \\
7.62 \\
17.6 \\
9.16 \\
10.2\end{array}$ \\
\hline
\end{tabular}

Quapaw Creek near Meeker, Oklahoma, Miscellaneous site.

Lat. $35^{\circ} 31^{\prime} 00^{\prime \prime}$, long. $96^{\circ} 52^{\prime} 00^{\prime \prime}$, NW $1 / 4 \mathrm{NW} 1 / 4$ sec.if, T.12 N., R.4 E., at hridge on State Highway 18 , 1 mile north of Meeker, okla.

\begin{tabular}{|c|c|c|c|c|c|c|c|}
\hline Date & $\begin{array}{c}\text { Discharge } \\
\left(\mathrm{ft}^{3} / \mathrm{s}\right)\end{array}$ & Date & $\begin{array}{c}\text { Discharge } \\
\left(\mathrm{ft}^{3} / \mathrm{s}\right)\end{array}$ & Date & $\begin{array}{c}\text { Discharge } \\
\left(\mathrm{ft}^{3} / \mathrm{s}\right)\end{array}$ & Date & $\begin{array}{c}\text { Discharge } \\
\left(\mathrm{ft}^{3} / \mathrm{s}\right)\end{array}$ \\
\hline $\begin{array}{r}11-17-53 \\
12-16-53 \\
2-2-54\end{array}$ & $\begin{array}{r}0 \\
1.16 \\
.90\end{array}$ & $\begin{array}{l}3-23-54 \\
5-4-54 \\
6-14-54\end{array}$ & $\begin{aligned} 26.99 \\
.10\end{aligned}$ & $\begin{array}{r}8-20-54 \\
11-18-54 \\
2-15-55\end{array}$ & $\begin{array}{l}0 \\
0 \\
0\end{array}$ & $\begin{array}{l}4-28-55 \\
7-11-55 \\
8-17-55\end{array}$ & $\begin{array}{l}8.42 \\
0 \\
0\end{array}$ \\
\hline
\end{tabular}

Rainy Mountain Creek near Mountain View, Oklahoma, 07325300 , partial record site.

Lat. $35^{\circ} 05^{\prime} 45^{\prime \prime}$, 1ong. $98^{\circ} 43^{\prime} 00^{\prime \prime}$, NW $1 / 4$ sec.12, T.7 N., R.15 W., at bridge on State Highnay 9 , $1 / 4$ mile east of Mountain View, Oklá.

\begin{tabular}{|c|c|c|c|c|c|c|c|}
\hline Date & $\begin{array}{c}\text { Discharge } \\
\left(\mathrm{ft}^{3} / \mathrm{s}\right)\end{array}$ & Date & $\begin{array}{c}\text { Discharge } \\
\left(\mathrm{ft}^{3} / \mathrm{s}\right)\end{array}$ & Date & $\begin{array}{c}\text { Discharge } \\
\left(\mathrm{ft}^{3} / \mathrm{s}\right)\end{array}$ & Date & $\begin{array}{c}\text { Discharge } \\
\left(\mathrm{ft}^{3} / \mathrm{s}\right)\end{array}$ \\
\hline $\begin{array}{r}9-5-51 \\
10-8-51 \\
11-29-51 \\
1-15-52 \\
3-5-52 \\
5-16-52 \\
6-4-52 \\
7-9-52 \\
8-20-52 \\
10-1-52 \\
10-7-52 \\
12-11-52\end{array}$ & $\begin{array}{l}0 \\
11.9 \\
.15 \\
.09 \\
.38 \\
0 \\
8.57 \\
0 \\
0 \\
0 \\
0 \\
0\end{array}$ & $\begin{array}{r}1-20-53 \\
3-2-53 \\
3-31-53 \\
5-18-53 \\
6-22-53 \\
7-27-53 \\
8=25-53 \\
11-3-53 \\
12-1-53 \\
1-18-54 \\
2-15-54 \\
3-15-54\end{array}$ & $\begin{array}{l}0 \\
.02 \\
0.21 \\
0 \\
.1 \\
0 \\
1.07 \\
.04 \\
.04 \\
.04 \\
.04\end{array}$ & $\begin{array}{r}5-5-54 \\
6-3-54 \\
6-29-54 \\
8-2-54 \\
9-7-54 \\
10-19-54 \\
11-15-54 \\
12-16-54 \\
1-17-55 \\
2-14-55 \\
3-16-55 \\
4-19-55\end{array}$ & $\begin{array}{l}6.09 \\
5.78 \\
0 \\
0 \\
0 \\
0 \\
0 \\
0 \\
0 \\
0 \\
0 \\
0\end{array}$ & $\begin{array}{r}6-15-55 \\
7-20-55 \\
8-22-55 \\
2-4-58 \\
2-24-59 \\
8-21-59 \\
9-15-59 \\
11-30-60 \\
3-7-61 \\
3-15-61 \\
6-2-61\end{array}$ & $\begin{array}{l}3.33 \\
15.4 \\
0 \\
8.93 \\
1.93 \\
.02 \\
0 \\
4.54 \\
4.05 \\
3.34 \\
0\end{array}$ \\
\hline
\end{tabular}

Red Rock Creek near Dougherty, Oklahoma, Miscellaneous site. Lat. $34^{\circ} 24^{\prime} 28^{\prime \prime}$, long. $97^{\circ} 0 \mathrm{I}^{\prime} 43^{\prime \prime}$, SW $1 / 4 \mathrm{sec} .7, \mathrm{~T} .2 \mathrm{~S} ., \mathrm{R} .3 \mathrm{E}$. , at county highway bridge, 1 mile east
of Dougherty, okla.

Date $\quad \begin{gathered}\text { Discharge } \\ \left(\mathrm{ft}^{3} / \mathrm{s}\right)\end{gathered}$

$10-7.52 \quad 6.39$ 
Red Rock Creek near Red Rock, Oklahoma, Miscellaneous site.

Lat. $36^{\circ} 30^{\prime} 00^{\prime \prime}$, long. $97^{\circ} 10^{\prime} 00^{\prime \prime}, \mathrm{SW}^{\prime} 1 / 4 \mathrm{sec} .15, \mathrm{~T} .23 \mathrm{~N}$., R.1 E., at bridge on State Highway $15,1 / 2$ mile west of Red Rock, Okla.

\begin{tabular}{|c|c|c|c|c|c|c|c|}
\hline Date & $\begin{array}{c}\text { Discharge } \\
\left(\mathrm{ft}^{3} / \mathrm{s}\right)\end{array}$ & Date & $\begin{array}{c}\text { Discharge } \\
\left(\mathrm{ft}^{3} / \mathrm{s}\right)\end{array}$ & Date & $\begin{array}{c}\text { Di scharge } \\
\left(\mathrm{ft}^{3} / \mathrm{s}\right)\end{array}$ & Date & $\begin{array}{c}\text { Discharge } \\
\left(\mathrm{ft}^{3} / \mathrm{s}\right)\end{array}$ \\
\hline $\begin{array}{r}9-4-51 \\
10-23-51 \\
12-11-51 \\
1-22-52 \\
3-4-52 \\
6-2-52 \\
9-3-52 \\
1-7-53 \\
2-3-53 \\
3-24-53 \\
5-6-53 \\
6-2-53 \\
6-23-53 \\
8-25-53 \\
9-22-53\end{array}$ & $\begin{array}{r}.87 \\
26.30 \\
8.75 \\
8.61 \\
101.00 \\
2.86 \\
0 \\
0 \\
0 \\
.13 \\
.03 \\
.02 \\
.30 \\
.03 \\
0\end{array}$ & $\begin{array}{r}10-22-53 \\
11-25-53 \\
12-15-53 \\
1-12-54 \\
2-16-54 \\
3-2-54 \\
4-6-54 \\
6-7-54 \\
8-3-54 \\
9-8-54 \\
10-12-54 \\
11-15-54 \\
12-15-54 \\
1-4-55\end{array}$ & $\begin{array}{l}0 \\
2.30 \\
.16 \\
.01 \\
0 \\
.03 \\
.08 \\
.65 \\
0 \\
0 \\
64.3 \\
0 \\
0 \\
0\end{array}$ & $\begin{array}{r}2-8-55 \\
3-22-55 \\
4-5-55 \\
5-3-55 \\
6-27-55 \\
8-2-55 \\
9-21-55 \\
10-27-55 \\
11-14-55 \\
12-20-55 \\
1-6-56 \\
2-21-56 \\
3-8-56 \\
4-13-56\end{array}$ & $\begin{array}{l}0 \\
3.53 \\
.32 \\
0 \\
8.26 \\
0 \\
0 \\
1.12 \\
.85 \\
.98 \\
.27 \\
1.33 \\
.43 \\
3.95\end{array}$ & $\begin{array}{r}5-9-56 \\
6-5-56 \\
7-3-56 \\
8-15-56 \\
11-14-56 \\
12-11-56 \\
1-3-57 \\
2-5-57 \\
3-6-57 \\
4-11-57 \\
5-1-57 \\
7-25-57 \\
8-8-57 \\
9-10-57 \\
10-22-57\end{array}$ & $\begin{array}{c}.26 \\
.69 \\
0.86 \\
.10 \\
0.10 \\
0 \\
0 \\
1.38 \\
32.1 \\
31.4 \\
3.60 \\
1.13 \\
2.27\end{array}$ \\
\hline
\end{tabular}

Rock Creek near Dougherty, Oklahoma, 07329900 , partial record site.

Lat. $34^{\circ} 30^{\prime} 00^{\prime \prime}$, long. $97^{\circ} 05^{\prime} 00^{\prime \prime}, W^{\prime} 1 / 2 \mathrm{sec} .7, \mathrm{~T} .2 \mathrm{~S} ., \mathrm{R} .3 \mathrm{E} ., 1 \mathrm{mile}$ east of Dougherty, Oklahoma.

\begin{tabular}{|c|c|c|c|c|c|c|c|}
\hline Date & $\begin{array}{c}\text { Discharge } \\
\left(\mathrm{ft}^{3} / \mathrm{s}\right)\end{array}$ & Date & $\begin{array}{c}\text { Discharge } \\
\left(\mathrm{ft}^{3} / \mathrm{s}\right)\end{array}$ & Date & $\begin{array}{c}\text { Discharge } \\
\left(\mathrm{ft}^{3} / \mathrm{s}\right)\end{array}$ & Date & $\begin{array}{c}\text { Discharge } \\
\left(\mathrm{ft}^{3} / \mathrm{s}\right)\end{array}$ \\
\hline $\begin{array}{r}11-30-49 \\
2-8-50 \\
9-10-51 \\
10-10-51 \\
11-27-51 \\
1-16-52 \\
3-5-52 \\
4-22-52 \\
6-12-52 \\
7-24-52\end{array}$ & $\begin{array}{c}14.8 \\
32.6 \\
28.2 \\
9.19 \\
14.7 \\
10.8 \\
17.5 \\
534.0 \\
13.5 \\
5.48\end{array}$ & $\begin{array}{r}9-2-52 \\
11-18-52 \\
12-23-52 \\
2-4-53 \\
3-10-53 \\
4-14-53 \\
6-2-53 \\
6-23-53 \\
8-19-53 \\
10-1-53\end{array}$ & $\begin{array}{c}3.30 \\
14.9 \\
10.4 \\
8.23 \\
13.7 \\
6.92 \\
13.2 \\
5.74 \\
15.5 \\
5.43\end{array}$ & $\begin{array}{r}11-17-53 \\
1-12-54 \\
2-24-54 \\
4-19-54 \\
5-27-54 \\
7-13-54 \\
8-23-54 \\
9-15-54 \\
11-1-54 \\
12-7-54\end{array}$ & $\begin{array}{r}9.70 \\
13.1 \\
8.60 \\
6.19 \\
53.60 \\
6.90 \\
3.87 \\
2.00 \\
8.16 \\
7.20\end{array}$ & $\begin{array}{r}1-17-55 \\
-1-55 \\
4-11-55 \\
6-14-55 \\
7-27-55 \\
0-20-55 \\
7-18-58 \\
10-30-58 \\
2-19-59 \\
7-15-59\end{array}$ & $\begin{array}{c}13.3 \\
12.7 \\
.68 \\
6.27 \\
2.93 \\
.37 \\
19.2 \\
11.1 \\
7.85 \\
4.60\end{array}$ \\
\hline
\end{tabular}

Roman Nose Springs near Watonga, Oklahoma, Miscellaneous site.

Lat. $35^{\circ} 59^{\prime} 00^{\prime \prime}$, long. $98^{\circ} 25^{\circ} 00^{\prime \prime}$, SW $1 / 4$ sec. 24 , T. 17 N., R. 12 W., in Roman Nose State Park, 6 miles north of Watonga, Okiahoma.

\begin{tabular}{|c|c|c|c|c|c|c|c|}
\hline Date & $\begin{array}{c}\text { Discharge } \\
\left(\mathrm{ft}^{3} / \mathrm{s}\right)\end{array}$ & Date & $\begin{array}{c}\text { Discharge } \\
\left(\mathrm{ft}^{3} / \mathrm{s}\right)\end{array}$ & Date & $\begin{array}{c}\text { Discharge } \\
\left(\mathrm{ft}^{3} / \mathrm{s}\right)\end{array}$ & Date & $\begin{array}{c}\text { Discharge } \\
\left(\mathrm{ft}^{3} / \mathrm{s}\right)\end{array}$ \\
\hline $\begin{array}{r}9-27-49 \\
10-15-52\end{array}$ & $\begin{array}{l}1.80 \\
1.18\end{array}$ & $7-7-53$ & 1.22 & $8-17-54$ & .92 & $\begin{array}{r}9-18-55 \\
11-5-57\end{array}$ & $\begin{array}{l}1.06 \\
1.96\end{array}$ \\
\hline
\end{tabular}

Round Spring Creek near nisney, Oklahoma, Miscellaneous site.

Lat. $36^{\circ} 29^{\prime} 46^{\prime \prime}$, long. $95^{\circ} 01^{\prime} 08^{\prime \prime}$, SW $1 / 4 \mathrm{sec} .13$, T.23 N., R.21 E., at county highway bridge, $1 / 2 \mathrm{mile}$ south of Disney, Okla.

$\begin{array}{lr}\text { Date } & \begin{array}{r}\text { Discharge } \\ \left(\mathrm{ft}^{3} / \mathrm{s}\right)\end{array} \\ 3-5-54 & 2.23\end{array}$

Roundup Creek near Woodward, Oklahoma, Miscellaneous site.

Lat. $36^{\circ} 28^{\prime} 30^{\prime \prime}$, long. $99^{\circ} 28^{\prime} 30^{\prime \prime}$, NW $1 / 4$ sec.17, T. $23 \mathrm{~N} .$, R. $21 \mathrm{~W}$, at bridge on U.S. Highway 270,5 miles northwest of Woodward, Okla.

$\begin{array}{lcrrrrrr}\text { Date } & \begin{array}{c}\text { Discharge } \\ \left(\mathrm{ft}^{3} / \mathrm{s}\right)\end{array} & \text { Date } & \begin{array}{c}\text { Discharge } \\ \left(\mathrm{ft}^{3} / \mathrm{s}\right)\end{array} & \text { Date } & \begin{array}{c}\text { Discharge } \\ \left(\mathrm{ft}^{3} / \mathrm{s}\right)\end{array} & \text { Date } & \begin{array}{c}\text { Discharge } \\ \left(\mathrm{ft}^{3} / \mathrm{s}\right)\end{array} \\ 4-18-56 & 0 & 12-12-56 & .01 & 7-11-57 & .05 & 10-17-57 & .63 \\ 5-23-56 & 0 & 2-26-5.7 & .11 & 8-14-57 & .04 & 11-12-57 & 1.10 \\ 6-19-56 & 0 & 4-12-57 & .26 & 9-11-57 & .04 & 12-19-57 & .80\end{array}$


LOK'-FLOW MEASUREMENTS - - Continued.

Rush Creek near Mackie, Oklahoma, Miscellaneous site.

Lat. $34^{\circ} 42^{\prime} 41^{\prime \prime}$, long. $99^{\circ} 48^{\prime} 34^{\prime \prime}$, SW $1 / 4 \mathrm{sec} .12$, T.14 N., R. $25 \mathrm{~W}^{\circ}$, at railway bridge, 1 mile northwest of Mackie, Okla.

$\begin{array}{cccr}\text { Date } & \begin{array}{c}\text { Discharge } \\ \left(\mathrm{ft}^{3} / \mathrm{s}\right)\end{array} & \text { Date } & \begin{array}{r}\text { Discharge } \\ \left(\mathrm{ft}^{3} / \mathrm{s}\right)\end{array} \\ 9-13-55 & 0 & 3-11-57 & 1.89\end{array}$

Rush Creek near Pauls Valley, Oklahoma, Miscellaneous site.

Lat. $34^{\circ} 43^{\prime} 45^{\prime \prime}$, long. $97^{\circ}$ i5 $00^{\prime \prime}, \mathrm{SH} 1 / 4 \mathrm{sec} 18$, T.3 N., R. 1 E., at county highway bridge, $1 \mathrm{mile}$ west of Pauls Valley, Okia.

\begin{tabular}{|c|c|c|c|c|c|c|c|}
\hline Date & $\begin{array}{c}\text { Discharge } \\
\left(\mathrm{ft}^{3} / \mathrm{s}\right)\end{array}$ & Date & $\begin{array}{c}\text { Discharge } \\
\left(\mathrm{ft}^{3} / \mathrm{s}\right)\end{array}$ & Date & $\begin{array}{c}\text { Discharge } \\
\left(\mathrm{ft}^{3} / \mathrm{s}\right)\end{array}$ & Date & $\begin{array}{c}\text { Discharge } \\
\left(\mathrm{ft}^{3} / \mathrm{s}\right)\end{array}$ \\
\hline $\begin{array}{l}7-1-52 \\
7-11-52\end{array}$ & $\begin{array}{l}1.24 \\
7.29\end{array}$ & $7-23-52$ & 3.38 & $8-1-52$ & 0 & $2-4-53$ & 7.99 \\
\hline
\end{tabular}

Rush Creek near Rush Springs, Oklahoma, Miscellaneous site.

Lat. $34^{\circ} 46^{\prime} 37^{\prime \prime}$, long. $97^{\circ} 56^{\prime} 00^{\prime \prime}$, NW $1 / 4 \mathrm{NW} 1 / 4 \mathrm{sec} 33, \mathrm{~T} .4 \mathrm{~N} ., \mathrm{R} .7 \mathrm{H}$, at bridge on county road at Rush Springs, Okla.
Date Discharge
$9-23-52 \quad .24$

Salina Creek near Salina, Oklahoma, Miscellaneous site.

Lat. $36^{\circ} 17^{\prime} 30^{\prime \prime}$, long. $95^{\circ} 07^{\prime \prime} 00^{\prime \prime}$, center sec. 26, T.21 N., R. 20 E., at bridge on State Highway $82,1-1 / 2$ miles southeast of Salina, Okla.

\begin{tabular}{|c|c|c|c|c|c|c|c|}
\hline Date & $\begin{array}{c}\text { Discharge } \\
\left(\mathrm{ft}^{3} / \mathrm{s}\right)\end{array}$ & Date & $\begin{array}{c}\text { Discharge } \\
\left(\mathrm{ft}^{3} / \mathrm{s}\right)\end{array}$ & Date & $\begin{array}{c}\text { Discharge } \\
\left(\mathrm{ft}^{3} / \mathrm{s}\right)\end{array}$ & Nate & $\begin{array}{c}\text { Discharge } \\
\left(\mathrm{ft}^{3} / \mathrm{s}\right)\end{array}$ \\
\hline $\begin{array}{r}11-10-51 \\
1-15-52 \\
2-15-52 \\
3-28-52\end{array}$ & $\begin{array}{r}133.0 \\
71.3 \\
78.2 \\
68.4\end{array}$ & $\begin{array}{l}4-25-52 \\
6-11-52 \\
7-8-52\end{array}$ & $\begin{array}{c}189.0 \\
16.8 \\
6.62\end{array}$ & $\begin{array}{r}8-8-52 \\
10-24-52 \\
2-20-53\end{array}$ & $\begin{array}{r}6.94 \\
.33 \\
7.60\end{array}$ & $\begin{array}{l}3-27-53 \\
5-20-53 \\
7-23-53 \\
7-12-60\end{array}$ & $\begin{array}{c}73.00 \\
180.0 \\
5.76 \\
11.2\end{array}$ \\
\hline
\end{tabular}

Salt Creek near Dewright, Oklahoma, Miscellaneous site.

Lat. $35^{\circ} 00^{\prime} 00^{\prime \prime}$, long. $96^{\circ} 40^{\circ} 00^{\prime \prime}$, SW $1 / 4$ sec.27, T.7 N., R.6 E., at bridge on State Highway $48,2-1 / 2$ miles south of Dewright, Okla.

\begin{tabular}{|c|c|c|c|c|c|c|c|}
\hline Date & $\begin{array}{c}\text { Discharge } \\
\left(\mathrm{ft}^{3} / \mathrm{s}\right)\end{array}$ & Date & $\begin{array}{c}\text { Discharge } \\
\left(\mathrm{ft}^{3} / \mathrm{s}\right)\end{array}$ & Date & $\begin{array}{c}\text { Discharge } \\
\left(\mathrm{ft}^{3} / \mathrm{s}\right)\end{array}$ & Date & $\begin{array}{c}\text { Discharge } \\
\left(\mathrm{ft}^{3} / \mathrm{s}\right)\end{array}$ \\
\hline $\begin{array}{r}9-7-51 \\
10-31-51 \\
12-12-51 \\
1-15-52 \\
2-26-52 \\
6-3-52 \\
7-22-52 \\
9-2-52 \\
10-16-53 \\
11-18-53\end{array}$ & $\begin{array}{c}.38 \\
18.0 \\
4.80 \\
5.81 \\
23.5 \\
53.6 \\
9.83 \\
.17 \\
5.50 \\
23.8\end{array}$ & $\begin{array}{r}12-16-53 \\
2-2-54 \\
3-2-54 \\
4-20-54 \\
6-2-54 \\
7-2-54 \\
9-14-54 \\
10-26-55 \\
1-6-56\end{array}$ & $\begin{array}{c}10.7 \\
16.6 \\
13.5 \\
7.35 \\
20.3 \\
2.24 \\
.92 \\
3.87 \\
5.36\end{array}$ & $\begin{array}{r}2-13-56 \\
3-15-56 \\
5-14-56 \\
7-13-56 \\
8-14-56 \\
8-27-56 \\
9-29-56 \\
11-13-56 \\
2-5-57\end{array}$ & $\begin{array}{l}6.31 \\
5.22 \\
3.83 \\
3.65 \\
0 \\
0 \\
0 \\
1.78 \\
4.44\end{array}$ & $\begin{array}{r}7-10-57 \\
9-4-57 \\
12-3-57 \\
9-17-58 \\
11-19-58 \\
12-17-58 \\
2-16-59 \\
3-31-59 \\
6-15-59 \\
8-13-59\end{array}$ & $\begin{array}{c}15.2 \\
3.48 \\
19.3 \\
11.6 \\
5.46 \\
9.71 \\
5.11 \\
8.67 \\
4.46 \\
2.56\end{array}$ \\
\hline
\end{tabular}


Salt Creek near Hitchcock, Oklahoma, Miscellaneous site.

Lat. $36^{\circ} 00^{\prime} 28^{\prime \prime}$, long. $98^{\circ} 23^{\prime} 25^{\prime \prime}$, NE $1 / 4 \mathrm{NE} 1 / 4 \mathrm{sec} .28$, T. $18 \mathrm{~N}$, R.11 W., at county highway bridge, $3-1 / 2$ miles northwest of Hitchcock, Okla.

$\begin{array}{cc}\text { Date } & \begin{array}{c}\text { Discharge } \\ \left(\mathrm{ft}^{3} / \mathrm{s}\right)\end{array} \\ 8-17-54 & 0\end{array}$

Salt Creek near Pearson, Oklahoma, Miscellaneous site.

Lat. $35^{\circ} 05^{\prime} 00^{\prime \prime}$, long. $96^{\circ} 55^{\prime} 00^{\prime \prime}$, NE $1 / 4 \mathrm{sec} .18$, T.7 N., R.4 E., at bridge on State Highway $18,1 \mathrm{mile}$ north of Pearson, Okla.

\begin{tabular}{|c|c|c|c|c|c|c|c|}
\hline Date & $\begin{array}{l}\text { Discharge } \\
\left(\mathrm{ft}^{3} / \mathrm{s}\right)\end{array}$ & Date & $\begin{array}{c}\text { Dis charge } \\
\left(\mathrm{ft}^{3} / \mathrm{s}\right)\end{array}$ & Date & $\begin{array}{c}\text { Discharge } \\
\left(\mathrm{ft}^{3} / \mathrm{s}\right)\end{array}$ & Date & $\begin{array}{c}\text { Discharge } \\
\left(\mathrm{ft}^{3} / \mathrm{s}\right)\end{array}$ \\
\hline $\begin{array}{l}2-13-56 \\
3-26-56 \\
4-17-56 \\
4-24-56 \\
5-23-56 \\
6-14-56 \\
7-3-56 \\
7-13-56 \\
8-14-56\end{array}$ & $\begin{array}{l}0 \\
0 \\
0 \\
21.80 \\
0 \\
0 \\
0 \\
0 \\
0\end{array}$ & $\begin{array}{r}8-27-56 \\
11-13-56 \\
12-31-56 \\
2-5-57 \\
3-6-57 \\
7-30-57 \\
9-4-57 \\
12-2-57\end{array}$ & $\begin{array}{l}0 \\
0 \\
0 \\
0 \\
1.87 \\
2.48 \\
.05 \\
7.16\end{array}$ & $\begin{array}{r}1-27-58 \\
2-18-58 \\
3-31-58 \\
5-12-58 \\
7-29-58 \\
9-17-58 \\
10-28-58 \\
11-17-58\end{array}$ & $\begin{array}{r}9.80 \\
7.02 \\
24.4 \\
23.2 \\
.92 \\
.59 \\
2.42\end{array}$ & $\begin{array}{r}2-16-59 \\
3-31-59 \\
6-15-59 \\
8-13-59 \\
11-8-59 \\
1-26-60 \\
3-8-60 \\
9-20-60\end{array}$ & $\begin{array}{c}1.28 \\
1.91 \\
.32 \\
14.3 \\
1.80 \\
28.6 \\
1.86\end{array}$ \\
\hline
\end{tabular}

Salt Creek near Yale, Oklahoma, Miscellaneous site.

Lat. $36^{\circ} 09^{\prime} 14^{\prime \prime}$, 1 ong. $96^{\circ} 47^{\prime} 25^{\prime \prime}$, west line sec.4, T.19 N., R.5 E., at bridge on State Highway 18 , 5 miles northwest of Yale, Oklahoma.

Date $\begin{gathered}\text { Discharge } \\ \left(\mathrm{ft}^{3} / \mathrm{s}\right)\end{gathered}$

$10-25-48 \quad .01$

Salt Creek near Shidler, Oklahoma, 07152400 , partial record site.

Lat. $36^{\circ} 45^{\prime} 00^{\prime \prime}$, long. $96^{\circ} 40^{\prime} 00^{\prime \prime}, \mathrm{NW} 1 / 4 \mathrm{NW} 1 / 4 \mathrm{sec} .10$, T.26 N., R.6 E., at bridge on State Highway 18, 2 miles southeast of Shidler, Okla.

\begin{tabular}{|c|c|c|c|c|c|c|c|}
\hline Date & $\begin{array}{c}\text { Discharge } \\
\left(\mathrm{ft}^{3} / \mathrm{s}\right)\end{array}$ & Date & $\begin{array}{c}\text { Discharge } \\
\left(\mathrm{ft}^{3} / \mathrm{s}\right)\end{array}$ & Date & $\begin{array}{c}\text { Discharge } \\
\left(\mathrm{ft}^{3} / \mathrm{s}\right)\end{array}$ & Date & $\begin{array}{c}\text { Discharge } \\
\left(\mathrm{ft}^{3} / \mathrm{s}\right)\end{array}$ \\
\hline $\begin{array}{r}11-10-53 \\
12-16-53 \\
1-12-54 \\
2-16-54 \\
3-1-54 \\
4-5-54 \\
4-26-54 \\
5-11-54 \\
6-2-54 \\
7-6-54 \\
8-2-54 \\
9-9-54 \\
10-13-54 \\
11-5-54 \\
12-15-54 \\
1-3-55 \\
2-1-55 \\
3-7-55 \\
4-5-55\end{array}$ & $\begin{array}{l}0 \\
0 \\
0 \\
0 \\
0 \\
0 \\
0 \\
5.93 \\
.76 \\
0 \\
0 \\
0 \\
0 \\
0 \\
0 \\
0 \\
0 \\
0 \\
0\end{array}$ & $\begin{array}{r}5-3-55 \\
6-7-55 \\
7-13-55 \\
8-1-55 \\
11-5-57 \\
12-3-57 \\
1-3-58 \\
2-5-58 \\
3-3-58 \\
5-20-58 \\
6-10-58 \\
8-6-58 \\
10-9-58 \\
11-18-58 \\
1-29-59 \\
3-10-59 \\
4-1-59 \\
6-9-59\end{array}$ & $\begin{array}{l}0 \\
9.96 \\
0 \\
0 \\
0 \\
0 \\
0 \\
2.17 \\
1.18 \\
6.94 \\
.78 \\
4.43 \\
0 \\
0 \\
0 \\
17.4 \\
28.2 \\
9.88\end{array}$ & $\begin{array}{r}8-18-59 \\
1-28-59 \\
11-19-50 \\
12-15-59 \\
5-18-60 \\
6-14-60 \\
8-17-60 \\
9-20-60 \\
10-25-60 \\
1-17-61 \\
2-15-61 \\
3-16-61 \\
4-18-61 \\
6-15-61 \\
8-2-61 \\
10-3-61 \\
11-14-61 \\
12-19-61\end{array}$ & $\begin{array}{c}14.3 \\
27.0 \\
22.1 \\
11.9 \\
17.3 \\
9.05 \\
0 \\
0 \\
0 \\
3.93 \\
3.56 \\
10.2 \\
28.5 \\
63.1 \\
2.99 \\
31.7 \\
148 . \\
174 .\end{array}$ & $\begin{array}{r}2-13-62 \\
5-16-62 \\
8-14-62 \\
10-16-62 \\
12-19-62 \\
3-19-63 \\
4-16-63 \\
6-18-63 \\
8-21-63 \\
12-17-63 \\
3-17-64 \\
5-5-64 \\
6-5-64 \\
3-9-65 \\
7-15-65 \\
11-3-65 \\
3-9-66 \\
6-1-66\end{array}$ & $\begin{array}{c}23.3 \\
2.19 \\
.75 \\
0 \\
0 \\
19.0 \\
3.96 \\
0 \\
0 \\
0 \\
0 \\
0 \\
4.54 \\
22.4 \\
10.1 \\
0 \\
9.3 \\
2.6\end{array}$ \\
\hline
\end{tabular}


Salt Fork Arkansas River near Alva, Oklahoma, 07148400,partial record site.

Lat. $36^{\circ} 48^{\prime} 45^{\prime \prime}$, long. $98^{\circ} 38^{\prime} 45^{\prime \prime}, \mathrm{Sh}^{\prime} 1 / 4 \mathrm{SW}^{\prime} 1 / 4 \mathrm{sec} .18$, T.27 N., R.13 h, at bridge on State Highway 14,1 mile northeast of Alva, Okla.

\begin{tabular}{|c|c|c|c|c|c|c|c|}
\hline Date & $\begin{array}{c}\text { Discharge } \\
\left(\mathrm{ft}^{3} / \mathrm{s}\right)\end{array}$ & Date & $\begin{array}{c}\text { Discharge } \\
\left(\mathrm{ft}^{3} / \mathrm{s}\right)\end{array}$ & Date & $\begin{array}{c}\text { Discharge } \\
\left(\mathrm{ft}^{3} / \mathrm{s}\right)\end{array}$ & Date & $\begin{array}{c}\text { Discharge } \\
\left(\mathrm{ft}^{3} / \mathrm{s}\right)\end{array}$ \\
\hline $\begin{array}{r}11-5-52 \\
12-2-52 \\
1-6-53 \\
2-3-53 \\
3-2-53\end{array}$ & $\begin{array}{r}.19 \\
.67 \\
44.0 \\
19.5 \\
96.0\end{array}$ & $\begin{array}{l}4-28-53 \\
6-1-53 \\
6-20-53 \\
8-5-53\end{array}$ & $\begin{array}{r}5.45 \\
.95 \\
.56 \\
2.06\end{array}$ & $\begin{array}{r}0-1-53 \\
10-1-53 \\
11-2-53 \\
1-5-54\end{array}$ & $\begin{array}{r}.26 \\
.55 \\
1.08 \\
10.4\end{array}$ & $\begin{array}{l}2-1-54 \\
3-1-54 \\
4-27-54 \\
6-2-54\end{array}$ & $\begin{array}{l}38.0 \\
20.2 \\
15.3 \\
42.7\end{array}$ \\
\hline
\end{tabular}

Salt Fork Arkansas River near Tonkawa, Oklahoma, Miscellaneous site.

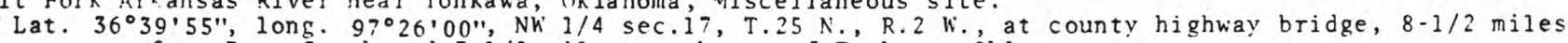
upstream from Deer Creek and 7-i/2 miles southwest of Tonkawa, ikla.

$$
\text { Date } \begin{gathered}
\text { Discharge } \\
\left(\mathrm{ft}^{3} / \mathrm{s}\right)
\end{gathered}
$$

$10-21-52$

$$
27.7
$$

Salt Fork Red River near Gould, Nklahoma, Miscellaneous site.

Lat. $34^{\circ} 51^{\prime} 28^{\prime \prime}$, long. $99^{\circ} 43^{\prime} 25^{\prime \prime}, \mathrm{SE} 1 / 4 \mathrm{SE} 1 / 4 \mathrm{sec} .6, \mathrm{~T} .4 \mathrm{~N} ., \mathrm{R} .24 \mathrm{~W}$, at site 12 miles north of Gould, Okla.

$$
\begin{array}{cc}
\text { Date } & \begin{array}{c}
\text { Discharge } \\
\left(\mathrm{ft}^{3} / \mathrm{s}\right)
\end{array} \\
2-10-54 & 17.9
\end{array}
$$

\begin{tabular}{|c|c|c|c|c|c|c|c|}
\hline Date & $\begin{array}{c}\text { Discharge } \\
\left(\mathrm{ft}^{3} / \mathrm{s}\right)\end{array}$ & Date & $\begin{array}{c}\text { Discharge } \\
\left(\mathrm{ft}^{3} / \mathrm{s}\right)\end{array}$ & Date & $\begin{array}{c}\text { Discharge } \\
\left(\mathrm{ft}^{3} / \mathrm{s}\right)\end{array}$ & Date & $\begin{array}{c}\text { Discharge } \\
\left(\mathrm{ft}^{3} / \mathrm{s}\right)\end{array}$ \\
\hline $\begin{array}{l}3-29-54 \\
3-29-54\end{array}$ & $\begin{array}{l}6.36 \\
6.58\end{array}$ & $3-29-54$ & 5.76 & $3-29-54$ & 4.98 & $\begin{array}{l}3-20-54 \\
3-29-54\end{array}$ & $\begin{array}{l}4.47 \\
4.50\end{array}$ \\
\hline
\end{tabular}

Salt Fork Red River near Mangum, Oklahoma, Miscellaneous site.

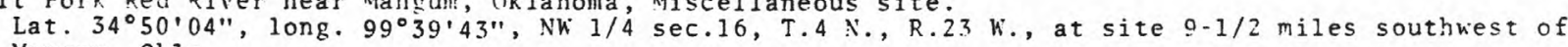
Mangum, Okla.

Salt Fork Red River near Reed, Nklahoma, Miscellaneous site.

Lat. $34^{\circ} 52^{\prime} 09^{\prime \prime}$, long. $99^{\circ} 42^{\prime} 17^{\prime \prime}$ SE $1 / 4 \mathrm{SE} 1 / 4 \mathrm{sec} .35$, T.5 N., R. 24 W., at county highway bridge, 3 miles south of Reed, Okla.

$$
\begin{array}{cc}
\text { Date } & \begin{array}{c}
\text { Discharge } \\
\left(\mathrm{ft}^{3} / \mathrm{s}\right)
\end{array} \\
2-10-54 & 17.9
\end{array}
$$

Salt Fori Red River near Russell, Oklahoma, Miscellaneous site.

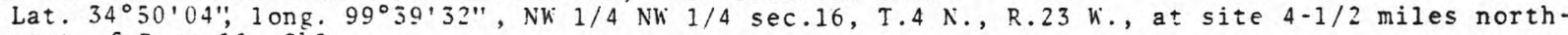
east of Russe11, okia.

$$
\begin{array}{cc}
\text { Date } & \begin{array}{c}
\text { Discharge } \\
\left(\mathrm{ft}^{3} / \mathrm{s}\right)
\end{array} \\
2-10-54 & 18.4
\end{array}
$$


LOW-FLOW MEASUREMENTS--Cont inued.

Salt Fork Red River near Vinson, Oklahoma, Miscellaneous site.

Lat. $34^{\circ} 50^{\prime} 45^{\prime \prime}, 1$ ong. $99^{\circ} 54^{\prime} 30^{\prime \prime}, \mathrm{SE} 1 / 4 \mathrm{sec} .3, \mathrm{~T} .4 \mathrm{~N} ., \mathrm{R} .26 \mathrm{~W}$, at bridge on State Highway 30 , 5 miles southwest of Vinson, 0kla.

$\begin{array}{ccccccc}\text { Date } & \begin{array}{c}\text { Discharge } \\ \left(\mathrm{ft}^{3} / \mathrm{s}\right)\end{array} & \text { Date } & \begin{array}{c}\text { Discharge } \\ \left(\mathrm{ft}^{3} / \mathrm{s}\right)\end{array} & \text { Date } & \begin{array}{c}\text { Discharge } \\ \left(\mathrm{ft}^{3} / \mathrm{s}^{2}\right)\end{array} & \begin{array}{c}\text { Date } \\ \left(\mathrm{ft}^{3} / \mathrm{s}\right)\end{array} \\ 3-11-53 & 2.97 & 11-16-53 & 9.30 & 2-10-54 & 18.1 & 12-23-54 \\ 9-29-53 & 0 & 12-21-53 & 20.2 & 10-19-54 & 0 & 0\end{array}$

Sand Creek near Okesa, Oklahoma, Miscellaneous site.

Lat. $36^{\circ} .43^{\prime} 00^{\prime \prime}$, long. $96^{\circ} 08^{\prime} 00^{\prime \prime}$, NE $1 / 4 \mathrm{sec} .20$, T.26 N., R.11 E., l/4 mile upstream from county highway bridge and $1 / 2$ mile north of Okesa, Okla.

\begin{tabular}{|c|c|c|c|c|c|c|c|}
\hline Date & $\begin{array}{c}\text { Discharge } \\
\left(\mathrm{ft}^{3} / \mathrm{s}\right)\end{array}$ & Date & $\begin{array}{c}\text { Discharge } \\
\left(\mathrm{ft}^{3} / \mathrm{s}\right)\end{array}$ & Date & $\begin{array}{c}\text { Discharge } \\
\left(\mathrm{ft}^{3} / \mathrm{s}\right)\end{array}$ & Date & $\begin{array}{c}\text { Discharge } \\
\left(f t^{3} / \mathrm{s}\right)\end{array}$ \\
\hline $\begin{array}{r}10-31-51 \\
12-12-51 \\
3-4-52 \\
4-22-52 \\
6-3-52 \\
7-21-53 \\
9-2-52 \\
1-6-53 \\
2-17-53 \\
3-23-53\end{array}$ & $\begin{array}{r}109.0 \\
20.0 \\
350.0 \\
291.0 \\
5.03 \\
.19 \\
0 \\
0 \\
.03 \\
1.58\end{array}$ & $\begin{array}{r}5-5-53 \\
6-1-53 \\
6-22-53 \\
8-13-53 \\
9-21-53 \\
10-21-53 \\
11-10-53 \\
12-16-53 \\
1-11-54 \\
2-15-54\end{array}$ & $\begin{aligned} 11.5 \\
2.01 \\
.46 \\
0 \\
0 \\
.01 \\
.01 \\
.69 \\
.16 \\
.34\end{aligned}$ & $\begin{array}{r}3-15-54 \\
4-5-54 \\
4-26-54 \\
5-10-54 \\
6-2-54 \\
7-6-54 \\
8-2-54 \\
9-9-54 \\
10-13-54 \\
11-3-54\end{array}$ & $\begin{array}{l}.09 \\
.02 \\
.05 \\
13.6 \\
23.1 \\
0.05 \\
0 \\
0 \\
3.24\end{array}$ & $\begin{array}{r}12-14-54 \\
1-3-55 \\
2-1-55 \\
3-2-55 \\
4-4-55 \\
5-2-55 \\
6-7-55 \\
7-13-55 \\
8-1-55 \\
9-2-55\end{array}$ & $\begin{array}{c}.23 \\
7.58 \\
2.31 \\
3.64 \\
24.8 \\
4.03 \\
14.9 \\
.10 \\
0 \\
0\end{array}$ \\
\hline
\end{tabular}

Sand Creek near Roll, Oklahoma, Miscellaneous site.

Lat. $35^{\circ} 48^{\prime} 48^{\prime \prime}$, long. $99^{\circ} 49^{\prime} 43^{\prime \prime}$, NW $1 / 4 \mathrm{sec} .14, \mathrm{~T} .15 \mathrm{~N} ., \mathrm{R} .25 \mathrm{~W}$. , at county highway bridge, 7 miles west of Rol1, Okla.

$\begin{array}{rr}\text { Date } & \begin{array}{r}\text { Discharge } \\ \left(\mathrm{ft}^{3} / \mathrm{s}\right)\end{array} \\ 9-13-55 & .16\end{array}$

Sand Creek near Sharon, Oklahoma, Miscellaneous site.

Lat. $36^{\circ} 19^{\prime} 37^{\prime \prime}$, long. $99^{\circ} 13^{\prime} 56^{\prime \prime}$, SW $1 / 4 \mathrm{SW} 1 / 4 \mathrm{sec} .4, \mathrm{~T} .21 \mathrm{~N}$. , R.19 w., 6 miles northeast of Sharon, Okla.

$\begin{array}{lrrr}\text { Date } & \begin{array}{c}\text { Discharge } \\ \left(\mathrm{ft}^{3} / \mathrm{s}\right)\end{array} & \text { Date } & \begin{array}{r}\text { Discharge } \\ \left(\mathrm{ft}^{3} / \mathrm{s}\right)\end{array} \\ 2-20-44 & .41 & 9-28-48 & .40\end{array}$

Sand Creel near Woodward, Oklahoma, Miscellaneous site.

Lat. $36^{\circ} 19^{\prime} 37^{\prime \prime}$, long. $99^{\circ} 13^{\prime} 56^{\prime \prime}$, SW $1 / 4 \mathrm{sec} .4, \mathrm{~T} .21$ N., R.19 W., at bridge on U.S. Highway 270 , 11 miles southeast of woodward, Okla.
Date
Discharge
$6-5-56$
1. 51 
Sandy Creek near Byron, Oklahoma, 07149700 , partial record site.

Lat. $36^{\circ} 53^{\prime} 03^{\prime \prime}$, long. $98^{\circ} 13^{\prime} 02^{\prime \prime}$, NE $1 / 4 \mathrm{NE} 1 / 4 \mathrm{sec} .25, \mathrm{~T} .28 \mathrm{~N} ., \mathrm{R} .10 \mathrm{~W}$, at county road bridge a quarter of a mile downstream from Little Sandy Creek, 4-1/2 miles southeast of Byron and about 6 miles upstream from Great Salt Plains Reservoir.

\begin{tabular}{|c|c|c|c|c|c|c|c|}
\hline Date & $\begin{array}{c}\text { Discharge } \\
\left(\mathrm{ft}^{3} / \mathrm{s}\right)\end{array}$ & Date & $\begin{array}{c}\text { Discharge } \\
\left(\mathrm{ft}^{3} / \mathrm{s}\right)\end{array}$ & Date & $\begin{array}{c}\text { Di scharge } \\
\left(\mathrm{ft}^{3} / \mathrm{s}\right)\end{array}$ & Date & $\begin{array}{c}\text { Discharge } \\
\left(\mathrm{ft}^{3} / \mathrm{s}\right)\end{array}$ \\
\hline $\begin{array}{r}10-18-64 \\
1-19-65 \\
8-17-65 \\
12-7-65 \\
3-10-66 \\
7-8-66 \\
9-9-66 \\
11-22-66\end{array}$ & $\begin{array}{c}1.15 \\
23.5 \\
5.87 \\
12.0 \\
25.0 \\
1.1 \\
1.6 \\
.94\end{array}$ & $\begin{array}{r}1-31-67 \\
8-12-67 \\
1-29-68 \\
6-6-68 \\
7-17-68 \\
11-25-68 \\
4-16-69 \\
7-10-69\end{array}$ & $\begin{array}{l}1.9 \\
3.0 \\
4.0 \\
2.8 \\
.19 \\
3.9 \\
9.1 \\
5.2\end{array}$ & $\begin{array}{r}12-18-69 \\
2-3-70 \\
5-19-70 \\
7-7-70 \\
11-10-70 \\
3-23-71 \\
5-5-71 \\
8-18-71\end{array}$ & $\begin{array}{r}9.0 \\
8.7 \\
9.3 \\
11.0 \\
6.0 \\
8.7 \\
6.6 \\
8.7\end{array}$ & $\begin{array}{r}11-17-71 \\
3-9-72 \\
4-19-72 \\
8-2-72 \\
10-25-72 \\
2-28-73 \\
5-23-73\end{array}$ & $\begin{array}{l}4.0 \\
3.3 \\
2.4 \\
2.2 \\
19 . \\
38 . \\
55 .\end{array}$ \\
\hline
\end{tabular}

Sandstone Creek near Cheyenne, Oklahoma, Miscellaneous site.

Lat. $35^{\circ} 36^{\circ} 28^{\prime \prime}$, long. $99^{\circ} 33^{\prime} 25^{\prime \prime}$, NE $1 / 4$ sec. 23 , T.13 N., R. $22 \mathrm{~W}$, at county highway bridge, $1 / 2$

mile above mouth and 9 miles east of Cheyenne, Okla.
Date Discharge
$\left(\mathrm{ft}^{3} / \mathrm{s}\right)$
$1-5-56 \quad 3.48$

Sergeant Major Creek near Cheyenne, Oklahoma, Miscellaneous site.

Lat. $35^{\circ} 33^{\prime} 28^{\prime \prime}$, long. $99^{\circ} 43^{\prime} 08^{\prime \prime}$, NE $1 / 4 \mathrm{SE} 1 / 4 \mathrm{sec} .35$, T.13 N., R.24 W., at site $4-1 / 2$ miles southwest of Cheyenne, okla.

$\begin{array}{cccccr}\text { Date } & \begin{array}{c}\text { Discharge } \\ \left(\mathrm{ft}^{3} / \mathrm{s}\right)\end{array} & \text { Date } & \begin{array}{c}\text { Discharge } \\ \left(\mathrm{ft}^{3} / \mathrm{s}\right)\end{array} & \text { Date } & \begin{array}{r}\text { Nischarge } \\ \left(\mathrm{ft}^{3} / \mathrm{s}\right)\end{array} \\ 2-5-54 & 1.14 & 7-30-56 & .41 & 9-13-56 & .35\end{array}$

Sheep Creek near Harden City, Oklahoma, Miscellaneous site.

Lat. $34^{\circ} 36^{\prime} 00^{\prime \prime}$, long. $96^{\circ} 32^{\prime} 27^{\prime \prime}$, in SW $1 / 4 \mathrm{NW} 1 / 4$ sec. 34 , T. 2 N., R.7 E., at county highway bridge 1-1/4 miles above mouth and 2 miles east of Harden City, Oklahoma.

Date Discharge

$12-12-57 \quad 11.6$

Shell Creek near Sand Springs, Oklahoma, Miscellaneous site.

Lat. $36^{\circ} 09^{\prime} 14^{\prime \prime}$, long. $96^{\circ} 16^{\prime} 20^{\prime \prime}$, SW $1 / 4 \mathrm{sec} .5, \mathrm{~T} .19 \mathrm{~N} ., \mathrm{R} .10 \mathrm{E}$. , at bridge on county road at wekiwa, $2-3 / 4$ miles west of Sand Springs, Okla.

Discharge
$\left(\mathrm{ft}^{3} / \mathrm{s}\right)$

Skeleton Creek near Lovell, nklahoma, Miscellaneous site.

Lat. $36^{\circ} 04^{\prime} 37^{\prime \prime}$, long. $97^{\circ} 36^{\prime} 34^{\prime \prime}$, west line sec.34, T.ig N., R.4 K., at bridge on old State Highway 74,2 miles north of Lovell, Oklahoma.

$\begin{array}{crrr}\text { Date } & \begin{array}{r}\text { Discharge } \\ \left(\mathrm{ft}^{3} / \mathrm{s}\right)\end{array} & \text { Date } & \begin{array}{r}\text { Discharge } \\ \left(\mathrm{ft}^{3} / \mathrm{s}\right)\end{array} \\ 10-27-48 & .94 & 7-27-49 & 3.82\end{array}$


Skull Creek near Norfold, Oklahoma, Miscellaneous site.

Lat. $36^{\circ} 03^{\prime} 08^{\prime \prime}$, long. $96^{\circ} 38^{\prime} 24^{\prime \prime}$, NE $1 / 4$ secl3, T.18 N., R.6 E., at bridge on county road, $2-1 / 4$ miles south of Norfolk, Okla.

$\begin{array}{cr}\text { Date } & \begin{array}{c}\text { Discharge } \\ \left(\mathrm{ft}^{3} / \mathrm{s}\right)\end{array} \\ 10-23-52 & 1.14\end{array}$

Snake Creek near Bixby, Oklahoma, 07165550 , partial record site.

Lat. $35^{\circ} 49^{\prime} 08^{\prime \prime}$, long. $95^{\circ} 53^{\prime} 18^{\prime \prime}$, in NW $1 / 4 \mathrm{SW} 1 / 4 \mathrm{sec} .36, \mathrm{~T} .16 \mathrm{~N}$. , R.13 E., Okmulgee County, on right bank 5.5 miles upstream from Duck Creek, 8.8 miles south of Bixby, and at mile 11.0 .

\begin{tabular}{|c|c|c|c|c|c|c|c|}
\hline Date & $\begin{array}{c}\text { Discharge } \\
\left(\mathrm{ft}^{3} / \mathrm{s}\right)\end{array}$ & Date & $\begin{array}{c}\text { Discharge } \\
\left(f t^{3} / s\right)\end{array}$ & Date & $\begin{array}{c}\text { Discharge } \\
\left(\mathrm{ft}^{3} / \mathrm{s}\right)\end{array}$ & Date & $\begin{array}{c}\text { Discharge } \\
\left(\mathrm{ft}^{3} / \mathrm{s}\right)\end{array}$ \\
\hline $\begin{array}{l}2-17-71 \\
5-21-71\end{array}$ & $\begin{array}{l}2.2 \\
.04\end{array}$ & $\begin{array}{l}7-30-71 \\
9-2-71\end{array}$ & $\begin{array}{l}0 \\
0\end{array}$ & $\begin{array}{l}4-3-72 \\
6-7-72\end{array}$ & $l_{0}^{1.6}$ & $8-9-72$ & 0 \\
\hline
\end{tabular}

Soldier Creek near Piedmont, Oklahoma, Miscellaneous site.

Lat. $35^{\circ} 38^{\prime} 32^{\prime \prime}$, long. $97^{\circ} 40^{\prime} 00^{\prime \prime}$, east line NE $1 / 4 \mathrm{sec} 31, \mathrm{~T} .14 \mathrm{~N} ., \mathrm{R} .4 \mathrm{~W} .$, at county road bridge 5 miles east of Piedmont.

Date Discharge
$\left(\mathrm{ft}^{3} / \mathrm{s}\right)$

$10-29-59 \quad 1.50$

Spring Creek near Ada, Oklahoma, Miscellaneous site.

Lat. $34^{\circ} 47^{\prime} 00^{\prime \prime}$, long. $96^{\circ} 45^{\prime} 00^{\prime \prime}$, SW $1 / 4$ sec. 26, T. 4 N., R. 5 E., at county highway bridge, 4 miles west of city limits, Ada, Okla.

$\begin{array}{ccccccr}\text { Date } & \begin{array}{c}\text { Discharge } \\ \left(\mathrm{ft}^{3} / \mathrm{s}\right)\end{array} & \text { Date } & \begin{array}{c}\text { Discharge } \\ \left(\mathrm{ft}^{3} / \mathrm{s}\right)\end{array} & \text { Date } & \begin{array}{c}\text { Discharge } \\ \left(\mathrm{ft}^{3} / \mathrm{s}^{3}\right)\end{array} & \begin{array}{c}\text { Date } \\ \left(\mathrm{ft}^{3} / \mathrm{s}^{3}\right)\end{array} \\ 9-7-51 & 0 & 12-11-51 & 0 & 4-21-52 & 81.6 & 9-2-52 \\ 10-2-51 & 0 & 1-14-52 & 0 & 6-2-52 & 42.8 & 10-6-5.3 \\ 10-31-51 & 0 & 2-26-52 & 2.50 & 7-22-52 & 0 & 0\end{array}$

Spring Creek near Eakly, Oklahoma, Miscellaneous site.

Lat. $35^{\circ} 16^{\prime} 09^{\prime \prime}$, long. $98^{\circ} 32^{\prime} 05^{\prime \prime}$, NE $1 / 4 \mathrm{sec} .24, \mathrm{~T} .9 \mathrm{~N} ., \mathrm{R} .13 \mathrm{~K}$, at bridge on State Highway 41 , 2 miles southeast of Eakley, Oklahoma.

$\begin{array}{crcr}\text { Date } & \begin{array}{c}\text { Discharge } \\ \left(\mathrm{ft}^{3} / \mathrm{s}\right)\end{array} & \text { Date } & \begin{array}{r}\text { Discharge } \\ \left(\mathrm{ft}^{3} / \mathrm{s}\right)\end{array} \\ 10-23-46 & 3.48 & 10-23-46 & 3.64\end{array}$

Spring Creek near Locust Grove, Nklahoma, Miscellaneous site.

Lat. $36^{\circ} 09^{\prime} 28^{\prime \prime}$, long. $95^{\circ} 09^{\prime} 43^{\prime \prime}$, SE $1 / 4 \mathrm{sec} .3, \mathrm{~T} .19 \mathrm{~N} ., \mathrm{R} .20 \mathrm{E}$, at bridge on State Highway 82 , 3.5 miles south of Locust Grove, Okla.

$\begin{array}{cc}\text { Date } & \begin{array}{c}\text { Discharge } \\ \left(\mathrm{ft}^{3} / \mathrm{s}\right)\end{array} \\ 7-12-60 & 19.2\end{array}$


Spring Creek near Sayre, Oklahoma, Miscellaneous site.

Lat. $35^{\circ} 11^{\prime} 04^{\prime \prime}$, long. $99^{\circ} 36^{\prime} 34^{\prime \prime}$, NE $1 / 4$ sec.14, T.8 N., R.23 W., at site $7-1 / 2$ miles southeast of Sayre, Okla.

$\begin{array}{rr}\text { Date } & \begin{array}{r}\text { Discharge } \\ \left(\mathrm{ft}^{3} / \mathrm{s}\right)\end{array} \\ 8-21-52 & 1.41\end{array}$

Spring Creek near Swan Lake, Oklahoma, Miscellaneous site.

Lat. $35^{\circ} 16^{\prime} 23^{\prime \prime}$, long. $98^{\circ} 36^{\circ} 34^{\prime \prime}$, SE $1 / 4 \mathrm{sec} .22$, T.9 N., R.13 W., at county highway bridge, 2 miles west of Swan Lake, Okla.

$\begin{array}{cc}\text { Date } & \begin{array}{c}\text { Discharge } \\ \left(\mathrm{ft}^{3} / \mathrm{s}\right)\end{array} \\ 9-15-52 & 0\end{array}$

Spring Lake Creek near Cordell, Oklahoma, Miscellaneous site.

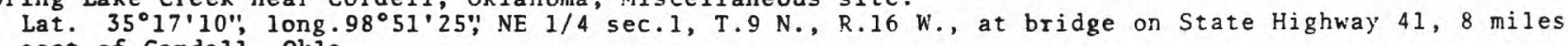
east of Cordeli, Ok1a.

$\begin{array}{cr}\text { Date } & \begin{array}{r}\text { Discharge } \\ \left(\mathrm{ft}^{3} / \mathrm{s}\right)\end{array} \\ 10-10-52 & .46\end{array}$

Spring No. 1 near Sayre, Oklahoma, Miscellaneous site.

Lat. $35^{\circ} 11^{\prime} 04^{\prime \prime}$, long. $99^{\circ} 36^{\prime} 34^{\prime \prime}$, SW $1 / 4 \mathrm{NE} 1 / 4 \mathrm{sec} .10$, T.8 N., R.23 W., at site $7-1 / 2 \mathrm{miles}$ southeast of Sayre, Ok1a:

$\begin{array}{crrr}\text { Date } & \begin{array}{r}\text { Discharge } \\ \left(\mathrm{ft}^{3} / \mathrm{s}\right)\end{array} & \text { Date } & \begin{array}{r}\text { Discharge } \\ \left(\mathrm{ft}^{3} / \mathrm{s}\right)\end{array} \\ 10-8-52 & 1.21 & 4-1-53 & 1.28\end{array}$

Starvation Creek near Prentiss, Ok1ahoma, 07301452, partial record site.

Lat. $35^{\circ} 19^{\prime} 25^{\prime \prime}$, long. $99^{\circ} 45^{\prime} 30^{\prime \prime}$, in Sh $1 / 4 \mathrm{SE} 1 / 4$ sec.20, T.10 N., R. $24 \mathrm{~K}$., Beckham County, at bridge on State Highway 152, 3 miles southeast of Prentiss, and about 2 miles upstream from mouth.

\begin{tabular}{|c|c|c|c|c|c|c|c|}
\hline Date & $\begin{array}{c}\text { Discharge } \\
\left(\mathrm{ft}^{3} / \mathrm{s}\right)\end{array}$ & Date & $\begin{array}{c}\text { Discharge } \\
\left(\mathrm{ft}^{3} / \mathrm{s}\right)\end{array}$ & Date & $\begin{array}{c}\text { Tischarge } \\
\left(\mathrm{ft}^{3} / \mathrm{s}\right)\end{array}$ & Date & $\begin{array}{c}\text { Discharge } \\
\left(\mathrm{ft}^{3} / \mathrm{s}\right)\end{array}$ \\
\hline $\begin{array}{r}10-27-64 \\
3-8-65 \\
7-9-65 \\
11-9-65 \\
2-7-66 \\
5-9-66 \\
11-22-66 \\
2-16-67\end{array}$ & $\begin{array}{l}.30 \\
1.05 \\
1.80 \\
3.60 \\
2.10 \\
1.9 \\
1.6 \\
1.1\end{array}$ & $\begin{array}{r}5-23-67 \\
9-1-67 \\
12-13-67 \\
5-28-68 \\
6-26-68 \\
9-16-68 \\
12-11-68 \\
4-4-69\end{array}$ & $\begin{array}{l}.41 \\
1.3 \\
.52 \\
.56 \\
.65 \\
.60 \\
3.9 \\
4.3\end{array}$ & $\begin{array}{r}6-11-69 \\
12-16-69 \\
2-17-70 \\
6-11-70 \\
8-12-70 \\
11-24-70 \\
1-27-71 \\
5-19-71\end{array}$ & $\begin{array}{c}4.1 \\
.92 \\
1.3 \\
.63 \\
.18 \\
.12 \\
1.3 \\
.66\end{array}$ & $\begin{array}{r}8-12-71 \\
10-14-71 \\
3-8-72 \\
4-18-72 \\
11-14-72 \\
2-7-73 \\
5-30-73\end{array}$ & $\begin{array}{l}.19 \\
.25 \\
.53 \\
.41 \\
.44 \\
.60 \\
1.10\end{array}$ \\
\hline
\end{tabular}

Stillwater Creek near Ripley, Oklahoma, Miscellaneous site. Lat. $36^{\circ} 03^{\prime} 41^{\prime \prime}$, long. $96^{\circ} 56^{\circ} 04^{\prime \prime}$, SE $1 / 4 \mathrm{sec} .11$, T.18 N., R. $3 \mathrm{E}$, at bridge on county road, $1 / 8 \mathrm{mile}$ west of Mehan and 3 miles northwest of Ripley, Okia.

$\begin{array}{cr}\text { Date } & \begin{array}{r}\text { Discharge } \\ \left(\mathrm{ft}^{3} / \mathrm{s}\right)\end{array} \\ 10-23-52 & 3.31\end{array}$


Stinking Creek near Carnegie, Oklahoma, 07325400 , partial record site.

Lat. $35^{\circ} 05^{\prime} 00^{\prime \prime}$, long. $98^{\circ} 35^{\prime} 00^{\prime \prime}$, center sec.11, T.7 N., R.14 W., at bridge on State Highway 9 , 2-1/4 miles southwest of Carnegie, Okia.

\begin{tabular}{|c|c|c|c|c|c|c|c|}
\hline Date & $\begin{array}{c}\text { Discharge } \\
\left(\mathrm{ft}^{3} / \mathrm{s}\right)\end{array}$ & Date & $\begin{array}{c}\text { Discharge } \\
\left(\mathrm{ft}^{3} / \mathrm{s}\right)\end{array}$ & Date & $\begin{array}{c}\text { Discharge } \\
\left(\mathrm{ft}^{3} / \mathrm{s}\right)\end{array}$ & Date & $\begin{array}{c}\text { Discharge } \\
\left(\mathrm{ft}^{3} / \mathrm{s}\right)\end{array}$ \\
\hline $\begin{array}{r}9-5-51 \\
11-29-51 \\
1-15-52 \\
3-5-52 \\
4-22-52 \\
5-16-52 \\
6-4-52 \\
7-9-52 \\
8-20-52 \\
10-7-52 \\
11-4-52 \\
12-1-52\end{array}$ & $\begin{array}{l}0 \\
.74 \\
.35 \\
1.06 \\
8.70 \\
0 \\
2.93 \\
0 \\
0 \\
0 \\
0 \\
0\end{array}$ & $\begin{array}{r}12-11-52 \\
1-20-53 \\
3-2-53 \\
3-31-53 \\
5-18-53 \\
6-22-53 \\
7-28-53 \\
8-25-53 \\
10-1-53 \\
11-3-53 \\
1-19-54 \\
2-15-54\end{array}$ & $\begin{array}{l}0 \\
0 \\
0 \\
0 \\
1.75 \\
0 \\
1.33 \\
18.3 \\
0 \\
.55 \\
.04 \\
.04\end{array}$ & $\begin{array}{r}3-16-54 \\
5-5-54 \\
6-3-54 \\
6-29-54 \\
8-2-54 \\
9-7-54 \\
10-19-54 \\
11-15-54 \\
12-16-54 \\
1-17-55 \\
2-14-55 \\
3-16-55\end{array}$ & $\begin{array}{l}.04 \\
3.88 \\
3.79 \\
0 \\
0 \\
0 \\
0 \\
0 \\
0 \\
0 \\
0 \\
0\end{array}$ & $\begin{array}{r}6-15-55 \\
7-20-55 \\
8-22-55 \\
2-4-58 \\
2-24-59 \\
8-21-59 \\
9-15-59 \\
5-9-60 \\
11-30-60 \\
3-7-61 \\
3-15-61 \\
6-2-61\end{array}$ & $\begin{array}{r}8.99 \\
.64 \\
0 \\
9.33 \\
.90 \\
.97 \\
0 \\
12.60 \\
4.14 \\
6.94 \\
5.82 \\
.05\end{array}$ \\
\hline
\end{tabular}

Stinking Creek near Humphreys, Ok lahoma, Miscellaneous site.

Lat. $34^{\circ} 33^{\prime} 14^{\prime \prime}$, long. $99^{\circ} 12^{\prime} 37^{\prime \prime}$, NW $1 / 4 \mathrm{sec} .28$, T.1 N., R.19 W., at bridge on unimpooved county road, $2-1 / 2$ miles southeast of Humphreys, Okla.
Date
Discharge
$\left(\mathrm{ft}^{3} / \mathrm{s}\right)$
$4-22-53$
.72

Sugar Creek near Anadarko, Oklahoma, Miscellaneous site.

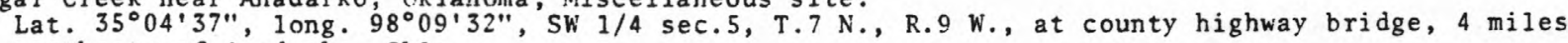
northeast of Anadarko, Okla.

$\begin{array}{cr}\text { Date } & \begin{array}{r}\text { Discharge } \\ \left(\mathrm{ft}^{3} / \mathrm{s}\right)\end{array} \\ 7-10-52 & .08\end{array}$

Sugar Creek near Gracemont, Oklahoma, 07327000 , partial record site.

Lat. $35^{\circ} 11^{\prime} 18^{\prime \prime}$, long. $98^{\circ} 15^{\prime} 59^{\prime \prime}, S^{\prime} 1 / 4$ sec.15, T.8 N., R.10 W., 2 miles south of Gracemont, Oklahoma.

\begin{tabular}{|c|c|c|c|c|c|c|c|}
\hline Date & $\begin{array}{c}\text { Discharge } \\
\left(\mathrm{ft}^{3} / \mathrm{s}\right)\end{array}$ & Date & $\begin{array}{c}\text { Discharge } \\
\left(\mathrm{ft}^{3} / \mathrm{s}\right)\end{array}$ & Date & $\begin{array}{c}\text { Discharge } \\
\left(\mathrm{ft}^{3} / \mathrm{s}\right)\end{array}$ & Date & $\begin{array}{c}\text { Discharge } \\
\left(\mathrm{ft}^{3} / \mathrm{s}\right)\end{array}$ \\
\hline $\begin{array}{r}12-20-49 \\
9-5-51 \\
11-29-51 \\
1-16-52 \\
3-5-52 \\
4-30-52 \\
6-4-52 \\
7-23-52 \\
10-7-52 \\
11-3-52\end{array}$ & $\begin{array}{l}7.88 \\
0 \\
2.89 \\
3.90 \\
2.53 \\
15.9 \\
8.45 \\
0 \\
0 \\
0\end{array}$ & $\begin{array}{r}12-12-52 \\
1-20-53 \\
3-3-53 \\
3-30-53 \\
5-19-53 \\
6-22-53 \\
7-28-53 \\
8-25-53 \\
10-2-53 \\
11-3-53\end{array}$ & $\begin{array}{l}0 \\
6.19 \\
19.0 \\
16.8 \\
5.75 \\
0 \\
3.50 \\
4.84 \\
0 \\
5.68\end{array}$ & $\begin{array}{r}12-2-53 \\
1-19-54 \\
2-16-54 \\
3-16-54 \\
5-6-54 \\
6-4-54 \\
6-30-54 \\
8-3-54 \\
9-8-54 \\
10-19-54\end{array}$ & $\begin{array}{l}7.34 \\
9.68 \\
6.43 \\
7.40 \\
7.60 \\
5.89 \\
0 \\
0 \\
0 \\
0\end{array}$ & $\begin{array}{r}11-16-54 \\
12-16-54 \\
1-18-55 \\
2-15-55 \\
3-17-55 \\
4-20-55 \\
6-16-55 \\
8-20-55 \\
8-22-55\end{array}$ & $\begin{array}{l}0 \\
0 \\
.70 \\
.76 \\
0 \\
1.29 \\
6.62 \\
0 \\
0\end{array}$ \\
\hline
\end{tabular}

Suttle Creek near Davidson, Oklahoma, 07308310 , partial record site.

Lat. $34^{\circ} 15^{\prime} 00^{\prime \prime}$, long. $90^{\circ} 05^{\prime} 00^{\prime \prime}$, south line of SW $1 / 4$ SE $1 / 4 \mathrm{sec} .31$, T.3 S., R.12 W., at bridge on U.S. Highway $70,3-1 / 4$ miles east of Davidson and about 4 miles upstream from mouth.

\begin{tabular}{|c|c|c|c|c|c|c|c|}
\hline Date & $\begin{array}{c}\text { Discharge } \\
\left(\mathrm{ft}^{3} / \mathrm{s}\right)\end{array}$ & Date & $\begin{array}{c}\text { Discharge } \\
\left(\mathrm{ft}^{3} / \mathrm{s}\right)\end{array}$ & Date & $\begin{array}{c}\text { Discharge } \\
\left(\mathrm{ft}^{3} / \mathrm{s}\right)\end{array}$ & Date & $\begin{array}{c}\text { Discharge } \\
\left(\mathrm{ft}^{3} / \mathrm{s}\right)\end{array}$ \\
\hline $\begin{array}{l}10-27-64 \\
12-15-64\end{array}$ & 0.37 & $\begin{array}{l}3-1-65 \\
7-8-65\end{array}$ & $0^{.11}$ & $\begin{array}{r}11-30-65 \\
1-11-66\end{array}$ & $\begin{array}{l}.11 \\
.09\end{array}$ & $\begin{array}{l}3-23-66 \\
6-28-66\end{array}$ & $0^{.01}$ \\
\hline
\end{tabular}


Sweetwater Creek near Texola, Oklahoma, Miscellaneous site. Lat. $35^{\circ} 18^{\prime} 30^{\prime \prime}$, long. $99^{\circ} 58^{\prime} 00^{\prime \prime}, \mathrm{NW} 1 / 4 \mathrm{sec} .28$, T. $10 \mathrm{~N}$, , R. 26 W., at county highway bridge, $7 \mathrm{miles}$ north of Texola, Oklahoma.

\begin{tabular}{|c|c|c|c|c|c|c|c|}
\hline Date & $\begin{array}{c}\text { Discharge } \\
\left(\mathrm{ft}^{3} / \mathrm{s}\right)\end{array}$ & Date & $\begin{array}{c}\text { Discharge } \\
\left(\mathrm{ft}^{3} / \mathrm{s}\right)\end{array}$ & Date & $\begin{array}{c}\text { Discharge } \\
\left(\mathrm{ft}^{3} / \mathrm{s}\right)\end{array}$ & Date & $\begin{array}{c}\text { Discharge } \\
\left(\mathrm{ft}^{3} / \mathrm{s}\right)\end{array}$ \\
\hline $\begin{array}{r}9-24-51 \\
10-28-51 \\
12-11-51 \\
2-6-52 \\
3-24-52\end{array}$ & $\begin{array}{r}1.55 \\
.05 \\
11.9 \\
15.5 \\
10.2\end{array}$ & $\begin{array}{l}6-12-52 \\
7-28-52 \\
8-20-52 \\
1-6-53\end{array}$ & $\begin{array}{l}1.63 \\
0 \\
0 \\
2.06\end{array}$ & $\begin{array}{r}2-6-53 \\
3-11-53 \\
9-29-53 \\
11-16-53\end{array}$ & $\begin{array}{l}7.11 \\
5.17 \\
0 \\
3.44\end{array}$ & $\begin{array}{r}12-21-53 \\
1-14-54 \\
3-16-54 \\
10-19-54 \\
12-23-54\end{array}$ & $\begin{array}{l}3.36 \\
7.98 \\
3.66 \\
0 \\
0\end{array}$ \\
\hline
\end{tabular}

Sycamore Creek near Wyandotte, Oklahoma, Miscellaneous site.

Lat. $36^{\circ} 52^{\prime} 50^{\prime \prime}$, long. $94^{\circ} 36^{\prime} 43^{\prime \prime}$, SE $1 / 4 \mathrm{SW} 1 / 4$ sec.35, T.27 N., R.25 E., 200 ft upstream from bridge on State Highway 10 , and $2-1 / 2$ miles southeast of Wyandotte, Okla.
Date
Discharge
$3-9-54$
5.18

Tenmile Creek near Miller, Oklahoma, 07336000 , partial record site.

Lat. $34^{\circ} 17^{\prime} 55^{\prime \prime}$, long. $95^{\circ} 44^{\prime} 40^{\prime \prime}$, in NW $1 / 4^{\prime}$ sec.16, T.3 S., R.15 E., Pushmataha County, at county road bridge, 1.2 miles south of Miller, and at mile 11.6.

\begin{tabular}{|c|c|c|c|c|c|c|c|}
\hline Date & $\begin{array}{c}\text { Discharge } \\
\left(\mathrm{ft}^{3} / \mathrm{s}\right)\end{array}$ & Date & $\begin{array}{c}\text { Discharge } \\
\left(\mathrm{ft}^{3} / \mathrm{s}\right)\end{array}$ & Date & $\begin{array}{c}\text { Discharge } \\
\left(\mathrm{ft}^{3} / \mathrm{s}\right)\end{array}$ & Date & $\begin{array}{c}\text { Discharge } \\
\left(\mathrm{ft}^{3} / \mathrm{s}\right)\end{array}$ \\
\hline $\begin{array}{l}3-11-71 \\
6-22-71 \\
7-28-71\end{array}$ & $\begin{array}{r}28.0 \\
4.3 \\
2.9\end{array}$ & $\begin{array}{l}8-31-71 \\
1-11-72\end{array}$ & $\begin{array}{c}0 \\
24.0\end{array}$ & $\begin{array}{l}6-28-72 \\
8-23-72\end{array}$ & $\begin{array}{l}1.8 \\
0\end{array}$ & $\begin{array}{r}12-6-72 \\
5-22-73\end{array}$ & $\begin{array}{l}10 . \\
4.5\end{array}$ \\
\hline
\end{tabular}

Tepee Creek near Eva, Oklahoma, 07232450, partial record site.

Lat. $36^{\circ} 42^{\prime} 30^{\prime \prime}$, long. $101^{\circ} 43^{\prime} 30^{\prime \prime}$, on west line sec. 29 , T.3 N., R.13 E., Texas County, at low water crossing on county road, 8 miles northwest of Goodweli, Okla.

\begin{tabular}{|c|c|c|c|c|c|c|c|}
\hline Date & $\begin{array}{c}\text { Discharge } \\
\left(\mathrm{ft}^{3} / \mathrm{s}\right)\end{array}$ & Date & $\begin{array}{c}\text { Discharge } \\
\left(\mathrm{ft}^{3} / \mathrm{s}\right)\end{array}$ & Date & $\begin{array}{c}\text { Discharge } \\
\left(\mathrm{ft}^{3} / \mathrm{s}\right)\end{array}$ & Date & $\begin{array}{c}\text { Discharge } \\
\left(\mathrm{ft}^{3} / \mathrm{s}\right)\end{array}$ \\
\hline $\begin{array}{r}11-23-66 \\
1-16-67 \\
4-24-67 \\
7-24-67 \\
10-16-67 \\
2-26-68 \\
5-20-68\end{array}$ & $\begin{array}{l}.22 \\
.32 \\
.14 \\
.20 \\
.14 \\
.20 \\
.20\end{array}$ & $\begin{array}{r}7-23-68 \\
12-24-68 \\
3-27-69 \\
5-27-69 \\
8-12-69 \\
12-3-69 \\
3-9-70\end{array}$ & $\begin{array}{r}.21 \\
.38 \\
.20 \\
.20 \\
0 . \\
.18 \\
.25\end{array}$ & $\begin{array}{r}5-19-70 \\
7-30-70 \\
11-10-70 \\
3-1-71 \\
6-15-71 \\
9-27-71 \\
11-8-71\end{array}$ & $\begin{array}{l}.21 \\
.02 \\
.00 \\
.20 \\
.20 \\
.01 \\
.51\end{array}$ & $\begin{array}{r}3-1-72 \\
5-0-72 \\
8-7-72 \\
12-18-72 \\
3-7-73 \\
5-30-73 \\
7-8-49\end{array}$ & $\begin{array}{l}.21 \\
.07 \\
.15 \\
.11 \\
.14 \\
.38\end{array}$ \\
\hline
\end{tabular}

Threemile Creek near Mill Creek, Oklahoma, Miscellaneous site.

Lat. $34^{\circ} 29^{\prime} 15^{\prime \prime}$, long. $96^{\circ} 50^{\prime} 00^{\prime \prime}$, NE $1 / 4 \mathrm{sec} .12$, T.2 S., R.4 E., at county highway bridge, $100 \mathrm{ft}$ below St. Louis \& San Francisco Railway dam and $1 / 4$ mile west of Mill Creek, Okla.

\begin{tabular}{|c|c|c|c|c|c|c|c|}
\hline Date & $\begin{array}{c}\text { Discharge } \\
\left(\mathrm{ft}^{3} / \mathrm{s}\right)\end{array}$ & Date & $\begin{array}{c}\text { Discharge } \\
\left(\mathrm{ft}^{3} / \mathrm{s}\right)\end{array}$ & Date & $\begin{array}{c}\text { Discharge } \\
\left(\mathrm{ft}^{3} / \mathrm{s}\right)\end{array}$ & Date & $\begin{array}{c}\text { Discharge } \\
\left(\mathrm{ft}^{3} / \mathrm{s}\right)\end{array}$ \\
\hline $\begin{array}{r}9-10-51 \\
11-27-51\end{array}$ & $\begin{array}{l}.12 \\
.52\end{array}$ & $\begin{array}{l}1-15-52 \\
3-5-52\end{array}$ & $\begin{array}{l}.26 \\
.48\end{array}$ & $\begin{array}{l}4-23-52 \\
6-11-52\end{array}$ & $\begin{array}{r}1.61 \\
.30\end{array}$ & $\begin{array}{l}7-22-52 \\
9-\quad 3-52\end{array}$ & $\begin{array}{l}.16 \\
.01\end{array}$ \\
\hline
\end{tabular}

Timber Creek near Sayre, Oklahoma, Miscellaneous site.

Lat. $35^{\circ} 177^{\prime \prime}$, long. $99^{\circ} 34^{\prime} 29^{\prime \prime}$, on north line, sec.1, T.9 N., R.23 W., at bridge on State Highway 152,3 miles east of Sayre, Okla.

$\begin{array}{rr}\text { Date } & \begin{array}{c}\text { Discharge } \\ \left(\mathrm{ft}^{3} / \mathrm{s}\right)\end{array} \\ 2-14-57 & .18\end{array}$


Travertine Creek at Sulphur, Oklahoma, Miscellaneous site.

Lat. $34^{\circ} 30^{\prime} 14^{\prime \prime}$, long. $96^{\circ} 58^{\prime} 52^{\prime \prime}$, in NE $1 / 4 \mathrm{NE} 1 / 4 \mathrm{sec} .3$, T.1 S., R.3 E., at mouth of creek in Platt National Park, $1 / 2$ mile south of Sulphur, Okla.

\begin{tabular}{|c|c|c|c|c|c|c|c|}
\hline Date & $\begin{array}{c}\text { Discharge } \\
\left(\mathrm{ft}^{3} / \mathrm{s}\right)\end{array}$ & Date & $\begin{array}{c}\text { Discharge } \\
\left(\mathrm{ft}^{3} / \mathrm{s}\right)\end{array}$ & Date & $\begin{array}{c}\text { Discharge } \\
\left(\mathrm{ft}^{3} / \mathrm{s}\right)\end{array}$ & Date & $\begin{array}{c}\text { Discharge } \\
\left(\mathrm{ft}^{3} / \mathrm{s}\right)\end{array}$ \\
\hline$-18-58$ & 12.8 & $10-30-58$ & 5.75 & $2-19-59$ & 2.78 & $7-15-59$ & 2.16 \\
\hline
\end{tabular}

Turkey Creek near Camargo, Oklahoma, 07228220 , partial record site.

Lat. $36^{\circ} 02^{\prime} 30^{\prime \prime}$, long. $99^{\circ} 24^{\prime} 30^{\prime \prime}$, in NE $1 / 4$ NW $1 / 4 \mathrm{sec} .14, \mathrm{~T} .18 \mathrm{~N} ., \mathrm{R} .21 \mathrm{~W}$. , Ellis County, at pasture 0.1 mile south of county road, 7.5 miles northwest of Camargo, and about 3.5 miles above mouth.

$\begin{array}{rccccccc}\text { Date } & \begin{array}{c}\text { Discharge } \\ \left(\mathrm{ft}^{3} / \mathrm{s}\right)\end{array} & \text { Date } & \begin{array}{c}\text { Discharge } \\ \left(\mathrm{ft}^{3} / \mathrm{s}\right)\end{array} & \text { Date } & \begin{array}{c}\text { Discharge } \\ \left(\mathrm{ft}^{3} / \mathrm{s}\right)\end{array} & \begin{array}{c}\text { Date } \\ \text { Discharge } \\ \left(\mathrm{ft}^{3} / \mathrm{s}\right)\end{array} \\ 10-26-64 & 0 & 5-18-67 & 1.3 & 6-20-69 & 3.0 & 12-17-71 & 1.2 \\ 3-26-65 & 2.12 & 9-1-67 & 0 & 12-16-69 & 2.0 & 3-17-72 & 1.5 \\ 7-9-65 & 2.30 & 1-15-68 & 1.3 & 2-19-70 & 1.7 & 6-2-72 & 0 \\ 11-9-65 & 2.5 & 4-1-68 & 1.4 & 6-11-70 & 0 & 8-18-72 & 0 \\ 2-17-66 & 5.4 & 7-9-68 & 0.06 & 11-6-70 & 0 & 12-20-72 & 3.2 \\ 5-12-66 & 1.5 & 9-16-68 & 0 & 3-4-71 & 2.1 & 3-2-73 & 2.2 \\ 11-23-66 & .88 & 12-17-68 & 1.5 & 5-27-71 & 0 & 6-15-73 & .21 \\ 2-17-67 & 1.8 & 4-4-69 & 2.8 & 9-8-71 & 0 & & \end{array}$

Turkey Creek near Clinton, Oklahoma, Miscellaneous site.

Lat. $35^{\circ} 25^{\prime} 00^{\prime \prime}$, long. $99^{\circ} 15^{\prime} 00^{\prime \prime}$, SW $1 / 4 \mathrm{sec} .18, \mathrm{~T} .11 \mathrm{~N} ., \mathrm{R} .19 \mathrm{~W} ., 1 \cdot 1 / 2 \mathrm{miles}$ east of Canute and 17 miles southwest of Ciinton, okla.

\begin{tabular}{|c|c|c|c|c|c|c|c|}
\hline Date & $\begin{array}{c}\text { Dis charge } \\
\left(\mathrm{ft}^{3} / \mathrm{s}\right)\end{array}$ & Date & $\begin{array}{c}\text { Discharge } \\
\left(\mathrm{ft}^{3} / \mathrm{s}\right)\end{array}$ & Date & $\begin{array}{c}\text { Discharge } \\
\left(\mathrm{ft}^{3} / \mathrm{s}\right)\end{array}$ & Date & $\begin{array}{c}\text { Discharge } \\
\left(\mathrm{ft}^{3} / \mathrm{s}\right)\end{array}$ \\
\hline $\begin{array}{r}12-1-54 \\
1-17-55\end{array}$ & $\begin{array}{l}.43 \\
.72\end{array}$ & $\begin{array}{l}1-24-55 \\
3-\quad 1-55\end{array}$ & $\begin{array}{l}.76 \\
.59\end{array}$ & $\begin{array}{l}4-19-55 \\
3-20-56\end{array}$ & $\begin{array}{l}.43 \\
.49\end{array}$ & $9-10-56$ & 0 \\
\hline
\end{tabular}

Turkey Creek near Dover, Oklahoma, Miscellaneous site.

Lat. $36^{\circ} 00^{\prime} 00^{\prime \prime}$, long. $97^{\circ} 45^{\prime} 00^{\prime \prime}$, NW $1 / 4 \mathrm{sec} .2$, T.17 N., R.7 W., at county highway bridge at Dover, oklahoma.

$\begin{array}{rrrrrrrr}\text { Date } & \begin{array}{c}\text { Discharge } \\ \left(\mathrm{ft}^{3} / \mathrm{s}\right)\end{array} & \text { Date } & \begin{array}{c}\text { Discharge } \\ \left(\mathrm{ft}^{3} / \mathrm{s}\right)\end{array} & \text { Date } & \begin{array}{c}\text { Discharge } \\ \left(\mathrm{ft}^{3} / \mathrm{s}\right)\end{array} & \begin{array}{c}\text { Date } \\ 7-27-49\end{array} \\ 7.64 & 1-8-52 & 7.99 & 7-21-52 & .04 & 2-2-53 & \begin{array}{c}\text { Discharge } \\ \left(\mathrm{ft}^{3} / \mathrm{s}^{2}\right)\end{array} \\ 9-29-49 & 3.24 & 2-5-52 & 6.18 & 9-3-52 & .06 & 3-53 & 4.80 \\ 10-11-50 & 14.8 & 3-3-52 & 9.40 & 10-14-52 & .15 & 4-20-53 & 1.96 \\ 9-19-51 & 8.19 & 4-14-52 & 7.48 & 11-4-52 & .12 & 5-14-53 & 1.59 \\ 10-2-51 & 4.94 & 4-24-52 & 157.0 & 12-1-52 & .25 & 8-5-53 & 1.99 \\ 10-29-51 & 11.2 & 6-4-52 & 4.94 & 1-5-53 & .42 & 8-31-53 & 0.32 \\ 12-10-51 & 10.5 & & & & & 10-14-53 & 0\end{array}$

Turkey Creek near Drummond, Oklahoma, 07159000 , partial record site.

Lat. $36^{\circ} 19^{\circ} 05^{\prime \prime}$, long. $98^{\circ} 00^{\prime} 03^{\prime \prime}$, in NE $1 / 4 \mathrm{NE} 1 / 4 \mathrm{sec} .12, \mathrm{~T} .21 \mathrm{~N} ., \mathrm{R} .8 \mathrm{~K}$., Garfield County, at county road bridge, 2.2 miles northeast of Drummond, Okla.

\begin{tabular}{|c|c|c|c|c|c|c|c|}
\hline Date & $\begin{array}{c}\text { Discharge } \\
\left(\mathrm{ft}^{3} / \mathrm{s}\right)\end{array}$ & Date & $\begin{array}{c}\text { Discharge } \\
\left(\mathrm{ft}^{3} / \mathrm{s}\right)\end{array}$ & Date & $\begin{array}{c}\text { Discharge } \\
\left(\mathrm{ft}^{3} / \mathrm{s}\right)\end{array}$ & Date & $\begin{array}{c}\text { Discharge } \\
\left(\mathrm{ft}^{3} / \mathrm{s}\right)\end{array}$ \\
\hline $\begin{array}{r}12-2-70 \\
3-23-71 \\
5-7-71\end{array}$ & $\begin{array}{l}.47 \\
1.5 \\
1.9\end{array}$ & $\begin{array}{r}9-8-71 \\
11-11-71 \\
3-9-72\end{array}$ & $\begin{array}{l}.06 \\
1.8 \\
1.4\end{array}$ & $\begin{array}{r}4-19-72 \\
8-1-72 \\
10-26-72\end{array}$ & $\begin{array}{l}3.6 \\
.45 \\
.02\end{array}$ & $\begin{array}{l}2-28-73 \\
5-23-73\end{array}$ & $\begin{array}{r}4.5 \\
43 .\end{array}$ \\
\hline
\end{tabular}


Turkey Creek near Hennessey, Oklahoma, Miscellaneous site.

Lat. $36^{\circ} 07^{\prime} 45^{\prime \prime}$, long. $97^{\circ} 54^{\prime} 40^{\prime \prime}$, north ine sec.23, T.ig N., R. 7 W., at bridge on State Highway 51 , $1-1 / 2$ miles west of Hennessey, okla.

$\begin{array}{cccr}\text { Date } & \begin{array}{c}\text { Discharge } \\ \left(\mathrm{ft}^{3} / \mathrm{s}\right)\end{array} & \text { Date } & \begin{array}{r}\text { Discharge } \\ \left(\mathrm{ft}^{3} / \mathrm{s}\right)\end{array} \\ 12-6-48 & 10.9 & 11-13-50 & 8.55\end{array}$

Turkey Creek at 0lustee, Oklahoma, 07301100 , partial record site.

Lat. $34^{\circ} 35^{\prime} 58^{\prime \prime}$, long. $99^{\circ} 26^{\prime} 12^{\prime \prime}$, in NW 1/4 sec.5, T.1 N., R.21 W., Jackson County, at county road bridge, 3.2 miles north of olustee, and at mile 6.4 .

\begin{tabular}{|c|c|c|c|c|c|c|c|}
\hline Date & $\begin{array}{c}\text { Discharge } \\
\left(\mathrm{ft}^{3} / \mathrm{s}\right)\end{array}$ & Date & $\begin{array}{c}\text { Discharge } \\
\left(\mathrm{ft}^{3} / \mathrm{s}\right)\end{array}$ & Date & $\begin{array}{c}\text { Discharge } \\
\left(\mathrm{ft}^{3} / \mathrm{s}\right)\end{array}$ & Date & $\begin{array}{c}\text { Discharge } \\
\left(\mathrm{ft}^{3} / \mathrm{s}\right)\end{array}$ \\
\hline $\begin{array}{r}9-24-51 \\
1-15-52 \\
3-17-52 \\
6-10-52 \\
7-24-52 \\
8-26-52 \\
9-29-52 \\
11-13-52 \\
12-18-52 \\
1-19-53 \\
5-15-53 \\
9-29-53\end{array}$ & $\begin{array}{l}6.81 \\
5.19 \\
6.34 \\
5.64 \\
3.72 \\
1.60 \\
1.06 \\
2.41 \\
2.60 \\
4.36 \\
2.29 \\
0\end{array}$ & $\begin{array}{r}11-16-53 \\
12-18-53 \\
3-25-54 \\
8-20-54 \\
10-21-54 \\
12-14-54 \\
1-14-55 \\
2-16-55 \\
4-19-55 \\
7-14-55 \\
9-6-55 \\
1-5-56\end{array}$ & $\begin{array}{l}1.99 \\
2.10 \\
.94 \\
0 \\
0 \\
0 \\
0 \\
.94 \\
0.90 \\
0.10 \\
4.38\end{array}$ & $\begin{array}{r}2-15-56 \\
3-15-56 \\
4-18-56 \\
6-18-56 \\
7-19-56 \\
9-6-56 \\
10-1-56 \\
12-4-56 \\
1-4-57 \\
2-20-57 \\
3-19-57 \\
4-16-57\end{array}$ & $\begin{array}{r}3.69 \\
3.83 \\
1.90 \\
2.21 \\
12.8 \\
.88 \\
.86 \\
2.97 \\
4.94 \\
3.81 \\
2.93 \\
2.69\end{array}$ & $\begin{array}{r}6-12-57 \\
7-18-57 \\
8-6-70 \\
10-7-70 \\
1-20-71 \\
8-4-71 \\
12-22-71 \\
2-17-72 \\
4-12-72 \\
7-19-72 \\
11-6-72 \\
2-8-73\end{array}$ & 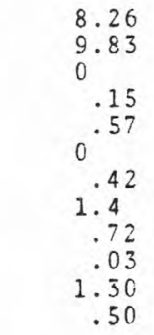 \\
\hline
\end{tabular}

Turkey Creek near Roll, Oklahoma, Miscellaneous site.

Lat. $35^{\circ} 48^{\prime} 41^{\prime \prime}$, long. $99^{\circ} 53^{\prime} 50^{\prime \prime}$, NW 1/4 sec.6, T. 15 N., R. 25 H., at bridge on State Highway 33 , 11 miles west of Roll, Okla.

$\begin{array}{cc}\text { Date } & \begin{array}{c}\text { Discharge } \\ \left(\mathrm{ft}^{3} / \mathrm{s}\right)\end{array} \\ 9-13-55 & 0\end{array}$

Turkey Creek near Sayre, Oklahoma (Big Turkey Creek), 07301460 , partial record site.

Lat. $35^{\circ} 15^{\prime} 35^{\prime \prime}$, long. $99^{\circ} 43^{\prime} 20^{\prime \prime}$, in NE 1/4 NE 1/4 sec.15, T.9. N., R. 24 H. Beckham County, at east.

bound bridge on U.S. Highway $66,5.5$ miles southwest of Sayre, and about 1 mile upstream from mouth.

\begin{tabular}{|c|c|c|c|c|c|c|c|}
\hline Date & $\begin{array}{c}\text { Discharge } \\
\left(\mathrm{ft}^{3} / \mathrm{s}\right)\end{array}$ & Date & $\begin{array}{c}\text { Discharge } \\
\left(\mathrm{ft}^{3} / \mathrm{s}\right)\end{array}$ & Date & $\begin{array}{c}\text { Discharge } \\
\left(\mathrm{ft}^{3} / \mathrm{s}\right)\end{array}$ & Date & $\begin{array}{c}\text { Discharge } \\
\left(f t^{3} / \mathrm{s}\right)\end{array}$ \\
\hline $\begin{array}{r}12-9-53 \\
2-3-54 \\
3-9-54 \\
4-20-54 \\
7-2-54 \\
8-30-54 \\
11-16-54 \\
12-16-54 \\
2-28-55 \\
3-30-55 \\
4-28-55\end{array}$ & $\begin{array}{r}1.13 \\
.62 \\
1.23 \\
1.15 \\
.80 \\
.23 \\
.71 \\
.71 \\
.90 \\
.76 \\
.63\end{array}$ & $\begin{array}{r}7-12-55 \\
8-30-55 \\
11-14-56 \\
11-9-65 \\
2-7-66 \\
5-9-66 \\
11-22-66 \\
2-16-67 \\
5-23-67 \\
9-1-67\end{array}$ & $\begin{array}{l}.26 \\
.99 \\
.23 \\
2.3 \\
2.0 \\
2.1 \\
1.5 \\
1.2 \\
.88 \\
.50\end{array}$ & $\begin{array}{r}12-13-67 \\
3-28-68 \\
6-26-68 \\
9-16-68 \\
12-11-68 \\
4-4-69 \\
6-11-69 \\
12-16-69 \\
2-17-70 \\
6-11-70\end{array}$ & $\begin{array}{l}.88 \\
1.0 \\
1.4 \\
.82 \\
2.1 \\
1.7 \\
2.5 \\
1.4 \\
2.1 \\
.94\end{array}$ & $\begin{array}{r}8-12-70 \\
11-25-70 \\
1-27-71 \\
5-20-71 \\
8-12-71 \\
10-14-71 \\
3-7-72 \\
4-18-72 \\
11-14-72 \\
2-7-73 \\
5-30-73\end{array}$ & $\begin{array}{r}.46 \\
.47 \\
2.9 \\
1.1 \\
.16 \\
.28 \\
.87 \\
.68 \\
.71 \\
1.10 \\
1.60\end{array}$ \\
\hline
\end{tabular}

Two Hatchett Creek near Fort Cobb, Oklahoma, Miscellaneous site.

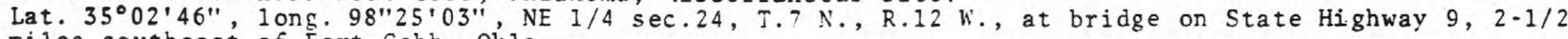
miles southeast of Fort Cobb, okla.

$\begin{array}{cr}\text { Date } & \begin{array}{r}\text { Discharge } \\ \left(\mathrm{ft}^{3} / \mathrm{s}\right)\end{array} \\ 1-9-52 & .08\end{array}$


Vendome Spring near Sulphur, Oklahoma, Miscellaneous site.

Lat. $34^{\circ} 30^{\prime} 14^{\prime \prime}$, long. $96^{\circ} 58^{\prime} 52^{\prime \prime}$, SE $1 / 4 \mathrm{NE} 1 / 4 \mathrm{NE} 1 / 4 \mathrm{sec} .3$, T.1 S., R. 3 E., in Platt National Park, $1 / 4 \mathrm{mile}$ south of Sulphur, Okla.

$\begin{array}{cr}\text { Date } & \begin{array}{r}\text { Discharge } \\ \left(\mathrm{ft}^{3} / \mathrm{s}\right)\end{array} \\ 11-15-55 & 1.70\end{array}$

Walnut Creek near Purcell, Oklahoma 07229300 , partial record site.

Lat. $34^{\circ} 59^{\prime} 56^{\prime \prime}$, long. $97^{\circ} 22^{\prime} 00^{\prime \prime}$, NW $1 / 4 \mathrm{sec} .13$, T.6 N., R.2 W., at bridge on U.S. Highway 77 at Purcell, Okla. Continuous record $65-72$.

\begin{tabular}{|c|c|c|c|c|c|c|c|}
\hline Date & $\begin{array}{c}\text { Discharge } \\
\left(\mathrm{ft}^{3} / \mathrm{s}\right)\end{array}$ & Date & $\begin{array}{c}\text { Discharge } \\
\left(\mathrm{ft}^{3} / \mathrm{s}\right)\end{array}$ & Date & $\begin{array}{c}\text { Discharge } \\
\left(\mathrm{ft}^{3} / \mathrm{s}\right)\end{array}$ & Date & $\begin{array}{c}\text { Discharge } \\
\left(\mathrm{ft}^{3} / \mathrm{s}\right)\end{array}$ \\
\hline $\begin{array}{r}9-5-51 \\
10-9-51 \\
11-26-51 \\
1-14-52 \\
3-3-52 \\
4-21-52 \\
6-13-52 \\
7-24-52 \\
9-13-52 \\
9-4-52 \\
11-18-53 \\
1-29-54 \\
2-25-54 \\
4-19-54\end{array}$ & $\begin{array}{c}5.21 \\
6.28 \\
20.4 \\
6.38 \\
90.8 \\
84.7 \\
29.2 \\
2.10 \\
0 \\
.60 \\
7.95 \\
12.1 \\
6.79 \\
5.13\end{array}$ & $\begin{array}{r}5-28-54 \\
7-14-54 \\
8-24-54 \\
9-15-54 \\
11-2-54 \\
12-8-54 \\
2-1-55 \\
2-28-55 \\
6-4-55 \\
7-28-55 \\
9-20-55 \\
1-16-58 \\
3-20-58 \\
6-12-58\end{array}$ & $\begin{array}{c}13.5 \\
0 \\
0 \\
0 \\
0 \\
0 \\
4.74 \\
3.74 \\
3.25 \\
0 \\
0 \\
48.9 \\
18.2 \\
7.39\end{array}$ & $\begin{array}{r}9-2-58 \\
10-31-58 \\
1-9-59 \\
6-9-59 \\
9-14-59 \\
10-26-59 \\
12-7-59 \\
9-13-60 \\
12-9-60 \\
2-2-61 \\
3-9-61 \\
6-2-61 \\
6-30-61 \\
7-20-61\end{array}$ & $\begin{array}{c}3.01 \\
16.5 \\
14.2 \\
18.2 \\
4.68 \\
9.21 \\
19.5 \\
7.85 \\
24.8 \\
24.9 \\
16.5 \\
12.4 \\
13.1 \\
14.0\end{array}$ & $\begin{array}{r}12-20-61 \\
2-21-62 \\
5-14-62 \\
8-21-62 \\
1-3-63 \\
5-20-63 \\
7-22-63 \\
10-11-63 \\
6-12-64 \\
7-14-64 \\
9-15-64 \\
10-20-64 \\
3-8-65 \\
7-13-65\end{array}$ & $\begin{array}{r}70.4 \\
16.4 \\
4.26 \\
2.29 \\
21.8 \\
17.3 \\
.20 \\
.07 \\
.66 \\
0.14 \\
.14 \\
.18 \\
8.76 \\
.23\end{array}$ \\
\hline
\end{tabular}

West Bitter Creek near Chickasha, Oklahoma, Miscellaneous site.

Lat. $35^{\circ} 02 \cdot 30^{\prime \prime}$, long. $97^{\circ} 51^{\prime} 00^{\prime \prime}, \mathrm{NW} 1 / 4 \mathrm{NW} 1 / 4 \mathrm{sec} .32, \mathrm{~T} .7 \mathrm{~N} ., \mathrm{R} .6 \mathrm{~W}$. , at bridge on U.S. Highway 62 , 4 miles east of Chickasha, Okla.

\begin{tabular}{|c|c|c|c|c|c|c|c|}
\hline Date & $\begin{array}{c}\text { Discharge } \\
\left(\mathrm{ft}^{3} / \mathrm{s}\right)\end{array}$ & Date & $\begin{array}{c}\text { Discharge } \\
\left(\mathrm{ft}^{3} / \mathrm{s}\right)\end{array}$ & Date & $\begin{array}{c}\text { Discharge } \\
\left(\mathrm{ft}^{3} / \mathrm{s}\right)\end{array}$ & Date & $\begin{array}{c}\text { Discharge } \\
\left(\mathrm{ft}^{3} / \mathrm{s}\right)\end{array}$ \\
\hline $\begin{array}{l}6-4-54 \\
6-30-54 \\
8-3-54 \\
9-8-54\end{array}$ & $0_{0}^{.71}$ & $\begin{array}{l}10-19-54 \\
11-16-54 \\
12-16-54\end{array}$ & $\begin{array}{r}.14 \\
.32 \\
.64\end{array}$ & $\begin{array}{l}1-18-55 \\
2-15-55 \\
3-17-55\end{array}$ & $\begin{array}{r}17.5 \\
.72 \\
.57\end{array}$ & $\begin{array}{l}4-20-55 \\
6-16-55 \\
8-1-55 \\
8-22-55\end{array}$ & $\begin{array}{r}.45 \\
1.70 \\
.04\end{array}$ \\
\hline
\end{tabular}

West Bitter Creek near Tabler, Oklahoma, Miscellaneous site. Lat. $35^{\circ} 03^{\prime} 30^{\prime \prime}$, long. $97^{\circ} 5 i^{\prime} 00^{\prime \prime}$, SE $1 / 4 \mathrm{sec} .20$, T. $7 \mathrm{~N} ., \mathrm{R} .6 \mathrm{w}$. , at county highway bridge, 2 miles northwest of Tabler, okla.

\begin{tabular}{|c|c|c|c|c|c|c|c|}
\hline Date & $\begin{array}{c}\text { Discharge } \\
\left(\mathrm{ft}^{3} / \mathrm{s}\right)\end{array}$ & Date & $\begin{array}{c}\text { Discharge } \\
\left(\mathrm{ft}^{3} / \mathrm{s}\right)\end{array}$ & Date & $\begin{array}{c}\text { Discharge } \\
\left(\mathrm{ft}^{3} / \mathrm{s}\right)\end{array}$ & Date & $\begin{array}{c}\text { Discharge } \\
\left(\mathrm{ft}^{3} / \mathrm{s}\right)\end{array}$ \\
\hline $\begin{array}{r}7-10-52 \\
8-20-52 \\
10-7-52\end{array}$ & $\begin{array}{l}0^{.13} \\
0\end{array}$ & $\begin{array}{r}11-3-52 \\
12-12-52 \\
1-20-53\end{array}$ & $\begin{array}{r}.29 \\
1.46 \\
1.91\end{array}$ & $\begin{array}{l}3-3-53 \\
3-30-53 \\
5-19-53\end{array}$ & $\begin{array}{r}231.0 \\
3.90 \\
2.32\end{array}$ & $\begin{array}{l}6-7-53 \\
7-28-53 \\
8-25-53\end{array}$ & $0^{0} .22$ \\
\hline
\end{tabular}

West Bitter Creek near Tabler, Oklahoma, Miscellaneous site.

Lat. $35^{\circ} 05^{\prime} 46^{\prime \prime}$, long. $97^{\circ} 49^{\prime} 54^{\prime \prime}$, SW $1 / 4$ sec.9, T.7 N., R. 6 W., 3 miles north of Tabler, Okla.

\section{Date Discharge

$$
\left(\mathrm{ft}^{3} / \mathrm{s}\right)
$$

5- $3-50$
.80 
West Cache Creek near Cookietown, Oklahoma, 073112400 , partial record site.

Lat. $34^{\circ} 16^{\prime} 30^{\prime \prime}$, long. $98^{\circ} 23^{\prime} 15^{\prime \prime}$, in NE $1 / 4 \mathrm{sec} .30, \mathrm{~T} .3 \mathrm{~S} .$, R.11 W., Cotton County, at bridge on State Highway $5 \mathrm{~A}, 3.5$ miles east of Cookietown, and about 11 miles upstream from mouth.

\begin{tabular}{|c|c|c|c|c|c|c|c|}
\hline Date & $\begin{array}{c}\text { Discharge } \\
\left(\mathrm{ft}^{3} / \mathrm{s}\right)\end{array}$ & Date & $\begin{array}{c}\text { Discharge } \\
\left(\mathrm{ft}^{3} / \mathrm{s}\right)\end{array}$ & Date & $\begin{array}{c}\text { Discharge } \\
\left(\mathrm{ft}^{3} / \mathrm{s}\right)\end{array}$ & Date & $\begin{array}{c}\text { Discharge } \\
\left(\mathrm{ft}^{3} / \mathrm{s}\right)\end{array}$ \\
\hline $\begin{array}{r}1-14-52 \\
6-11-52 \\
8-21-52 \\
4-14-53 \\
11-17-53 \\
1-7-54 \\
10-15-54 \\
11-24-54 \\
7-22-55 \\
10-27-64 \\
12-15-64 \\
3-1-65\end{array}$ & $\begin{array}{l}.27 \\
3.67 \\
0 \\
1.47 \\
.3 .80 \\
.81 \\
0 \\
0 \\
2.20 \\
9.01 \\
31.0 \\
10.1\end{array}$ & $\begin{array}{r}5-18-65 \\
7-8-65 \\
10-11-65 \\
11-29-65 \\
1-11-66 \\
3-23-66 \\
6-1-66 \\
9-21-66 \\
2-28-67 \\
7-13-67 \\
8-23-67\end{array}$ & $\begin{array}{l}3.30 \\
0 \\
0 \\
0 \\
0 \\
0 \\
0 \\
23.0 \\
0 \\
0 \\
0\end{array}$ & $\begin{array}{r}10-4-67 \\
10-26-67 \\
12-6-67 \\
2-8-68 \\
4-10-68 \\
12-12-68 \\
3-19-69 \\
6-12-69 \\
11-6-69 \\
3-25-70 \\
5-18-70\end{array}$ & $\begin{array}{c}0 \\
0 \\
0 \\
17.0 \\
25.0 \\
2.0 \\
47.0 \\
4.4 \\
.94 \\
30.0 \\
20.0\end{array}$ & $\begin{array}{r}8-6-70 \\
11-18-70 \\
1-20-71 \\
4-7-71 \\
7-21-71 \\
10-19-71 \\
1-12-72 \\
4-14-72 \\
6-24-72 \\
10-11-72 \\
2-21-73 \\
5-15-73\end{array}$ & $\begin{array}{l}0 \\
0 \\
0 \\
0 \\
0 \\
1.1 \\
7.7 \\
0 \\
0 \\
0 \\
31 \\
26\end{array}$ \\
\hline
\end{tabular}

Wewoka Creek near Wetumka, Oklahoma, Miscellaneous site.

Lat. $35^{\circ} 11^{\prime} 45^{\prime \prime}$, long. $96^{\circ} 15^{\prime} 00^{\prime \prime}$, W line sec.3, T.8 N., R.10 E., at hridge on U.S. Highway 75, 2-1/2 miles south of Wetumka, Okia.

\begin{tabular}{|c|c|c|c|c|c|c|c|}
\hline Date & $\begin{array}{c}\text { Di scharge } \\
\left(\mathrm{ft}^{3} / \mathrm{s}\right)\end{array}$ & Date & $\begin{array}{c}\text { Discharge } \\
\left(\mathrm{ft}^{3} / \mathrm{s}\right)\end{array}$ & Date & $\begin{array}{c}\text { Discharge } \\
\left(\mathrm{ft}^{3} / \mathrm{s}\right)\end{array}$ & Date & $\begin{array}{c}\text { Discharge } \\
\left(\mathrm{ft}^{3} / \mathrm{s}\right)\end{array}$ \\
\hline $\begin{array}{r}9-27-51 \\
12-11-51 \\
1-15-52 \\
2-27-52 \\
6-3-52 \\
7-22-52 \\
9-2-52 \\
10-7-53 \\
11-18-53\end{array}$ & $\begin{array}{c}1.84 \\
7.52 \\
12.2 \\
87.1 \\
282.0 \\
1.48 \\
0 \\
16.6 \\
23.7\end{array}$ & $\begin{array}{r}12-29-53 \\
2-1-54 \\
3-9-54 \\
4-20-54 \\
6-1-54 \\
7-12-54 \\
8-10-54 \\
10-4-54 \\
12-16-54\end{array}$ & $\begin{array}{r}27.8 \\
28.2 \\
16.5 \\
12.7 \\
52.9 \\
.2 \\
1.53 \\
0 \\
5.68\end{array}$ & $\begin{array}{r}1-17-55 \\
3-2-55 \\
4-11-55 \\
5-31-55 \\
7-11-55 \\
8-16-55 \\
9-21-55 \\
10-26-55 \\
1-6-56\end{array}$ & $\begin{array}{l}16.8 \\
94.5 \\
13.7 \\
58.7 \\
3.74 \\
1.41 \\
.68 \\
1.66 \\
3.84\end{array}$ & $\begin{array}{r}2-13-56 \\
3-15-56 \\
4-24-56 \\
7-3-56 \\
8-27-56 \\
9-29-56 \\
11-14-56 \\
12-31-56 \\
10-5-57\end{array}$ & $\begin{array}{l}14.8 \\
5.13 \\
4.68 \\
5.08 \\
0 \\
0 \\
.88 \\
2.89 \\
3.29\end{array}$ \\
\hline
\end{tabular}

Wewoka Creek near Wewoka, Oklahoma, Miscellaneous site.

Lat. $35^{\circ} 12^{\prime} 32^{\prime \prime}$, Long. $96^{\circ} 31^{\prime} 07^{\prime \prime}$, SW 1/4 sec.11, T.8 N., R.7 E., at bridge on U.S. Highway 270,3 miles northwest of Wewoka, Okla.

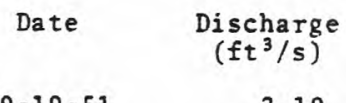

$9-19-51 \quad 2.19$

White Break Creek near Binger, Oklahoma, Miscellaneous site. Lat. $35^{\circ} 20^{\prime} 18^{\prime \prime}$, long. $98^{\circ} 15^{\prime} 42^{\prime \prime}$, NW $1 / 4$ sec.24, T.10 N., R.10 W., at head of Salyer Lake, 7 miles east of Binger, Ok1a.
Date
Discharge
$\left(\mathrm{ft}^{3} / \mathrm{s}\right)$
$9-19-56$
.02

Wildcat Creek near Herring, Oklahoma, Miscellaneous site.
Lat. $35^{\circ} 36^{\circ} 33^{\prime \prime}$, Iong. $99^{\circ} 32^{\prime} 48^{\prime \prime}$, Sk $1 / 4$ sec.23, T.13 $\mathrm{N}, \mathrm{R} .22 \mathrm{~K}$, belok dam, $1 / 4 \mathrm{mile}$ upstream from mouth:

Date Discharge

$2-12-57 \quad 0$ 
Wildhorse Creek near Hoover, Oklahoma, 07329700 , partial record site.

Lat. $34^{\circ} 32^{\prime} 29^{\prime \prime}$, long. $97^{\circ} 14^{\prime} 49^{\prime \prime}, W^{\prime} 1 / 2 \mathrm{sec} .19^{\prime}, \mathrm{T} .1 \mathrm{~N} ., \mathrm{R} .1 \mathrm{E}$, at bridge on State Highway $19 \mathrm{~A}, 1-1 / 2$ miles north of Hoover, Okla.

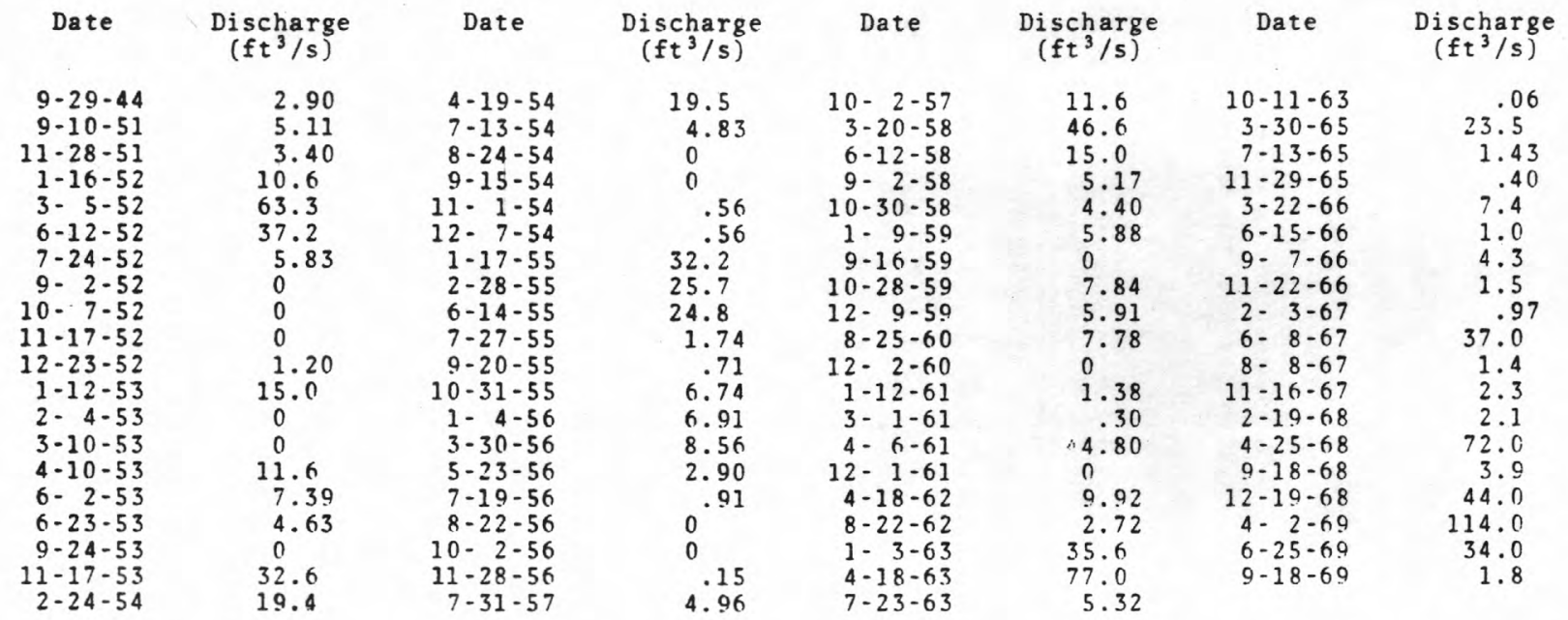

Wild Horse (Sand Creek) near Pond Creek, Oklahoma, 07150590 , partial record site.

Lat. $36^{\circ} 37^{\prime} 30^{\prime \prime}$, long. $9^{\circ} 48^{\prime} 00^{\prime \prime}$, on west line SW $1 / 4 \mathrm{SW} 1 / 4 \mathrm{sec} .24, \mathrm{~T} .25 \mathrm{~N} .$, R. 6 W., Grant County, at county road bridge on FAS road, 3 miles south of pond Creek and about $5 \mathrm{miles}$ upstream from mouth.

\begin{tabular}{|c|c|c|c|c|c|c|c|}
\hline Date & $\begin{array}{c}\text { Discharge } \\
\left(\mathrm{ft}^{3} / \mathrm{s}\right)\end{array}$ & Date & $\begin{array}{c}\text { Discharge } \\
\left(\mathrm{ft}^{3} / \mathrm{s}\right)\end{array}$ & Date & $\begin{array}{c}\text { Discharge } \\
\left(\mathrm{ft}^{3} / \mathrm{s}\right)\end{array}$ & Date & $\begin{array}{c}\text { Discharge } \\
\left(\mathrm{ft}^{3} / \mathrm{s}\right)\end{array}$ \\
\hline $\begin{array}{r}10-7-64 \\
1-18-65 \\
7-16-65 \\
12-6-65 \\
3-10-66 \\
6-30-66 \\
11-22-66\end{array}$ & $\begin{array}{l}0 \\
8.87 \\
3.79 \\
0 \\
.47 \\
0 \\
0\end{array}$ & $\begin{array}{r}1-31-67 \\
8-12-67 \\
1-29-68 \\
4-23-68 \\
7-18-68 \\
11-5-68 \\
2-14-69\end{array}$ & $\begin{array}{l}0 \\
0 \\
0 \\
0 \\
0 \\
0 \\
0\end{array}$ & $\begin{array}{r}4-16-69 \\
7-10-69 \\
10-23-69 \\
1-13-70 \\
4-8-70 \\
7-8-70 \\
10-20-70\end{array}$ & $\begin{array}{l}.44 \\
4.2^{2} \\
.10 \\
1.3^{3} \\
5.1 \\
0 \\
0\end{array}$ & $\begin{array}{r}3-23-71 \\
5-5-71 \\
8-17-71 \\
10-19-71 \\
3-9-72 \\
4-19-72 \\
8-\quad 2-72\end{array}$ & $\begin{array}{l}0 \\
0 \\
.20 \\
0^{.20} \\
.42 \\
.10 \\
0^{.10}\end{array}$ \\
\hline
\end{tabular}

Willow Creek near Swan Lake, Oklahoma, Miscellaneous site.

Lat. $35^{\circ} 13^{\prime} 00^{\prime \prime}$, long. $98^{\circ} 29^{\prime} 00^{\prime \prime}$, NE $1 / 4 \mathrm{sec} .32$, T.9 N., R.13 W., at county highway bridge, $2 \mathrm{miles}$ southeast of Swan Lake, Okla.

\begin{tabular}{|c|c|c|c|c|c|c|c|}
\hline Date & $\begin{array}{c}\text { Discharge } \\
\left(\mathrm{ft}^{3} / \mathrm{s}\right)\end{array}$ & Date & $\begin{array}{c}\text { Discharge } \\
\left(\mathrm{ft}^{3} / \mathrm{s}\right)\end{array}$ & Date & $\begin{array}{c}\text { Discharge } \\
\left(\mathrm{ft}^{3} / \mathrm{s}\right)\end{array}$ & Date & $\begin{array}{c}\text { Discharge } \\
(\mathrm{ft}: 3 / \mathrm{s})\end{array}$ \\
\hline $\begin{array}{r}9-15-52 \\
1-5-52\end{array}$ & $\begin{array}{l}0 \\
1.37\end{array}$ & $4-30-53$ & 2.13 & $6-22-53$ & 0 & $\begin{array}{l}9-8-53 \\
2-15-55\end{array}$ & $\begin{array}{l}0 \\
2.81\end{array}$ \\
\hline
\end{tabular}

Wolf Creek near Fort Supply, Oklahoma, Miscellaneous site.

Lat. $36^{\circ} 30^{\prime} 00^{\prime \prime}$, long. $99^{\circ} 34^{\prime} 12^{\prime \prime}$, SE $1 / 4$ sec.5, T.23 N., R.22 K., at head of Fort Supply Reservoir, 5-1/2 miles south of Fort Supply, Okla.

Date Discharge

$2-16-55 \quad .44$ 
LOW-FLOW MEASUREMENTS--COntinued.

Wolf Creek near Tangier, Oklahoma, Miscellaneous site.

Lat. $36^{\circ} 26^{\prime} 00^{\prime \prime}$, long. $99^{\circ} 35^{\prime} 30^{\prime \prime}$, SW $1 / 4$ sec. 29, T. 23 N., R. 22 W., at county bridge 3 miles northwest of Tangier, Okla.

\begin{tabular}{|c|c|c|c|c|c|c|c|}
\hline Date & $\begin{array}{c}\text { Discharge } \\
\left(f t^{3} / s\right)\end{array}$ & Date & $\begin{array}{c}\text { Discharge } \\
\left(\mathrm{ft}^{3} / \mathrm{s}\right)\end{array}$ & Date & $\begin{array}{c}\text { Discharge } \\
\left(f t^{3} / s\right)\end{array}$ & Date & $\begin{array}{c}\text { Discharge } \\
\left(\mathrm{ft}^{3} / \mathrm{s}\right)\end{array}$ \\
\hline $\begin{array}{l}10-4-41 \\
10-8-41 \\
10-15-41 \\
11-19-41 \\
11-28-41 \\
12-10-41 \\
12-15-41 \\
12-22-41 \\
12-30-41 \\
1-6-42 \\
1-13-42\end{array}$ & $\begin{array}{c}10.4 \\
60.7 \\
89.8 \\
109 . \\
114 . \\
830 . \\
130 . \\
93.3 \\
88.4 \\
42.4 \\
0\end{array}$ & $\begin{array}{l}1-19-42 \\
1-26-42 \\
2-2-42 \\
2-9-42 \\
2-18-42 \\
3-2-42 \\
3-9-42 \\
3-16-42 \\
3-23-42 \\
3-30-42\end{array}$ & $\begin{array}{r}112 . \\
82.1 \\
72.0 \\
64.3 \\
66.2 \\
86.6 \\
72.0 \\
69.3 \\
53.0 \\
63.2\end{array}$ & $\begin{array}{l}4-6-42 \\
4-13-42 \\
5-6-42 \\
5-19-42 \\
5-25-42 \\
6-2-42 \\
6-11-42 \\
6-18-42 \\
6-25-42 \\
6-30-42\end{array}$ & $\begin{array}{r}47.7 \\
71.2 \\
74.6 \\
56.7 \\
99.6 \\
28.0 \\
303.0 \\
85.0 \\
97.6 \\
78.1\end{array}$ & $\begin{array}{l}7-7-42 \\
7-14-42 \\
7-21-42 \\
7-29-42 \\
8-6-42 \\
8-13-42 \\
8-19-42 \\
8-31-42 \\
9-15-42 \\
9-28-42\end{array}$ & $\begin{array}{c}43.0 \\
14.7 \\
10.0 \\
2.46 \\
4.09 \\
479 . \\
67.9 \\
9.02 \\
7.32 \\
6.74\end{array}$ \\
\hline
\end{tabular}

Zane Cave Spring near Seneca, Oklahoma, Miscellaneous site.

Lat. $36^{\circ} 46^{\prime} 37^{\prime \prime}$, long. $99^{\circ} 39^{\prime} 32^{\prime \prime}, \mathrm{NE} 1 / 4 \mathrm{sec} .31$, T.27 N., R.25 E., at mouth of cave at Ozark Ranch, 4 miles southwest of West Seneca, Okla.

$\begin{array}{crrrrr}\text { Date } & \begin{array}{c}\text { Discharge } \\ \left(\mathrm{ft}^{3} / \mathrm{s}\right)\end{array} & \text { Date } & \begin{array}{c}\text { Discharge } \\ \left(\mathrm{ft}^{3} / \mathrm{s}\right)\end{array} & \text { Date } & \begin{array}{r}\text { Discharge } \\ \left(\mathrm{ft}^{3} / \mathrm{s}\right)\end{array} \\ 10-20-55 & 3.80 & 3-6-56 & 1.47 & 6-6-56 & 8.42\end{array}$


APPENDIX II

ALPHABETICAL AND STATION NUMBER INDICES

88 
Arkansas River at Ralston, Okla. Arkansas River at Tulsa, Okla. Arkansas River near Muskogee, Okla. Arkansas River near Sallisaw, Okla. Barnitz Creek near Arapaho; Okla.

Baron Fork at Eldon, Okla.

Bear Creek near Fallis, Okla.

Beaver Creek (Sand) Creek near Texhoma, Okla.07232400 Beaver Creek near Waurika, Okla.

Beaver River at Beaver, Okla.

Beaver River near Guymon, Okla.

Bellcow Creek at Chandler, Okla.

Bent Creek near Richmond, Okla.

Bent Creek near Selling, Okla.

Big Cabin Creek near Big Cabin, Okla.

Big Cabin Creek near Pyramid Corners, Okla.

Bird Creek at Avant, Okla.

Bird Creek near Sperry, Okla.

Bitter Creek near Chickasha, Okla.

Black Bear Creek at Pawnee, Okla.

Blue Beaver Creek near Cache, Okla.

Blue Creek near Connerville, Okla.

Blue Creek at Milburn, Okla.

Blue River near Blue, Okla.

Bluff Creek above Lake Hefner near Oklahoma City, OkLa.

Bois d'Arc Creek near Ponca City, Okla.

Buck Creek near Moyers, Okla.

Buffalo Creek near Lovedaie, Okla.

Byrd's Mill Spring near Fittstown, Okla,

Caddo Creek near Ardmore, Okla.

Canadian River at Bridgeport, Okla.

Canadian River at Calvin, Okla.

Canadian River near Newcastle, Okla.

Canadian River near Noble, Okla.

Canadian River near Whitefield, Okla.

Caney Creek near Copan, Okla.

Caney River near Bartlesville, Okla.

Caney River near Hulah, Okla.

Caney River near Ochelata, Okla.

Caney River near Ramona, Okla.

Captain Creek near Wellston, Okla.

Cedar Creek near Carnegie, Okla.

Chickasaw Creek near Stringtown, Okla.

Chikaskia R1ver near Blackwe11, Okla.

Cimarron River above Ute Creek near

Bolse City, Okla.

Cimarron River at Mannford, Okla.

Cimarron River at Oilton, Okla.

Cimarron River at Perkins, Okla.

Cimarron River near Buffalo, Okla.

Cimarron River near Guthrie, Okla.

Cimarron River near Kenton, Okla.

Cimarron River near Mocane, Okla.

Cimarron River near Waynoka, Okla.

Clear Boggy Creek near Caney, Okla.

Clear Boggy Creek near Frisco, Okla.

Clear Boggy Creek near Tupelo, Okla.

Clear Creek near Elmwood, Okla.

Clear Creek near May, Okla.

Cobb Creek near Fort Cobb, Okla.

Cold Springs Creek near Castaneda, Okla.

Coldwater Creek near Hardesty, Okla.

Commission Creek near Grand, Okla.

Cottonwood Creek near Guthrie, Okla.

Cottonwood Creek near Navina, Okla.

Cottonwood Creek near Seward, Okla.

Counc11 Creek near St1llwater, Okla.

Cow Creek near Waurika, Okla.

Deep Fork near Beggs, Okla.

Deep Fork near Dewar, Okla

Deep Fork near Welty, Okla.
07152500

07164500

07194500

07246500

07324500

07197000

1

07313500

07234000

07232500

07242500

07237800

07237800

07191000

07190600

07176500

07177500

07153000

07311200

07332400

07332500

07159500

07152250

07335900

07157960

07334200

07330500

07228500

07231500

07229000

07229100

07245000

07174000

07174500

07173000

07174700

07175500

8

07333500

07152000

07155000

07164000

07163500

07161000

07157950

07160000

07154500

07157000

07158000

07335000

12

07334400

07234100

07234300

07326000

07155200

07233000

07228150

07159800

14

07159750

07163000

07313600

07243500

07244000

15
Deep Red Run near Randlett, Okla.

Deer Creek near Edmond, Okla.

Deer Creek at Hydro, Okla.

Deer Creek near Tonkawa, Okla.

Delaware Creek near Wapunucka, Okla.

Dirty Creek near Warner, Okla.

Dry Creek near Kendrick, Okla.

Duck Pond Creek near Clear Lake, Okla.

Eagle Chief Creek near Aline, Okla.

Eagle Chief Creek near Carmen, Okla.

$\begin{array}{ll}\text { Eagle Chief Creek near Cleo, Okla. } & 20 \\ \text { East Branch Sandstone Creek near E1k C1ty, Okla. } & 07322500\end{array}$

East Cache Creek near Walters, Okla. 07311000

Elk Creek near Hobart, Okla.

07304500

E1m Fork of North Fork Red RIver near Car1, Okla. 07303400

Elm Fork of North Fork Red River near Mangum, Okla.

Finn Creek near Story, Okla.

Five Mile Creek near Eakley, Okla.

Flint Creek near Kansas, Okla.

Fourche Maline near Red Oak, Okla.

Gaines Creek near Krebs, Okla.

Glover Creek near Glover, Okla.

Goff Creek near Guymon, Okla.

Goff Creek near Haugh, Okla.

Gypsum Creek near Olustee, Okla.

Hickory Creek near Marietta, Okla.

Hominy Creek near Skiatook, Okla.

Horse Creek at Afton, Okla.

House Creek near Terleton, Okla.

Hoyle Creek near Amco, Okla.

Illinois River near Gore, Okla.

Illinois River near Tahlequah, Okla.

07311500

07150900
07334440

07198500

07243000

07234130

07158100

Ilinois River near Watts, Qkla.

Indian Creek near Woodward, Okla.

Island Bayou near Albany, Okla.

Kiamichi River near Belzoni, Okla.

Kiamichi River near Big Cadar, Okla

Kingfisher Creek near Kingfisher, Okla.

Klowa Creek near Slapout, Okla.

Lake Creek near Eakly, Okla.

Leader Creek at Tupelo, Okla.

Lee Creek near Short, Okla.

Little Beaver Creek near Duncan, Okla.

Little Caney River below Cotton Creek near

Copan, Okla.

Little Deep Fork near Edna, Okla.

07303500

07328300

21

07196000

07247500

07232000

07337900

07232590

07232580

07299780

07316070

07177000

07189700

24

25

07198000

07196500

07195500

26

07332250

07336500

07335700

07159200

07234200

07325850

07334420

07249800

07313000

07174200

30

Little Lee Creek near Short, Okla.

Little Medicine Bluff Creek near Lawton, 0kla 0731000

Little River near Idabel, Okla.

Little River below Lake Thunderbird

near Norman, Okla.

07338000

Little River near Tecumseh, Okla.

Little River bli Lukfata Creek or Idable, Okla. 07338500

Little River near Sasakwa, Okla.

Little River near Wright City, Okla.

Little Washita R1ver at Ninnekah, Okla.

Little Washita River near Ninnekah, Okla.

Lost Creek at Seneca, Mo.

Main Creek near Waynoka, Okla.

McGee Creek near Stringtown, Okla.

Medicine Bluff Creek near Lawton, Okla.

Mi11 Creek near Mill Creek, Okla.

Mountain Fork near Eagletown, Okla.

Mud Creek near Courtney, Okla.

Muddy Boggy Creek near Farris, Okla.

Muddy Boggy Creek near Parker, Okla.

Neosho River below Fort Gibson Lake

near Fort Gibson, Okla.

Neosho RIver near Chouteau, Okla.

Neosho River near Commerce, Okla.

Neosho River near Grove, Okla.
07338500
07231000

07337500

07327500

07327490

07188500

07158010

07333800

07310500

07331200

07339000

07315700

07334000

07332700

07193500

07191500

07185000

07189500 
Neosho Rlver near Langley, Okla. Neosho RIver near Wagoner, Okla.

North Boggy Creek near Atoka, Okla.

North Canadian RIver at Canton, Okla. 07239000

North Canadian River at Woodward, Okla. 07237500

North Canadian River near E1 Reno, Okla. 07239500

North Canadian River near Harrah, Okla. 07241500

North Canadian River near Oklahoma C1ty,0kla.07241500

North Canadian River near Selling, Okla, 07238000

North Canadian River near Wetumka, Okla. 07242000

North Fork Red RIver below Altus Dam

near Lugert, Okla.

North Fork Red River near Carter, Okla.

North Fork Red River near Granite, Okla.

North Fork Red River near Headrick, Okla.

North Fork Red River near Texola, Okla.

Otter Creek at Mountain Park, Okla.

Palo Duro Creek near Range, Okla.

Pennington Creek near Reagan, Okla.

Persimmon Creek (North) near Sharon, Okla.

Persimmon Creek (South) near Sharon, Okla.

Persimmon Creek near Mutual, Okla.

Polecat Creek below Heyburn Lake

near Heyburn, Okla.

Pond Creek near Lamont, Okla.

Pony Creek near Oprima, Okla.

Poteau River at Poteau, Okla.

Poteau River near Wister, Okla.

Preacher Creek near Dover, Okla.

Pryor Creek near Pryor, Okla.

$\begin{array}{ll}\text { Quapaw Creek near Meeker, Okla. } & 42 \\ \text { Rainy Mountain Creek near Mountain View, Okla.07325300 }\end{array}$

Red River at Arthur City, Tex.

Red River near Colbert, Okla. 07332000

Red River at Denison Dam near Denison, Tex. 07331600

Red River near Terral, Okla.

Red Rock Creek near Red Rock, Okla. 43

Rock Creek at Dougherty, Okla. 07329900

Roundup Creek near Woodward, Okla. 45

Rush Creek at Purdy, Okla.

Salina Creek near Salina, Okla.

Sallisaw Creek near Sallisaw, 0kla. 07245500

Salt Creek near Dewright, Okla. 07230800

Salt Creek near Okeene, Okla. 07158400

Salt Creek near Pearson, Okla.

49

Salt Creek near Shidler, Okla. 07152400

Salt Fork Arkansas River at Tonkawa, Okla. 07151000

Salt Fork Arkansas River near Alva, Okla. 07148400

Salt Fork Arkansas River near Cherokee, Okla.07149500

Salt Fork Arkansas River near Jet, Okla. 07150500

Salt Fork Arkansas River ir Winchester, Okla.07148350

Salt Fork Arkansas River at Mangum, Okla. 07300500

Sand Creek at. Okesa, Okla.

Sandstone Creek near Berlin, Okla.

Sandstone Creek near Cheyenne, Okla.

Sandy Creek near Bryan, Okla.

Skeleton Creek near Lovell, Okla.

Snake Creek near Bixby, Okla.

Spavinaw Creek near Sycamore, Okla.

Spring Creek near Ada, Okla.

Spring River near Quapaw, Okla.

Starvation Creek near Prentiss, Okla.

Stinking Creek near Carnegie, Okla.

Sugar Creek near Gracemont, Okla.

Suttle Creek near Davidson, ORla.

Sweetwater Creek near Texola, Okla.

Tenmile Creek near Miller, Okla.

Tepee Creek near Eva, Okla.

Turkey Creek near Dover, Okla.

Turkey Creek near Drummond, Okla.

Turkey Creek at Olustee, Okla.

07174600

07319500

07323000

07149700

07160500

07165550

07191220

51

07188000

07301452

07325400

07327000

07308310

52

07336000

07232450

54

07159000

07301100
Turkey Creek near Sayre, Okla.

Verdigris River near Claremore, Okla.

Verdigris River near Inola, Okla.

Verdigris River near Lenapah, Okla.

Verdigris Rfver near Oologah, Okla.

Verdigris River near Sageeyah, Okla.

Walnut Bayou near Burneyville, Okla.

Walnut Creek near Purcell, Okla.

Washita River at Anadarko, Okla.

Washita River at Carnegie, Okla.

Washita River near Cheyenne, Okla.

Washita River near Clinton, Okla.

Washita River near Durwood, Okla.

Washita River near Foss, Okla.

Washita River near Hammon, Okla.

Washita River near Pauls Velley, Okla.

Washita River near Tabler, Okla.

West Bitter Creek near Tabler, Okla.

West Cache Creek near Cookietown, Okla.

West Otter Creek at Snyder Lake

near Mountain Park, Okla.

Wewoka Creek near Wetumka, Okla.

Wildhorse Creek near Hoover, Okla.

Wildhorse (Sand) Creek near Pond Creek, Okla.

Winter Creek near Alex, Okla.

Wolf Creek near Fargo, Okla.

Wolf Creek near Fort Supply, Okla.

Wolf Creek near Shattuck, Okla.
07301460

07176000

07178600

07171000

07171400

07171500

07315900

07229300

07326500

07325500

07316500

07325000

07331000

07324400

07324200

07328500

07328000

55

073112400

07305500

56

07329700

07150590

07328070

07236000

07237000

07235500 
07148350 Salt Fork Arkansas near W1nchester, Okla.

07148400 Salt Fork Arkansas near Alva, Okla.

07149500 Salt Fork Arkansas near Cherokee, Okla.

07149700 Sandy Creek near Bryon, Okla.

07150500 Salt Fork Arkansas near Jet, Okla.

07150590 W1ldhorse (Sand) Creek near Pond Creek, Okla.

07150900 Deer Creek near Tonkawa, Okla.

07151000 Salt Fork Arkansas at Tonkawa, Okla. (unregulated)

07151000 Salt Fork Arkansas at Tonkawa, Okla. (regulated)

07152000 Chikaskia River near Blackwell, Okla.

07152250 Bois D'Arc Creek near Ponca City, Okla.

07152400 Salt Creek near Shidler, Okla.

07152500 Arkansas River at Ralston, Okla. (unregulated)

07152500 Arkansas River at Ralston, Okla. (regulated)

07153000 Black Bear Creek at Pawnee, Okla.

07154500 Cimarron River near Kenton, Okla.

07155000 Cimarron River above Ute Creek near Boise City, Okla.

07155200 Cold Springs Creek near Castanada, Okla.

07157000 Cimarron River near Mocane, Okla.

07157950 Cimarron RIver near Buffalo, Okla.

07157960 Buffalo Creek near Lovedale, Okla.

07158000 Cimarron River near Waynoka, Okla.

07158010 Main Creek near Waynoka, Okla.

07158100 Eagle Chief Creek near Aline, Okla.

07158400 Salt Creek near Okeene, Okla.

07158500 Preacher Creek near Dover, Okla.

07159000 Turkey Creek near Drummond, Okla.

07159200 Kingfisher Creek near Kingfisher, Okla.

07159500 Bluff Creek above Lake Hefner near Oklahoma City, Okla.

07159750 Cottonwood Creek near Seward, Okla.

07159800 Cottonwood Creek near Guthrie, Okla.

07160000 Cimarron River near Guthrie, Okla.

07160500 Skeleton Creek near Lovell, Okla.

07161000 Cimarron River at Perkins, Okla.

07163000 Council Creek near Stillwater, Okla.

07163500 Cimarron River near Oilton, Okla.

07164000 Cimarron River at Mannford, Okla.

07164500 Arkansas River at Tulsa, Okla. (unregulated)

07164500 Arkansas River at Tulsa, Okla. (regulated)

07165500 Polecat Creek below Heyburn Lake near Heyburn, Okla. (unregulated)

07165500 Polecat Creek below Heyburn Lake near Heyburn, Okla. (regulated)

07165550 Snake Creek near Bixby, Okla.

07171000 Verdigris River near Lenapah, Okla. (unregulated)

07171000 Verdigris River near Lenapah, Okla. (regulated)

07171400 Verdigris River near Oologah, Okla.

07171500 Verdigris River near Sageeyah, Okla.

07173000 Caney River near Hulah, Okla. (unregulated)

07173000 Caney River near Hulah, Okla. (regulated)

07174000 Caney Creek near Copan, Okla.

07174200 Little Caney River below Cotton Creek uear Copan, 0kla.

07174500 Caney River at Bartlesville, Okla.

07174600 Sand Creek near Okesa, Okla.

07174700 Caney River near Ochelata, Okla.

07175500 Caney R1ver near Ramona, Okla. (unregulated)

07175500 Caney River near Ramona, Okla. (regulated)

07176000 Verdigris River near Claremore, Okla. (unregulated)

07176000 Verdigris River near Claremore, Okla. (regulated)

07176500 Bird Creek near Avant, Okla.

07177000 Hominy Creek near Skiatook, Okla.
07177500 Bird Creek near Sperry, Okla.

07178600 Verdigris River near Inola, Okla.

07185000 Neosho River near Commerce, Okla. (unregulated)

07185000 Neosho R1ver near Commerce, Okla. (regulated)

07188000 Spring River near Quapaw, Okla.

07188500 Lost Creek at Seneca, Mo.

07189500 Neosho River near Grove, Okla.

07189700 Horse Creek at Afton, Okla.

07190500 Neosho River near Langley, Okla.

07190600 Big Cabin Creek near Pyramid Corners, Okla.

07191000 Big Cabin Creek near Big Cabin, Okla.

07191220 Spavinaw Creek near Sycamore, Okla.

07191500 Neosho River near Chouteau, Okla.

07192000 Pryor Creek near Pryor, Okla.

07192500 Neosho River near Wagoner, Okla.

07193500 Neosho RIver below Fort Gibson Lake near Fort Gibson, Okla.

07194500 Arkansas River near Muskogee, Okla. (unregulated)

07194500 Arkansas River near Muskogee, Okla. (regulated)

07195500 Illinois River near Watts, Okla.

07196000 Fling Creek near Kansas, Okla.

07196500 Illinois River near Tahlequah, Okla.

07197000 Baron Fork at Eldon, Okla.

07198000 Illinois River near Gore, Okla, (unregulated)

07198000 Illinois River near Gore, Okla. (regulated)

07198500 D1rty Creek near Warner, Okla.

07228150 Commission Creek near Grand, Okla.

07228220 Turkey Creek near Camargo, Okla.

07228400 Deer Creek at Hydro, Okla.

07228500 Canadian River at Bridgeport, Okla. (unregulated)

07228500 Canadian River at Bridgeport, Okla. (regulated)

07229000 Canadian River near Newcastle, Okla.

07229100 Canadian River near Noble, Okla.

07229300 Walnut Creek at Purcel1, Okla.

07230000 Litrle River below Lake Thunderbird near Norman, Okla. (unregulated)

07230000 Litcle River below Lake Thunderbird near Norman, Okla. (regulated)

07230500 Litrle River near Tecumseh, Okla, (unregulated)

07230500 Little River near Tecumseh, Okla. (regulared)

07230800 Salt Creek near Dewright, Okla.

07231000 Little River near Sasakwa, Okla. (unregulated)

07231000 Little River near Sasakwa, Okla. (regulated)

07231500 Canadian River near Calvin, Okla. (unregulated)

07231500 Canadian River at Calvin,-Okla. (regulated)

07232000 Gaines Creek near Krebs, Okla.

07232400 Beaver (Sand) Creek near Texhoma, Okla.

07232450 Tepee Creek near Eva, Okla.

07232500 Beaver River near Guymon, Okla.

07232580 Goff Creek near Hough, Okla.

07232590 Goff Creek near Guymon, Okla.

07232620 Pony Creek near Optima, Okla.

07233000 Coldwater Creek near Hardesty, Okla.

07233700 Palo Duro Creek near Range, Okla.

07234000 Beaver River at Beaver, Okla.

07234100 Clear Creek near Elmwood, Okla.

07234130 Duck Pond Creek near Clear Lake, Okla.

07234200 Klowa Creek near Slapout, Okla.

07234300 Clear Creek near May, Okla.

07234500 North Canadian River near Fort Supply, Okla.

07235500 Wolf Creek near Shattuck, Okla.

07236000 Wolf Creek near Fargo, Okla.

07237000 Wolf Creek near Fort Supply, Okla.

07237500 North Canadian River at Woodward, Okla. (unregulated)

07237500 North Canadian River at Woodward, Okla. (regulated)

07237700 Persimmon Creek near Mutual, Okla. 
07237800 Bent Creek near Richmond, Okla.

07237800 Bent Creek near Selling, Okla.

07238000 North Canadian River near Selling, Okla.

07239000 North Canadian River at Canton, Okla. (unregulated)

07239000 North Canadian River at Canton, Okla. (regulated)

07239500 North Canadian River near E1 Reno, Okla. (unregulated)

07239500 North Canadian River near El Reno, Okla. (regulated)

07241500 North Canadian River near Oklahoma City, Okla.

07241500 North Canadian River near Harrah, Okla.

07242000 North Canadian River near Wetumka, Okla.

07242500 Bellcow Creek at Chandler, Okla.

07243000 Dry Creek near Kendrick, Okla.

07243500 Deep Fork near Beggs, Okla.

07244000 Deep Fork near Dewar, Okla.

07245000 Canadian River near Whitefield, Okla. (unregulated)

07245000 Canadian River near Whitefield, Okla. (regulated)

07245500 Sallisaw Creek near Sallisaw, Okla.

07246500 Arkansas River near Sallisaw, Okla. (unregulated)

07246500 Arkansas River near Sallisaw, Okla. (regulated)

07247500 Fourche Maline near Red Oak, Okla.

07248500 Poteau River near Wister, Okla. (unregulated)

07248500 Poteau River near Wister, Okla. (regulated)

07249000 Poteau River near Poteau, Okla.

07249800 Lee Creek near Short, Okla.

07249900 Little Lee Creek near Short, Okla.

07250000 Lee Creek near Van Buren, Ark.

07300500 Salt Fork Red River at Mangum, Okla.

07301100 Turkey Creek at Olustee, Okla.

07301452 Starvation Creek near Prentiss, Okla.

07301460 Turkey Creek near Sayre, Okla.

07301500 North Fork Red River near Carter, Okla.

07302000 North Fork Red River near Granite, Okla.

07303000 North Fork Red River below Altus Dam near Lugert, Okla.

07303400 Elm Fork of North Fork Red River near Car1, Okla.

07303500 EIm Fork of North Fork Red River near Mangum, Okla.

07304500 Elk Creek near Hobart, Okla.

07305000 North Fork Red River near Headrick, Okla. (unregulated)

07305000 North Fork Red River near Headrick, Okla. (regulated)

07305500 West Otter Creek at Snyder Lake near Mountain Park, Okla.

07306500 Otter Creek at Mountain Park, Okla.

07308310 Suttle Creek near Davidson, Okla.

07310000 Little Medicine Bluff Creek near Lawton, Okla.

07310500 Medicine Bluff Creek near Lawton, Okla.

07311000 East Cache Creek near Walters, Okla.

07311200 Blue Beaver Creek near Cache, Okla.

07311240 West Cache Creek near Cookfetown, Okla.

07311500 Deep Red Run near Randlett, Okla.

07313000 Litrle Beaver Creek near Duncan, Okla.

07313500 Beaver Creek near Waurika, Okla.

07313600 Cow Creek near Waurika, Okla.

07315500 Red River near Terral, Okla.

07315700 Mud Creek near Courtney, Okla.

07315900 Walnut Bayou near Burneyville, Okla.
07316070 Hickory Creek near Marietta, Okla.

07316500 Washita River near Cheyenne, Okla. (unregulated)

07316500 Wash1ta River near Cheyenne, Okla. (regulated)

07319500 Sandstone Creek near Berl1n, Okla.

07322500 East Branch Sandstone Creek near E1k C1ty, Okla.

07323000 Sandstone Creek near Cheyenne, Okla.

07324200 Washita River near Hammon, Okla.

07324400 Washita River near Foss, Okla.

07324500 Barnitz Creek near Arapaho, Okla.

07325000 Washita River near Clinton, Okla. (unregulated)

07325000 Washita River near Clinton, Okla. (regulated)

07325300 Rainy Mountain Creek near Mountain V1ew, Okla.

07325400 Stinking Creek near Carnegie, Okla.

07325500 Washita River at Carnegie, Okla. (unregulated)

07325500 Washita River at Carnegle, Okla. (regulated)

07325800 Cobb Creek near Eakly, Okla.

07325850 Lake Creek near Eakly, Okla.

07326000 Cobb Creek near Fort Cobb, Okla. (unregulated)

07326000 Cobb Creek near Fort Cobb, Okla. (regulated)

07326500 Washita River at Anadarko, Okla. (unregulated)

07326500 Washita River at Anadarko, Okla. (regulated)

07327000 Sugar Creek near Gracemont, Okla. (unregulated)

07327000 Sugar Creek near Gracement, Okla. (regulated)

07327490 Little Washita River near Ninnekah, Okla.

07327500 Little Washita River at Ninnekah, Okla.

07328000 Washita River near Tabler, Okla.

07328070 Winter Creek near Alex, Okla.

07328300 Finn Creek near Story, Okla.

07328500 Washita River near Pauls Valley, Okla.

07329000 Rush Creek at Purdy, Okla.

07329500 Rush Creek near Maysville, Okla. (unregulated)

07329500 Rush Creek near Maysville, Okla. (regulated)

07329700 Wildhorse Creek near Hoover, Okla.

07329900 Rock Creek at Dougherty, Okla.

07330500 Caddo Creek near Ardmore, Okla.

07331000 Washita River near Durwood, Okla.

07331200 Mill Creek near Mill Creek, Okla.

07331300 Pennington Creek near Reagon, Okla.

07331600 Red River at Denison Dam near Denison, Tex.

07332000 Red River near Colbert, Okla. (unregulated)

07332000 Red River near Colbert, Okla. (regulated)

07332250 Island Bayou near Albany, Okla.

07332400 Blue Creek at Mtlburn, Okla.

07332500 Blue River near Blue, Okla.

07332700 Muddy Boggy Creek near Parker, Okla.

07333500 Chickasaw Creek near Stringtown, Okla.

07333800 McGee Creek near Stringtown, Ok1a.

07334000 Muddy Boggy Creek near Ferris, Okla.

07334200 Byrds Mill Spring near Fittstown, Okla.

07334400 Clear Boggy Creek near Tupelo, Okla.

07334420 Leader Creek at Tupelo, Okla.

07334440 Delaware Creek near Wapunucka, Okla.

07335000 Clear Boggy Creek near Caney, Okla.

07335500 Red River at Arthur C1ty, Tex. ᄀ-

07335700 Riamichi River near Big Cedar, Okla.

07335900 Buck Creek near Moyers, Okla.

07336000 Tenmile Creek near Miller, Okla.

07336500 Kiamich1 River near Belzoni, Okla.

07337500 Little River near Wright City, Okla. (unregulated

07337500 Little River near Wright C1ty, Okla. (regulated)

07337900 Glover Creek near Glover, Okla.

07338000 Little River near Idabel, Okla.

07338500 Little River below Lukfata Creek near Idabel, Okla. (unregulated)

07338500 Little River below Lukfata Creek near Idabel, Okla. (regulated)

07339000 Mountain Fork near Eagleton, Okla. (unregulated) 
Bear Creek near Fall1sa, Okla.

Bitter Creek near Chickasha, Okla.

Blue Creek near Connerville, Okla.

Captain Creek near Wellston, Okla.

Cedar Creek near Carnegie, Okla.

Clear Boggy near Frisco, Okla.

Cottonwood Creek near Navina, Okla.

Deep Fork near Welty, Okla

Deer Creek near Edmond, Okla.

Eagle Chief Creek near Carmen, Okla.

Eagle Chief Creek near Cleo, Okla.

Five Mile Creek near Eakley, Okla.

House Creek near Terleton, Okla.

Hoyle Creek near Ames, Okla.

Indian Creek near Woodward, Okla.

(sec. 29, T22N, R2OW)

Indian Creek near Woodward, Okla.

(sec. $30, \mathrm{~T} 22, \mathrm{R} 19$ )

Indian Creek near Woodward, Okla.

(sec. 21, T22, R19)

Little Deep Fork near Edna, Okla.

North Boggy Creek near Atoka, Okla.

North Fork Red River near Texola, Okla.

Persimmon Creek (North) near Sharon, Okla.

Persimmon Creek (South) near Sharon, Okla.

Pond Creek near Lamont, Okla.

Quapaw Creek near Meeker, Okla.

Red Rock Creek near Red Rock, Okla.

Roundup Creek near Woodward, Okla.

Salina Creek near Salina, Okla.

Salt Creek near Pearson, Okla.

Spring Creek near Ada, Okla.

Sweetwater Creek near Texola, Okla.

Turkey Creek near Dover, Okla.

West Bitter Creek near Tabler, Okla.

Wewoka Creek near Wetumka, Okla. 



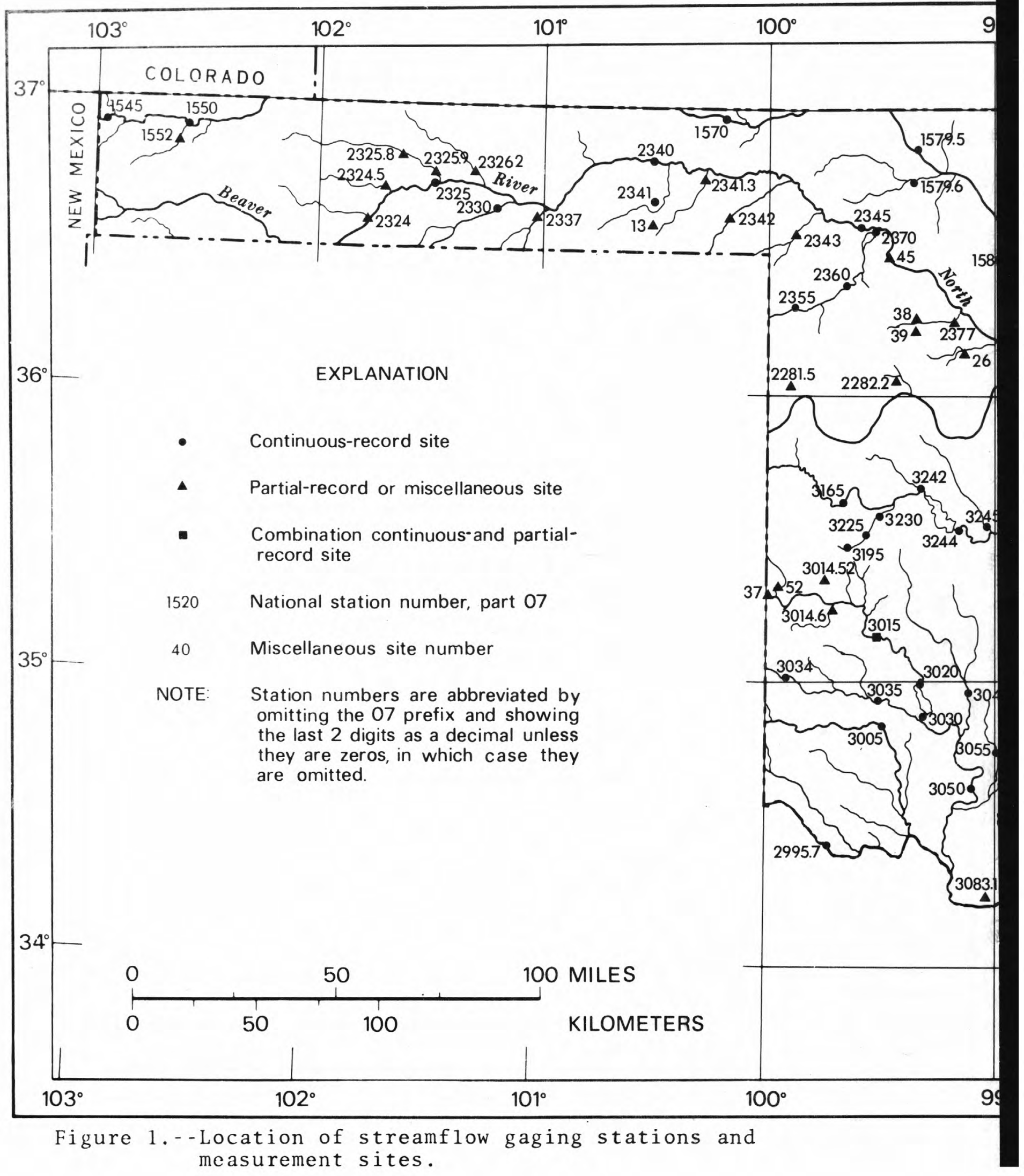


